\title{
HIGH SENSITIVITY FIELD ASYMMETRIC ION MOBILITY SPECTROMETER
}

\author{
THĖSE NO 7128 (2016) \\ PRÉSENTÉE LE 19 AOÛT 2016 \\ À LA FACULTÉ DES SCIENCES ET TECHNIQUES DE L'INGÉNIEUR \\ LABORATOIRE DE MICROSYSTĖMES 1 \\ PROGRAMME DOCTORAL EN MICROSYSTĖMES ET MICROÉLECTRONIQUE \\ ÉCOLE POLYTECHNIQUE FÉDÉRALE DE LAUSANNE \\ POUR L'OBTENTION DU GRADE DE DOCTEUR ÈS SCIENCES
}

PAR

Mario Andrés CHAVARRÍA VARÓN

acceptée sur proposition du jury:

Prof. S. Lacour, présidente du jury

Prof. J. Brugger, Dr G. Boero, directeurs de thèse

Dr W. Vautz, rapporteur

Dr M. Grössl, rapporteur

Prof. Ph. Renaud, rapporteur

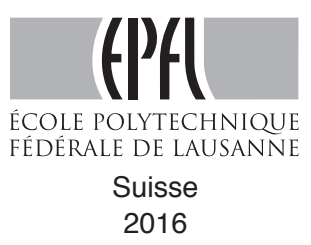



A mis padres... 



\section{Abstract}

Field Asymmetric Ion Mobility Spectrometry (FAIMS) is a very promising technique for chemical detection. FAIMS systems are portable, highly sensitive, relatively inexpensive and can work at atmospheric conditions. These attributes make it a very desirable technique to replace current bulky and expensive detection systems, such as Mass Spectrometry devices, especially for on-site experiments. Therefore, this work focuses on improving the FAIMS limits of detection (LoD) in order to expand the application fields for FAIMS technology. With this aim, the different factors affecting the FAIMS sensitivity and resolution were analyzed by theoretical and experimental studies.

An ion mobility simulation software was developed based on the physical models for FAIMS proposed in literature. This software is able to simulate the ion trajectories inside the ion filter and the expected ion spectra, making it an important tool for theoretically determining the effect of different parameters in FAIMS measurements. It also helps the user to predict and understand the experimental results.

The possibility of improving the fabrication process was evaluated by implementing two different techniques: PCB manufacturing and screen printing. The PCB plates were fabricated on FR4 substrates with copper electrodes while the screen printed plates used alumina substrates and silver-palladium electrodes. The prototypes developed with PCB techniques showed a very similar behavior to the standard alumina systems. Therefore, the PCB fabrication approach is a good option to reduce the fabrication complexity and costs of the FAIMS devices.

Two atmospheric pressure ionization sources, corona discharge and UV, were tested for possible use in the FAIMS setup. Both sources showed similar ionization efficiency levels. Between the two sources, the UV is a simpler system that requires very low optimization and has very stable ionization rates. Consequently, it was selected as the ionization method for this work. All the required electronics for the ions filtering and detection were also developed. The designed separation voltage circuit, based on the power nMOS inverter configuration, is able to produce rectangular waveforms of up to $1000 \mathrm{~V}_{\mathrm{PP}}$ in the $\mathrm{MHz}$ range. The compensation voltage (CV) circuit can scan the ion spectra by generating a voltage ramp from -20 to $20 \mathrm{~V}$. The fabricated low current amplifier has a gain of $10^{11}$ with a noise of about $4 \mathrm{fA} / \sqrt{\mathrm{Hz}}$ limited by the thermal noise of the feedback resistor. The experimental parameters can be controlled and monitored by a custom user interface software developed in LabVIEW ${ }^{\circledR}$. This software also digitalizes, displays and save the measured data.

Finally, several measurements were performed to determine the influence of the different 
experimental parameters in the FAIMS sensitivity and resolution. In order to have a better understanding of the phenomena occurring within the FAIMS, the parameters' effects on each of the FAIMS section were studied separately. Also, for the first time, the effects of a synchronous modulation technique (lock-in) in the FAIMS ion current detection were analyzed. Based on the results of these studies, the LoD of the FAIMS system was significantly improved. The extrapolated acetone LoD levels, from the experimental signals' amplitude and base line noise, of the developed device are $900 \mathrm{ppt}$ and $40 \mathrm{ppt}$ at carrier gas flows of 3.05 and $9.5 \mathrm{l} / \mathrm{min}$ respectively. These LoD levels are similar or slightly better than the state of the art levels for standalone FAIMS published in literature.

Key words: High Field Ion Mobility Spectrometry (FAIMS), Ion Mobility, chemical detection, Ion filtering, Ion detection, Limit of Detection (LoD), UV photoionization, Low noise amplifier, Lock-in detection. 


\section{Riassunto}

La spettrometria di mobilità ionica per campi asimmetrici (FAIMS) è una tecnica analitica molto promettente nel campo dell'identificazione di sostanze chimiche. I sistemi FAIMS vantano portabilità, elevata sensibilità, basso costo e possibilità di utilizzo in condizioni ambiente. Tali caratteristiche rendono questa tecnica un'ottima candidata a sostituire le attuali strumentazioni disponibili sul mercato, in particolar modo per misure in-situ. I comuni sistemi di spettrometria di massa, infatti, risultano decisamente più costosi e ingombranti. L'oggetto di questo lavoro di tesi è il miglioramento del limite di rivelazione (LoD) dei sistemi FAIMS, al fine di espandere questa tecnologia. Per raggiungere tale scopo sono stati analizzati i diversi fattori che influenzano la sensibilità e la risoluzione del sistema FAIMS, conducendo uno studio sia analitico che sperimentale.

Nel corso di questo lavoro è stato sviluppato un sofware adibito alla simulazione della mobilità ionica, basato sui modelli fisici dei FAIMS presenti in letteratura. Tale software rappresenta uno strumento fondamentale per la determinazione degli effetti dei diversi parametri sulle misure FAIMS, in quanto permette la simulazione dello spettro ionico atteso e delle traiettorie degli ioni all'interno del filtro ionico. Queste informazioni aiutano a predirre e comprendere i risultati sperimentali.

Due diverse tecniche sono state implementate al fine di migliorare il processo di fabbricazione: la realizzazione di una PCB e la serigrafia. La PCB è stata fabbricata su un substrato FR4 con elettrodi di rame, mentre la serigrafia è stata realizzata su placche di allumina con elettrodi in argento-palladio. I prototipi PCB hanno mostrato un comportamento molto simile a quello dei piu' comuni e complessi sistemi in allumina. Di conseguenza questo lavoro è stato basato sulla realizzazione di una $\mathrm{PCB}$, consentendo una riduzione dei costi e della complessità della fabbricazione rispetto ai sistemi FAIMS in allumina.

Sono state testate due sorgenti di ionizzazione: la scarica effetto corona e l'ultravioletto (UV). Nonostante i due sistemi abbiano dimostrato simili valori di efficienza di ionizzazione, in questo lavoro la sorgente UV è stata preferita alla scarica effetto corona. Il motivo di tale scelta risiede nel fatto che la sorgente UV è un sistema piu' semplice che non necessita di particolare ottimizzazione, ed ha valori di ionizzazione molto stabili.

Sono state inoltre sviluppate tutte le varie componenti elettroniche necessarie per il filtraggio e la rivelazione degli ioni. Il circuito di tensione di separazione sviluppato in questa tesi, basato sulla configurazione inverter per transistor nMOS di potenza, produce una forma d'onda rettangolare fino a $1000 \mathrm{~V}_{\mathrm{PP}}$ nel range dei $\mathrm{MHz}$. Il circuito di compensazione di tensione (CV) è in grado di analizzare lo spettro ionico generando una rampa di tensione da -20 a 
$20 \mathrm{~V}$. L'amplificatore di bassa corrente che è stato fabbricato ha un guadagno di $10^{11} \mathrm{ed}$ un rumore di circa $4 \mathrm{fA} / \sqrt{\mathrm{Hz}}$, limitato dal rumore termico della resistenza di feedback. E' possibile controllare e monitorare i parametri sperimentali tramite un software interfaccia utente sviluppato in LabVIEW. Tale software, inoltre, digitalizza, mostra e salva i dati misurati. Sono state effettuate molte misure al fine di determinare l'influenza dei diversi parametri sperimentali sulla sensibilità e sulla risoluzione del FAIMS. Per avere una chiara comprensione dei fenomeni fisici in atto è stato studiato l'effetto dei singoli parametri su ognuna delle sezioni del FAIMS. Inoltre, sono stati analizzati per la prima volta gli effetti della tecnica di modulazione sincrona (lock-in) sulla corrente ionica di rivelazione del FAIMS. Sulla base dei risultati di questi studi, è stato possibile migliorare sensibilmente il limite di rivelazione (LoD) del FAIMS. I valori di LoD per acetone, estrapolati per il sistema FAIMS sviluppato in questa tesi, sono 900 e 40 ppt per un flusso di gas portante pari rispettivamente a 3.05 e $9.5 \mathrm{l} / \mathrm{min}$. Questi risultati sono simili o leggermente migliori dei livelli trovati all'avanguardia per FAIMS autonomi già pubblicati in letteratura.

Parole chiave: Spettrometria di mobilità ionica, Mobilità ionica, Detezione chimica, Filtraggio di ioni, Detezione di ioni, Limite di rivelabilità, Fotoionizzazione UV, Amplificatore a basso rumore, Detezione lock-in. 


\section{Contents}

Abstract (English/Italian) $\quad$ i

List of figures vii

List of tables $\quad$ Xv

1 Introduction 1

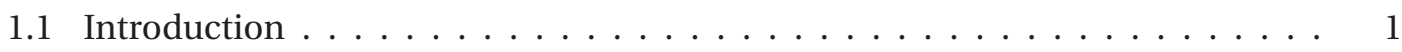

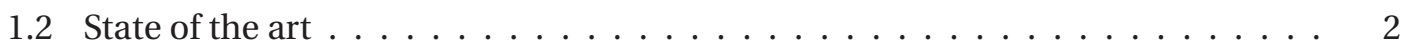

1.2.1 Photo Ionization Detectors (PID) . . . . . . . . . . . . . . . 2

1.2 .2 Mass spectrometers $\ldots \ldots \ldots \ldots \ldots \ldots \ldots \ldots \ldots$

1.2.3 Micro mass spectrometers $(\mu \mathrm{MS}) \quad \ldots \ldots \ldots \ldots$

1.2.4 High-Field Asymmetric Waveform Ion Mobility Spectrometry (FAIMS) . 3

1.2.5 Tandem Ion-mobility spectrometry-mass spectrometry (IMS-MS) . . . . 5

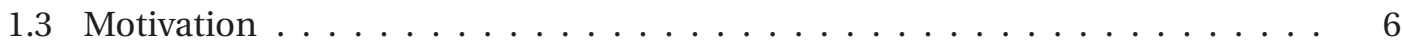

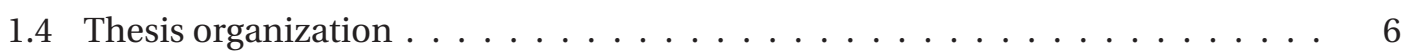

2 Ion Mobility: Theory and Simulation $\quad 7$

2.1 UV Photoionization . . . . . . . . . . . . . . . 7

2.2 Ion mobility dependency on the electric field $\ldots \ldots \ldots \ldots$

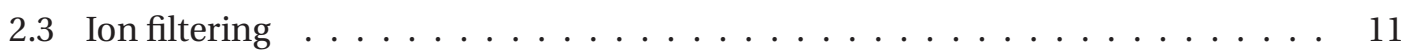

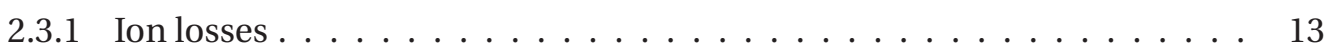

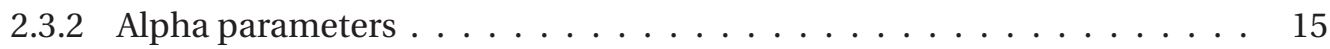

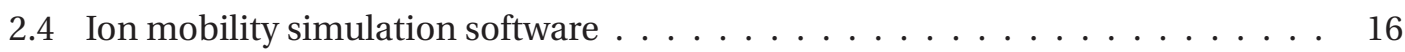

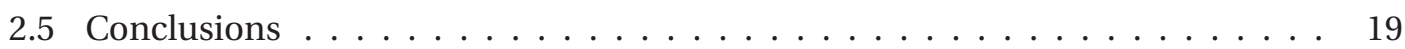

3 Design and Fabrication of the FAIMS system 21

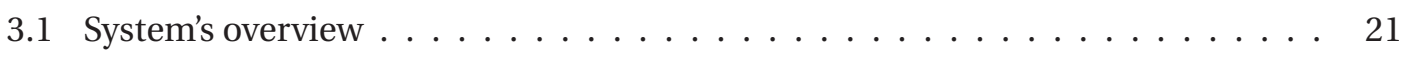

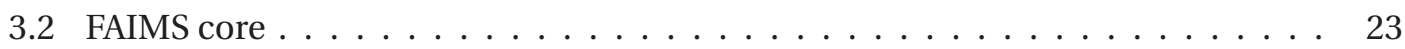

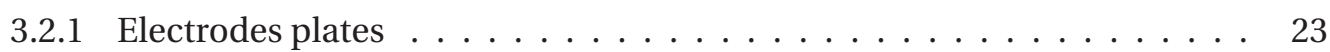

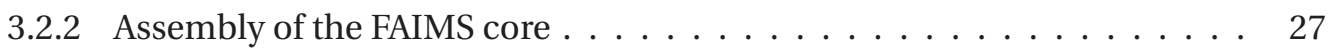

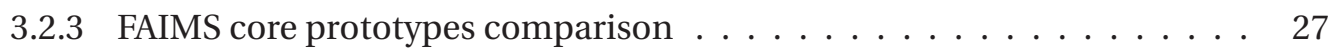

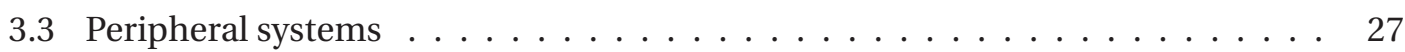

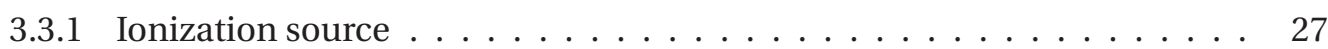




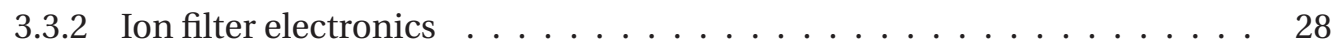

3.3.3 Compensation Voltage electronics . . . . . . . . . . . . . . . . 31

3.3 .4 Ion detector $\ldots \ldots \ldots \ldots \ldots \ldots \ldots \ldots \ldots \ldots \ldots \ldots$

3.3 .5 Lock-in Amplifier . . . . . . . . . . . . . . . . . . . . . . 35

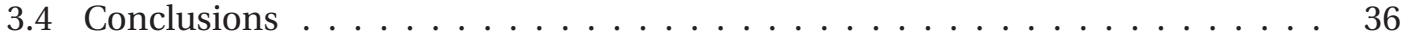

4 Experimental results $\quad 39$

4.1 Ionization rate . . . . . . . . . . . . . . . . . . 39

4.1.1 Effects of the UV source input current on the ionization efficiency . . . . 39

4.1.2 Effects of Carrier gas composition on the ionization efficiency . . . . . . 41

4.1.3 Effects of the carrier gas flow rate on the ionization efficiency . . . . . . 4 43

4.1 .4 Ion losses . . . . . . . . . . . . . . . . . . . . 44

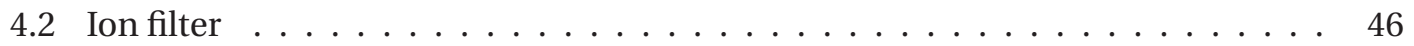

4.2 .1 Noise reduction . . . . . . . . . . . . . . . . . . . . 46

4.2.2 Ion filtering at different separation voltages $\ldots \ldots \ldots$. . . . . . 49

4.2 .3 Humidity effects in the ion separation . . . . . . . . . . . . . . 52

4.2.4 Influence of the filtering frequency on the ion separation $\ldots . . \ldots 53$

4.2.5 Effects of the carrier gas pressure on the ion separation $\ldots . \ldots 53$

4.2.6 Effects of the carrier gas flow rate on the ion separation . . . . . . . . 55

4.2 .7 Effects of the filter size on the ion separation . . . . . . . . . . . 59

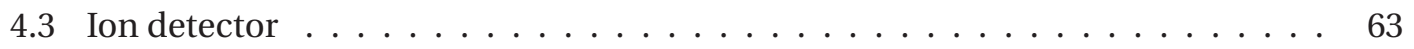

4.3.1 Effect of the deflection voltage on the ion capture rate . . . . . . . . 63

4.3 .2 Lock-in detection . . . . . . . . . . . . . . . . . . . . . . 64

4.4 Limit of detection $(\mathrm{LoD}) \ldots \ldots \ldots \ldots \ldots \ldots \ldots$

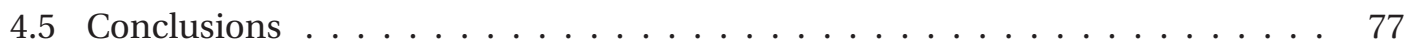

5 Conclusions and Outlook $\quad 81$

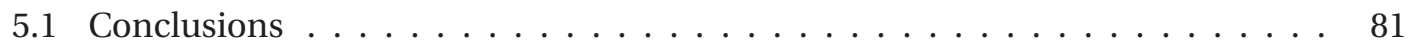

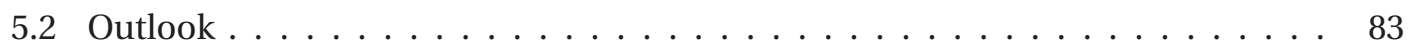

A Flowchart of the ion mobility simulation software 85

$\begin{array}{lll}\text { B FAIMS electrode plates designs } & 87\end{array}$

C LabVIEW block diagrams of the developed signal processing and user interface software

$\begin{array}{ll}\text { D Lock-in detection experimental parameters } & 97\end{array}$

$\begin{array}{ll}\text { Bibliography } & 102\end{array}$

$\begin{array}{ll}\text { Acknowledgements } & 103\end{array}$

$\begin{array}{lr}\text { Curriculum Vitae } & 105\end{array}$ 


\section{List of Figures}

1.1 Characteristic subsystems of a basic IMS. . . . . . . . . . . . . . . . . 1

1.2 Basic schematic of a Photoionization detector (PID) highlighting the main components of the UV ionization source and the ion detector. Image reproduced from the RAE Systems' PID Handbook [1] . . . . . . . . . . . . . . . . . . . . .

1.3 Schematic of the Planar Integrated Micro Mass Spectrometer (PIMMS) developed by Prof. Müller's group at the TUHH [2]. The PIMMS systems are batch fabricated using MEMS technologies and have a total area of about $1 \mathrm{~cm}^{2}$. . .

1.4 Schematic of the Planar Field Asymmetric Ion Mobility Spectrometer (p-FAIMS) depicting its four main subsections: desorption region, ionization region, ion filter and ion detector. . . . . . . . . . . . . . . . . . . . .

2.1 Examples of the light absorption cross sections of acetone (a) and nitrogen (b) at room temperature for different wavelengths. Plots taken from the MPI-Mainz UV/VIS Spectral Atlas of Gaseous Molecules [3]. The red lines indicate the light wavelengths emitted by the implemented UV ionization source, i.e. 117 and 124

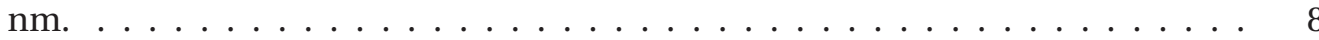

2.2 Ion mobility vs $\mathrm{E} / \mathrm{p}$ for different compounds in nitrogen. Image reproduced from A. Tyndall [4]. . . . . . . . . . . . . . . . . . . . . 10

2.3 Ion mobility ratio for three characteristic ion types. . . . . . . . . . . . . . 10

2.4 Representation of the ion trajectories of two different ions under high and low electric fields with opposite polarities. In the first case (yellow) the ion mobility doesn't change, thus the ion returns to its initial position between the electrodes. In the second case (red) the ion mobility decreases at high electric field, therefore, the ion position between the electros is shifted by $\Delta \mathrm{d} . \ldots \ldots \ldots \ldots$

2.5 Representation of the FAIMS ion filtering principle: The ions follow different trajectories depending on their mobility variation at low and high electric fields. The compensated ions (yellow) remain in the ion channel until the detection region while the non-compensated ions (red and blue) get deflected towards the electrodes. . . . . . . . . . . . . . . . . . . . . . . . . . . 12

2.6 Examples of FAIMS spectra for different compounds presented by R.A. Miller at al. [5]. The ion filtering was performed at 900Vpp. . . . . . . . . . . . .

2.7 Separation waveform coefficients for common FAIMS filtering signals. Image reproduced from E. Krylov at al. [6] . . . . . . . . . . . . . . . . . . . . . 16 
2.8 Interface window of the developed ion mobility simulation software; , the main input/output regions are outlined in red (Simulated ions' trajectories), black (Parameter's input fields) and blue (Simulated ion current spectra). . . . . . . .

2.9 Comparison between the acetone's simulated and experimental ion spectra at different separation voltages. All measurements were performed with a $250 \mu \mathrm{m}$ gap FAIMS at a concentration of $3 \mathrm{ppm}$ in a nitrogen. The carrier gas flow was 9 liters per minute and relative humidity was $60 \%$. . . . . . . . . .

2.10 Compensation Voltage shift-vs.- separation voltage plots for acetone at different RH levels. The simulated and experimental data are compared with a theoretical fitting curve. The experimental data in plot (a) was obtained in our lab while the experimental data in plot (b) was taken from an external paper [7]. . . . . . . .

3.1 Schematics of the FAIMS including peripheral systems. Two different ionization sources are represented: (a) corona discharge and (b) UV source ionization. . .

3.2 Schematic of the experimental FAIMS measurements setup. A commercial $\mathrm{V}-\mathrm{OVG}$ calibration gas generator from Owlstone Ltd. is used as the analyte desorption/injection system. Different sensors are implemented to monitor the experimental conditions, e.g. gas flow, humidity, temperature, pressure. . . . .

3.3 Exploded view of the three fabricated FAIMS cores (dimensions in $\mathrm{mm}$ ). The design in a) has a corona discharge ionization system while b) and c) have UV ionization sources. The electrode plates in were fabricated in alumina, (a) and (b), and in FR4,(c). Two needle holders were made in model a) with different sample input geometries. . . . . . . . . . . . . . . .

3.4 Fabricated electrode plates with alumina substrate. The electrodes are screen printed on the substrate using silver-palladium ink. . . . . . . . . . .

3.5 Fabricated PCB electrode plates for FAIMS (a,b) and ion losses measurements (c). The plates in (a) have a similar geometry to those fabricated in alumina, with vias connecting the electrodes in the inner face to the connection pads on the backside. The plates in (b) and (c) have conductive layer only on the inner face and the connection pads are placed at the same side of the electrodes to simplify the connection. Also, (b) and (c) only have the tin layer on the connection area, for the electrodes section the copper layer was left uncovered. . . . . . . . . . 26

3.6 Assembled FAIMS cores using the fabricated PCB and alumina electrode plates. 27

3.7 FAIMS system including the protection case and the UV source. . . . . . . . . 28

3.8 FAIMS system with the protection case and the corona discharge ionization

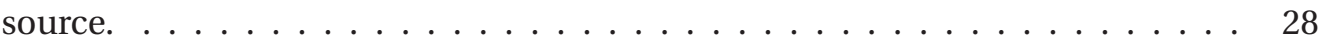

3.9 Schematic of the nMOS inverter circuit for the ion filter. The inserted waveform diagram shows how the input signal (0) is split in two opposite signals (A,B) and the delay between transitions is added ( $\mathrm{C}$ to $\mathrm{F}) . \ldots \ldots \ldots$

3.10 Schematic of the compensation voltage amplifier including the stage to add the modulation signal. . . . . . . . . . . . . . . . 
3.11 Picture of the fabricated high voltage and compensation voltage circuits for the ion filtering. Both circuits are shielded inside a metallic box for protection and to reduce the coupled noise into the detected signal. . . . . . . . . . . . . . . 32

3.12 Schematic of the low noise current amplifier circuit. . . . . . . . . . . . . . . 33

3.13 Voltage noise versus frequency behavior of the LMC6001 Ultra-Low Input Current Amplifier [8] . . . . . . . . . . . . . . . . . . . . .

3.14 Comparison of the current noise spectral density of the fabricated amplifier with different gains. The dashed lines represent the calculated theoretical input referred noise using Equation 3.1. These measurements were performed with zero ion current. . . . . . . . . . . . . . . . . . . . . 34

3.15 Basic blocks diagram of a lock-in amplifier. . . . . . . . . . . . . . . . 35

3.16 Schematic of the modified FAIMS setup with synchronous detection. . . . . . . 36

3.17 Different processing steps for the acquired lock-in signal. . . . . . . . . . . . . 36

3.18 Fabricated FAIMS system with the shielding covers. . . . . . . . . . . . . . . . 37

4.1 Variation in the UV lamp's output power and the detected ion current amplitude and FWHM at different input currents. The dashed lines between the points are only visual guides, not fitted lines or theoretical modeling of the physical behavior. 40

4.2 Detected acetone spectra using different UV source input currents. The spectra in (a) were not filtered by the FAIMS high voltage signal. The spectra in (b) were filtered by a $700 \mathrm{~V}_{\mathrm{PP}}$ high voltage signal at $1 \mathrm{MHz}$ and $33 \%$ duty cycle. Each curve is the average of 10 measurements under the same conditions. . . . . . . . . . .

4.3 Detected acetone spectra using nitrogen as a carrier gas with different levels of humidity. The measurements were taken without the ion filter signal. Each curve is the average of 10 measurements under the same conditions. . . . . . .

4.4 Detected acetone spectra at different carrier gas flow rates. The analyte flow is constant for all measurements: $1.2 \times 10^{16}$ acetone molecules per second. The measurements were taken at high (b) and low (a) humidity levels without the ion filter signal. Each curve is the average of 10 measurements under the same

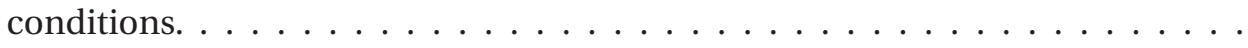

4.5 Variation of the detected ion peak's amplitude and FWHM at different carrier gas flow rates and a constant analyte flow. Each point is the average of 10 measurements under the same conditions. . . . . . . . . . . . . . . . 44

4.6 Schematic of the ion losses experimental setup. . . . . . . . . . . . . . . . 45

4.7 Reduction in the ion current inside the FAIMS channel due to the ions recombination and diffusion. Each curve was taken at different conditions, modifying only one parameter at the time. The distance in the $\mathrm{X}$ axis is measured from the center of the ionization region to the center of the detection region. The dashed lines between the points are only visual guides, not fitted lines or theoretical modeling of the physical behavior. Each point is the average of 10 measurements under the same conditions. . . . . . . . . . . . . . . . . . . . . 46 
4.8 Influence of the low pass filter's cutoff frequencies in the HV ion filter waveform. Lower $f_{c}$ decrease the signal noise but also deforms the waveform. The signals in all measurements were set to $300 \mathrm{~V}_{\mathrm{PP}}$ at $1 \mathrm{MHz}$ and $33 \%$ duty cycle. The cutoff frequencies $\left(f_{c}\right)$ were calculated from the measured HV waveforms. . . . . . .

4.9 Effects of the LP filter's cutoff frequency on the detected acetone FAIMS spectra. The detected spectra are shown in (a) and the variation in the ions peak's amplitude and CV shift are summarized in (b). All measurements were perform with a FAIMS ion filtering of $700 \mathrm{~V}_{\mathrm{PP}}$ at $1 \mathrm{MHz}$ and $33 \%$ duty cycle. Air was used as carrier gas at room conditions, i.e. $1 \mathrm{~atm} ., 22{ }^{\circ} \mathrm{C}$ and $25 \%$ relative humidity. The dashed lines between the points are only visual guides, not fitted lines or theoretical modeling of the physical behavior. . . . . . . . . . . . . .

4.10 Comparison between the NSD before (black) and after (red) implementing the LP filter and the shielding boxes. . . . . . . . . . . . . . . . . . . . 49

4.11 Effects of the separation voltage (HV) on the detected acetone spectra with a concentration of $9.5 \mathrm{ppm}$. The filter HV levels were increased from 0 to 750 $\mathrm{V}_{\mathrm{PP}}$ at $1 \mathrm{MHz}$ and $33 \%$ duty cycle. In plot (a), the detected spectra at different HV levels are displayed as different curves. In plot (b), the detected spectra are placed along the "Y" axis and the ion current is represented with a color scale. .

4.12 Effects of the separation voltage (HV) on the detected o-xylene spectra with a concentration of $2.7 \mathrm{ppm}$. The filter HV levels were increased from 0 to 750 $\mathrm{V}_{\mathrm{PP}}$ at $1 \mathrm{MHz}$ and $33 \%$ duty cycle. In plot (a), the detected spectra at different HV levels are displayed as different curves. In plot (b), the detected spectra are placed along the "Y" axis and the ion current is represented with a color scale. .

4.13 (a) Effects of the humidity levels in the detected acetone spectra. (b) Compensation Voltage shift -vs- separation voltage plots for acetone at different $\mathrm{RH}$ levels. . . . . . . . . . . . . . . . . . . . . . . .

4.14 Effects of the HV waveforms' frequency on the FAIMS signal. (a) Detected spectra of $2 \mathrm{ppm}$ of acetone at different filtering frequencies ( 1 scan per plot). The separation voltage was set to $700 V_{P P}$ with a $33 \%$ duty cycle. Nitrogen was used as carrier gas with a flowrate of $3.06 \mathrm{l} / \mathrm{min}$ and relative humidity of $5 \%$. (b) Normalized variation of the ion peak's amplitude and CV position. . . . . . . .

4.15 Effects of the carrier gas pressure on the FAIMS signal. (a) Detected spectra of $9.5 \mathrm{ppm}$ of acetone at different pressures (1 scan per plot). Nitrogen was used as carrier gas with a flowrate of $3.06 \mathrm{l} / \mathrm{min}$ and relative humidity of $5 \%$. The pressure was changed from 0.33 to $1 \mathrm{~atm}$. The separation voltage was set to 300 $V_{P P}$ with a $33 \%$ duty cycle. (b) Normalized variation of the detected spectra's characteristic parameters. The dashed lines between the points are only visual guides, not fitted lines or theoretical modeling of the physical behavior. . . . . . 
4.16 Effects of the carrier gas flow rate on detected acetone spectra (a). The analyte flow is constant for all measurements: $1.2 \times 10^{16}$ acetone molecules per second (10 scans per plot). Nitrogen was used as carrier gas with flow rates from 2.1 to $12.3 \mathrm{l} / \mathrm{min}$ and relative humidity of $5 \%$. The separation voltage was set to $700 \mathrm{~V}_{\mathrm{PP}}$ at $1 \mathrm{MHz}$ and $33 \%$ duty cycle. (b) Normalized variation of the detected spectra's characteristic parameters. The dashed lines between the points are only visual guides, not fitted lines or theoretical modeling of the physical behavior. . . . . .

4.17 Normalized acetone spectra taken at carrier gas flow rates from 2.1 to $14.3 \mathrm{l} / \mathrm{min}$. The position of the main ion peak remains relatively constant at different flow rates. Nevertheless, the CV shift the secondary ion peak decreases with the flow, until it merges with the main peak. . . . . . . . . . . . . . . . . . .

4.18 Effects of the carrier gas flow rate on detected acetone spectra (a). The analyte flow is constant for all measurements: $1.2 \times 10^{16}$ acetone molecules per second (10 scans per plot). Nitrogen was used as carrier gas with flow rates from 2.1 to $14.3 \mathrm{l} / \mathrm{min}$ and relative humidity of $60 \%$. The separation voltage was set to $700 \mathrm{~V}_{\mathrm{PP}}$ at $1 \mathrm{MHz}$ and 33\% duty cycle. (b) Normalized variation of the detected spectra's characteristic parameters. The dashed lines between the points are only visual guides, not fitted lines or theoretical modeling of the physical behavior. 58

4.19 Measured differential pressure, between the ions' channel input and output, at gas flow rates from 2.1 to $15 \mathrm{l} / \mathrm{min}$. The dashed lines between the points are only visual guides, not fitted lines or theoretical modeling of the physical behavior. .

4.20 Schematics of the implemented electrode plates with different ion filter (green) lengths and constant distance between the ionization region (purple) and the ion detector (red). All dimensions are in $\mathrm{mm} . \quad \ldots$. . . . . . . . . . . . . .

4.21 Effects of the ion filter length on detected acetone (9.5 ppm) spectra at different separation voltages, i.e. $0 \mathrm{~V}$ (a), $300 \mathrm{~V}_{\mathrm{PP}}$ (b), $500 \mathrm{~V}_{\mathrm{PP}}$ (c) and $700 \mathrm{~V}_{\mathrm{PP}}$ (d). The nitrogen flow rate was set to $3.06 \mathrm{l} / \mathrm{min}$ with $5 \%$ relative humidity. The applied $\mathrm{HV}$ waveform had a frequency of $1 \mathrm{MHz}$ and $33 \%$ duty cycle. Each curve is the average of 10 measurements under the same conditions. . . . . . . . . . . . .

4.22 Normalized variation of acetone spectra's amplitude and resolution at different ion filter lengths and separation voltages. The length of the ion filter was varied from 12 to $5 \mathrm{~mm}$. The nitrogen flow rate was set to $3.06 \mathrm{l} / \mathrm{min}$ with $5 \%$ relative humidity. The applied $\mathrm{HV}$ waveform was changed from 0 to $700 \mathrm{~V}_{\mathrm{PP}}$ with a frequency of $1 \mathrm{MHz}$ and 33\% duty cycle. Each point is the average of 10 measurements under the same conditions. The dashed lines between the points are only visual guides, not fitted lines or theoretical modeling of the physical behavior. . . . . . . . . . . . . . . . . . . . . . . 
4.23 Effects of the ion filter length on detected acetone spectra. The length of the ion filter was varied from 15 to $5 \mathrm{~mm}$. The nitrogen flow rate was set to 3.06 $1 /$ min with $75 \%$ relative humidity. The ion filter was performed with a $700 V_{\mathrm{PP}}$ waveform with a frequency of $1 \mathrm{MHz}$ and $33 \%$ duty cycle. Each curve is the average of 10 measurements under the same conditions. The dashed lines between the points are only visual guides, not fitted lines or theoretical modeling of the physical behavior. . . . . . . . . . . . . . . . . . . . .

4.24 Noise spectral density from the low noise current amplifier measured at filterdetector distances from 5 to $10 \mathrm{~mm}$. The ion filter waveform was set to 700 $\mathrm{V}_{\mathrm{PP}}$ at $1 \mathrm{MHz}$ and $33 \%$ duty cycle. An additional measurement (black curve) with the ion filter off is presented as reference. Each curve is the average of 10 measurements under the same conditions. . . . . . . . . . . . . . . . .

4.25 Variation of the detected acetone ion peak at different deflection voltage levels. The analyte flow was kept constant to $1.2 \times 10^{16}$ molecules per second. The nitrogen flow was set to 3.05 (a,b) and 11 (c,d) 1/min with a humidity level of $3 \%$. The measurements in $(\mathrm{a}, \mathrm{c})$ were taken without the ion filter's HV signal while the one in (b,d) were taken with a filter voltage of $700 \mathrm{~V}_{\mathrm{PP}}$ at $1 \mathrm{MHz}$ and $33 \%$ duty cycle. The black symbols represent the experimental data and the red ones the average value. The dashed lines between the points are only visual guides, not fitted lines or theoretical modeling of the physical behavior. . . . . . . . . . .

4.26 Detected acetone spectra using the FAIMS + lock-in amplifier setup at different modulation amplitudes. Plot (a) shows the original signals from the Lock-in amplifier while plot (b) the integrated lock-in spectra. The modulation frequency was set to $7.12 \mathrm{~Hz}$. The carrier gas and the acetone flowrates were kept constant at $3.05 \mathrm{l} / \mathrm{min}$ and $2.5 \times 10^{15}$ molecules/second respectively. The ion filtering was performed at $700 \mathrm{VPP}, 1 \mathrm{MHz}$ and $33 \%$ duty cycle. Additional experimental parameters are summarized in Appendix D. . . . . . . . . . . . . . . .

4.27 Detected acetone spectra using the FAIMS + lock-in amplifier setup at different modulation amplitudes. Plot (a) shows the original signals from the Lock-in amplifier while plot (b) the integrated lock-in spectra. The modulation frequency was set to $512.3 \mathrm{~Hz}$. The carrier gas and the acetone flowrates were kept constant at $3.05 \mathrm{l} / \mathrm{min}$ and $2.5 \times 10^{15}$ molecules/second respectively. The ion filtering was performed at $700 \mathrm{~V}_{\mathrm{PP}}, 1 \mathrm{MHz}$ and $33 \%$ duty cycle. Additional experimental parameters are summarized in Appendix D. . . . . . . . . . 
4.28 Variation of the detected signal's amplitude (a,b) and FWHM (c,d) at different modulation amplitudes with low (black) and high (red) modulation frequency: The left side plots $(\mathrm{a}, \mathrm{c})$ show the behavior of the original signals from the Lock-in amplifier while the right side plots $(b, d)$ show the behavior of integrated spectra. The carrier gas and the acetone flowrates were kept constant at $3.05 \mathrm{l} / \mathrm{min}$ and $2.5 \times 10^{15}$ molecules/second respectively. The ion filtering was performed at $700 \mathrm{~V}_{\mathrm{PP}}, 1 \mathrm{MHz}$ and 33\% duty cycle. Additional experimental parameters are summarized in Appendix D. The dashed lines between the points are only visual guides, not fitted lines or theoretical modeling of the physical behavior. . . . . .

4.29 Detected acetone spectra using the FAIMS + lock-in amplifier setup at different modulation frequencies. The acetone flowrate was kept constant at $2.5 \times 10^{15}$ molecules/second. Two sets of measurements are presented: the measurements in (a) were taken with a carrier gas flow of $3.05 \mathrm{l} / \mathrm{min}$ while the measurements in (b) at a flowrate of $9.5 \mathrm{l} / \mathrm{min}$. The ion filtering was performed at $700 \mathrm{~V}_{\mathrm{PP}}, 1$ $\mathrm{MHz}$ and 33\% duty cycle. Additional experimental parameters are summarized in Appendix D . . . . . . . . . . . . . . . . . . . . . . . . . . . .

4.30 Normalized variation versus the modulation frequency of the ion peak's amplitude (a) and FWHM (b) at different gas flowrates, and modulation amplitudes. The acetone flowrate was kept constant at $2.5 \times 10^{15}$ molecules/second. The ion filtering was performed at $700 \mathrm{~V}_{\mathrm{PP}}, 1 \mathrm{MHz}$ and $33 \%$ duty cycle. Additional experimental parameters are summarized in Appendix D. The dashed lines between the points are only visual guides, not fitted lines or theoretical modeling of the physical behavior. . . . . . . . . . . . . . . . . .

4.31 Comparison between the detected acetone spectra with and without lock-in detection. (a) The modulation for the measurements with $\mathrm{N}_{2}$ flow of $3.05 \mathrm{l} / \mathrm{min}$ was done at $7.123 \mathrm{~Hz}$ with $0.212 \mathrm{~V}_{\mathrm{RMS}}$ amplitude. (b) The modulation for the measurements with $\mathrm{N}_{2}$ flow of $9.5 \mathrm{l} /$ min was performed at $25.12 \mathrm{~Hz}$ with 0.812 $\mathrm{V}_{\mathrm{RMS}}$ amplitude. . . . . . . . . . . . . . . . . .

4.32 SNR variation at different modulation frequencies for gas flows of 3.05 (a) and 9.5 (b) 1/min. The dashed red lines represent the SNR level for FAIMS measurements, under the same conditions, without the lock-in amplifier. Additional experimental parameters are summarized in Appendix D . . . . . . . . . . .

4.33 Acetone spectra's SNR variation at different modulation amplitudes with a modulation frequency of $7.12 \mathrm{~Hz}$. The dashed orange line represents the SNR level for FAIMS measurements, under the same conditions, without the lock-in amplifier. The carrier gas and the acetone flowrates were kept constant at $3.05 \mathrm{l} / \mathrm{min}$ and $2.5 \times 10^{15}$ molecules/second respectively. The ion filtering was performed at $700 \mathrm{~V}_{\mathrm{PP}}, 1 \mathrm{MHz}$ and $33 \%$ duty cycle. Additional experimental parameters are summarized in Appendix D . . . . . . . . . . . . . . . . . . . . . . . 
4.34 Acetone spectra detected at concentrations from 26 to $286 \mathrm{ppb}$ with the ion filter OFF (a) and $\mathrm{ON}(\mathrm{b})$. The selected carrier gas was nitrogen with $5 \%$ relative humidity and $3.05 \mathrm{l} / \mathrm{min}$ flowrate. The ion filtering in (b) was performed at 700 PP, $1 \mathrm{MHz}$ and $33 \%$ duty cycle. The ion current was measured with a sampling frequency of $12.3 \mathrm{kHz}$, a sampling rate of 10 points/volt and an averaging time of 0.1 seconds/ point, i.e. $\sim 20$ seconds per scan. . . . . . . . . . . .

4.35 Acetone spectra detected at concentrations from 8 to $88 \mathrm{ppb}$ with the ion filter OFF (a) and ON (b). The selected carrier gas was nitrogen with $5 \%$ relative humidity and $9.5 \mathrm{l} / \mathrm{min}$ flowrate. The ion filtering in (b) was performed at 700 $\mathrm{V}_{\mathrm{PP}}, 1 \mathrm{MHz}$ and $33 \%$ duty cycle. The ion current was measured with a sampling frequency of $12.3 \mathrm{kHz}$, a sampling rate of 10 points/volt and an averaging time of 0.1 seconds/ point, i.e. $\sim 20$ seconds per scan. . . . . . . . . . .

4.36 Variation of the ions peak amplitude at different acetone concentration levels. The selected carrier gas was nitrogen with $5 \%$ relative humidity and flowrates of 3.05 (a) and 9.5 (b) 1/min. The measurements in black were performed with the ion filter OFF and the red ones with the filter $\mathrm{ON}$ at $700 \mathrm{~V}_{\mathrm{PP}}, 1 \mathrm{MHz}$ and $33 \%$ duty cycle. The red and black symbols show the experimental data, the red and black lines represent the linear fitting of the experimental measurements and the dashed lines the minimum ion current detection level with different measurement parameters. . . . . . . . . . . . . . . . . 76

B.1 Drawings of the upper alumina electrodes plates with filter electrodes from 8 to $15 \mathrm{~mm}$ long (Dimensions in $\mathrm{mm}) . \ldots \ldots \ldots . \ldots \ldots$

B.2 Drawings of the bottom alumina electrodes plates with filter electrodes from 8 to $15 \mathrm{~mm}$ long (Dimensions in $\mathrm{mm}$ ). . . . . . . . . . . . . . . . . . 89

B.3 Drawings of the alumina spacer. The standard implemented thickness was 250 $\mu \mathrm{m}$. However, spacers with thicknesses of 170 and $500 \mu \mathrm{m}$ were also fabricated (Dimensions in $\mathrm{mm}) \ldots \ldots \ldots \ldots \ldots$. . . . . . . . . . . . . . . . . . .

B.4 Drawings of the top and bottom PCB electrodes plates. . . . . . . . . . . 91

B.5 Drawings of the top and bottom PCB electrodes plates for ion losses measurement. 92 


\section{List of Tables}

3.1 Summary of the tested low noise current amplifier parameters . . . . . . . . 32

4.1 Absorption cross-sections of different gases used in FAIMS for the wavelength of the implemented UV source $(123.6 \mathrm{~nm})[3] \ldots \ldots$. . . . . . . . . . 42

4.2 Summary of the tested LP filter components. The cutoff frequencies $\left(f_{c}\right)$ were calculated from the measured HV waveforms (The circuit schematics are included in Figure 3.9). . . . . . . . . . . . . . . . . . . . . 47

4.3 Comparizon between the graphically determined and the calculated LoD levels at different carrier gas flowrates, with and without ion filtering. Measurement conditions: sampling frequency of $12.3 \mathrm{kHz}$, sampling rate of 10 points/volt, averaging time of 0.1 seconds/ point and one scan per measurement. . . . . . .

4.4 Calculated LoD of the developed FAIMS system using the lock-in amplifier setup. The LoD was calculated based on experimental results at low and high gas flowrates with and without ion filtering. The data is converted to different analyte flow units to facilitate the comparison with other publications and techniques.

D.1 Experimental parameters for the lock-in analysis. . . . . . . . . . . . . . . . 97 



\section{Introduction}

\subsection{Introduction}

Ion Mobility Spectrometry (IMS) is a commonly used technique for ion separation in the gaseous phase at room conditions. Its filtering principle is based on the variations in ion mobilities under different electric fields. IMS was developed in the final decades of the last century as a fast and simple method to detect and identify volatile organic compounds (VOC), mainly for security and military applications. In the following years, more refined devices have been developed based on the same IMS principles. Today analyzers are handheld, portable and capable to perform measurements in ambient conditions with accurate detection levels. This has widely increased the IMS applications range, reaching fields like medicine, biology, industrial hygiene and safety, among others $[9,10,11]$.

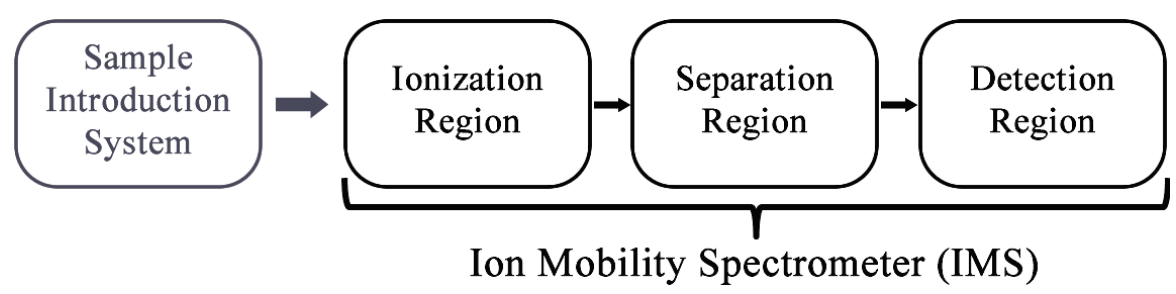

Figure 1.1: Characteristic subsystems of a basic IMS.

A basic IMS is formed by four subsystems, as depicted in Figure 1.1: the sample introduction system, the ionization region, the ion drift channel or separation region and the detection region. New advances in engineering and electronics and more efficient drift tube designs have led to several configurations of these four subsystems and a large amount of acronyms, e.g. FAIMS, DTIMS, TWIMS, OLIMS, etc. Three of the most common approaches are: time of flight IMS, aspirator IMS and FAIMS. In Time of flight IMS, ions are injected into the drift region at a given time interval. The ion packet is accelerated towards the detector by a voltage gradient. Finally, ion characteristics can be associated to its drift velocity.

In aspirator IMS, ions are driven through a parallel plate structure by a gas flow towards the detector. Meanwhile, a relatively low electric field is applied between these plates to generate an ion displacement perpendicular to the flow direction. The position where the ions hit the detection plate depends of the applied electric field and the ion mobility coefficient. The 
main advantages of this configuration are its simplicity and the possibility of continuous monitoring, its main disadvantages are its low resolution and specificity [12, 13].

High-Field Asymmetric Waveform Ion Mobility Spectrometry (FAIMS) applies a train of pulses (positive short duration high voltage mixed with negative long duration low voltage) to the ion filter to generate a variable electric field. The system exploits the variation in the ion mobility for high and low electric fields to separate the desired ions and discard the others. Finally, the filtered ions are deflected towards the detection electrodes and registered by a weak current detection system $[14,15]$.

\subsection{State of the art}

There is a growing interest in VOC detection in many fields. This has led to the development of a wide range of detection systems, from very simple approaches, e.g. Photo Ionization Detectors (PID), to very complex ones, like Tandem mass spectrometry. In this section, examples of state of the art techniques relevant in the scientific and industrial fields are presented.

\subsubsection{Photo Ionization Detectors (PID)}

A PID is a simple and inexpensive VOC detector. It consists of an UV ionization source attached to a gas chamber, where the sample is introduced. Inside the gas chamber, there is an electrode array that deflects and captures the ionized VOCs. The ion current is then amplified and displayed/recorded. Figure 1.2 shows a schematic of a PID's basic components. PIDs lack ion filters or any other ion discerning system. The PID's UV sources emit photons in the

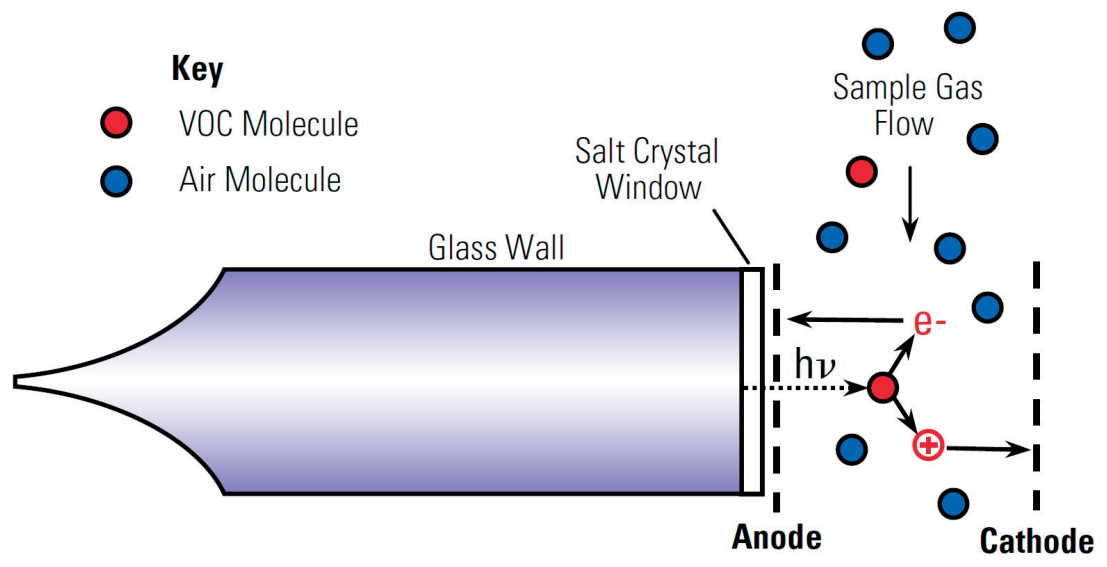

Figure 1.2: Basic schematic of a Photoionization detector (PID) highlighting the main components of the UV ionization source and the ion detector. Image reproduced from the RAE Systems' PID Handbook [1].

Vacuum Ultraviolet region (VUV). Different gases and mixtures are used to optimize the output wavelengths and intensity, e.g. $\mathrm{Kr}, \mathrm{Ar}$, Xe. The lamp's wavelength is set so that the ionization potential is high enough to ionize most VOCs but lower than the ionization threshold of common air elements, such as $\mathrm{N}_{2}$ and $\mathrm{O}_{2}$. Commercial PID systems have detection limits in the sub-ppb range [1]. 


\subsubsection{Mass spectrometers}

Mass spectrometry (MS) is one of the most accurate techniques available for chemical analysis today [16]. It is also one of the most sensitive tools, with detection limits in the yoctomoles range having been reported [17]. Out of all the techniques analyzed in this report, MS is the only one capable of localized chemical imaging with spatial resolution in the $\mu \mathrm{m}$ and $\mathrm{nm}$ range: different methods have been proposed for chemical imaging using laser and thermal based desorption techniques coupled to commercial MS systems. [18, 19, 20].

The main drawback of MS is its complexity: MS measurements should be performed in high vacuum or in a special media, the samples require extensive preparation, etc. Also, commercial mass spectrometers are large, heavy and expensive. This limits the application of mass spectrometry systems in different areas due to environmental, physical and cost constraints. Therefore, miniaturization is one of the main topics of research in modern mass spectrometry $[2,21]$.

\subsubsection{Micro mass spectrometers ( $\mu \mathrm{MS})$}

Micro mass spectrometers have several advantages compared to a normal sized spectrometer. Small MS devices can be produced a much lower costs and require much less power to work. Also, the media requirements are more flexible since the necessary ion mean free path scales with size. Finally, miniaturized systems can be handheld, allowing in-situ measurements. Nevertheless, the miniaturization and simplification of the MS also reduces its sensitivity. The limit of detection (LoD) of micro mass spectrometers is in the order of tens of ppm [2].

There are several groups working on $\mu \mathrm{MS}[2,21,22]$ and some have already commercially available systems, for example the Microsaic Systems Ltd in London. One of the most interesting prototypes, denominated PIMMS (Planar Integrated Micro Mass Spectrometer), is currently under development in the Microsystem Technology laboratory at the Hamburg University of Technology (TUHH). In the PIMMS, all components of a classical mass spectrometer, i.e., ion source, ion separation system and ion detector, are integrated on a single chip of about 1 $\mathrm{cm}^{2}$ (Figure 1.3). The sample is introduced directly into the ion source where it is ionized by electron impact. The ion source is formed by the electrons' source and the ionization chamber. After ionization, a set of ion optics extract and accelerate the ions in a focused beam towards the mass analyzer. In the mass analyzer, ions are exposed to an electric field perpendicular to their movement direction. Only the ions with a specific velocity are able to pass through the separator channel, all other ions are deflected. This velocity is given by the frequency of the rectangular shaped signals driving the separator electrodes. An energy filter after the ion separator filters the ions according to their kinetic energy. Finally, the ions are detected by a Faraday cup. The LoD of this $\mu \mathrm{MS}$ is about $100 \mathrm{ppm}[2,21]$.

\subsubsection{High-Field Asymmetric Waveform Ion Mobility Spectrometry (FAIMS)}

FAIMS systems are handheld, have good sensitivity and resolution levels and are relatively inexpensive. These characteristics make FAIMS a very interesting chemical characterization technique for many research groups around the world, especially in Asia and North America $[11,23,24,25]$. Each group makes their own contributions and improvements to the system 


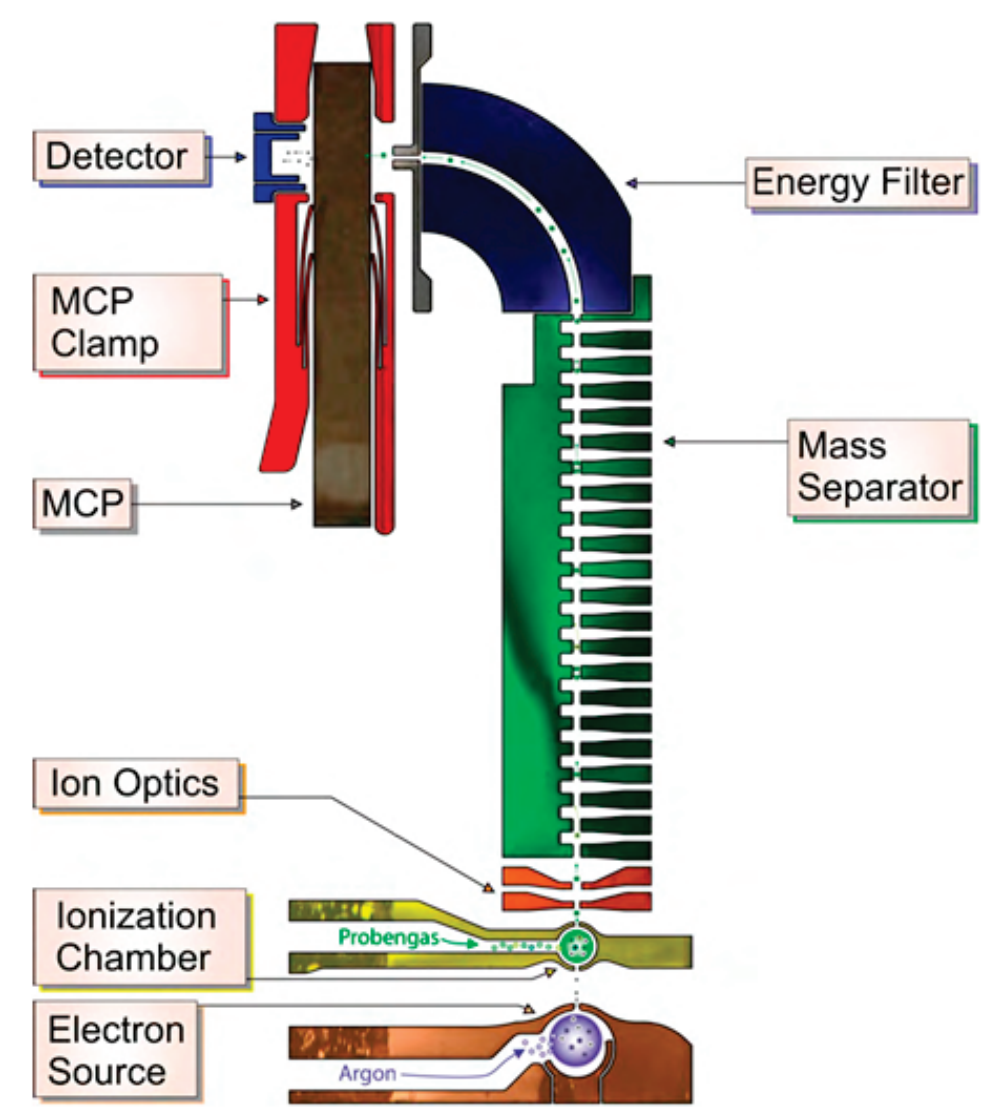

Figure 1.3: Schematic of the Planar Integrated Micro Mass Spectrometer (PIMMS) developed by Prof. Müller's group at the TUHH [2]. The PIMMS systems are batch fabricated using MEMS technologies and have a total area of about $1 \mathrm{~cm}^{2}$.

leading to a great variety of FAIMS prototypes. However, each group's approach has positive and negative points. In this section, the most relevant results for this study are briefly presented.

FAIMS uses a signal of high and low voltage pulses to filter the injected ions. The ideal waveform should be rectangular to maximize the resolution. However, producing ideal rectangular waveforms at high voltages and frequencies requires very complex circuitry. Therefore, nonideal waveforms, such as double harmonic or sinusoidal, have been successfully implemented in FAIMS measurements. Non-ideal waveforms require less complex circuitry but at a cost of a reduction in the measurements resolution $[26,6,27,7]$.

Many ion filter geometries have been proposed, e.g. cylindrical, planar, cubic, dome. Nevertheless, the most common and successful configurations are the planar (p-FAIMS) and the cylindrical (c-FAIMS), also called coaxial. The p-FAIMS (Figure 1.4) is faster, has a better resolution and can separate all kinds of ions, which makes it more suitable for standalone measurements. In contrast, c-FAIMS is more suitable for tandem mass spectrometry due to its higher ion transmission efficiency [23, 24, 28, 29]. 


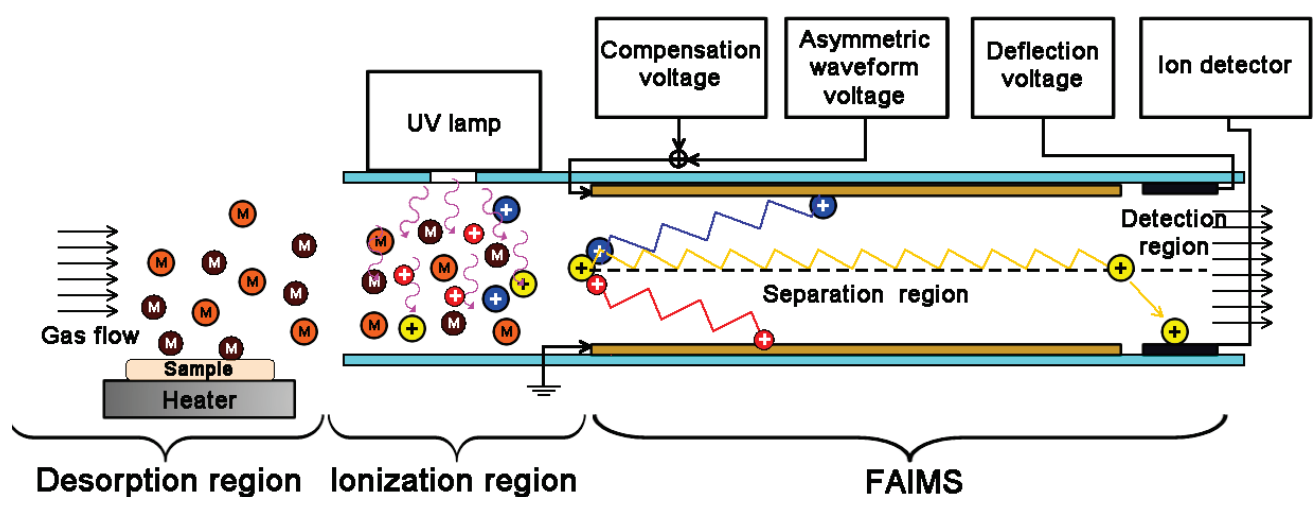

Figure 1.4: Schematic of the Planar Field Asymmetric Ion Mobility Spectrometer ( $p$-FAIMS) depicting its four main subsections: desorption region, ionization region, ion filter and ion detector.

The LoD in FAIMS depends on many factors, for example the measurement conditions, type of analyte, calculation method, detection time and more, and it is therefore not possible to define a precise general value as for the other methods discussed above. Many publications on FAIMS give vague information, such as "Detection levels below part per billion", or very wide ranges, e.g. "LoD in the ppt - ppb range". Nonetheless, most papers state a LoD of around hundreds of ppt for standalone FAIMS [23, 24, 30,31]. There were no studies found regarding the minimum amount of analyte required for FAIMS detection or the information required to deduce it from the given concentration LoD.

Acetone is a common VOC in different research laboratories and industrial facilities. Also, it is among the markers that can help detecting respiratory diseases in breath analysis studies [33]. Therefore, it is an interesting compound for the FAIMS characterization. The acetone's limit of detection in FAIMS is in the ppb levels, according to C. Ireland [31]. Prof. Kong's group at the Chinese Academy of Science gives a more precise value of $100 \mathrm{ng} / \mathrm{L}$ ( 2 ppb) [32]. Also, Owlstone Ltd. reports an acetone LoD for their FAIMS systems of $1.5 \mathrm{ppb}$ [33].

\subsubsection{Tandem Ion-mobility spectrometry-mass spectrometry (IMS-MS)}

IMS-MS separate the ions first according to their mobilities, using IMS, and then according to their mass-to-charge ratio, using MS. The IMS device is used as a pre-filter stage for the MS measurements rather than a detection system. This improves the total resolution and the signal to noise ratio, allowing the identification of a wider range of compounds and the analysis of complex mixtures.

Adding a filtering stage at the input of the MS highly increases the ion loses and, therefore, reduces the system's sensitivity. Most of the ions are lost due to the IMS filtering action and in the IMS-MS interface [23]. The LoD in FAIMS-MS is usually between tens to hundreds of ppt $[23,34,35,36]$. Finally, using a MS device adds all the previously mentioned drawbacks regarding size, cost, vacuum and other medium requirements. 


\subsection{Motivation}

High Field Asymmetric Waveform Ion Mobility Spectrometry (FAIMS) is a promising new technology for chemical identification. Reduced size and weight, low costs and the possibility of working under atmospheric conditions are among its main advantages in comparison to the existing Mass-spectrometry (MS) systems. However, in general, the sensitivity in FAIMS is lower than of state of the art MS systems. Therefore, the main objective of this project is to study the factors affecting the sensitivity in FAIMS and determine the best approach to improve it. Decreasing the system limits of detection will expand the application fields for FAIMS technology. A very promising new application would be the union of a high sensitivity FAIMS with an AFM+desorbing system. This would allow the simultaneous topographical and chemical imaging of organic samples with $\mu \mathrm{m}$ and $\mathrm{nm}$ resolution. Also, improving the FAIMS sensitivity will improve its performance in the current application fields, such as explosives and chemical weapons detection or identification of respiratory diseases by breath analysis.

\subsection{Thesis organization}

This thesis is divided into 5 chapters addressing the theoretical and experimental aspects of the topic. In Chapter 2, an overview of the FAIMS operation physical principles and mathematical models is presented. Also, the customized ion mobility simulation software is described and the simulation and experimental results are compared. In Chapter 3, the developed experimental setup is introduced and all the fabricated FAIMS prototypes and the peripheral systems are described in detail. Chapter 4 focuses on the experimental study of the parameters affecting the FAIMS sensitivity and resolution. This study is divided in different sections, each section focusing on the experimental performance of the FAIMS region, i.e. ionization, ion filter and detection regions. In the last section of this chapter, the limit of detection (LoD) of the developed system is analyzed based on the previous results. Finally, a general conclusion of the project and outlook for future work are given in Chapter 5. 


\section{Ion Mobility: Theory and Simulation}

Field Asymmetric Ion Mobility Spectrometry (FAIMS) is an analytical technique that uses high electric fields to identify ionized molecules based on their mobility. For any FAIMS study, it is essential to introduce the principles of ion generation, ion mobility in a neutral carrier gas and the other phenomena present in IMS. Therefore, this chapter provides an overview of the FAIMS operation physical principles and mathematical models outlined in literature. Finally, an ion mobility simulation software, developed from the studied theoretical model, is presented.

\subsection{UV Photoionization}

UV ionization is a very selective direct ionization method that uses photons (hv) to ionize sample molecules $(\mathrm{M})$ with ionization potential lower than the photon energy:

$$
M+h v=M^{+}+e^{-}
$$

The photon energy depends on the lamp's interior gas and the crystal of the transmission window. Common photoionization lamps emit photons with energies between 8.4 and 11.8 $\mathrm{eV}$. These photon energies allow ionization of most VOC without ionizing the carrier gas components [1], typically nitrogen (14.5 eV), oxygen (13.6 eV), hydrogen (13.6 eV), water vapor (12.6 aV), etc [37].

The ionization efficiency depends on many factors, such as the light intensity, the analyte number density and ionization potential, the ionization cross-section and the average time the analyte molecules spend in the ionization region. The light intensity in the ionization region is affected by the media's composition and the distance from the source. The Beer-Lambert law describes the light attenuation based on the media's absorption cross-section $(\sigma)$ :

$$
I_{x}=I_{o} e^{-x \sigma N}
$$

where $I_{x}$ is the light intensity at $x$ distance from the source, $I_{o}$ the light intensity emanating from the source and $N$ the gas number density, e.g. $2.5 \times 10^{25}$ gas molecules per $\mathrm{m}^{3}$ in an ideal gas at room pressure. 

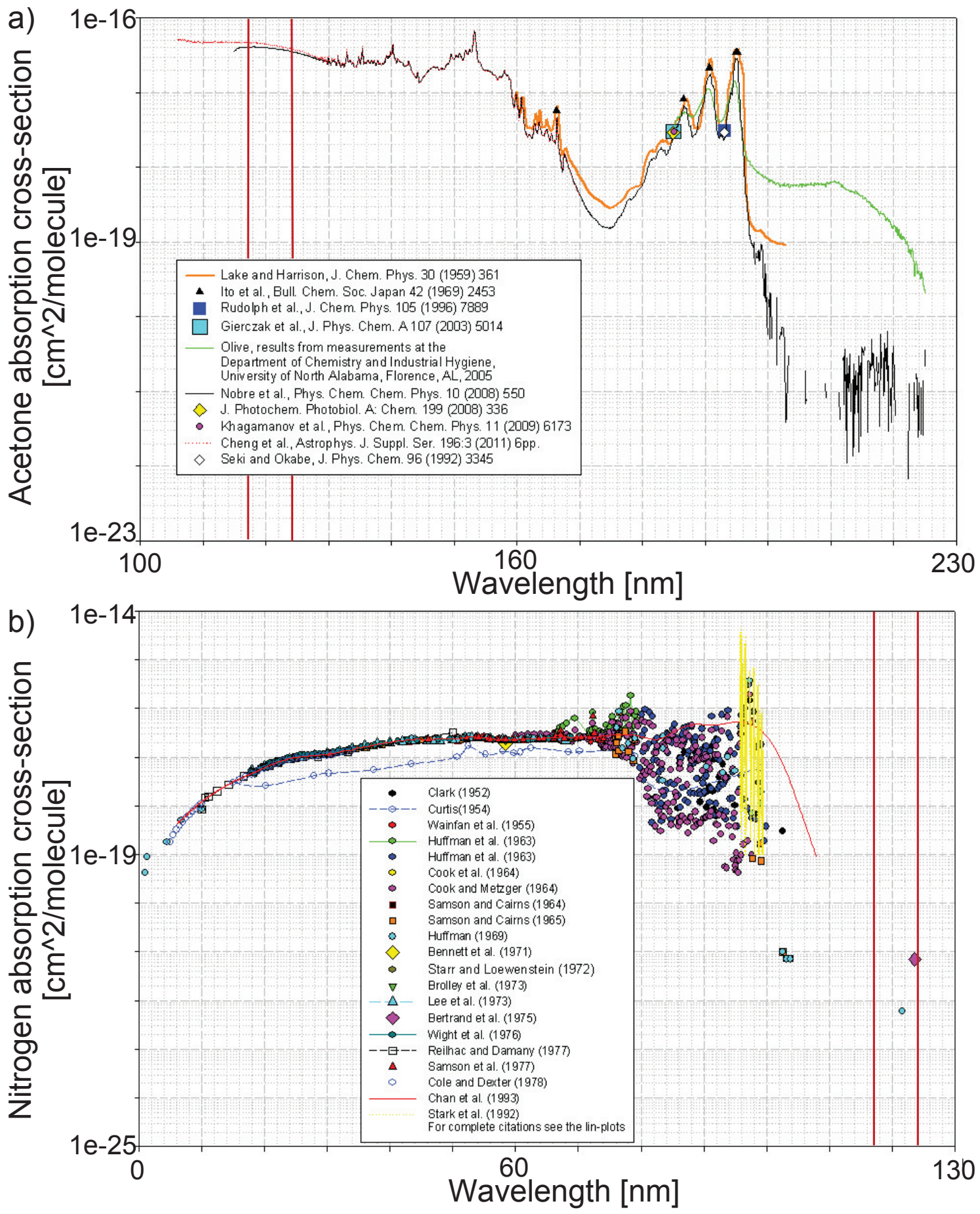

Figure 2.1: Examples of the light absorption cross sections of acetone (a) and nitrogen (b) at room temperature for different wavelengths. Plots taken from the MPI-Mainz UV/VIS Spectral Atlas of Gaseous Molecules [3]. The red lines indicate the light wavelengths emitted by the implemented UV ionization source, i.e. 117 and $124 \mathrm{~nm}$. 
In case of a media with multiple elements, the equation can be extended to take into account the effects of all the components [38]:

$$
I_{x}=I_{o} e^{-x\left(\sigma_{1} N_{1}+\sigma_{2} N_{2}\right)}
$$

The absorption cross-section depends on the incoming light's wavelength and the gas composition. Figure 2.1 shows the absorption cross-section variation at different light wavelengths for acetone (a) and nitrogen (b). Usually, the analytes have much higher absorption crosssections than the carrier gas in the UV range [3]. At low concentrations, the attenuation of the incoming light is low. Therefore, the amount of generated ions is proportional to the input of analyte molecules, i.e. a constant ratio of ions per molecules concentration. However, at higher concentrations, usually hundreds or thousands of ppm depending on the analyte, a high fraction of the incoming light is absorbed. Consequently, the produced ions versus molecules concentration ratio starts to decrease. Once the incoming light is fully absorbed, the amount of ions becomes independent of the analyte concentration $[38,39]$.

\subsection{Ion mobility dependency on the electric field}

The basic principle of IMS is the dependency of the ion's mobility on the applied electric field. When a uniform electric field is applied to a charge particle in a gaseous or liquid media, the particle is accelerated until it reaches a constant drift velocity $\left(V_{d}\right)$. The drift velocity is proportional to the magnitude of the electric field $(E)$ and the mobility coefficient $(K)$ :

$$
V_{d}=K E
$$

With weak electric fields and under standard room conditions, the ion mobility coefficient $\left(K_{o}\right)$ is constant and depends mainly on the reduced mass $(\mu)$, i.e. the effective inertial mass of the ion and the neutral molecule, and the collisional cross section $(\Omega)$ [27]. $K_{o}$ can be described by:

$$
K_{o}=\frac{3}{16} \sqrt{\frac{2 \pi}{\mu k_{B} T}} \frac{Q}{N \Omega}
$$

where $Q$ the ion charge, $T$ the drift gas temperature, $N$ the gas number density, $k_{B}$ the Boltzmann constant, $\Omega$ the ions-neutrals collision cross section and $\mu$ the reduced mass of the ion and the drift gas molecules:

$$
\begin{aligned}
& \Omega=\pi\left(r_{\text {Gas }}+r_{\text {Ion }}\right)^{2} \\
& \mu=\frac{m_{\text {Gas }} m_{\text {Ion }}}{m_{\text {Gas }}+m_{\text {Ion }}}
\end{aligned}
$$

With high electric fields to gas density ratios $(E / N)$ the ion mobility coefficient starts to variate. A commonly accepted value in IMS at which most ions have already started showing 
mobility variations is $40 \mathrm{Td}(E>1 \mathrm{MV} / \mathrm{m}$ at room conditions). However, the mobility variation threshold depends of the kind of ion, e.g. values lower than $4 \mathrm{Td}$ and higher than $60 \mathrm{Td}$ have been reported $[4,40]\left(1\right.$ Townsend $\left.(\mathrm{Td})=1 \times 10^{-21} \mathrm{~V} \cdot \mathrm{m}^{2}\right)$. Figure 2.2 shows some examples of the ion mobility dependence on the electric field $(\mathrm{V} / \mathrm{cm})$ to pressure $(\mathrm{mmHg})$ ratio for different compounds in nitrogen.

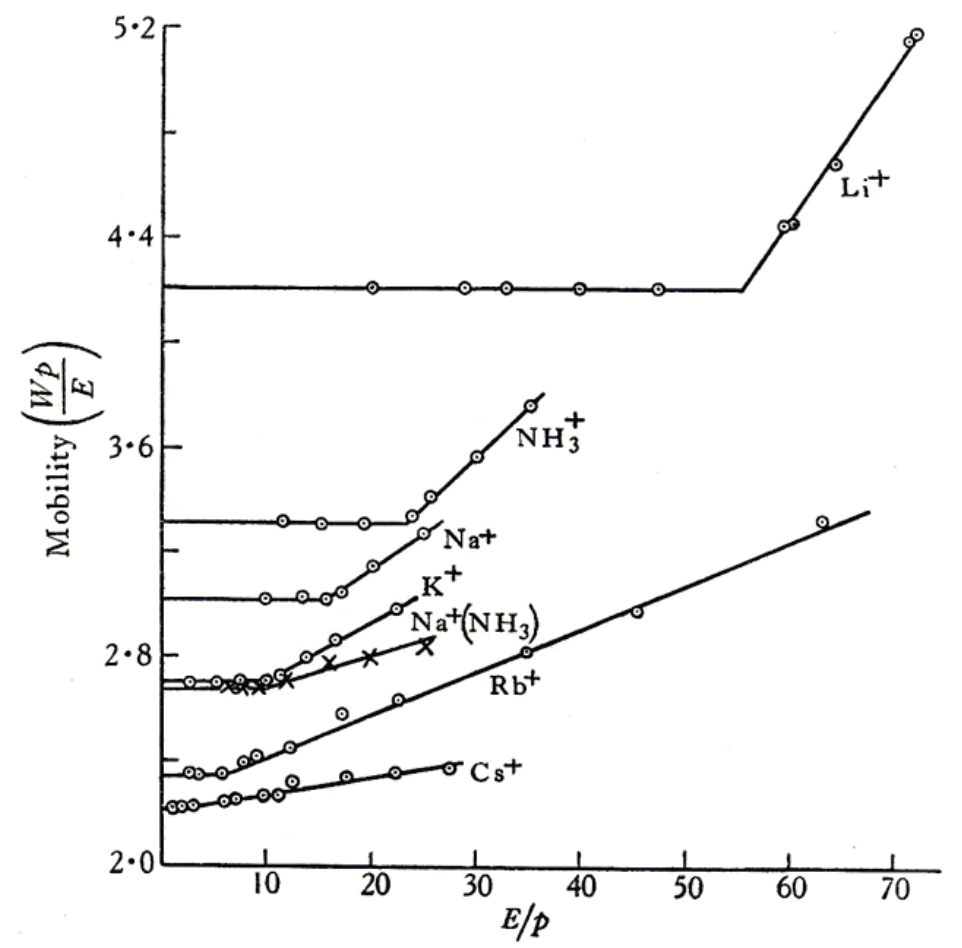

Figure 2.2: Ion mobility $v$ s E/p for different compounds in nitrogen. Image reproduced from A. Tyndall [4].

The mobility of the ions presented in Figures 2.2 and 2.3(A) always increases at high $E / p$ (or $E / N$ ), where $p$ is the pressure of the carrier gas. However, for some ions, the mobility can also oscillate or decrease, as shown in Figure 2.3(B) and (C) respectively. Different theories have been proposed to explain this behavior [23, 24, 25, 27, 5]. Nevertheless, the debate is still ongoing and there is not a universally accepted explanation for these phenomena.

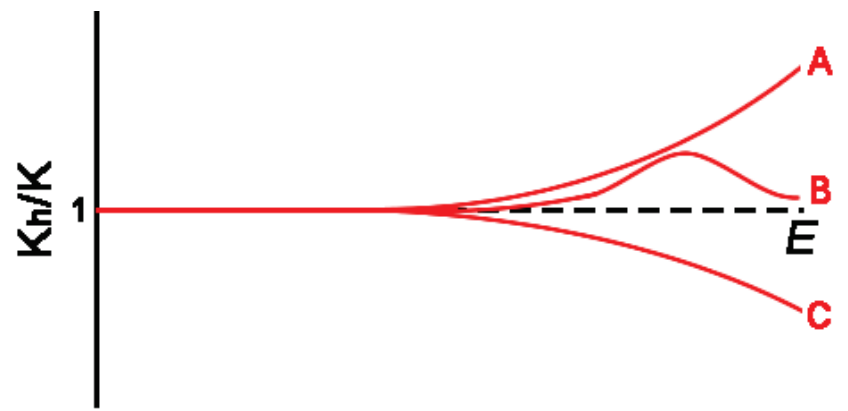

Figure 2.3: Ion mobility ratio for three characteristic ion types. 
Two of the most common explanations are the rigid-sphere model and the clustering/declustering effect: The rigid-sphere model states that, at high $E / N$ values, the force driving the ions towards the neutrals is stronger, which increases the ion-neutral collision frequency. A higher collision frequency leads to a decrease in the average ion velocity and therefore a reduction in the mobility coefficient [5].

The rigid-sphere model successfully explains the reduction in mobility but doesn't address the increasing and oscillating mobility cases. In order to explain all three types of mobility variation, a clustering/de-clustering effect has been suggested: At room conditions and weak electric fields, the ions tend to form clusters with surrounding polar molecules, e.g. water molecules. At high electric fields, the ions kinetic energy rises, imparting more energy between collisions. This leads to an increase in the local temperature of the ions, also called the effective temperature $\left(\mathrm{T}_{\text {eff }}\right)$. Higher effective temperatures modify the ion clusters either by increasing or decreasing their size[27]. The cluster size variation changes the collisional cross section and the reduced mass parameters and, therefore, its mobility coefficient [25, 5]. In order to include these effects into the mobility coefficient model, equation 2.5 has to be modified to include the $\mathrm{T}_{\text {eff }}$ variation at high electric fields:

$$
K_{o}=\frac{3}{16} \sqrt{\frac{2 \pi}{\mu k_{B} T_{\text {eff }}}} \frac{Q}{N \Omega T_{\text {eff }}}
$$

\subsection{Ion filtering}

The ion filtering principle used in FAIMS is based on the ion mobility dependency on the electric field. The FAIMS ion filter is formed by two parallel electrodes connected to a variable HV source. The ions are carried through the filtering region by a neutral gas flow. The electric field between the ion separator electrodes is created by a combination of short duration high voltage pulses and long duration low voltage pulses with opposite polarities, in such a way that the field-time products $E_{H} t_{H}$ and $E_{l} t_{l}$ are equal in magnitude. The distance traveled by a given ion perpendicular to the filter electrodes is given by: $d_{h i g h}=K E_{H} t_{H}$ for high electric fields and $d_{\text {low }}=K_{o} E_{l} t_{l}$ for low electric fields. Consequently, if Ko and $\mathrm{K}$ are the same, $\mathrm{d}_{\text {High }}$ and $d_{\text {low }}$ will be equal and the ion will return to its original position between the electrodes. However, if the coefficients are not identical there will be a shift $(\Delta \mathrm{d})$ in the ion position perpendicular to the electrodes, as represented in Figure 2.4.

The position shift $(\Delta \mathrm{d})$ accumulates after every sequence of pulses until the ions are ejected from the ion channel, while non-shifted ions remain in the channel until the detection region (Figure 2.5). The position shift can be compensated applying an extra voltage, named the compensation voltage (CV), to the filter. Since the ion mobility variation is different for any kind of ions, the compensation voltage can be tuned to keep the ions of interest in the track to the charge detector while filtering the unwanted ones. Usually, FAIMS devices implements voltage ramps as CV to scan a wide range of ions [14, 15]. 


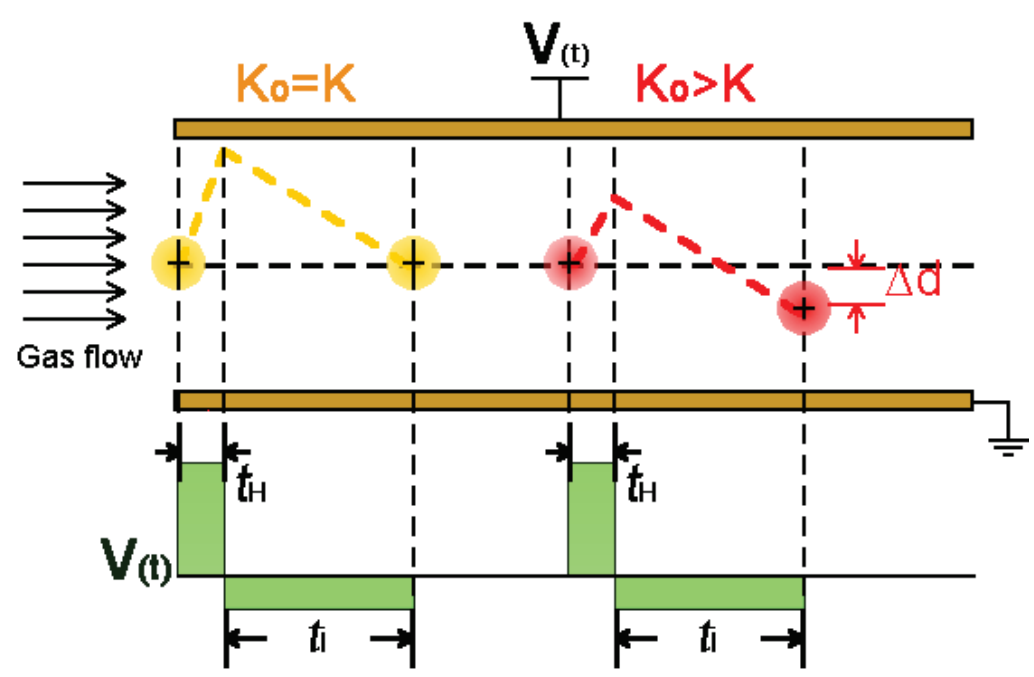

Figure 2.4: Representation of the ion trajectories of two different ions under high and low electric fields with opposite polarities. In the first case (yellow) the ion mobility doesn't change, thus the ion returns to its initial position between the electrodes. In the second case (red) the ion mobility decreases at high electric field, therefore, the ion position between the electros is shifted by $\Delta d$.

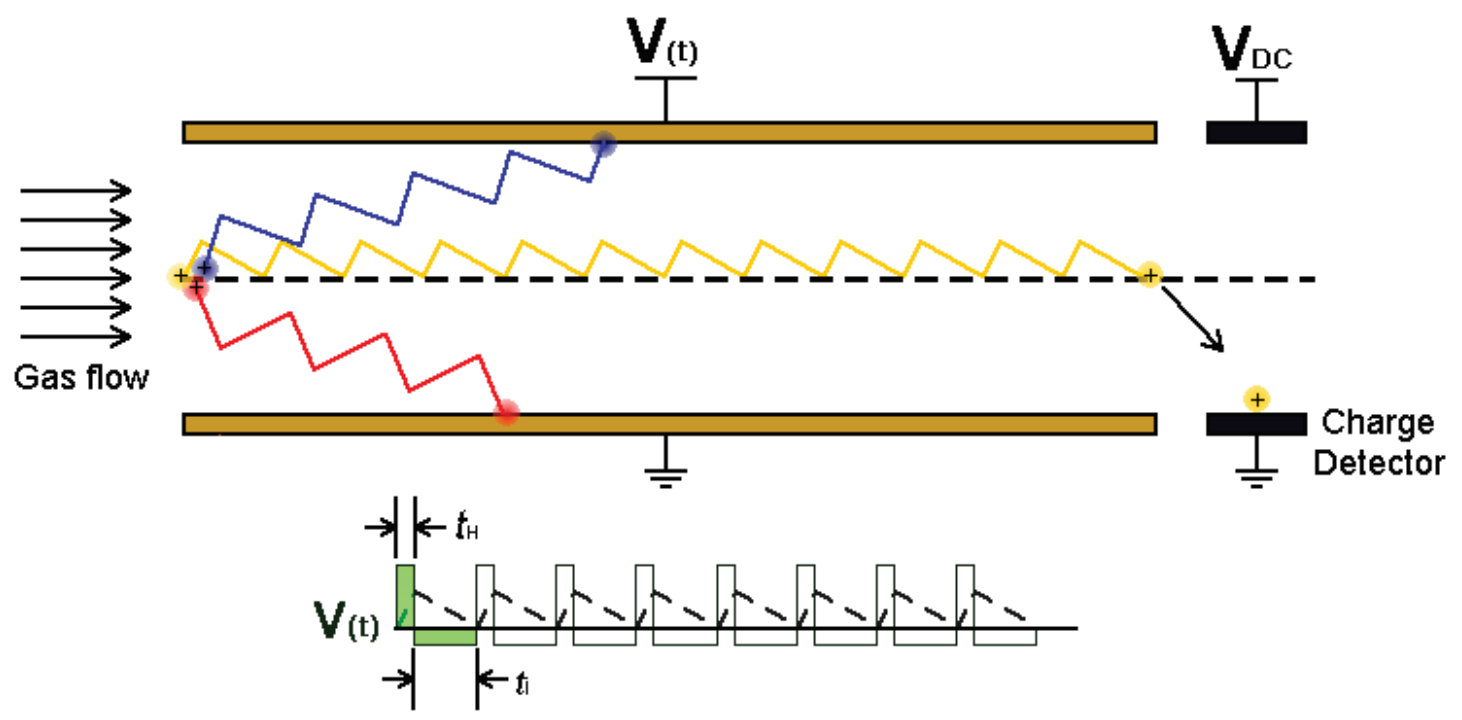

Figure 2.5: Representation of the FAIMS ion filtering principle: The ions follow different trajectories depending on their mobility variation at low and high electric fields. The compensated ions (yellow) remain in the ion channel until the detection region while the non-compensated ions (red and blue) get deflected towards the electrodes.

FAIMS measurements are displayed as ion current -vs- compensation voltage plots, where each ion peak represents one kind of ion. Different ions can be identified by their required compensation voltage. Examples of FAIMS measurements of different chemicals performed by R.A. Miller at al. [5] are presented in figure 2.6. 


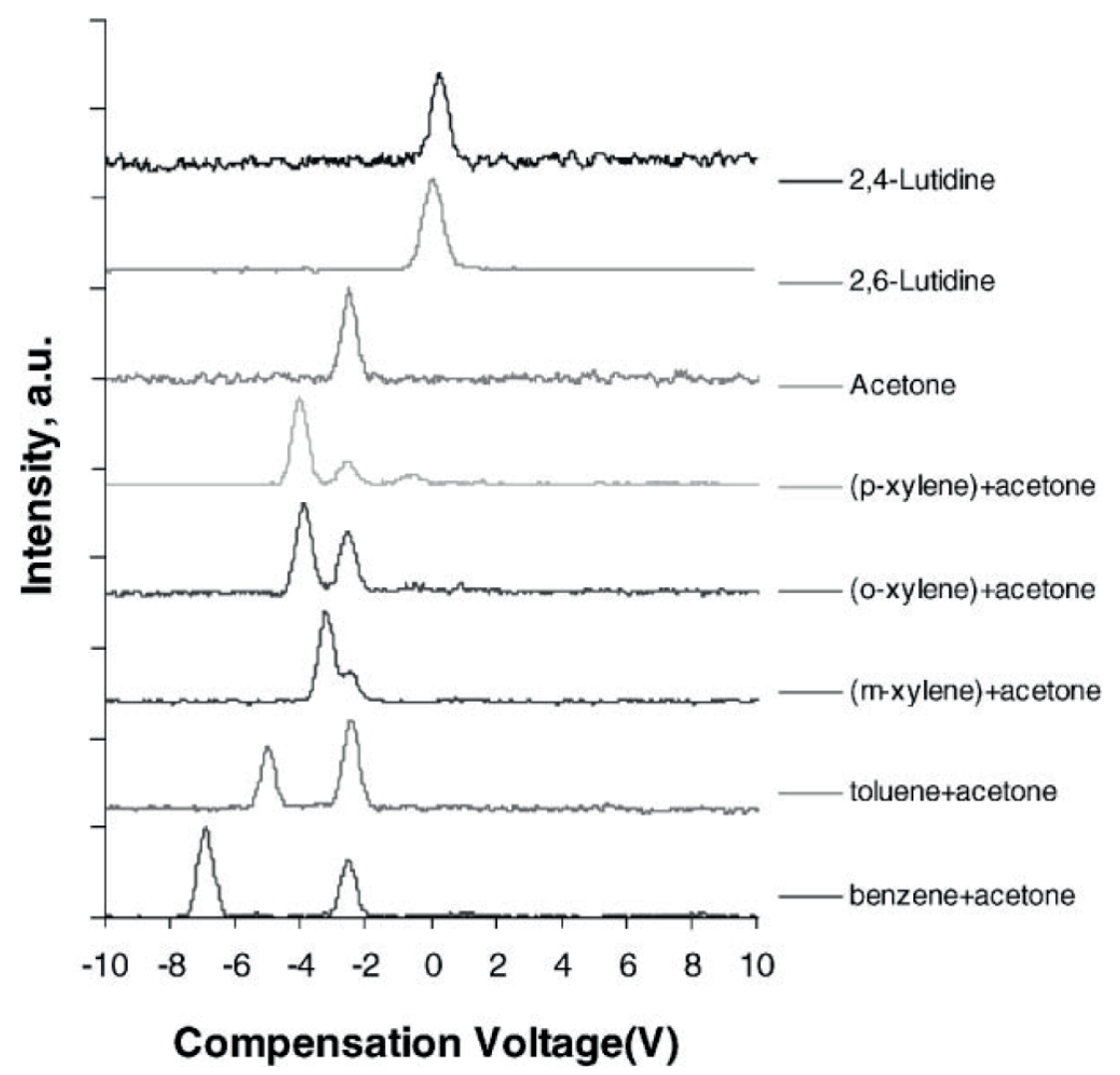

Figure 2.6: Examples of FAIMS spectra for different compounds presented by R.A. Miller at al. [5]. The ion filtering was performed at 900Vpp.

\subsubsection{Ion losses}

A key task in the improvement of the FAIMS sensitivity is the reduction of ion losses. The main ion loss mechanisms inside the FAIMS are collisions against the electrodes and recombination. Many factors can direct the ions towards the electrodes, among the most common are diffusion, Coulomb forces, electric fields or a combination of all three. The ion recombination occurs when two ions of opposite polarities or a positive ion and an electron interact with each other, neutralizing their charges. A brief description of these mechanisms is presented below.

\section{Diffusion}

Diffusion is one of the main phenomena influencing the ion's movement in a neutral media. The diffusion flow $(J)$ is mainly governed by the concentration gradient, as shown by the Fick's law of diffusion [41]:

$$
J=-D \frac{\partial \phi}{\partial x}
$$


where $\phi$ is the concentration, in amount of substance per unit volume, $x$ the position and $D$ the diffusion coefficient or diffusivity, in area per unit of time:

$$
D=\frac{3}{16} \sqrt{\frac{2 \pi k_{B} T}{\mu}} \frac{1}{N \Omega}
$$

In which $N$ is the gas number density, $k_{B}$ the Boltzmann constant, $T$ the drift gas temperature, $\Omega$ the ions-neutrals collision cross section and $\mu$ is the reduced mass of the ion and the drift gas molecules, as described in equations 2.6 and 2.7 .

Random interactions between the ions and the neutral molecules in the carrier gas can direct the ions towards the system's electrodes, neutralizing them. Therefore, diffusion has an important role in the ion losses, especially in small gap FAIMS. Diffusion losses can be diminished by reducing the ions' drift time inside the filter or by increasing the frequency of the filtering HV signal [25].

\section{Effective gap}

Ideally, the FAIMS ion filter should only neutralize unwanted ions, allowing all the ions of interest to reach the detector. However, the filter's electric field can accelerate some of the ions of interest located close to the edges of the channel towards the metallic electrodes, neutralizing them. The oscillation amplitude of the ions in the filter defines a critical area, at the edges of the ion channel, where all ions are neutralized. This reduces the effective gap $\left(g_{E f f}\right)$ where the ions can safely travel towards the detector. The effective gap width is given by [26]:

$$
g_{e f f}=g-\frac{1}{2} E_{H} K_{o} \int_{0}^{T_{W}}|f(t)| d t
$$

where $\mathrm{g}$ is the width of the physical gap, $\mathrm{K}_{\mathrm{o}}$ the ion mobility, $E_{H}$ the filtering field amplitude, $f(t)$ the filtering field normalized waveform with period $T_{W}$. The shrinking of the effective gap can be reduced by increasing the filtering frequency or decreasing the ion mobility e.g. using a different the carrier gas or increasing the pressure.

\section{Recombination}

Assuming that the anions and cations (or electrons and cations) in the ion path are evenly mixed, positive and negative particles are mutually attracted owing to Coulomb forces. When the anion-cation distance reaches a critical radius, the neutralization probability is assumed to be $100 \%$.

The particles density $\left(n_{+}, n_{-}\right)$decay due to recombination is described $[42,43]$ by:

$$
\frac{\partial n_{+}}{\partial t}=\frac{\partial n_{-}}{\partial t}=-\alpha n_{+} n_{-}
$$

where $\mathrm{n}$ is the charge density and $\alpha$ the recombination coefficient. The recombination coefficient depends on different parameters, such as pressure, temperature, relative ion-electron velocity, etc. [44, 45]. 
If the density of positive and negative particles is assumed equal $\left(n_{+}=n_{-}=n\right)$, the reduced ion density is given by:

$$
\begin{aligned}
& \frac{\partial n}{\partial t}=-\alpha n^{2} \\
& \frac{1}{n}=\frac{1}{n_{o}}+\alpha t \\
& n=\frac{n_{o}}{1+n_{o} \alpha t}
\end{aligned}
$$

where $n_{o}$ is the ions density at time $t=0$.

The ions recombination is one of the main causes of ion losses inside the FAIMS [42]. Therefore, reducing its effect will highly improve the system's sensitivity. The most viable options to reduce recombination are: reducing the ions' drift time between the ionization source and the detector or working at low pressures, i.e. reducing the amount of interactions between the ions.

\subsubsection{Alpha parameters}

The variation of the ion mobility coefficient is commonly represented by the low field mobility coefficient $\left(K_{o}\right)$, the field-mobility dependence parameters, also known as alpha parameters, and an infinite series of even powers over $E / N$ [46]:

$$
K\left(\frac{E}{N}\right)=K_{o}\left[1+\alpha_{2}\left(\frac{E}{N}\right)^{2}+\alpha_{4}\left(\frac{E}{N}\right)^{4}+\ldots+\alpha_{2 n}\left(\frac{E}{N}\right)^{2 n}\right]
$$

The alpha parameters are unique for each kind of ion. It is important to mention that there is not a physical meaning behind these parameters; they act as a convenient mathematical representation for the mobility dependence shown in equation 2.8 [6]. The number of coefficients included in the polynomial model is directly related to its accuracy. Nevertheless, high order terms are of decreasing significance and can be neglected: a forth order model is enough for a precise fitting of experimental measurements [27].

The $\alpha$ parameters are calculated based on previous experimental results and the shape of the deflection voltage signal. Several calculation techniques to determine $\alpha$ can be found in literature, one of the most commonly used methods is presented below [6, 27, 47]:

$$
\begin{aligned}
& \alpha_{2}=\frac{c_{3}}{\left\langle f^{3}\right\rangle} \\
& \alpha_{4}=\frac{c_{5}+3 c_{3} \alpha_{2}\left\langle f^{2}\right\rangle}{\left\langle f^{5}\right\rangle}
\end{aligned}
$$


where $c_{3}$ and $c_{5}$ are constant parameters that can be determined from previous measurements by:

$$
\frac{E_{C}}{N}=c_{3}\left(\frac{E_{H}}{N}\right)^{3}+c_{5}\left(\frac{E_{H}}{N}\right)^{5}
$$

$E_{C}$ is the compensation electric field corresponding to the ion of interest and $E_{H}$ deflection electric field produced by the filtering HV signal. $\left\langle f^{2}\right\rangle,\left\langle f^{3}\right\rangle$ and $\left\langle f^{5}\right\rangle$ are the separation waveform coefficients defined from the HV signal:

$$
f_{n}=\frac{1}{T_{W}} \int_{0}^{T_{W}} f^{n}(t) \mathrm{d} t
$$

where $T_{W}$ is the period of the HV waveform $(f(t))$. Some examples of the coefficients for common waveforms are shown in Figure 2.7.

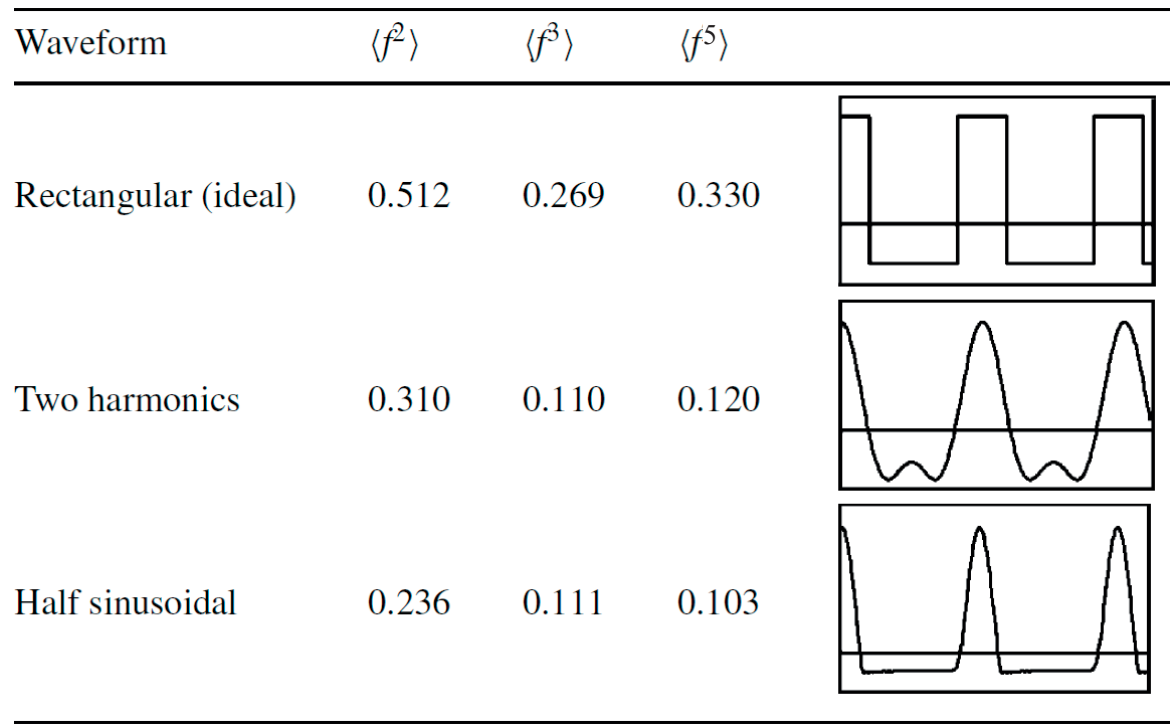

Figure 2.7: Separation waveform coefficients for common FAIMS filtering signals. Image reproduced from E. Krylov at al. [6].

\subsection{Ion mobility simulation software}

In order to have a better understanding of the ions behavior inside the FAIMS and validate the experimental results, we developed an ion mobility simulation software, in the Matlab ${ }^{\circledR}$ environment, based on the ion mobility dependency models presented in the first sections of this chapter. The software is capable of simulating the variations in the ions' trajectories inside the FAIMS caused by different electric fields and carrier gas characteristics, e.g. composition, pressure, flow rate, etc. In order to run the simulations, the user must input some parameters regarding the ions of interest, the environment characteristics as well as the geometry and electrical features of the implemented FAIMS system (including the desired CV range and step size). The software uses the user's input data and the mathematical model to calculate the ions' 
trajectories for each CV value and the expected ion current spectra. The user has the option of saving the simulated data graphically as a .jpg file or numerically as an .xls file. Figure 2.8 shows a screenshot of the software's user interface with boxes surrounding the major software components. A flowchart with the main routines included in the IMS simulation software is presented in Appendix A.

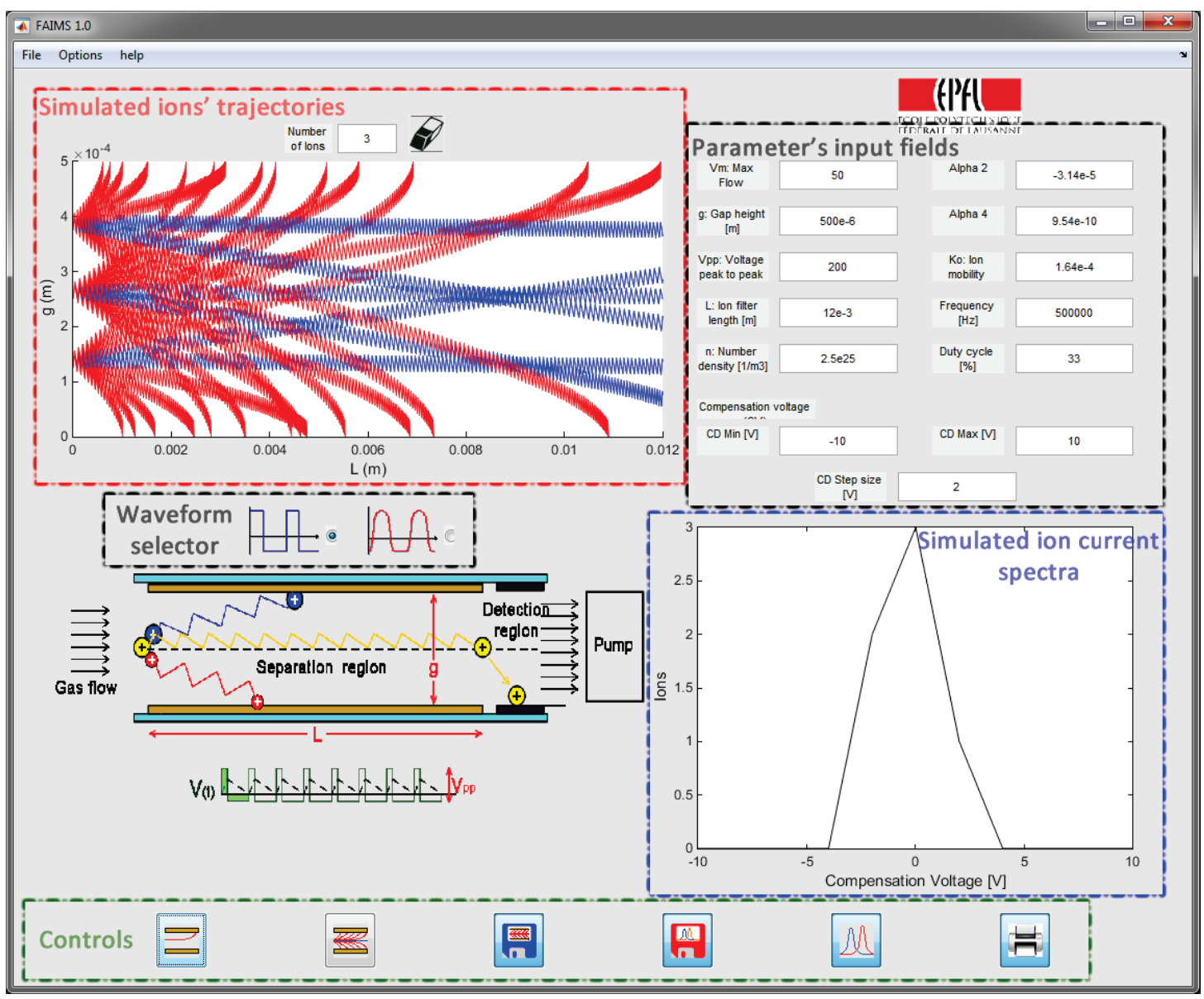

Figure 2.8: Interface window of the developed ion mobility simulation software; , the main inputloutput regions are outlined in red (Simulated ions' trajectories), black (Parameter's input fields) and blue (Simulated ion current spectra).

Another feature of the software is the possibility of simultaneously simulating the trajectories of multiple ions of the same type. The total amount of ions requested by the user are distributed equidistantly along the $\mathrm{y}$ axis at the beginning of the ions channel (at $\mathrm{x}=0$ ), as can be seen in Figure 2.8. However, the system does not process possible collisions or other interactions among the ions.

The evaluation of the simulated results was performed by comparison with experimental measurements obtained in our lab and published data from external groups. The alpha parameters for the comparison with our measurements were calculated from our experimental data while for the comparison with published data they were taken from their respective 
papers. The ion mobility coefficient $\left(K_{o}\right)$ was theoretically calculated from equation 2.5. Figure 2.9 shows the simulated and experimental ion spectra of four acetone measurements at different separation voltages. The detected and simulated ion currents are very similar up to $\mathrm{HV}=500 \mathrm{Vpp}$. The experimental ion peaks are slightly shorter $(<10 \%)$ and between $10 \%$ to $50 \%$ wider than the simulated ones. The variations in the peaks' amplitude and FWHM are due to the fact that the simulation software only considers ion losses by collision towards the electrodes, i.e. effective gap reduction, and not diffusion and recombination losses. The FWHM of the simulated peaks is determined by the ion mobility, the gas flowrate and the filter parameters, i.e. length, separation voltage amplitude and frequency.

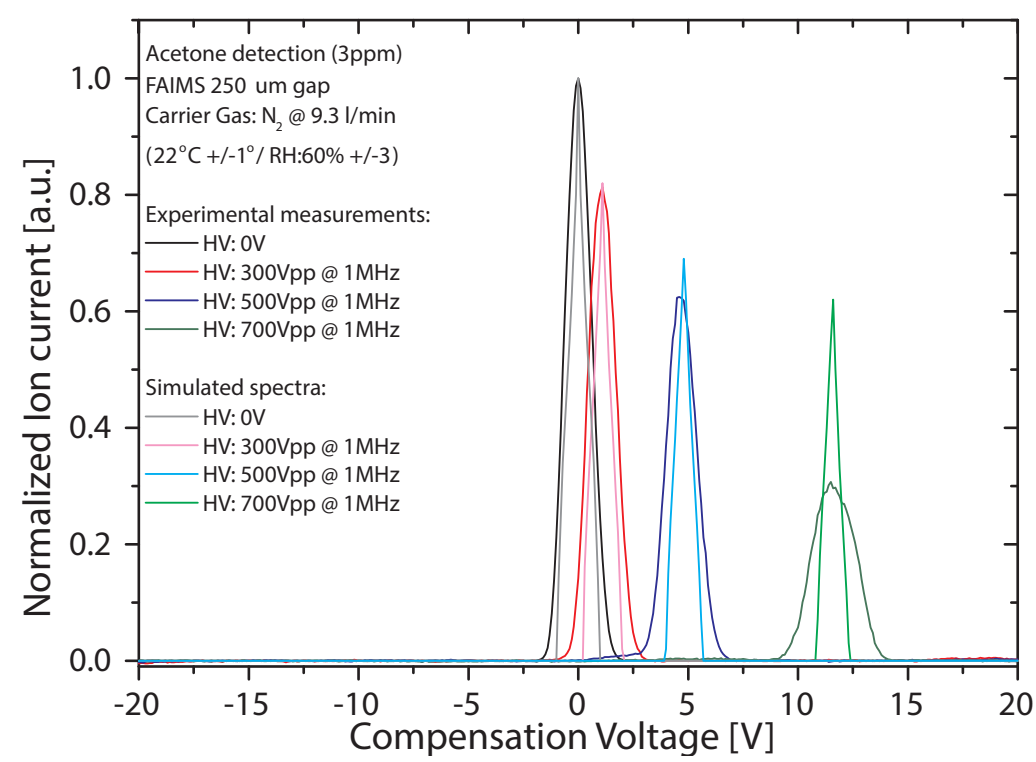

Figure 2.9: Comparison between the acetone's simulated and experimental ion spectra at different separation voltages. All measurements were performed with a $250 \mu \mathrm{m}$ gap FAIMS at a concentration of 3 ppm in a nitrogen. The carrier gas flow was 9 liters per minute and relative humidity was $60 \%$.

At $\mathrm{HV}=700 \mathrm{Vpp}$, the experimental ion peak becomes considerably wider and smaller than the simulated one. This can be explained by the separation of similar kinds of acetone ions, e.g. monomers-dimers or acetone-water clusters of different size, only observed at higher electric fields. In other words, the wider peak is the superposition of two different peaks that are just starting to shift apart. This also explains the reduction of the amplitude by two. The integrals of both the simulated and experimental peaks are the very similar, i.e. exactly half the ones of the non-filtered peaks, which agrees with the proposed explanation.

Nevertheless, the simulated ion separation and ion mobility dependency at high electric fields, which was the main motivation for the development of this software, agree well with the experimental results. Figure 2.10 presents two CV-vs.-HV plots summarizing several acetone measurements at different relative humidity $(\mathrm{RH})$. The experimental measurements in Figure 2.10.a were performed in our lab, using a FAIMS device with an ion gap of $250 \mu \mathrm{m}$. The experimental data in Figure 2.10.b was published by prof. Kong's group [7] from the Chinese 
Academy of Science (CAS), using a $500 \mu \mathrm{m}$ gap FAIMS. In both cases, the experimental results fit very well with the simulated and theoretical models.
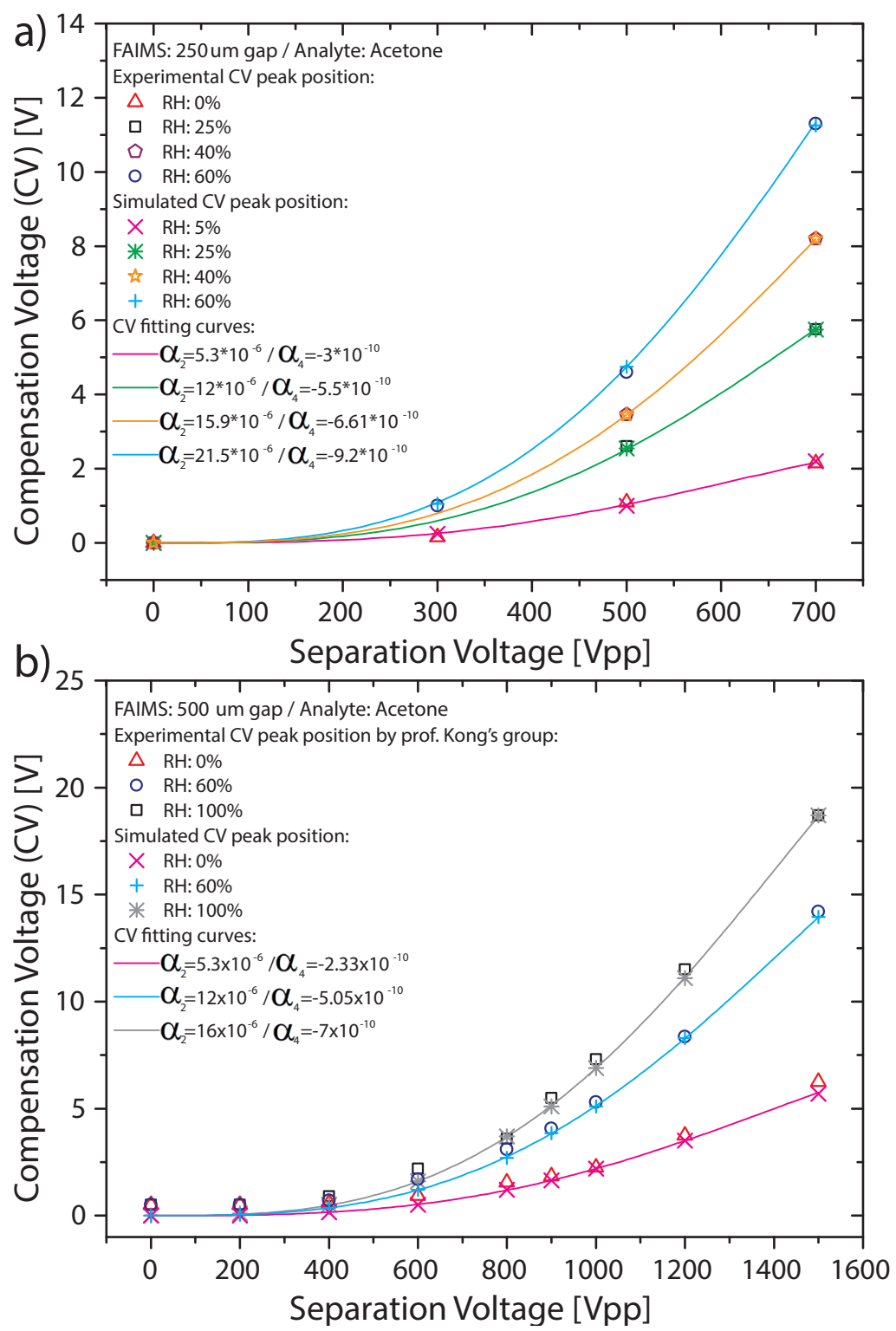

Figure 2.10: Compensation Voltage shift-vs.- separation voltage plots for acetone at different $R H$ levels. The simulated and experimental data are compared with a theoretical fitting curve. The experimental data in plot (a) was obtained in our lab while the experimental data in plot (b) was taken from an external paper [7].

\subsection{Conclusions}

In this chapter, the physical phenomena behind the UV ionization, the ion mobility separation technic and the ion losses inside the FAIMS were presented. Also, some commonly accepted mathematical models describing the ions' mobility dependency on the $\mathrm{E} / \mathrm{N}$ ratio were briefly 
introduced. These mathematical models were used to create an ion mobility simulation software. The developed software was described and several plots comparing simulated and experimental results, from internal and external sources, were shown. These comparisons exhibited high accuracy levels between simulated and real measurements. The IMS software will be very useful in predicting and corroborating future experimental results. 


\section{Design and Fabrication of the FAIMS system}

This chapter starts with an overview of the designed FAIMS system. All the manufacturing processes and materials used in the fabrication of the FAIMS devices are presented. Finally, the implemented peripheral systems are described, including pictures and schematics of the designed circuits and measurement setups.

\subsection{System's overview}

All IMS systems, including FAIMS, are formed by four basic subsystems: the sample introduction, the ionization source, the ion filter and the detection systems. These subsystems are placed sequentially through the ion path in order to perform all the required processes for chemical identification. Figure 3.1 shows the schematics of the designed FAIMS prototypes. The main difference between both approaches is the ionization technique: one of our systems uses corona discharge ionization (Figure 3.1.a) and the other UV ionization (Figure 3.1.b). The core of the FAIMS system is formed by a set of electrode plates separated by a spacer structure which forms the ion channel. The different electrodes and geometry features on the plates define the ionization, filter and ion detection regions of the FAIMS, as shown in Figures 3.1 and 3.4. Each core section is connected to one or several peripheral systems. The peripheral systems either generate or record the necessary input and output signals during the measuring process.

The first step in a FAIMS measurement is to desorb and inject the selected analytes into the system. In our case, the analytes desorption is done in a commercial V-OVG calibration gas generator from Owlstone nanotech. This device can produce stable gas flows with analyte concentrations between ppt and ppm levels, i.e. from $10^{13}$ to $10^{19}$ analyte molecules per $\mathrm{m}^{3}$. All molecules and ions are driven through the FAIMS by a carrier gas flow generated by a pump or a pressurized gas bottle. Figure 3.2 shows a schematic of the experimental setup used for the FAIMS measurements.

The next step of the FAIMS measurement is the ionization of the desorbed molecules. Two atmospheric pressure chemical ionization techniques were implemented: corona discharge and UV photo-ionization. In the first case, a high electric field is applied between a sharp electrode and a flat counter-electrode generating a small plasma region. The seed ions created by the plasma interact with the analyte molecules, thereby ionizing them. The ionization region of the corona discharge is located outside the electrode plates, as shown in Figure 3.1.a. 
In the second case, the UV ionization is performed by a $10.6 \mathrm{eV}$ krypton lamp which injects photons directly into the ion channel to ionize the analyte molecules (Figure 3.1.b).

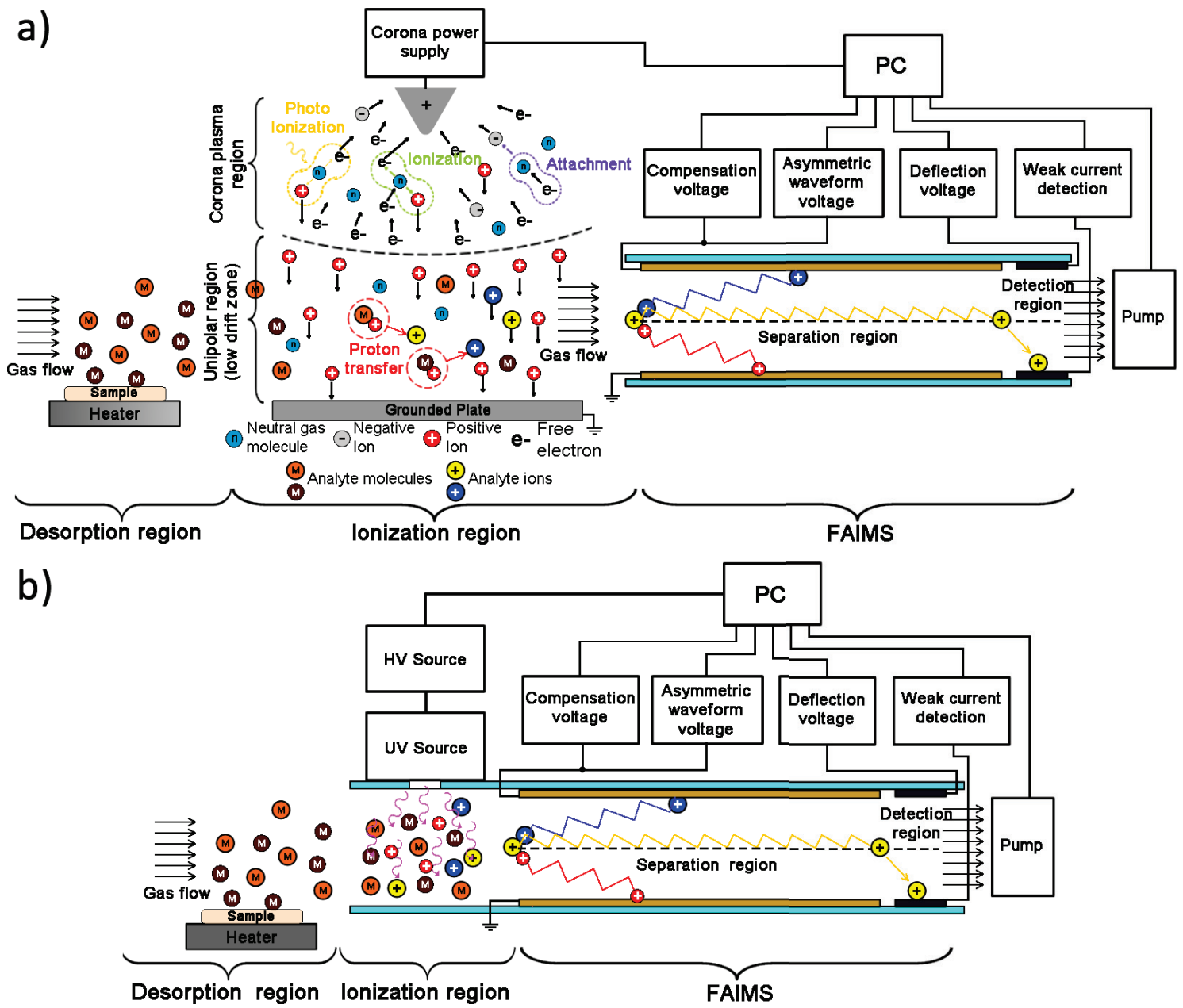

Figure 3.1: Schematics of the FAIMS including peripheral systems. Two different ionization sources are represented: (a) corona discharge and (b) UV source ionization.

After the ionization process, the produced ions are filtered according to their mobility. This filtering is performed by applying a high voltage asymmetric waveform to the electrodes in the separation region. The circuit generating the high voltage (HV) signal was designed based on the n-MOS inverter configuration using two IXTH1N200P3 n-channel power MOSFETs for the voltage switching. The circuit is capable of delivering rectangular signals with amplitudes up to $1 \mathrm{kV}$ at frequencies in the $\mathrm{MHz}$ range. A voltage ramp, named compensation voltage (CV) ramp, is superimposed to the HV signal to scan a wide spectrum of ions. A sinusoidal signal can be added to the CV ramp for lock-in detection measurements. All signals are controlled by the user and can be modified to optimize the measurements.

Finally, the ions enter the detection region. In the detection region, all ions are deflected towards the detector electrode by an electric field. The current produced by these ions is amplified by a low noise current amplifier with a gain of $\times 10^{11}$. The detected ion currents are 
recorded by a PC using a DAQ board connected to the output of the amplifier. The data is then processed and displayed by a customized LabVIEW ${ }^{\circledR}$ software.

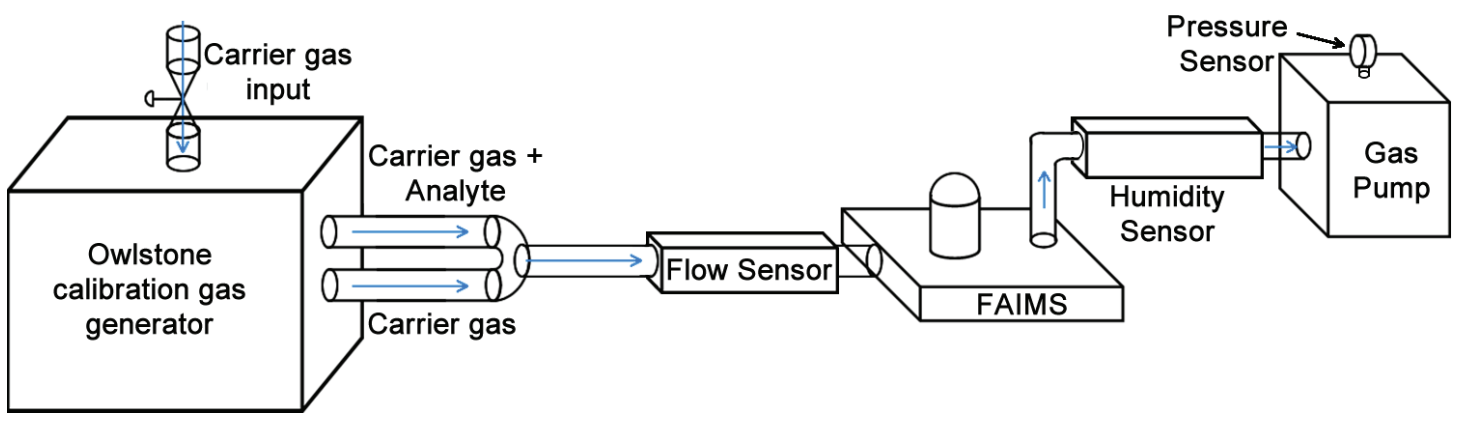

Figure 3.2: Schematic of the experimental FAIMS measurements setup. A commercial V-OVG calibration gas generator from Owlstone Ltd. is used as the analyte desorption/injection system. Different sensors are implemented to monitor the experimental conditions, e.g. gas flow, humidity, temperature, pressure.

\subsection{FAIMS core}

As mentioned before, the designed FAIMS prototype is a set of peripheral devices connected to a core. This core is formed by the electrode plates, the protection case and the electronic and carrier gas connectors. Three core prototypes have been designed and fabricated in the frame of this Ph.D. thesis with different geometries to fit the selected ionization sources and detection systems. Figure 3.3 shows the exploded view of the cores' 3D designs.

\subsubsection{Electrodes plates}

Several sets of electrodes plates were fabricated using different materials and fabrication techniques. The electrodes geometries are based on the design used by our partners in Prof. Kong's Laboratory at the CAS. The schematics of the fabricated electrode plates, including the dimensions of all their features, are included in the appendix B. The main requirement for the materials selection for the electrode plates are: good electrical conductivity for the electrodes and very high electrical insulation for the substrate, i.e. to avoid current leakages.

The first set of plates was made by screen printing the electrodes on a $1 \mathrm{~mm}$ thick Alumina substrate $\left(\mathrm{Al}_{2} \mathrm{O}_{3}\right.$ 96\%) using a highly conductive silver-palladium ink (ESL9635-HG). On the inner side of the plates, the ion filter, ion detector and grounding electrodes were printed while the other side contains the connectors for the peripheral systems. The electrodes at both sides are interconnected though the substrate by $500 \mu \mathrm{m}$ diameter vias. The printed layers have a thickness of approximately $8 \mu \mathrm{m}$ and a sheet resistance lower than $20 \mathrm{~m} \Omega / \mathrm{sq}$. 
a)

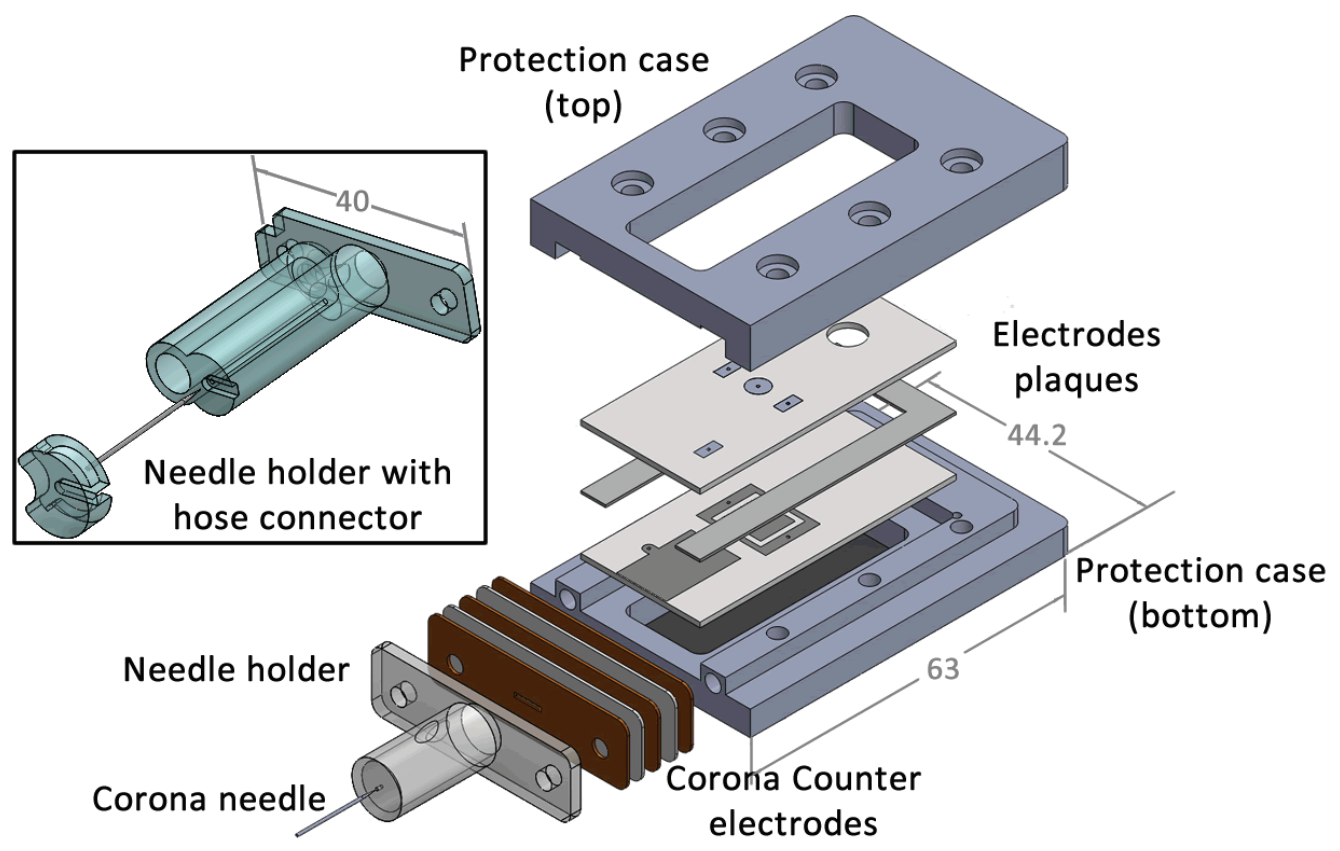

b)

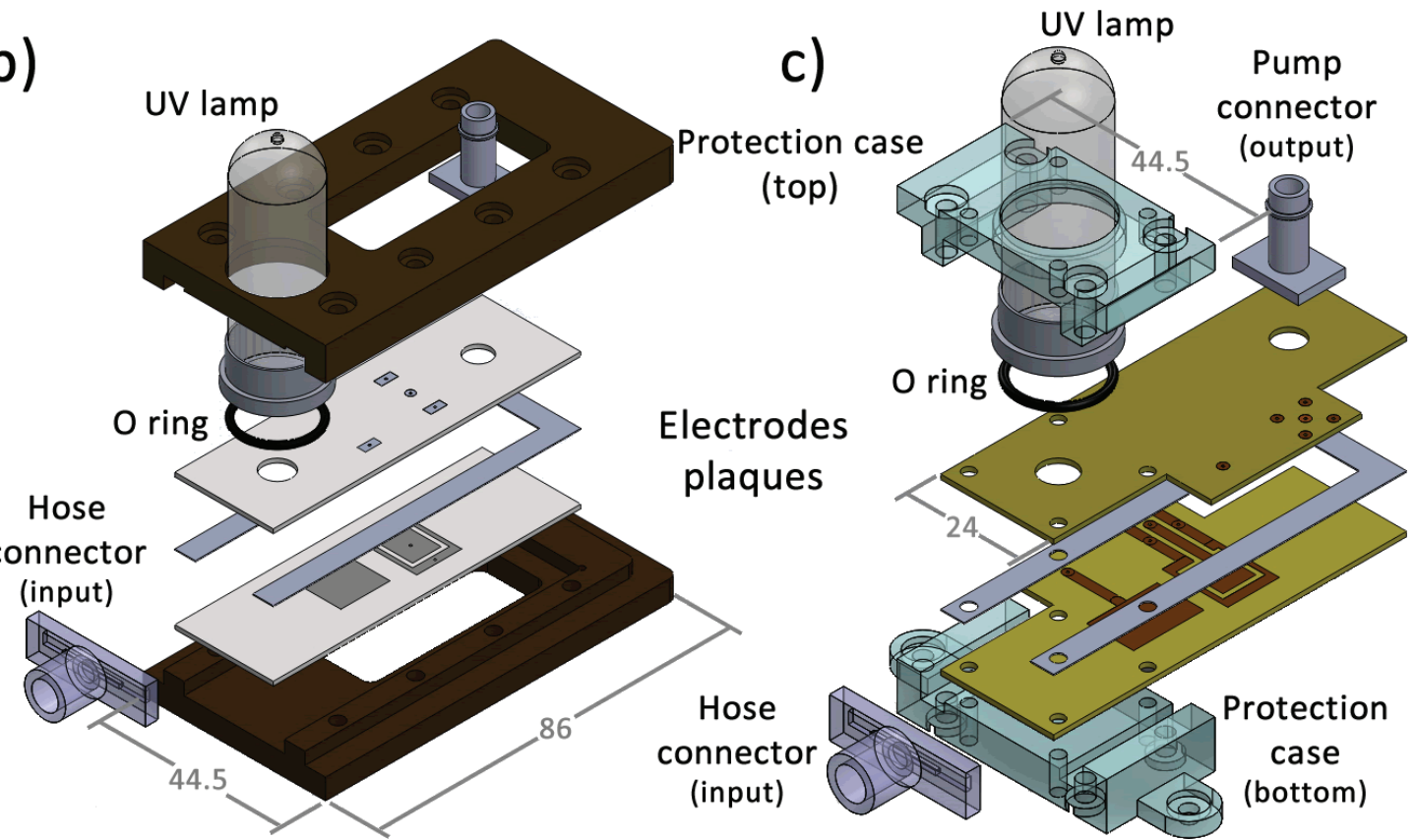

Figure 3.3: Exploded view of the three fabricated FAIMS cores (dimensions in $\mathrm{mm}$ ). The design in a) has a corona discharge ionization system while b) and c) have UV ionization sources. The electrode plates in were fabricated in alumina, (a) and (b), and in FR4, (c). Two needle holders were made in model a) with different sample input geometries.

All geometrical features in the alumina substrate were fabricated by laser cutting. The spacers for all the prototypes were also made by this technique, using alumina substrates with thickness from 170 to $500 \mu \mathrm{m}$. Figure 3.4 shows pictures of all the fabricated electrodes plates and the spacer. The length of the UV FAIMS plates is longer than the ones of the Corona FAIMS to 
include the support of the UV lamp.

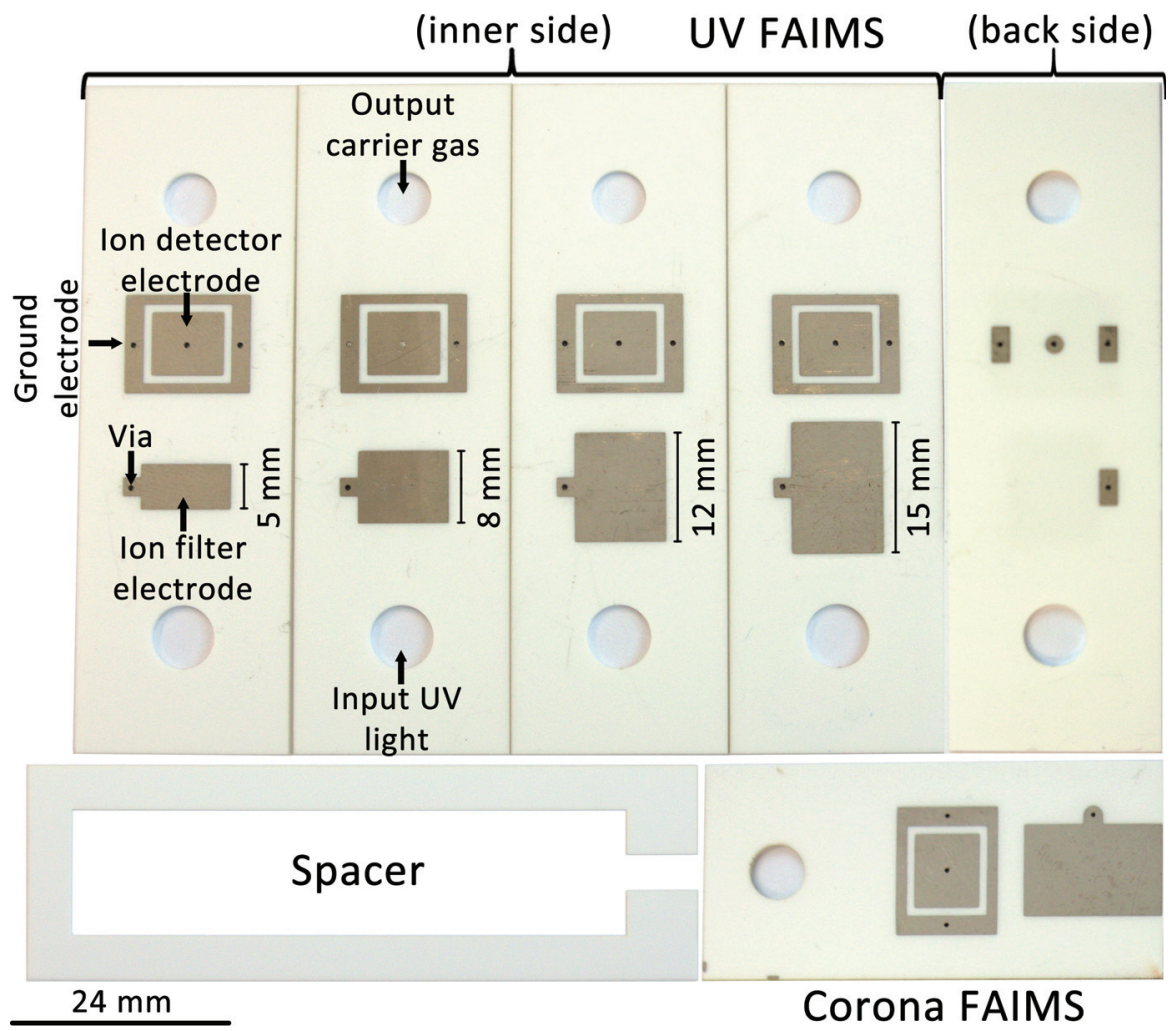

Figure 3.4: Fabricated electrode plates with alumina substrate. The electrodes are screen printed on the substrate using silver-palladium ink.

In order to simplify the fabrication processes and reduce production times and costs, a second set of electrodes plates was fabricated using PCB prototyping techniques on FR4 substrates. Thin copper layers (12-35 $\mu \mathrm{m}$ ) were laminated on the substrates and then etched to form the electrodes. Finally, a layer of tin is deposited on the electrodes to improve the soldering. Two electrode geometries were designed with different locations for the peripherals connector pads. The first design, shown in Figure 3.5.a, has a similar geometry to the fabricated in alumina, with vias connecting the electrodes in the inner face to the connection pads on the backside. This design reduces the distance between the electrodes and the pads, decreasing the effect of external factors in the detected signal. However, it requires depositing conductive layers on both sides of the substrate and extra fabrication processes to create and metalize the vias. Also, the peripheral connectors should be of the surface-mount type, which requires the use of conductive glues since some of the electrodes are not easily accessible. These kinds of glues have higher electrical resistance than traditional solder materials.

In the second design, the connection pads are placed outside the ion channel on the same side of the electrodes (Figure 3.5.b). This provides better access to the connection pads and allows the use of through-hole connectors. The through-hole connectors are more mechanically stable than the surface mounted ones and can be soldered to the pads which improve the electric resistance. Another advantage of this design is that it only requires one conductive 
layer and no vias, which reduces the fabrication time and costs. Nevertheless, the use of through-hole connectors might increase the amount of noise in the detected signals.

An extra set of electrodes plates was designed for the analysis of ion losses inside the FAIMS system (Figure 3.5.c). It is formed by an array of electrodes sequentially placed through the ion channel's length. Once the plates are set in place, each electrode faces a counter electrode, forming several ion deflection/ detection systems at different distances from the UV source.

a)

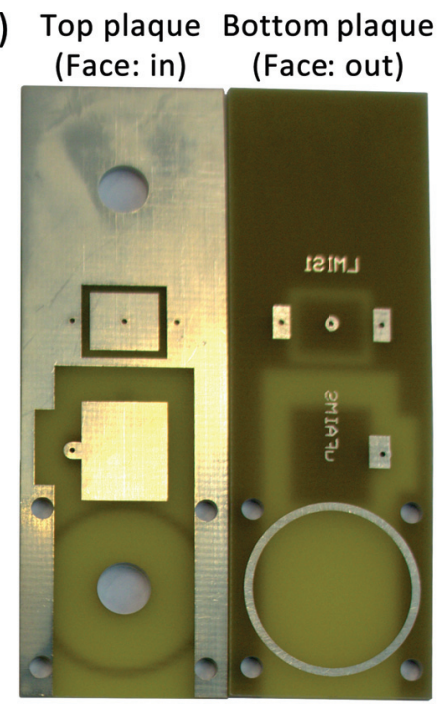

b) Top plaque Bottom plaque
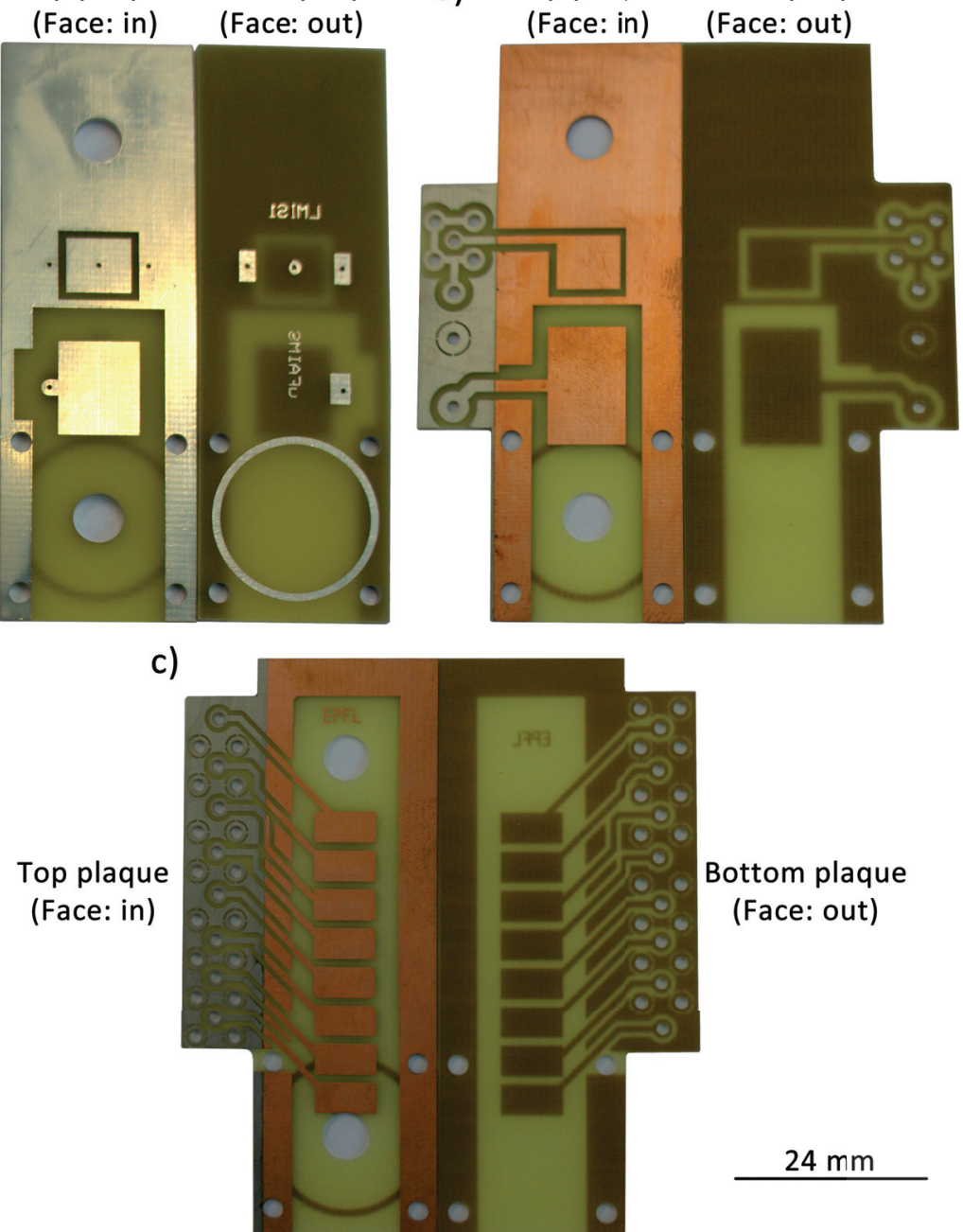

Figure 3.5: Fabricated PCB electrode plates for FAIMS $(a, b)$ and ion losses measurements (c). The plates in (a) have a similar geometry to those fabricated in alumina, with vias connecting the electrodes in the inner face to the connection pads on the backside. The plates in (b) and (c) have conductive layer only on the inner face and the connection pads are placed at the same side of the electrodes to simplify the connection. Also, (b) and (c) only have the tin layer on the connection area, for the electrodes section the copper layer was left uncovered. 


\subsubsection{Assembly of the FAIMS core}

The fabricated electrode plates and spacer are glued together using nonconductive epoxy glue (LOCTITE EA 9483). Special care was taken to ensure that the electrodes face their respective counter electrodes as accurately as possible. Then, all the connectors are glued to the plates using the LOCTITE EA 9483 epoxy glue for the nonconductive parts and the 8330S Silver Conductive Epoxy adhesive for the electrically conductive ones. Both glues are cured at $150{ }^{\circ} \mathrm{C}$ for five minutes. Finally, all the cables are soldered to the connection pads using tin. Examples of the fully assembled alumina and PCB FAIMS cores are presented in Figure 3.6.

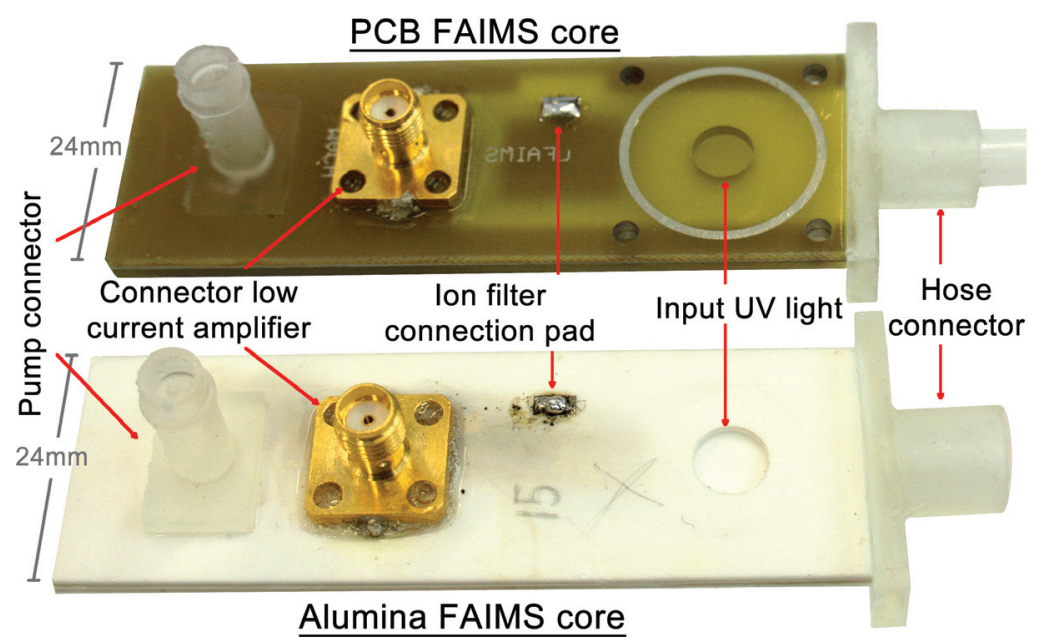

Figure 3.6: Assembled FAIMS cores using the fabricated PCB and alumina electrode plates.

\subsubsection{FAIMS core prototypes comparison}

The four fabricated core prototypes were tested using the experimental setup in Figure 3.2. All core systems worked satisfactorily, allowing filtering and detecting different types of ions. The alumina and PCB cores, with the same geometry, showed very similar results. Therefore, using PCB electrode plates is a good approach to reduce the fabrication complexity and costs without affecting the system's behavior. Finally, the designs using "surface mounted" connectors at the opposite face of the plates (Figures 3.4 and 3.5.a) were less vulnerable to external noise than the designs with "through hole" connectors on the same face as the electrodes (Figure 3.5.b).

\subsection{Peripheral systems}

\subsubsection{Ionization source}

Two ion sources were implemented: one UV ionization source and one corona discharge ionization source. Both ionization sources use a $5 \mathrm{kV}$ Wallis Photomultiplier Supply as voltage source. The selected UV lamp is the Heraeus PKS106. This lamp ignites at - $1500 \mathrm{~V}_{\mathrm{DC}}$, producing a light beam with ionization photon energies of 10.0 and $10.6 \mathrm{eV}$, i.e. 124 to $117 \mathrm{~nm}$ wavelengths. The lamp is kept in place on the FAIMS core by the CNC fabricated protection case, as shown in Figure 3.7. An O-ring is placed between the lamp and the electrodes plate to avoid gas leaks. 


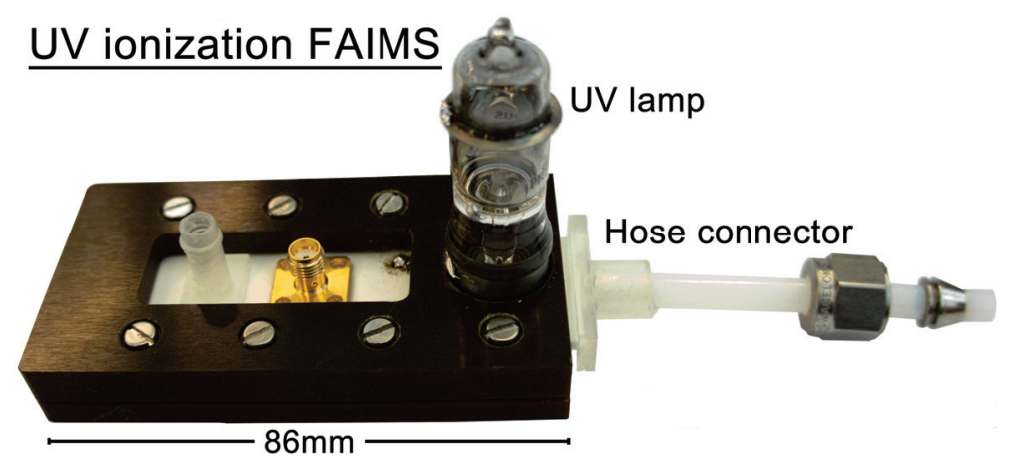

Figure 3.7: FAIMS system including the protection case and the UV source.

As shown in Figure 3.3.a, the fabricated corona discharge ionization source has a pin-plate configuration. A tungsten-carbide needle with a point radius of $7 \mu \mathrm{m}$ was selected as the anode and a $0.5 \mathrm{~mm}$ thick copper plate functioned as the cathode. The corona discharge occurs at voltages between 2000 and $2500 \mathrm{~V}_{\mathrm{DC}}$ for an anode-cathode distance of approximately $5 \mathrm{~mm}$. The needle holder, which also works as hose connector, was fabricated using a 1+FormLabs 3D printer. Figure 3.8 shows a picture of the fully assembled corona FAIMS system.

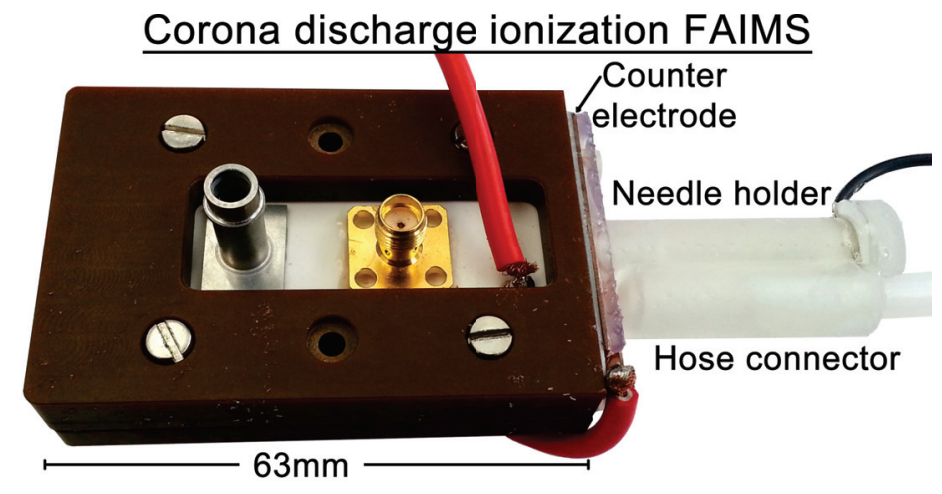

Figure 3.8: FAIMS system with the protection case and the corona discharge ionization source.

The experimental measurements performed with both ionization techniques showed similar ionization efficiencies. However, the corona discharge system must be carefully tuned to avoid generating high levels of electrical noise which can affect the FAIMS measurements. Also, the corona's tip electrode rapidly loses its sharpness, which might lead to variations in the ionization behavior. Therefore, the rest of the thesis will present data obtained with the UV ionization system.

\subsubsection{Ion filter electronics}

Filtering ions in FAIMS requires rectangular voltage signals with amplitudes of hundreds or even thousands volts at frequencies in the $\mathrm{MHz}$ range. The most challenging part of fabricating a circuit capable of producing such signals is to find a very fast power switching device. One of the simplest approaches to generate rectangular waveforms is the CMOS configuration. Unfortunately, no commercially available pMOS transistors were found that were able to work 
at the required voltage levels. Consequently, the CMOS approach had to be replaced for a customized nMOS inverter configuration. A schematic of the fabricated circuit is presented in Figure 3.9. This circuit is capable of generating square voltage signals up to $1000 \mathrm{~V}_{\mathrm{PP}}$ at frequencies in the $\mathrm{MHz}$ range.

Since in the designed circuit the pMOS transistor is replaced by a second nMOS transistor, two opposite signals are required to perform the voltage switching. Therefore, an initial digital stage was added to split the control signal in two identical signals. Then, one of the split signals is inverted using a 74AHC06 HEX inverter. In order to avoid phase shifts between the signals, both inverted and non-inverted signals are passed through the 74AHC06 integrated circuit. Additionally, a short delay had to be added between the transistors ON and OFF transitions to avoid simultaneous conduction and, therefore, a short-circuit. With this aim, a small delay system was added to each of the control signal branches. This system was designed to add an $\sim 80$ ns delay to the signal's OFF transition by the discharge of a $\sim 15 \mathrm{pF}$ capacitor through a 6.8 $\mathrm{k} \Omega$ resistor. By placing a $1 \mathrm{~N} 4148$ diode in parallel to the $6.8 \mathrm{k} \Omega$ resistor, the delay is avoided during the $\mathrm{ON}$ transition. This delay can be modified by changing the value of the discharge resistor. The control signals' variations at different points of the initial inverter stage are shown in the waveform diagram in Figure 3.9.

The second stage of the circuit implements two gate drive optocouplers (HCPL-3180) to isolate the digital stage, i.e. the control signal inverter, from the power stage. It also amplifies the control signal from $5 \mathrm{~V}_{\mathrm{PP}}$ to the $18 \mathrm{~V}_{\mathrm{PP}}$ required to drive the power transistors. In the power stage, two nMOS transistors switch the DC voltage from the high voltage source (EPS-3000PIIV) to generate a rectangular waveform. Since the source voltage of the upper transistor changes from 0 to $\mathrm{HV}$ when it starts conducting, a floating DC source at the gate is required to keep the $\mathrm{V}_{\mathrm{GS}}$ constant. Two $9 \mathrm{~V}$ commercial batteries were selected to provide the required floating voltage. They showed good driving capabilities without affecting the resulting signal. Different DC/DC converters, e.g. JCD0624S15, JCD0624S15H, were also tested as floating sources but their high parasitic capacitances deformed the output signal. Finally, a high-pass filter was added at the output of the nMOS inverter to remove the DC component from the high voltage waveform. This leads to the asymmetric signal required in FAIMS.

It is important to carefully select the power stage elements. Most high power devices have very high parasitic capacitances which slow down the circuit response and deform the output signal. In our case, the best observed results were obtained using IXTH1N200P3 power nMOS transistors for the voltage switching and VISHAY high precision resistors (PTF65) and capacitors (564R30GAS10) for the DC filter. Additionally, some extra elements were used to improve the system's behavior: Capacitors banks were placed at the source input of the different circuit devices to avoid power fluctuations. The power transistors were attached to two heat dissipators to avoid overheating them. Three low dropout voltage regulators (LDO) were added to convert the $\pm 20 \mathrm{~V}$ input into the voltage levels required by the other fabricated circuits, i.e. \pm 8 and +5 V. A low-pass filter was added at the circuit's output reduced the coupled noise from the high voltage signal to the ion detector. Also, the circuit was placed in a shielded box to further reduce the noise coupling. A picture of the fabricated circuit, inside the shielding box, is shown in Figure 3.11. 


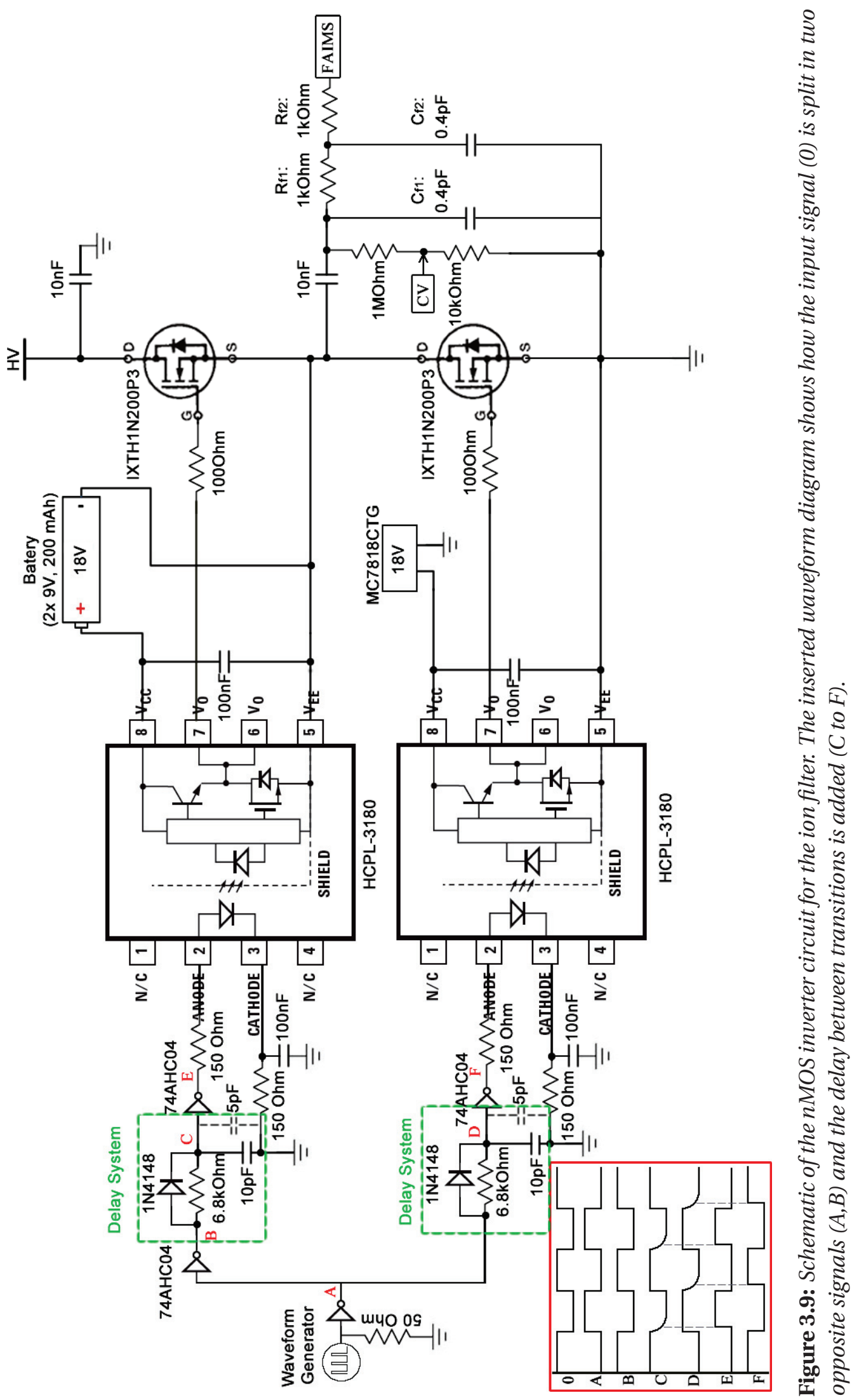




\subsubsection{Compensation Voltage electronics}

The compensation voltage (CV) ramp is computer generated using a data acquisition board (NIPCIe-6361). All ramp parameters, i.e. maximum and minimum voltage and time and voltage steps, are defined by the user using the LabVIEW ${ }^{\circledR}$ interface. However, the DAQ has a maximum analog output range of $\pm 10 \mathrm{~V}$. Therefore, a customized amplifier was fabricated to allow a larger scanning range. A schematic of the $\mathrm{CV}$ amplifier circuit are presented in Figure 3.10. The designed amplifier has a gain of $\mathrm{x} 2$ and a maximum output range of $\pm 20 \mathrm{~V}$. An additional OpAmp was placed at the output of the CV amplifier to add the lock-in modulation signal to the ramp. The second stage gain was set to $\mathrm{x} 1$ to avoid modifying the CV ramp. Both stages can be separated to work independently. Finally, a low pass filter with cutoff frequency of $1 \mathrm{kHz}$ was added to the circuit's output to reduce the noise levels.

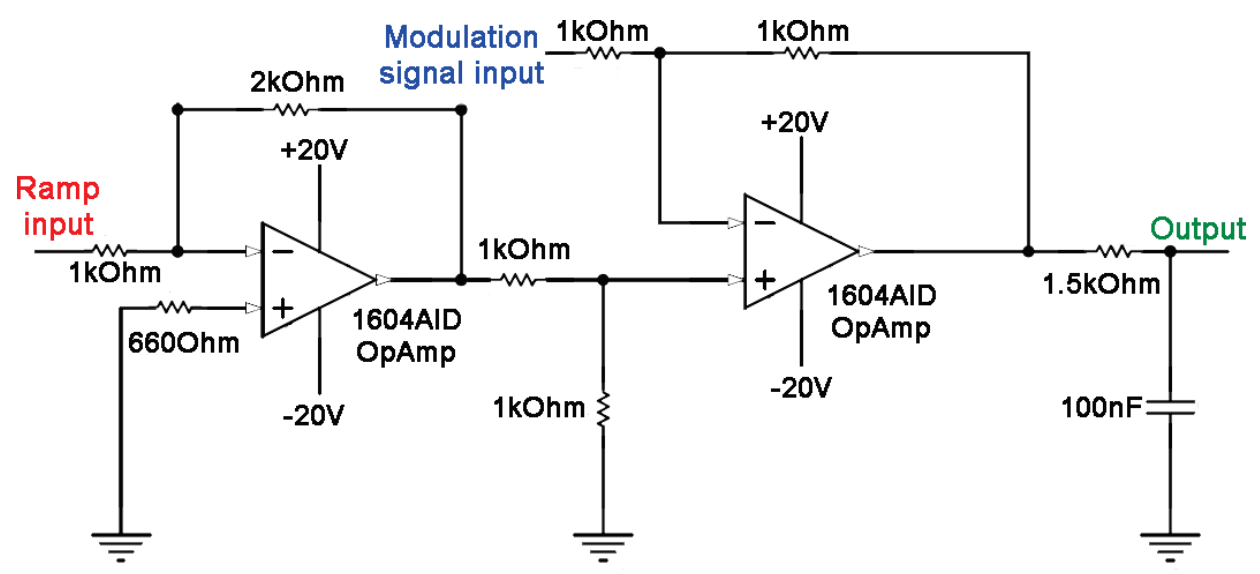

Figure 3.10: Schematic of the compensation voltage amplifier including the stage to add the modulation signal.

\subsubsection{Ion detector}

The ion detection region is composed of a pair of electrodes $(7 \times 8 \mathrm{~mm})$ surrounded by the ground electrodes. The bottom electrode is connected to a voltage source which generates the electric field for deflecting the ions towards the detection electrode. The detection electrode is connected to a low noise current amplifier with a gain of $10^{11}$. The amplified signal is then digitalized by a NI PCIe-6361 data acquisition board and recorded using a customized LabVIEW $^{\circledR}$ software. The interface software allows the user to monitor the detected signal, save the acquired data and input some of the measurement parameters, e.g. number of samples, sample rate and number of measurements. The software's block diagram is shown in appendix C.

Figure 3.12 shows the schematic of the designed low noise current amplifier circuit. It has two sequential stages: a current to voltage converter followed by a non-inverting amplifier. The feedback resistor (R1) of the I-to-V converter was set to $1 \mathrm{G} \Omega$ for a gain of $10^{9}$. This gain is added to the $10^{2}$ gain of the non-inverting amplifier for a total of $10^{11}$. The values of the implemented devices are summarized in Table 3.1. The cut off frequencies of the low pass 


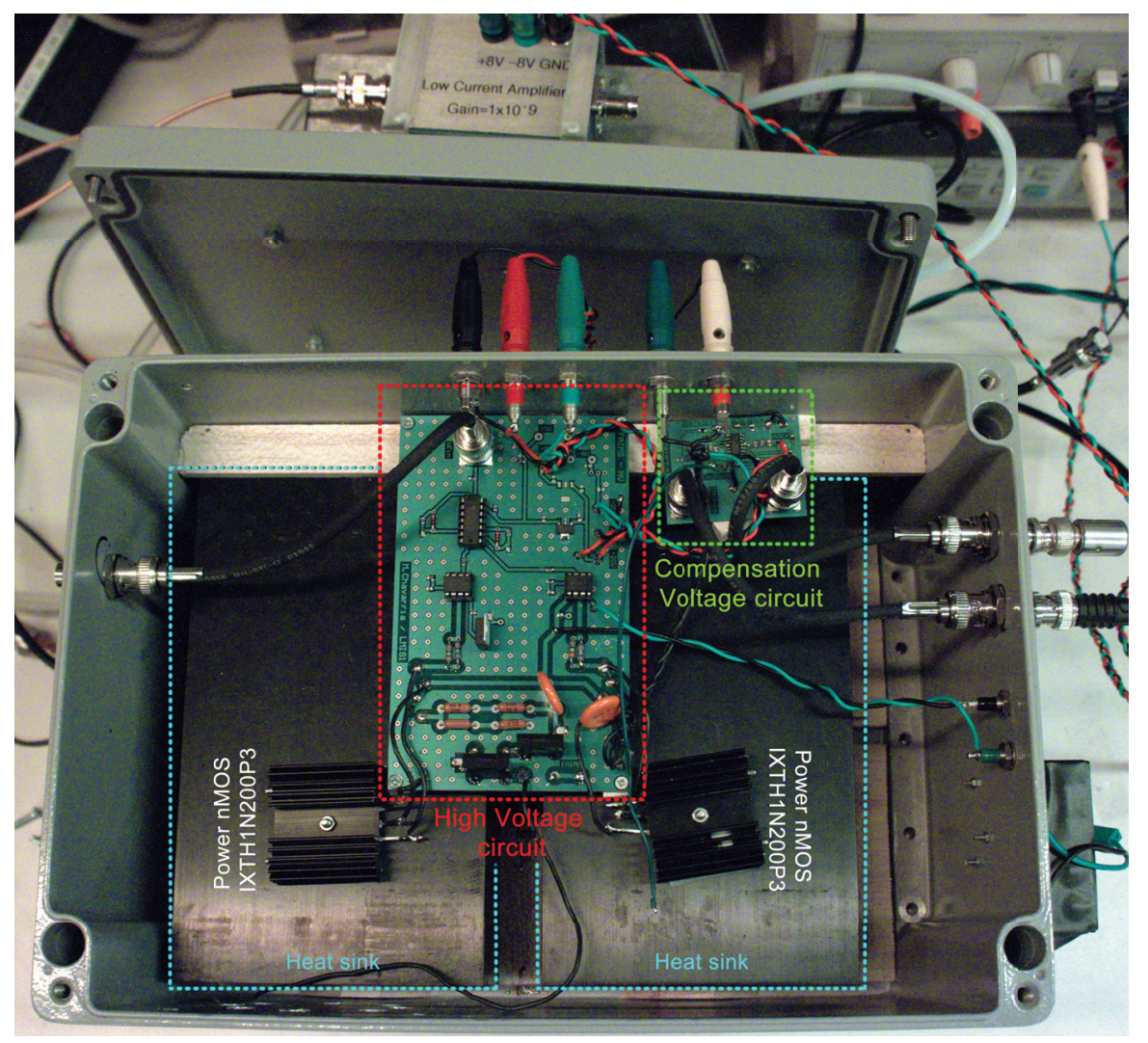

Figure 3.11: Picture of the fabricated high voltage and compensation voltage circuits for the ion filtering. Both circuits are shielded inside a metallic box for protection and to reduce the coupled noise into the detected signal.

filters ( $R 1 C 1, R 4 C 2$ and $R 5 C 3$ ) were fixed at $1 \mathrm{kHz}$. Even though it is recommended to have most of the gain in the first amplification stage to reduce the amplification of the noise, the gain of the I-to- $\mathrm{V}$ converter was kept to a maximum of $10^{9}$ to avoid parasitic capacitance problems, i.e. increasing the gain to $10^{10}$ will require a feedback resistor of $10 \mathrm{G} \Omega$, at this resistance levels the parasitic capacitances are around $0.1 \mathrm{pF}$, almost one order of magnitude higher than the required capacitance value $(0.015 \mathrm{pF})$ to keep the LP filter's $f_{c}$ at $1 \mathrm{kHz}$.

\begin{tabular}{|l|c|c|c|}
\hline$R_{1}[\Omega]$ & $C_{1}[\mathbf{p F}]$ & $R 3 / R 2$ & Total Gain $[\mathbf{V} / \mathbf{I}]$ \\
\hline $1 \times 10^{6}$ & 150 & 100 & $1 \times 10^{8}$ \\
\hline $1 \times 10^{7}$ & 15 & 100 & $1 \times 10^{9}$ \\
\hline $1 \times 10^{8}$ & 1.5 & 100 & $1 \times 10^{10}$ \\
\hline $1 \times 10^{10}$ & 0.2 & 100 & $1 \times 10^{11}$ \\
\hline
\end{tabular}

Table 3.1: Summary of the tested low noise current amplifier parameters

In order to ensure low electric noise for the system, the LMC6001 ultra-low input current operational amplifier was selected. This OpAmp has an input referred current noise $\left(i_{n}\right)$ of 


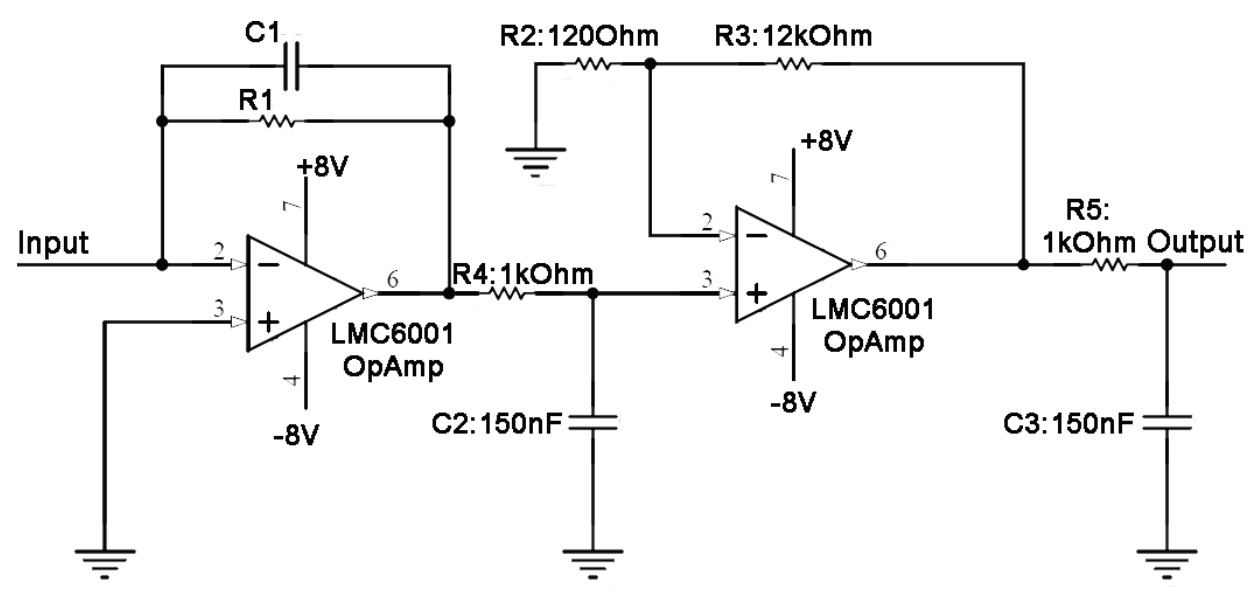

Figure 3.12: Schematic of the low noise current amplifier circuit.

$0.13 \mathrm{fA} / \mathrm{Hz}^{0.5}$ and an input referred voltage noise $\left(e_{n}\right)$ of $22 \mathrm{nV} / \mathrm{Hz}^{0.5}$. Therefore,the system's input referred noise is given by:

$$
\begin{aligned}
& I_{n}=\sqrt{\left(\frac{e_{n 1}^{2}}{R_{1}^{2}}+i_{n}^{2}+\left(T R_{n}\right)^{2}\right)+\left(\frac{e_{n 2}^{2}}{R_{1}^{2}}\right)} x \sqrt{B W} \\
& T R_{n}=\sqrt{\frac{4 k_{B} T}{R_{1}}}
\end{aligned}
$$

where $k_{B}$ is the Boltzmann's constant in Joules per Kelvin, $T$ the resistor's absolute temperature in Kelvin, $T R_{n}$ the thermal noise from the I-to-V converter's feedback resistor, $B W$ the systems band width in Hertz, $e_{n 1}$ and $e_{n 2}$ the input referred voltage noise from the first and second amplification stages.

For the designed amplifier, the theoretical model predicts that the thermal noise $\left(T R_{n}\right)$ will dominate the output noise at medium and high frequencies. Therefore, the input referred current and voltage noise can be neglected. However, at low frequencies, the OpAmp's input referred voltage noise increases and cannot be neglected anymore. This can be compensated by increasing the gain of the I-to- $\mathrm{V}$ converter. Figure 3.13 shows voltage noise versus frequency behavior of the selected OpAmp.

In order to confirm the validity of the proposed model, the calculated noise levels were compared to the experimentally measured noise spectral density of the fabricated low noise current amplifier. Several measurements were taken at gain levels from $10^{8}$ to $10^{11}$, i.e. $R_{1}=1 \times 10^{6}$ to $1 \times 10^{9} \Omega$. As shown in Figure 3.14, the noise spectral density measurements (straight lines) agree with the theoretical model (dashed lines): At low frequencies, the $1 / \mathrm{f}$ noise dominates the spectra. At medium frequencies, the noise stabilizes at a level fitting the calculated thermal noise. Finally, at high frequencies, the low pass filters attenuate the signal. Another observed phenomenon is the increase of the low frequency noise levels when the 
high voltage waveform of the ion filter is applied; however, at medium and high frequencies the spectra remain approximately constant.

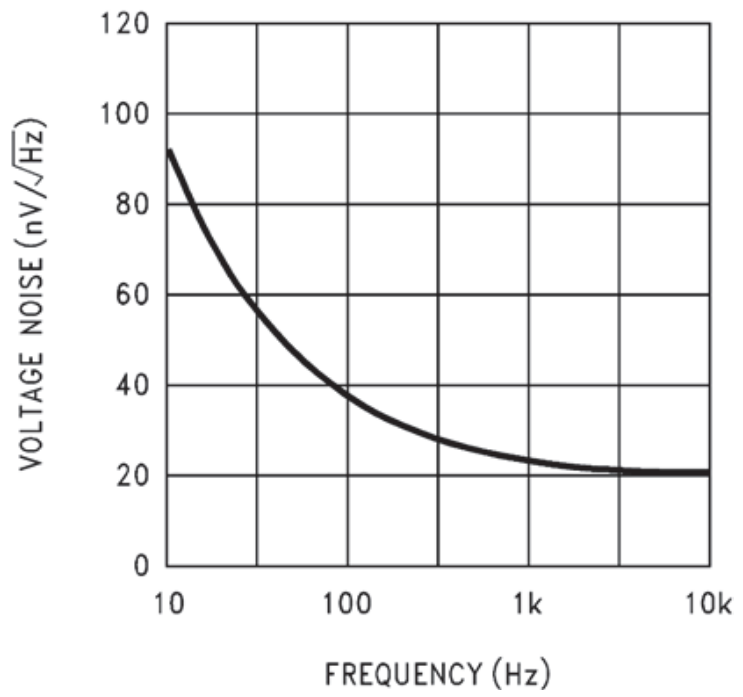

Figure 3.13: Voltage noise versus frequency behavior of the LMC6001 Ultra-Low Input Current Amplifier [8].

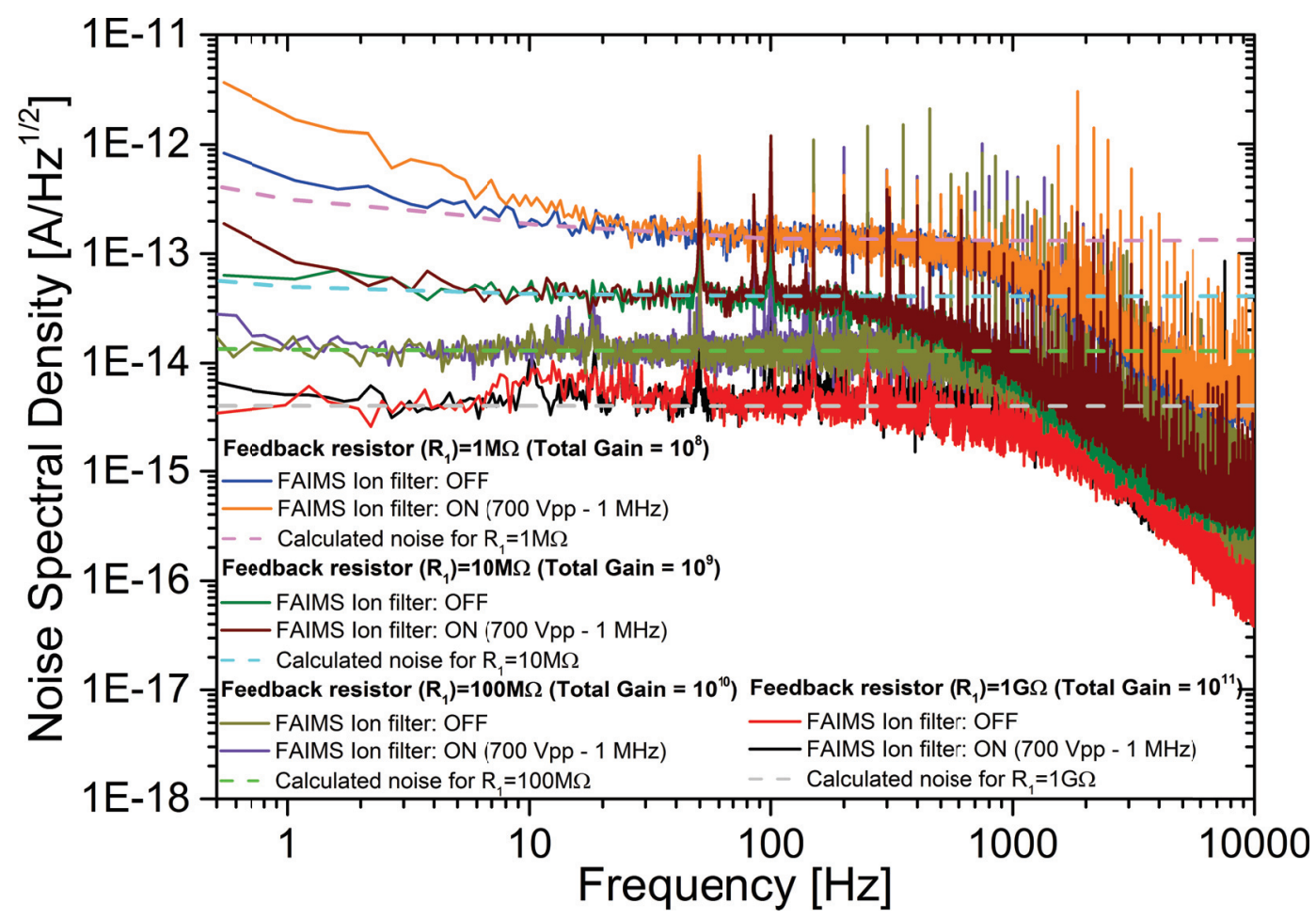

Figure 3.14: Comparison of the current noise spectral density of the fabricated amplifier with different gains. The dashed lines represent the calculated theoretical input referred noise using Equation 3.1. These measurements were performed with zero ion current. 


\subsubsection{Lock-in Amplifier}

As can be seen from the low noise current amplifier noise spectra in Figure 3.14, the noise levels decrease at medium and high frequencies. Therefore, being able to measure the ion current at higher frequencies with a narrow bandwidth will filter the 1 /f noise and considerably improve the signal to noise ratio (SNR). With this aim, the lock-in detection technique was implemented in the FAIMS measurements.

Lock-in amplifiers use a modulation signal to extract small signals from very noisy environments. By adding a reference signal with a specific frequency and phase to the input signal, it is possible to identify the component of interest from the background. Other component signals, i.e. noise, with different frequencies or phase are attenuated [48, 49].

A basic block diagram describing the working principle of a common lock-in amplifier is presented in Figure 3.15. First, the input signal is multiplied by a reference signal, usually supplied by an internal oscillator. The signal is then filtered by an analog low pass filter or digitally averaged over a specific time. In the second case, the system bandwidth is given by the inverse of the averaging time. The averaging time must be set much longer than the signal period to cancel all the unwanted components of the original signal.

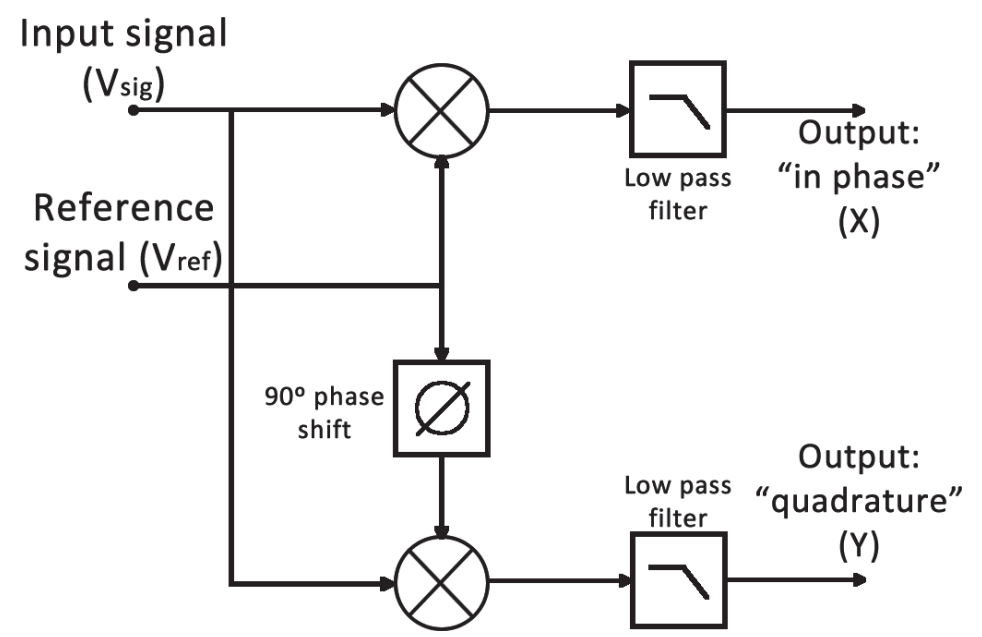

Figure 3.15: Basic blocks diagram of a lock-in amplifier.

More complex systems have an additional detector that works in a similar way to the first detector except that it shifts the reference signal's phase by 90 degrees. The two phase lock-in amplifiers have two outputs: the real or "in-phase" component $(\mathrm{X})$ and the imaginary or "quadrature" component (Y). The main advantage of this kind of amplification is that the phase adjustment does not have to be made a-priori. The measurements phase shift can be mathematically changed from the measured data. Also, the measurements' phase dependency can be removed by calculating the magnitude of the signal vector $[48,49]$.

Figure 3.16 shows the modified FAIMS setup for lock-in detection. A commercial DSP Lock-in amplifier (SR830) from Stanford Research Systems was selected for the synchronous modulation/demodulation of the FAIMS signals. The modulation signal could be applied to different sections of the FAIMS system, e.g. the Ionization source, the analyte flow, the deflection 


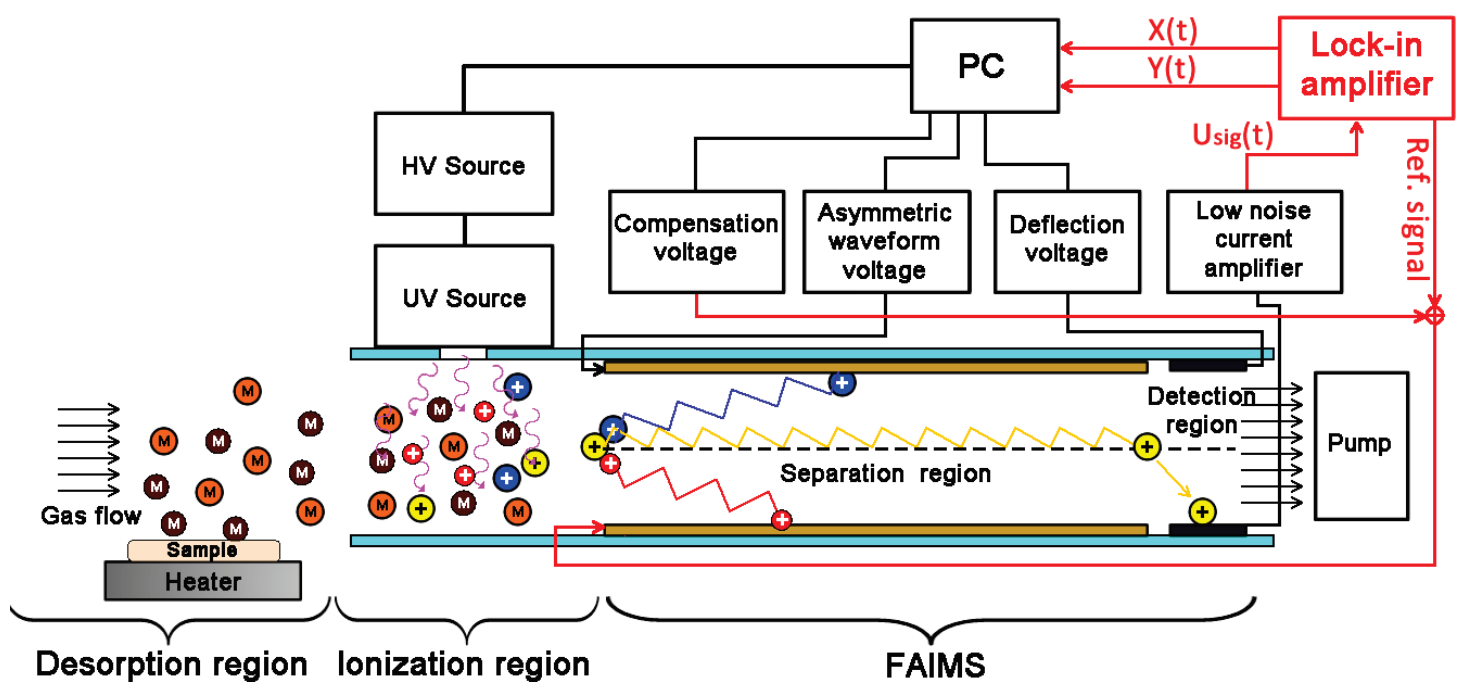

Figure 3.16: Schematic of the modified FAIMS setup with synchronous detection.

voltage, etc. However, performing the modulation in the filter region is the simplest approach which ensure the stability of the system. Therefore, the reference signal from the lock-in amplifier is added to the compensation voltage ramp using the differential amplifier in Figure 3.10 .

The detected ion currents are first amplified by the low noise current amplifier and then processed by the lock-in amplifier. The output signals are then digitalized by a DAQ and recorded by a PC. The designed LabVIEW ${ }^{\circledR}$ software was modified to simultaneously acquire both output signals ( $\mathrm{X}$ and $\mathrm{Y}$ ). Once the signal is acquired, it has to be processed to remove the phase shift. Finally, the signal is integrated to recover the original peak shape of the detected FAIMS spectrum. Figure 3.17 shows an example of the different data processing steps.
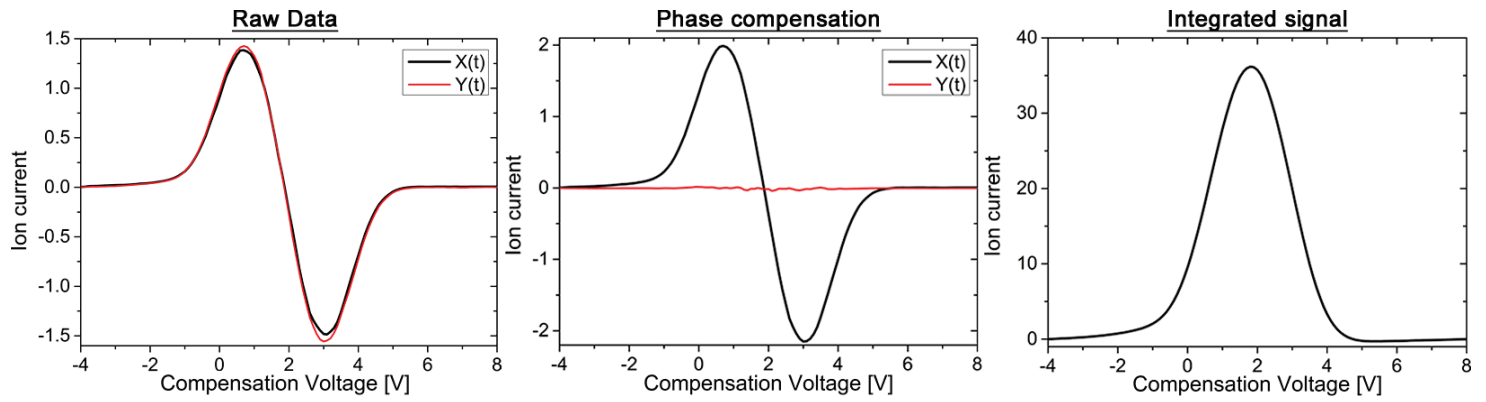

Figure 3.17: Different processing steps for the acquired lock-in signal.

\subsection{Conclusions}

A complete FAIMS system has been designed and fabricated. Also, a customized measurement setup was developed, including a commercial V-OVG calibration gas generator, pressure, humidity and flow sensors and a gas pump. This allowed precise control of different measurement conditions, such as analyte concentration, carrier gas pressure and flow rate. 
Four FAIMS cores were fabricated and tested, using different geometries, materials and ionization sources: The characterization of the systems confirmed that the implemented fabrication methods and materials did not affect the FAIMS behavior, i.e. both alumina and PCB cores showed similar results. Also, the plates geometries with opposite face connectors had lower coupled noise levels than the designs with side connectors. The implemented corona and UV ionization sources had similar ionization efficiencies. However, in terms of stability and repeatability, the UV source exhibited a better performance.

Several of the peripheral systems required for the FAIMS measurements were also developed: The designed ion filter electronics allow the generation of rectangular voltage waveforms up to $1000 \mathrm{~V}_{\mathrm{PP}}$ in the $\mathrm{MHz}$ range and compensation voltage ramps from -20 to $+20 \mathrm{~V}$. An ultra-low noise- low current amplifier with a total gain of $\times 10^{11}$ was fabricated to enhance the FAIMS sensitivity. Also, a lock in amplifier was added to the FAIMS setup to modulate the detected signal and, therefore, improve the systems' SNR. Finally, each subsystem was shielded in a metallic box to protect them and the user and to reduce the coupled noise from internal and external sources. The complete FAIMS system is presented in Figure 3.18.

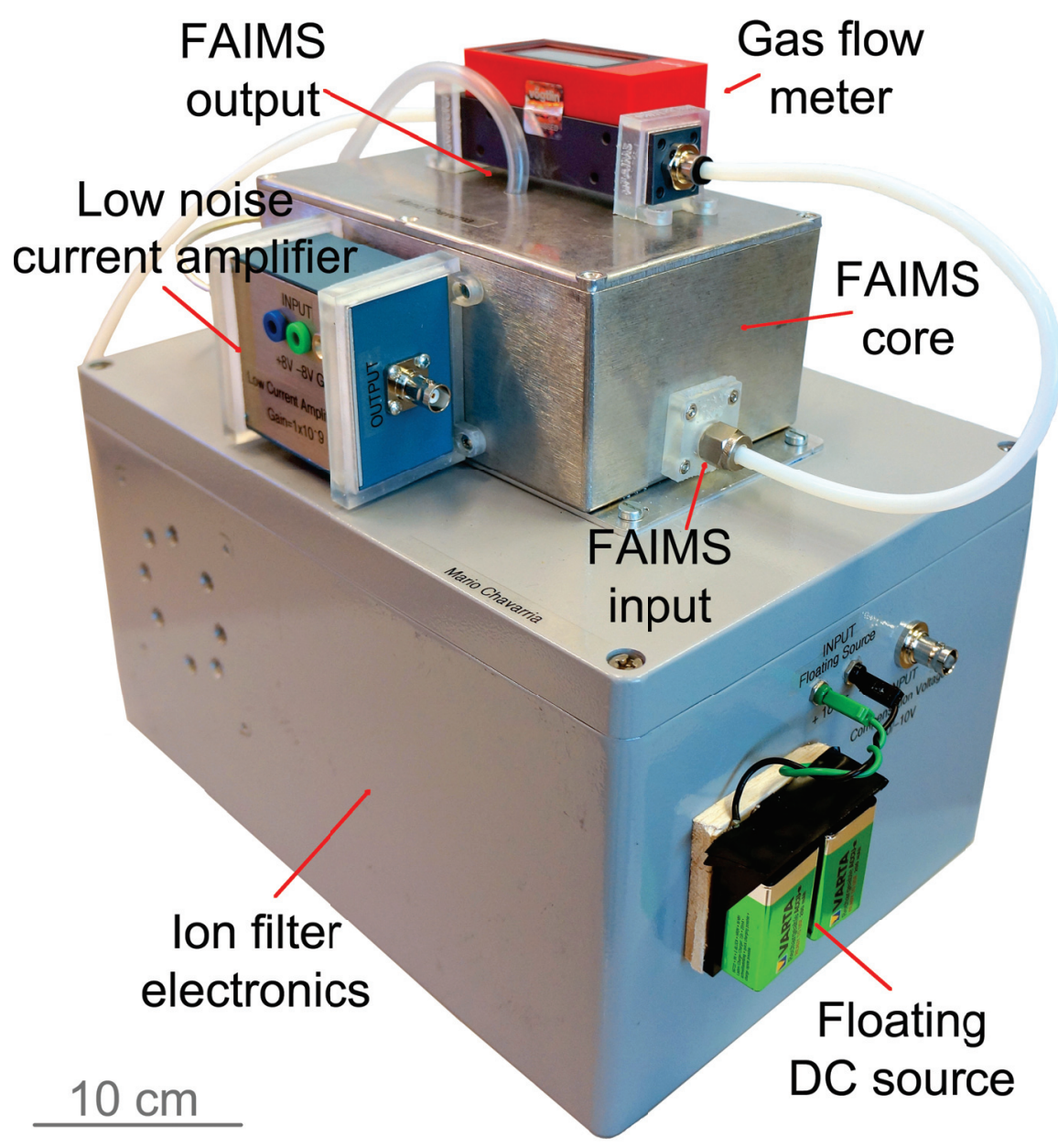

Figure 3.18: Fabricated FAIMS system with the shielding covers. 



\section{Experimental results}

In this chapter, the effects of different experimental and media parameters on the FAIMS sensitivity and resolution are presented. Each section focuses on one of the main FAIMS subsections: ionization, ion filter and ion detection regions. Finally, the limit of detection of the developed systems is determined. All the experiments included in this chapter, unless stated otherwise, were performed at room conditions using a planar FAIMS with an UV ion source, a $250 \mu \mathrm{m}$ thick spacer and alumina substrates with $12 \times 10 \mathrm{~mm}^{2}$ filter electrodes.

\subsection{Ionization rate}

A key task in the development of a highly sensitive FAIMS is to keep the ratio between detected ions and injected molecules as high as possible. However, many factors can affect this rate. Therefore, in order to identify the best ionization/detection conditions, an experimental study on the ionization efficiency and ion losses was performed. In this section, a summary of the most significant results of the ionization study are presented.

\subsubsection{Effects of the UV source input current on the ionization efficiency}

The ionization efficiency directly depends on the incoming light flux. Consequently, increasing the number of photons produced by the UV lamp will improve the sensitivity of the FAIMS system. The best way to increase the photons emission, without modifying or replacing the UV lamp, is to increase the lamp's input current. However, high current levels might reduce the lamp's working lifetime or, if the maximum current level is exceeded, damage it.

The ratio between the input current and the produced light flux is not always linear or equal for all the UV lamps. Therefore, an initial measurement of the output light flux was taken at four input current levels. The generated light power was measured by placing an UV photodiode (SG01S-C18) at the output of the UV lamp. The diode's current was amplified using a commercial low noise current amplifier (EG \& G 5182). The red curve in Figure 4.1 shows the normalized variation of the lamp's output power at different input currents. As expected, the lamp's output increases with the input current level, reaching up to three times the value with the nominal current, but the power increase is not proportional to the input current. 


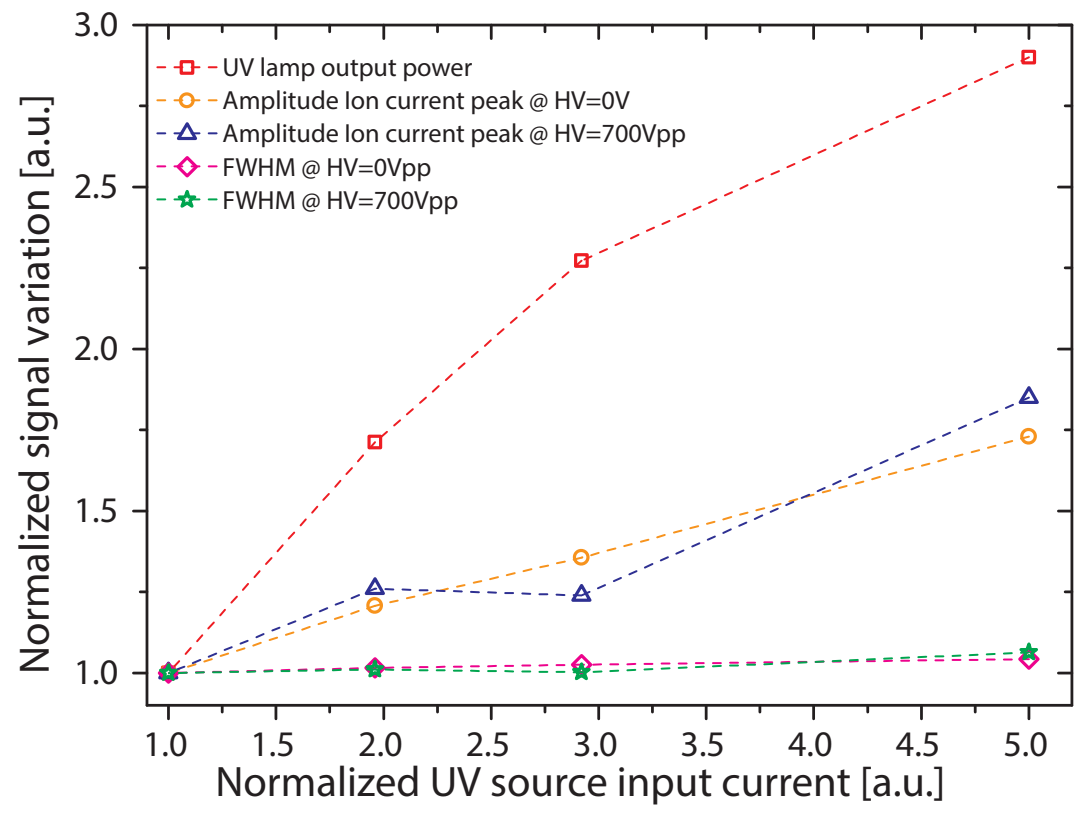

Figure 4.1: Variation in the UV lamp's output power and the detected ion current amplitude and FWHM at different input currents. The dashed lines between the points are only visual guides, not fitted lines or theoretical modeling of the physical behavior.

In order to evaluate the effect of the higher photons flux in the FAIMS sensitivity, several acetone measurements were performed at different UV lamp's current levels. Two sets of measurements were taken, with (Figure 4.2.b) and without (Figure 4.2.a) ion filtering, to recognize any possible influence of the high voltage signal on the spectra's variation. In both cases, the detected ion current increased with the lamp's input current. However, the ion current increase is less than half the increase of the measured light power. The orange and blue curves in Figure 4.1 show the normalized variation in the amplitude of the detected acetone spectra. The cause of the variation difference between the measured output power and the ion current in not clear. Among the possible explanations are: a non linear behavior of the ionization photon emission at the ionization frequencies or an uneven variation rate of the different components of the emitted light spectra.

The FAIMS resolution is given by the ratio between the CV shift and the peak's FWHM. Since the ion peak shift in $\mathrm{CV}$ was not affected by the increase in the ionization rate, any variation in the FAIMS resolution will only depend on the peak's FWHM. The FWHM should remain as low as possible to avoid reducing the system's resolution. The magenta and green curves in Figure 4.1 show the normalized variation in the FWHM of the detected acetone spectra. The maximum increase in the FWHM during these measurements was about $5 \%$. This variation is small compared with the gain in signal amplitude and will not seriously affect the FAIMS resolution.

In summary, increasing the UV source input current improves the FAIMS sensitivity without affecting its resolution. However, an increase of a factor of five in the input current leads to an improvement of less than a factor of two in sensitivity. 

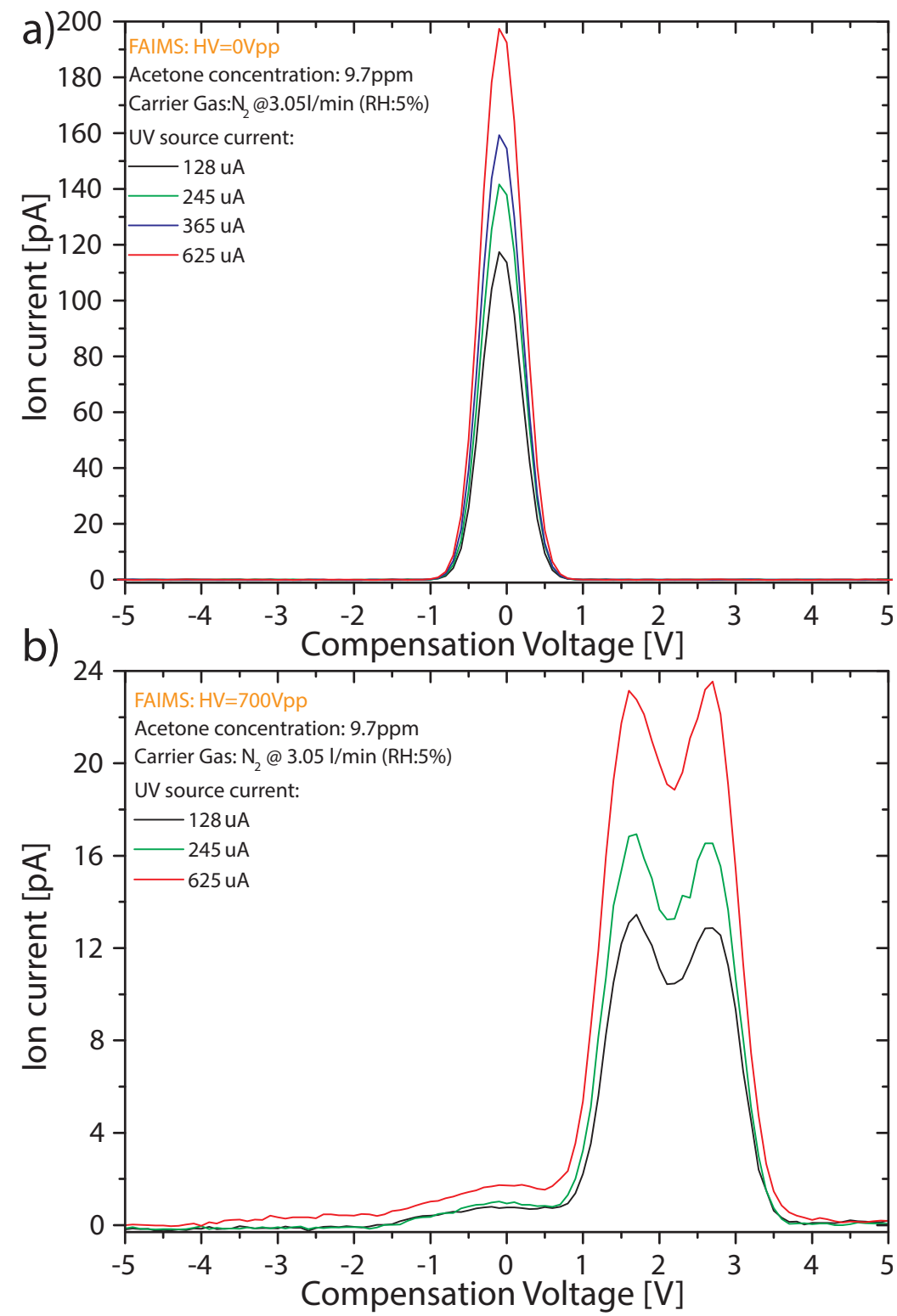

Figure 4.2: Detected acetone spectra using different UV source input currents. The spectra in (a) were not filtered by the FAIMS high voltage signal. The spectra in (b) were filtered by a $700 V_{P P}$ high voltage signal at $1 \mathrm{MHz}$ and 33\% duty cycle. Each curve is the average of 10 measurements under the same conditions.

\subsubsection{Effects of Carrier gas composition on the ionization efficiency}

Another option to improve the ionization efficiency is to reduce the amount of light absorbed by the media. The media's absorption cross section can vary by several orders of magnitude depending on the incoming light's wavelength and the composition of carrier gas. Therefore, in order to maximize the ionization efficiency, it is essential to choose a carrier gas with an absorption cross section as low as possible for the ionization wavelength. Table 4.1 presents some absorption cross-section $(\sigma)$ examples of gases commonly used in FAIMS for the imple- 
mented UV source's wavelength, i.e. $123.6 \mathrm{~nm}$. Nitrogen, with an absorption cross-section of about $7 \times 10^{-22}$, is one of the best candidates to use as a carrier gas [3]. However, it is important to avoid any contamination in the carrier gas that might increase the average absorption cross-section of the media. Figure 4.3 shows an example of the ionization efficiency reduction when the amount of contaminants, i.e. water vapor, in the carrier gas increases. As expected, the amplitude of the detected ion peak decreases when the relative humidity $(\mathrm{RH})$ of the carrier gas increases. The ion filter was turned OFF to avoid influence of the high voltage signal on the spectra's variation. From Equation 2.3, the theoretical light absorption from the top to the bottom of the ionization region is about $2 \%$ and $20 \%$ for nitrogen with $\mathrm{RH}$ levels of $5 \%$ and $40 \%$, respectively. This agrees with the diference in amplitude of the measured ion peaks at the mentioned RH levels, i.e. $30 \%$. The higher amplitude reduction might be caused by an additional amount of light absorbed by the acetone molecules or other contaminants present in the carrier gas.

\begin{tabular}{|l|r|}
\hline Molecule & $\sigma\left[\mathbf{c m}^{2} /\right.$ molecule $]$ \\
\hline Acetone $\left(\mathrm{C}_{3} \mathrm{H}_{6} \mathrm{O}\right)$ & $3.90 \times 10^{-17}$ \\
\hline Oxygen $\left(\mathrm{O}_{2}\right)$ & $4.16 \times 10^{-19}$ \\
\hline Nitrogen $\left(\mathrm{N}_{2}\right)$ & $7.00 \times 10^{-22}$ \\
\hline Hydrogen $\left(\mathrm{H}_{2}\right)$ & $7.00 \times 10^{-20}$ \\
\hline Water vapor $\left(\mathrm{H}_{2} \mathrm{O}\right)$ & $6.20 \times 10^{-18}$ \\
\hline
\end{tabular}

Table 4.1: Absorption cross-sections of different gases used in FAIMS for the wavelength of the implemented UV source (123.6 $\mathrm{nm})$ [3]

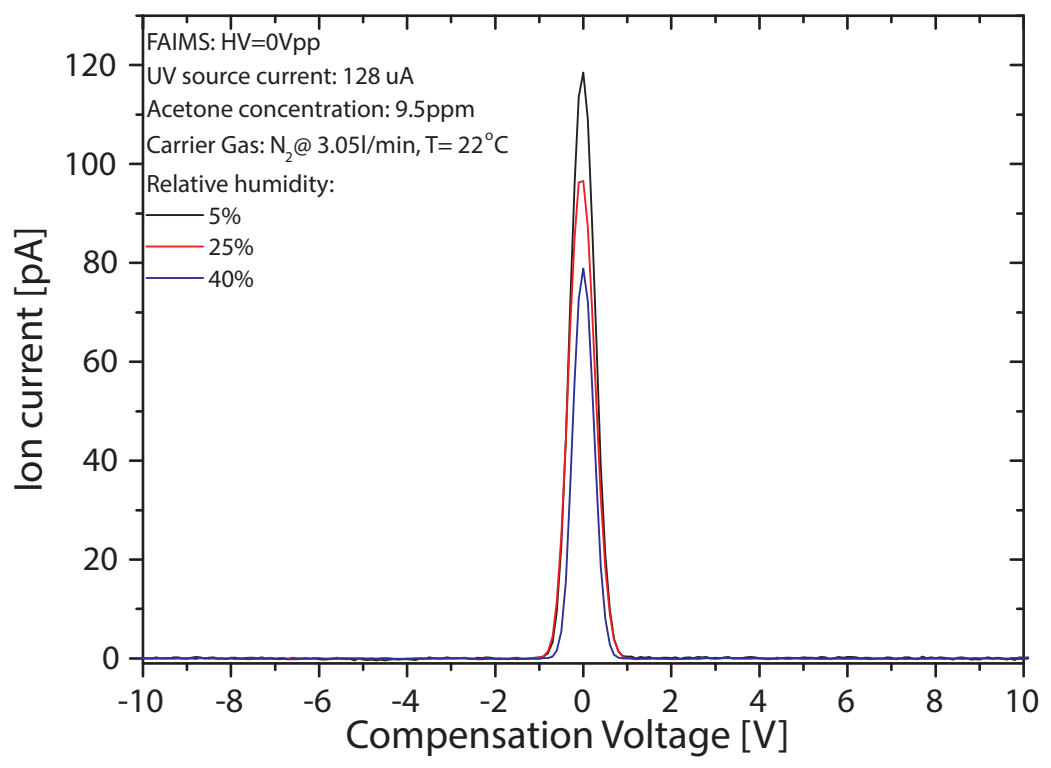

Figure 4.3: Detected acetone spectra using nitrogen as a carrier gas with different levels of humidity. The measurements were taken without the ion filter signal. Each curve is the average of 10 measurements under the same conditions. 


\subsubsection{Effects of the carrier gas flow rate on the ionization efficiency}

The probability of a molecule being ionized depends on many factors, including the time it spends in the ionization region. Molecules crossing faster the ionization region will have a lower ionization probability than the slower ones, leading to lower ionization efficiencies. However, fast molecules also have lower probability of recombination and diffusion into the electrodes. This reduces the total amount of ion losses in the path towards the detector.
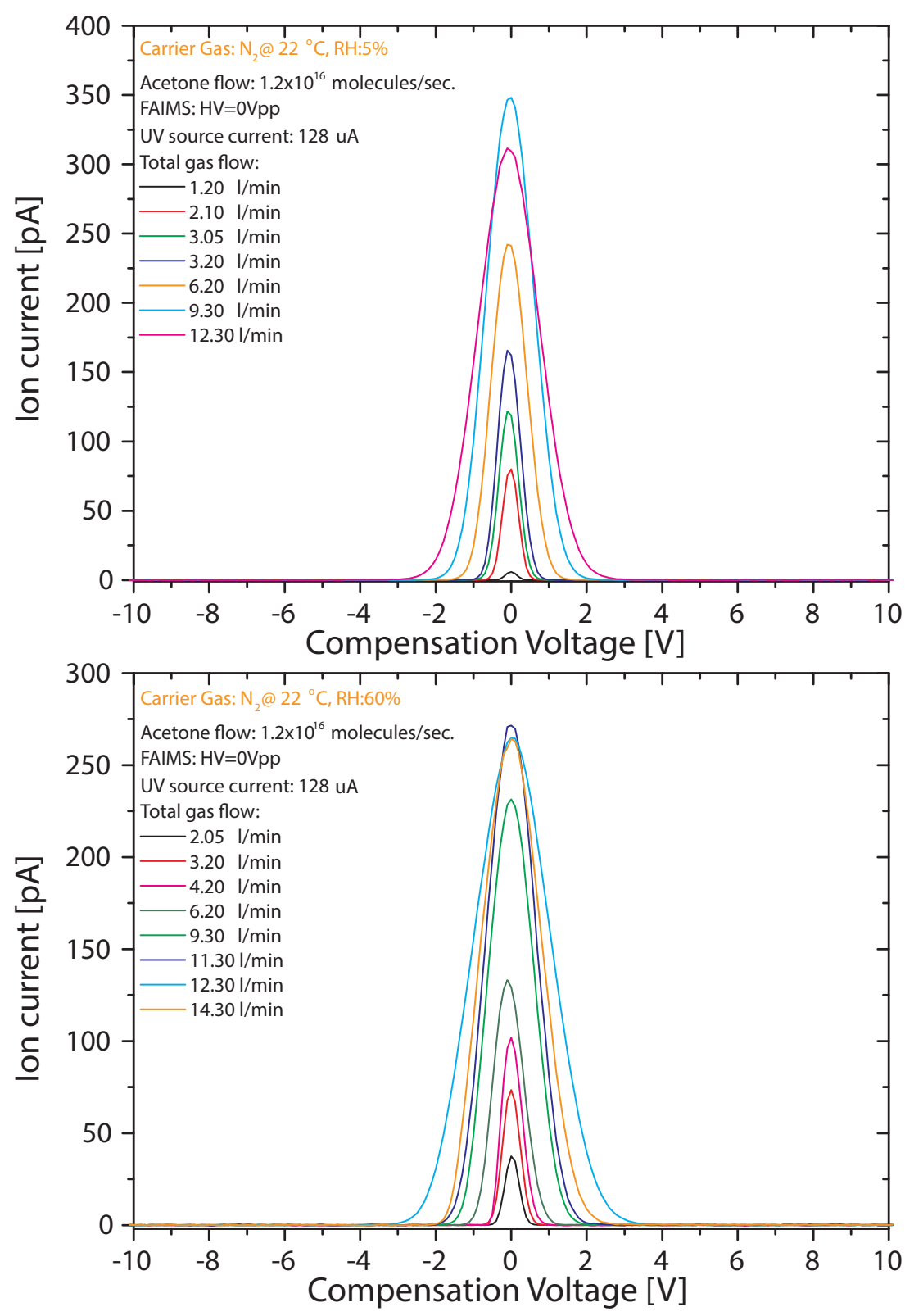

Figure 4.4: Detected acetone spectra at different carrier gas flow rates. The analyte flow is constant for all measurements: $1.2 \times 10^{16}$ acetone molecules per second. The measurements were taken at high (b) and low (a) humidity levels without the ion filter signal. Each curve is the average of 10 measurements under the same conditions. 
A set of experiments was performed to identify the optimal analyte input velocity. For these measurements, the analyte flow was set to $1.2 \times 10^{16}$ acetone molecules per second and the carrier gas flow was varied from 1 to 14 liters per minute, i.e. from $\sim 4.2 \times 10^{20}$ to $\sim 5.9 \times 10^{21}$ molecules/s. This way, the flow velocity variation is compensated by an opposite change in the analyte molecules' density, maintaining the analyte flow constant. Figure 4.4 shows the detected ion peaks at different nitrogen flow rates at high (b) and low (a) humidity levels. The ion filter was turned off to avoid external interference. The normalized variation of the ions peak amplitude and FWHM is summarized in Figure 4.5. At flow velocities lower than 10 $1 / \mathrm{min}$, the flow velocity variation has a greater affect in the ion loss rate than in the ionization efficiency. In other words, increasing the carrier gas flow rate leads to a reduction in the ion losses higher than the reduction in the ionization efficiency. Therefore, the amount of ions reaching the detector increases. Nevertheless, at about $101 /$ min the reduction in the ionization rate exceeds the ion losses decrease and the detected ion current starts decreasing. In sum, by correctly tuning the carrier gas flowrate, the ratio between the amount of detected ions and the input flow of analyte molecules can be improved by more than one order of magnitude, i.e. this ratio increased from $\sim 10^{-9}$ to $\sim 10^{-7}$ at gas flowrates from 1.2 to $9.31 / \mathrm{min}$, respectively. Increasing the flow velocity also reduces the time the ions spend between the filter electrodes. This reduces the filtering effect, allowing more ions to escape the separation region. Therefore, the ion peaks become wider when the gas flowrate increases, as shown in Figures 4.4 and 4.5. This is a non-desired effect, since the increase in the FWHM reduces the FAIMS resolution. Consequently, a tradeoff between the sensitivity improvement and resolution decrease must be determined according to the measurement requirements.
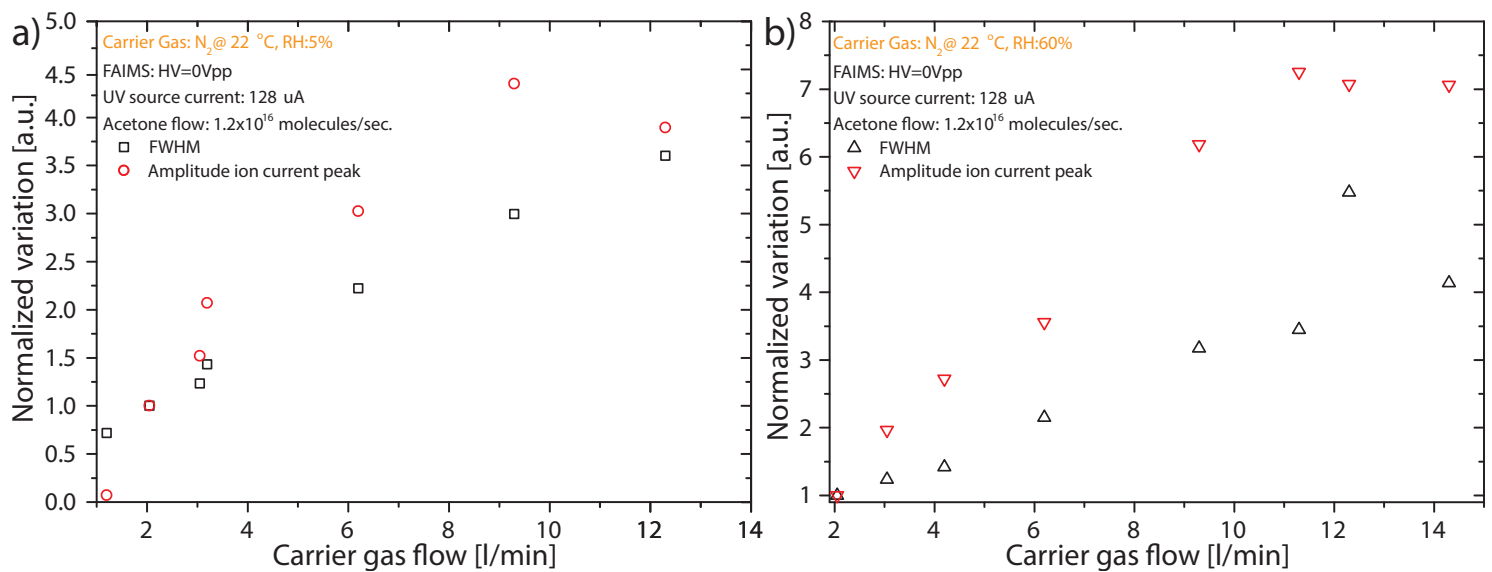

Figure 4.5: Variation of the detected ion peak's amplitude and FWHM at different carrier gas flow rates and a constant analyte flow. Each point is the average of 10 measurements under the same conditions.

\subsubsection{Ion losses}

The ion losses in the FAIMS channel highly reduce the ratio between detected and produced ions. The main causes of ion losses in a neutral media are recombination and diffusion. In order to determine the amount of ion losses between the ionization region and the detector, 
the ion current was monitored in different points along the channel. The measurements ware preformed using the fabricated electrode plates shown in Figure 3.5.c. Additionally, an schematic of the measurements setup is presented in Figure 4.6. One electrode was connected to the input of the low current amplifier while its counter electrode was connected to the defection voltage source.

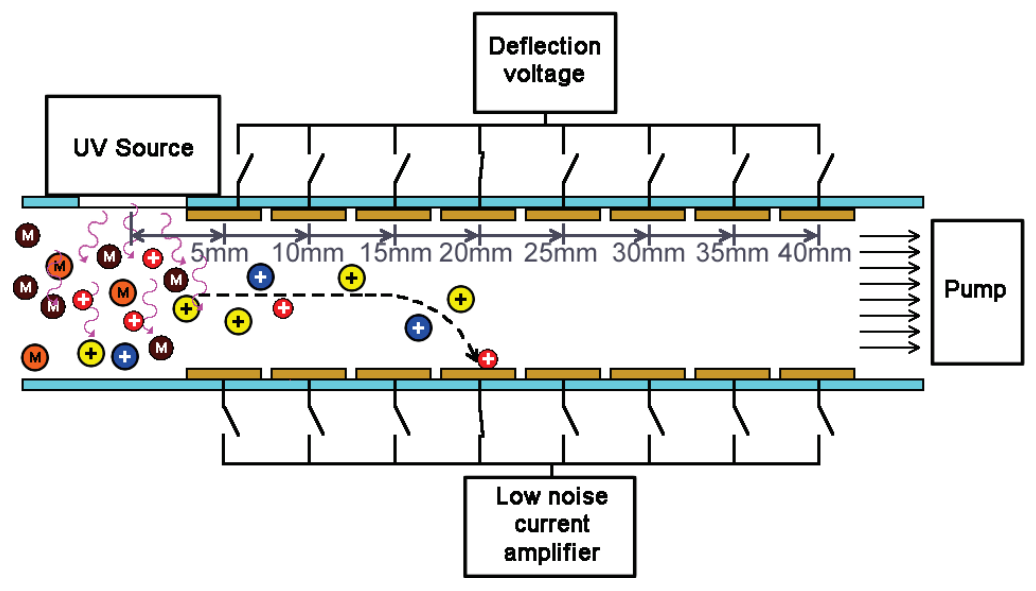

Figure 4.6: Schematic of the ion losses experimental setup.

When an ion collides with one of the electrodes, it gets neutralized. Then, depending on its absorption/desorption characteristics, the neutralized analyte molecule can get attached to the surface or continue traveling with the carrier gas. Therefore, the measurements were performed sequentially, activating only one electrode pair at the time. This way, the interference from the other electrodes can be reduced. The average current levels at each position are presented in Figure 4.7. The highest ion loss rate was observed for the measurements at $9 \mathrm{ppm}$ acetone concentration with a carrier gas flow of $3 \mathrm{l} / \mathrm{min}$ at room pressure. The ion current decreased up to two orders of magnitude along the channel, i.e. from 5 to $40 \mathrm{~mm}$ measured from the center of the ionization to the center of the detector electrode. Conversely, the lowest ion loss rate was observed on the low pressure and high gas flowrate measurements, i.e. $75 \%$ along the channel.

Decreasing the ion density reduces the probability of interaction between the ions. Consequently, the ions loss rate will also decrease, as can be seen in Figure 4.7. The ion density can be decreased by different methods: reducing the ionization efficiency, e.g. lowering the ion UV source input current (red and black curves), decreasing analyte input (magenta curve) or reducing the pressure inside the channel (green curve). Despite the reduction in the loss rate, all these approaches will decrease the total amount of ions reaching the detector. The reasons behind these phenomena are explained in sections 4.1.1, 4.2.5 and 4.4.

Another option to minimize the ion losses without reducing the detected ion current is to reduce the travel time of the ions inside the channel. This can be achieved by decreasing the distance between the ionization region and the detector or by increasing the carrier gas flow rate (blue curve). These approaches can highly improve the FAIMS sensitivity. However, reducing the time the ions spend in the filter region also reduces the system's resolution, as 
explained in sections 4.1.3, 4.2.6 and 4.2.7.

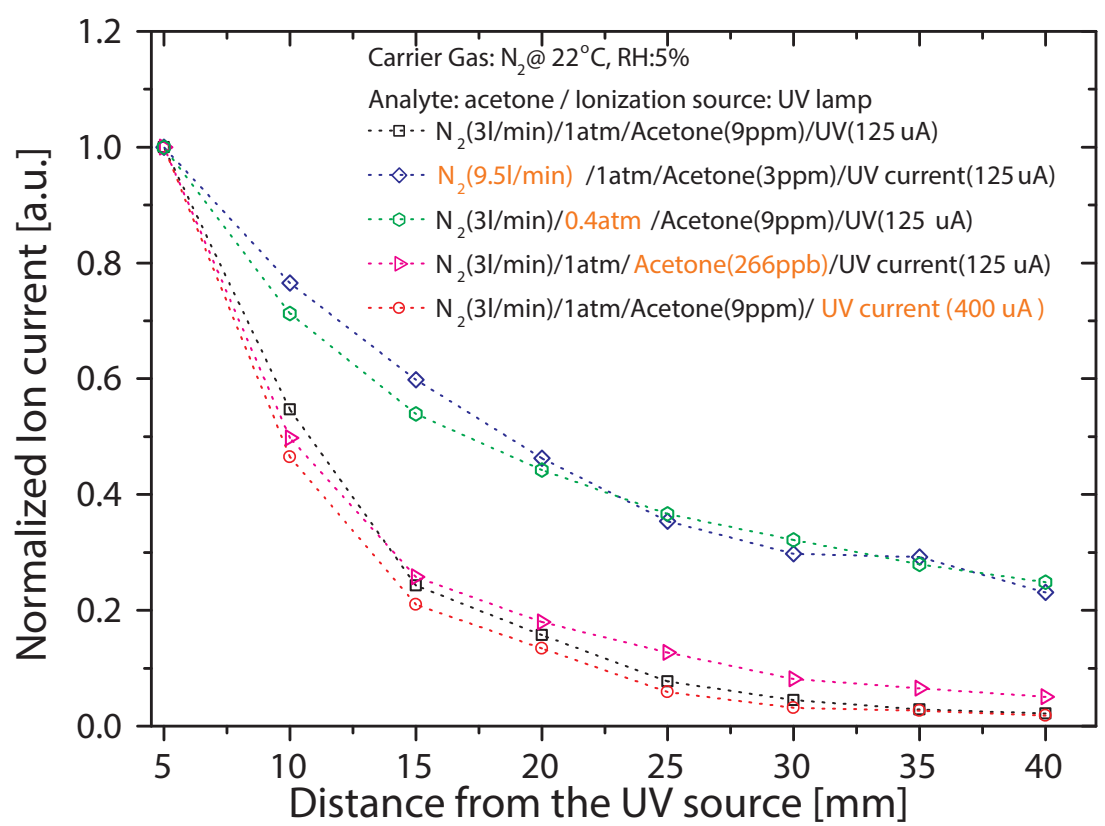

Figure 4.7: Reduction in the ion current inside the FAIMS channel due to the ions recombination and diffusion. Each curve was taken at different conditions, modifying only one parameter at the time. The distance in the X axis is measured from the center of the ionization region to the center of the detection region. The dashed lines between the points are only visual guides, not fitted lines or theoretical modeling of the physical behavior. Each point is the average of 10 measurements under the same conditions.

\subsection{Ion filter}

The ion filtering in FAIMS is performed at high electric fields and high frequencies. This greatly disturbs the ions' paths, thereby reducing the ions' flow towards the detector. It also couples high amounts of electronic noise into the ion detector electronics. Therefore, the ion filter system must be meticulously tuned to minimize its influence in the system's sensitivity. The following section presents the tuning and experimental characterization of the different ion filter parameters and their effects on the FAIMS sensitivity.

\subsubsection{Noise reduction}

A high level of electronic noise can hide the detected ion signals. Thus, in terms of sensitivity in FAIMS, reducing the coupled noise is as important as increasing the molecules detection efficiency. With this aim, an ultra-low noise amplifier was designed (Section 3.3.4). The ion filter HV signal greatly increases the noise levels in the detected spectra. Therefore, an analogue low pass (LP) filter was added to the output of the ion filter circuit, shown in Figure 3.9. All values of the tested LP filter's components and the resulting $f_{c}$ s are summarized in Table 4.2.

The LP filter's order and cutoff frequency should be carefully selected to minimize the noise 
a)

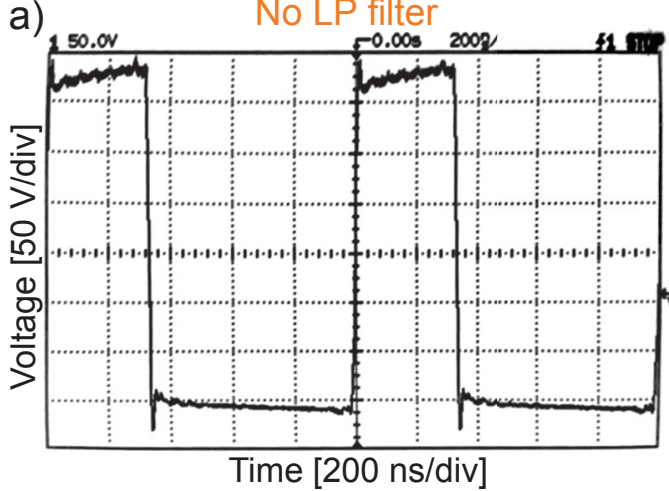

c)

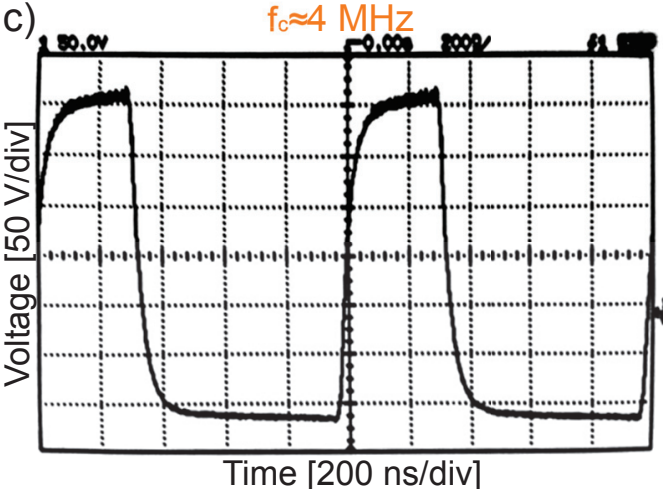

b)

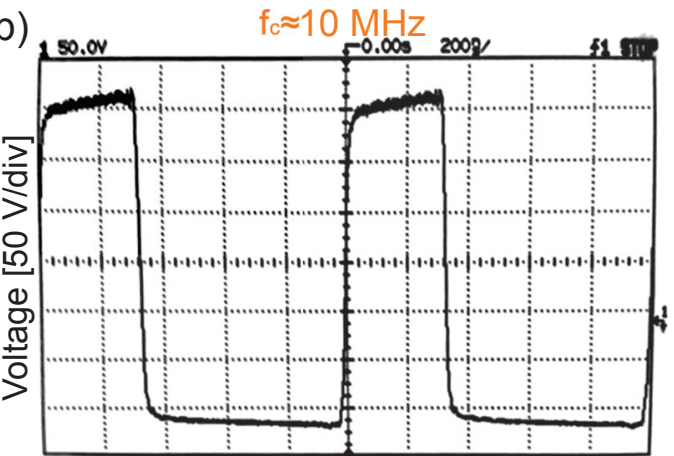

Time $[200 \mathrm{~ns} / \mathrm{div}]$

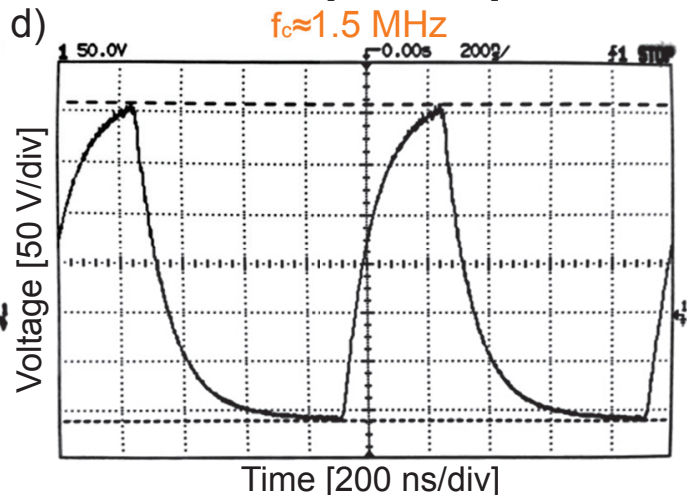

Figure 4.8: Influence of the low pass filter's cutoff frequencies in the HV ion filter waveform. Lower $f_{c}$ decrease the signal noise but also deforms the waveform. The signals in all measurements were set to $300 V_{P P}$ at $1 \mathrm{MHz}$ and $33 \%$ duty cycle. The cutoff frequencies $\left(f_{c}\right)$ were calculated from the measured HV waveforms.

levels without considerably affecting the FAIMS resolution: designing a filter with a low cutoff frequency $\left(f_{c}\right)$ will decrease the amount of coupled noise. However, it will also slow down the circuit's response, deforming the output voltage waveform (Figure 4.8).

\begin{tabular}{|l|c|c|}
\hline$R_{f 1}=R_{f 2}$ & $C_{f 1}=C_{f 2}$ & $f_{c}$ \\
\hline $200 \Omega$ & $0.4 \mathrm{pF}$ & $10 \mathrm{MHz}$ \\
\hline $1000 \Omega$ & $0.4 \mathrm{pF}$ & $4 \mathrm{MHz}$ \\
\hline $3000 \Omega$ & $0.4 \mathrm{pF}$ & $1.5 \mathrm{MHz}$ \\
\hline
\end{tabular}

Table 4.2: Summary of the tested LP filter components. The cutoff frequencies $\left(f_{c}\right)$ were calculated from the measured HV waveforms (The circuit schematics are included in Figure 3.9).

Different LP filters of first and second order and cutoff frequencies between 1.5 and $10 \mathrm{MHz}$ were tested. The experiments were performed using acetone as analyte and air as the carrier gas. The ion filter parameters were set to $700 \mathrm{~V}_{\mathrm{PP}}$ at $1 \mathrm{MHz}$ and $33 \%$ duty cycle. All the detected spectra are presented in Figure 4.9.a. These experiments showed a decrease on the noise levels of more than one order of magnitude when the LP filter is implemented. However, at lower $f_{c}$, 
the deformation of the high voltage waveform reduces the ion filter capabilities. This leads to a decrease of the CV shift and an increase of the ions peak's amplitude.
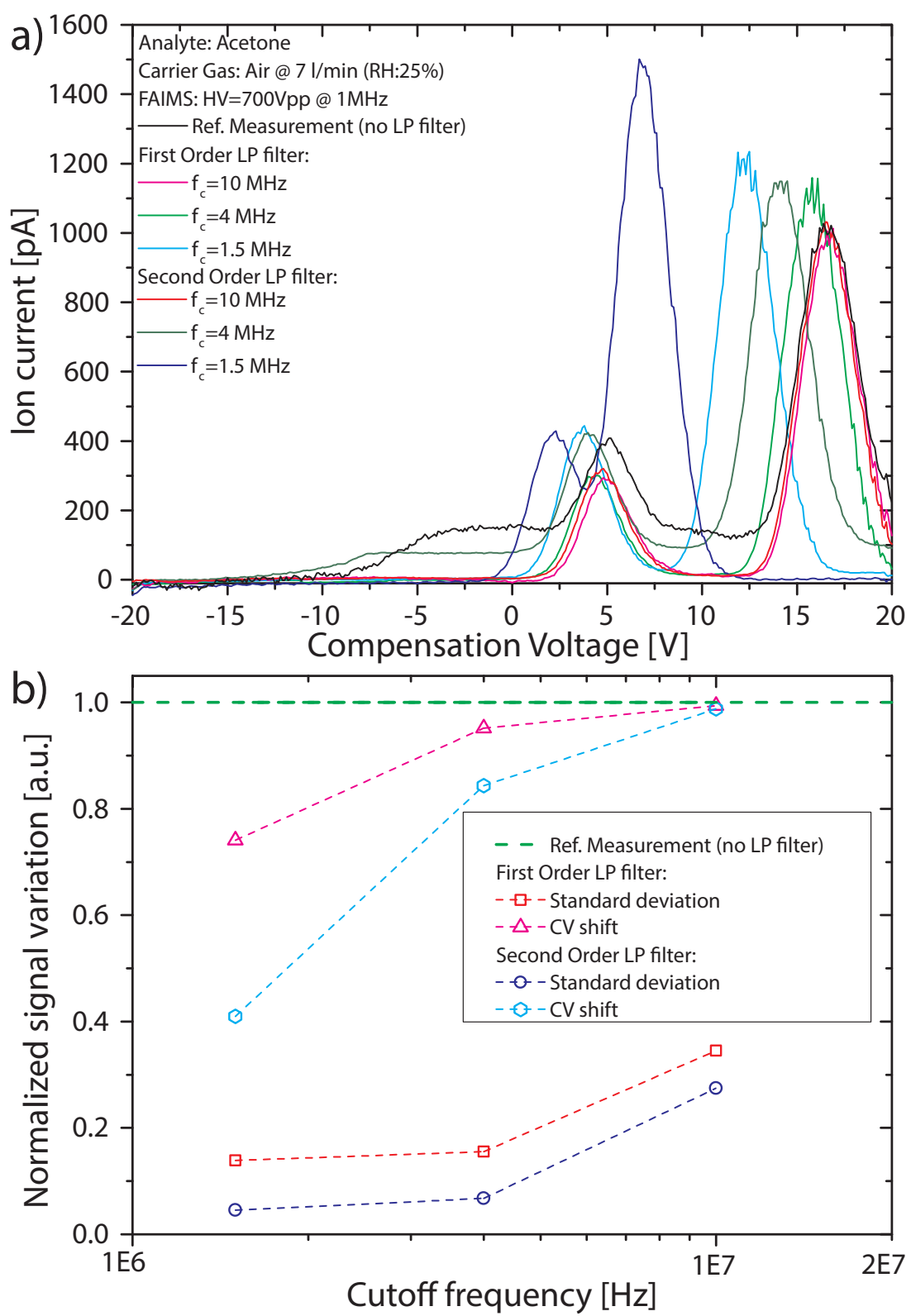

Figure 4.9: Effects of the LP filter's cutoff frequency on the detected acetone FAIMS spectra. The detected spectra are shown in (a) and the variation in the ions peak's amplitude and CV shift are summarized in (b). All measurements were perform with a FAIMS ion filtering of $700 V_{P P}$ at $1 \mathrm{MHz}$ and $33 \%$ duty cycle. Air was used as carrier gas at room conditions, i.e. $1 \mathrm{~atm} ., 22^{\circ} \mathrm{C}$ and $25 \%$ relative humidity. The dashed lines between the points are only visual guides, not fitted lines or theoretical modeling of the physical behavior.

Figure 4.9.b summarizes all the normalized CV shift and noise level variations at different $f_{c}$. As can be seen, the noise level saturates at frequencies of $4 \mathrm{MHz}$ or lower. Also, the CV shift 
starts to decrease rapidly at $f_{c}$ higher than $4 \mathrm{MHz}$. Consequently, implementing a second order LP filter with an $f_{c}$ of $4 \mathrm{MHz}$ is the best tradeoff between the increase in sensitivity and the resolution loss, i.e. an SNR gain of around 13 times with a reduction on the CV shift of only $15 \%$.

Finally, in order to further decrease the coupled noise levels, the ion filter circuits, the low current amplifier and the FAIMS core were placed in separated shielded boxes. A comparison between the system's current noise spectral density, before (black) and after (red) implementing the LP filter and the shielding, is shown in Figure 4.10. The noise spectral density, at the same amplifier's gain $\left(1 \times 10^{8}\right)$, is more than two orders of magnitude lower than in previous measurements without shielding and filtering. This improves the expected FAIMS sensitivity levels by the same amount.

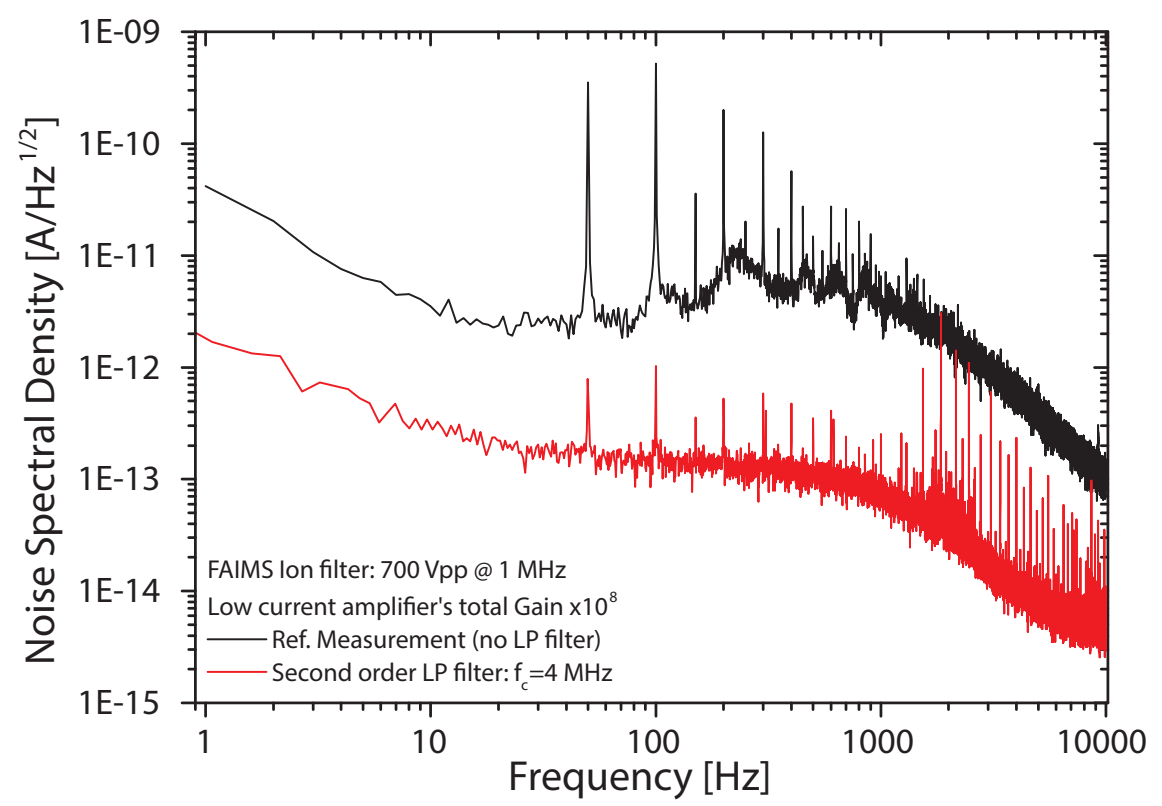

Figure 4.10: Comparison between the NSD before (black) and after (red) implementing the LP filter and the shielding boxes.

\subsubsection{Ion filtering at different separation voltages}

Increasing the magnitude of the ion filter separation voltage (HV), also known as the filtering voltage, enlarges the ion mobility variation between the low and high electric field regimes. This lead to larger CV shifts, improving the FAIMS resolution. Nevertheless, higher mobilities also increase the amplitude of the ions' oscillation inside the ions' channel, which reduces the system's sensitivity. This sensitivity decrease is represented as a reduction of the effective ions' gap, described by equation 2.11 .

Two examples of the separation voltage's influence on the detected FAIMS spectra are presented in Figures 4.11 and 4.12. The detected analytes are acetone (Figure 4.11) and o-xylene (Figure 4.12) at concentrations of 9.5 and $2.7 \mathrm{ppm}$ respectively. The separation voltage was increased from 0 to $750 \mathrm{~V}_{\mathrm{PP}}$ at $1 \mathrm{MHz}$ and 33\% duty cycle. All the measurements were performed with a nitrogen flow rate of $3.061 / \mathrm{min}$ and a $75 \%$ relative humidity. Each plot is the 
average of 5 scans with a sampling frequency of $12.3 \mathrm{kHz}$, sampling rate of 10 points/volt and averaging time of 0.1 seconds/ point. The increase in the separation voltage led to a total reduction of approximately 25 times in the ion peak's amplitude of both analytes during the entire measuring range. Conversely, the $\mathrm{CV}$ shift increased from 0 to $14.6 \mathrm{~V}$ for acetone and from 0 to $14.2 \mathrm{~V}$ for o-xylene.
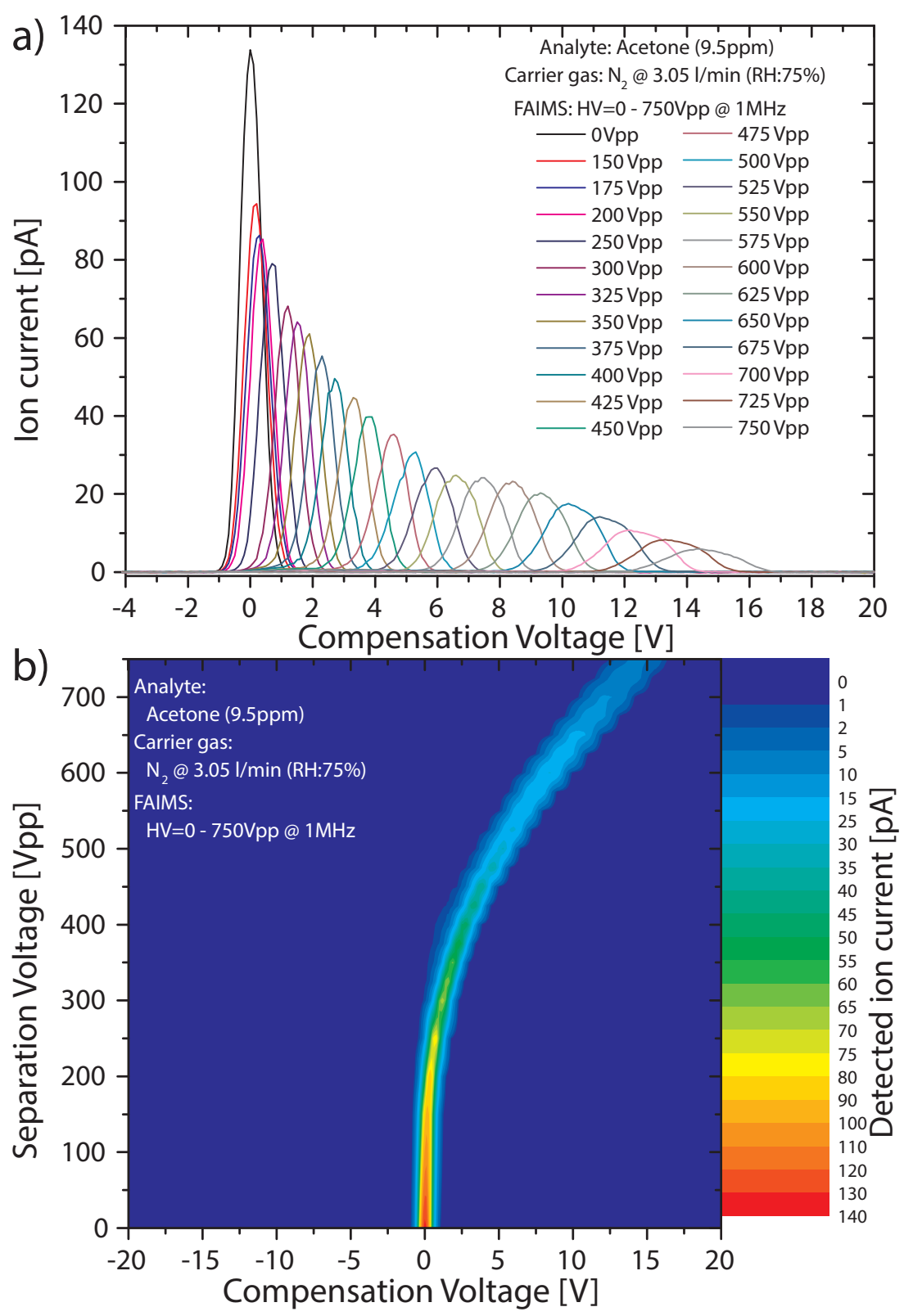

Figure 4.11: Effects of the separation voltage (HV) on the detected acetone spectra with a concentration of $9.5 \mathrm{ppm}$. The filter HV levels were increased from 0 to $750 V_{P P}$ at $1 \mathrm{MHz}$ and $33 \%$ duty cycle. In plot (a), the detected spectra at different HV levels are displayed as different curves. In plot (b), the detected spectra are placed along the " $Y$ " axis and the ion current is represented with a color scale. 

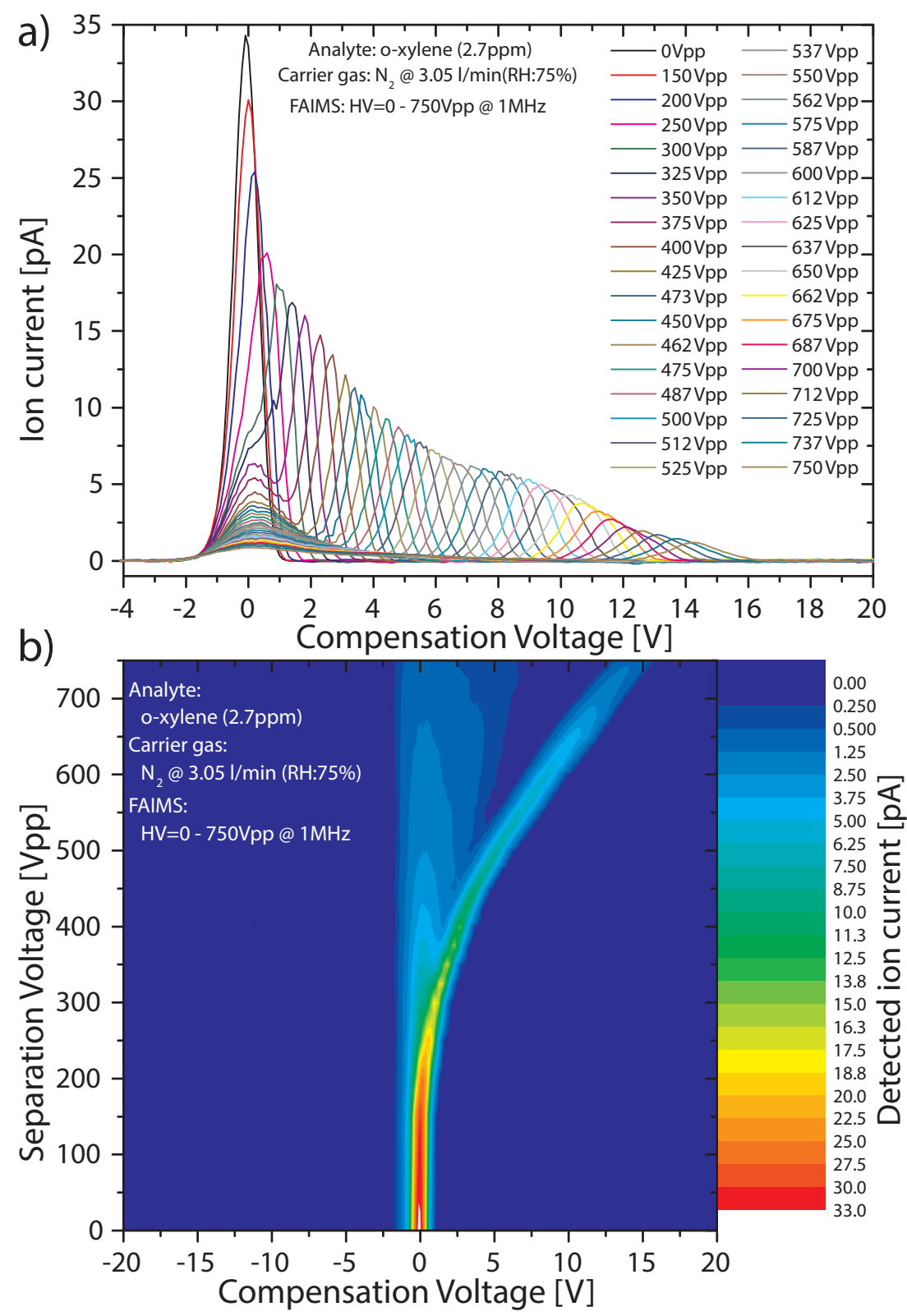

Figure 4.12: Effects of the separation voltage (HV) on the detected o-xylene spectra with a concentration of $2.7 \mathrm{ppm}$. The filter HV levels were increased from 0 to $750 V_{P P}$ at $1 \mathrm{MHz}$ and $33 \%$ duty cycle. In plot (a), the detected spectra at different HV levels are displayed as different curves. In plot (b), the detected spectra are placed along the " $Y$ " axis and the ion current is represented with a color scale.

The registered data is displayed using two types of 2D plots: the standard FAIMS graphs (Figures 4.11.a and 4.12.a) displays one measurement curve for each of the tested HV levels and the ion current values and CV values are depicted in the " $\mathrm{Y}$ " and " $\mathrm{X}$ " axes, respectively. This kind of plots allows one to clearly identifying all the detected ion peaks. However, using a contour graph, seen in Figures 4.11.b and 4.12.b, allows a more clear representation of the 
variation in the $\mathrm{CV}$ shift and, therefore, the ion mobility behavior. In these plots, the detected spectra are placed along the " $\mathrm{Y}$ " axis, the CV values are depicted in the "X" axis and the ion current is represented with a color scale.

\subsubsection{Humidity effects in the ion separation}
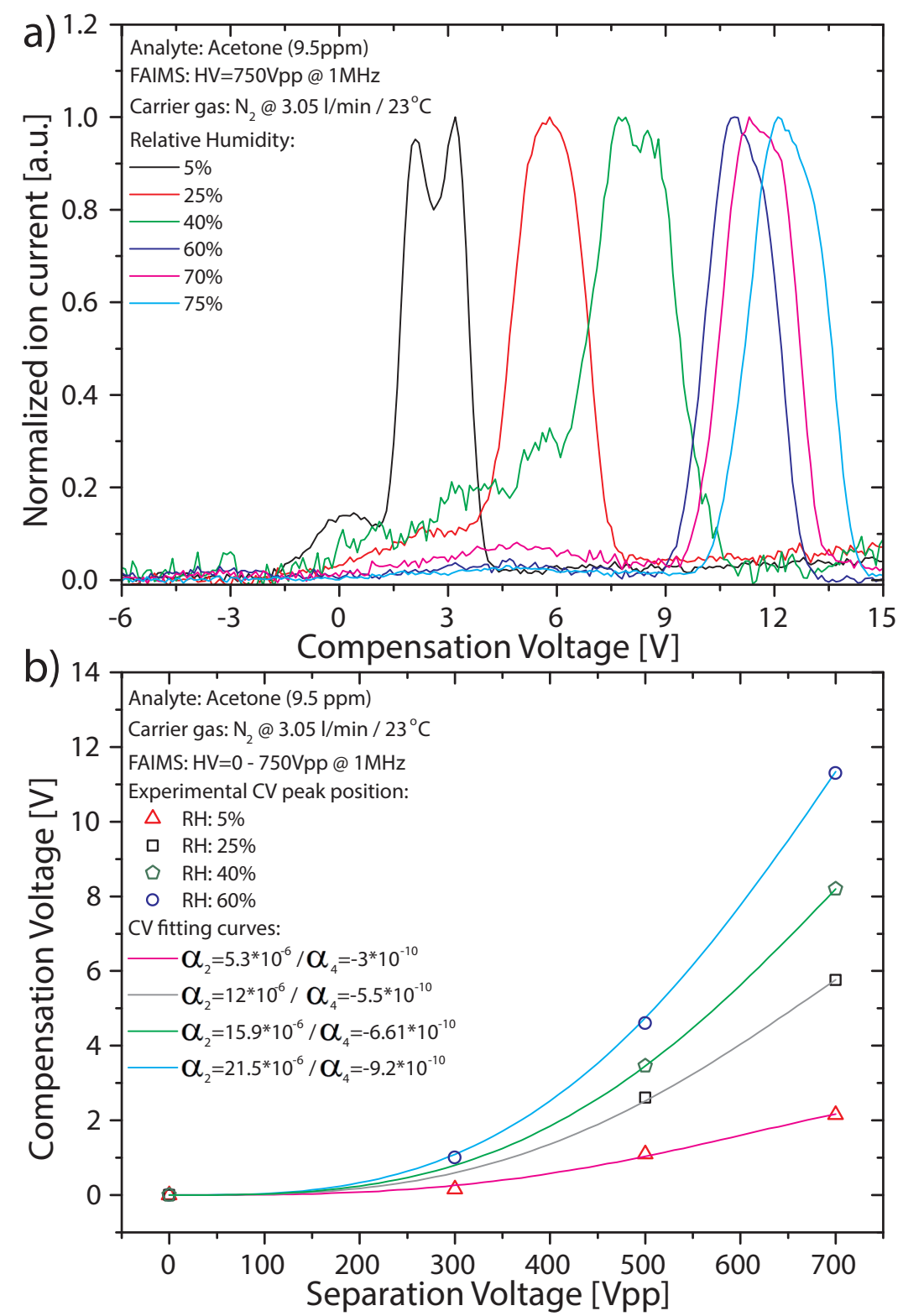

Figure 4.13: (a) Effects of the humidity levels in the detected acetone spectra. (b) Compensation Voltage shift-vs- separation voltage plots for acetone at different $R H$ levels.

The humidity level has important effects on the FAIMS spectra: A higher amount of water molecules in the carrier gas will increase the light absorption, thereby reducing the system's sensitivity, as explained in Section 4.1.2. It also increases the CV shift of the ion peaks, which 
improves the FAIMS resolution. Figure 4.13.a, shows an example of the acetone spectra detected under different humidity levels. Additionally, four "CV shift-vs- separation voltage" curves at RH levels from 5 to $75 \%$ are plotted in Figure 4.13.b. The experimental data was fitted using the alpha parameters' model in Section 2.3.2. The observed behavior is in good agreement with the results from other groups reported in literature $[25,7,30]$.

A tentative explanation for the variation in the ion peak's position is proposed in $[7,30]$. The authors claim that higher humidity levels increase the amount of interactions between the ions and the water neutrals leading to the formation of hydrated ions. The higher the humidity levels the higher the number of water molecules involved in the ion cluster. This changes the collision cross section between the ions and the carrier gas molecules and, consequently, the ion mobility dependency on the electric field.

\subsubsection{Influence of the filtering frequency on the ion separation}

One of the best approaches to improve the sensibility in FAIMS, without affecting the resolution, is to increase the ion filtering frequency. Higher frequencies reduce the amplitude of the ions' oscillation inside the channel, decreasing the ion losses. This is described by the effective ion gap model in section 2.3.1.

Figure 4.14 shows the detected acetone spectra (a) at different filtering frequencies and a summary (b) of the variation in the ions peak's amplitude and CV shift. All measurements were taken with a separation voltage of $700 \mathrm{~V}_{\mathrm{PP}}$ at 0.5 to $1 \mathrm{MHz}$ and $33 \%$ duty cycle. The nitrogen flow was set to $3.05 \mathrm{l} / \mathrm{min}$ at a temperature of $23{ }^{\circ} \mathrm{C}$ and relative humidity of $5 \%$. The ion filtering frequency was increased from $500 \mathrm{kHz}$ to $1 \mathrm{MHz}$ in steps of $100 \mathrm{kHz}$. Increasing further the frequency would require a faster switching device. Unfortunately, no commercially available transistors were found that were able to work at frequencies higher than $1 \mathrm{MHz}$ at the required voltage and current levels. The experimental results showed that by doubling the HV frequency (from $500 \mathrm{kHz}$ to $1 \mathrm{MHz}$ ), the FAIMS sensitivity increases more than 20 times. On top of this, the CV shift remained almost constant. The small decrease in the CV shift $(<8 \%)$ was caused by the deformation of the HV waveform at higher frequencies. This can be solved by implementing faster HV switching devices.

\subsubsection{Effects of the carrier gas pressure on the ion separation}

The ion mobility is inversely proportional to the media's number density $(\mathrm{N})$, as described in equation 2.5. Since the number density is directly proportional to the gas pressure, the ion mobility will increase at low pressures. Higher ion mobilities improve the FAIMS resolution but also decrease its sensitivity. Figure 4.15 shows the effect of the carrier gas pressure on the detected acetone spectra. The measurements were taken with pressures from 1 to 0.33 atm at a constant flowrate. The implemented experimental setup is shown in Figure 3.2: The pressure was decreased using a KNF gas pump (N816.3K) and the carrier gas and analyte flow rates were regulated by the mass-flow controllers in the Owlstone calibration gas generator. At $0.33 \mathrm{~atm}$, the CV shift increases five times in comparison to the measurements performed at room pressure. However, the signal amplitude decreases by about the same amount and a small broadening $(20-30 \%)$ of the ion peaks occurred at low pressures. This diminishes 
slightly the resolution improvement produced by the CV shift increase. Therefore, reducing the carrier gas pressure by three times led to an improvement of the FAIMS resolution of about a factor four.
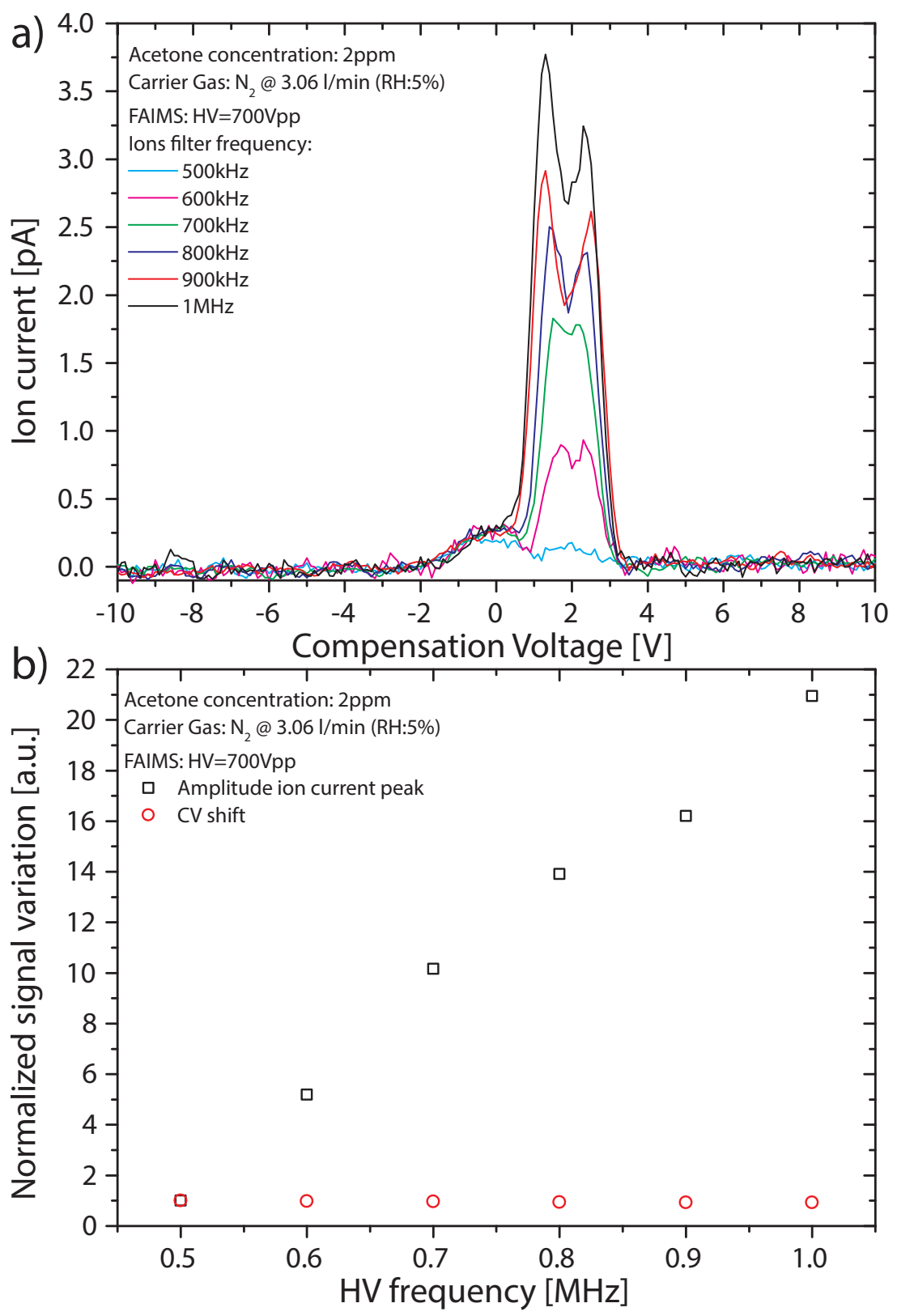

Figure 4.14: Effects of the HV waveforms' frequency on the FAIMS signal. (a) Detected spectra of 2 ppm of acetone at different filtering frequencies (1 scan per plot). The separation voltage was set to $700 V_{P P}$ with a $33 \%$ duty cycle. Nitrogen was used as carrier gas with a flowrate of 3.06 $l /$ min and relative humidity of 5\%. (b) Normalized variation of the ion peak's amplitude and CV position. 

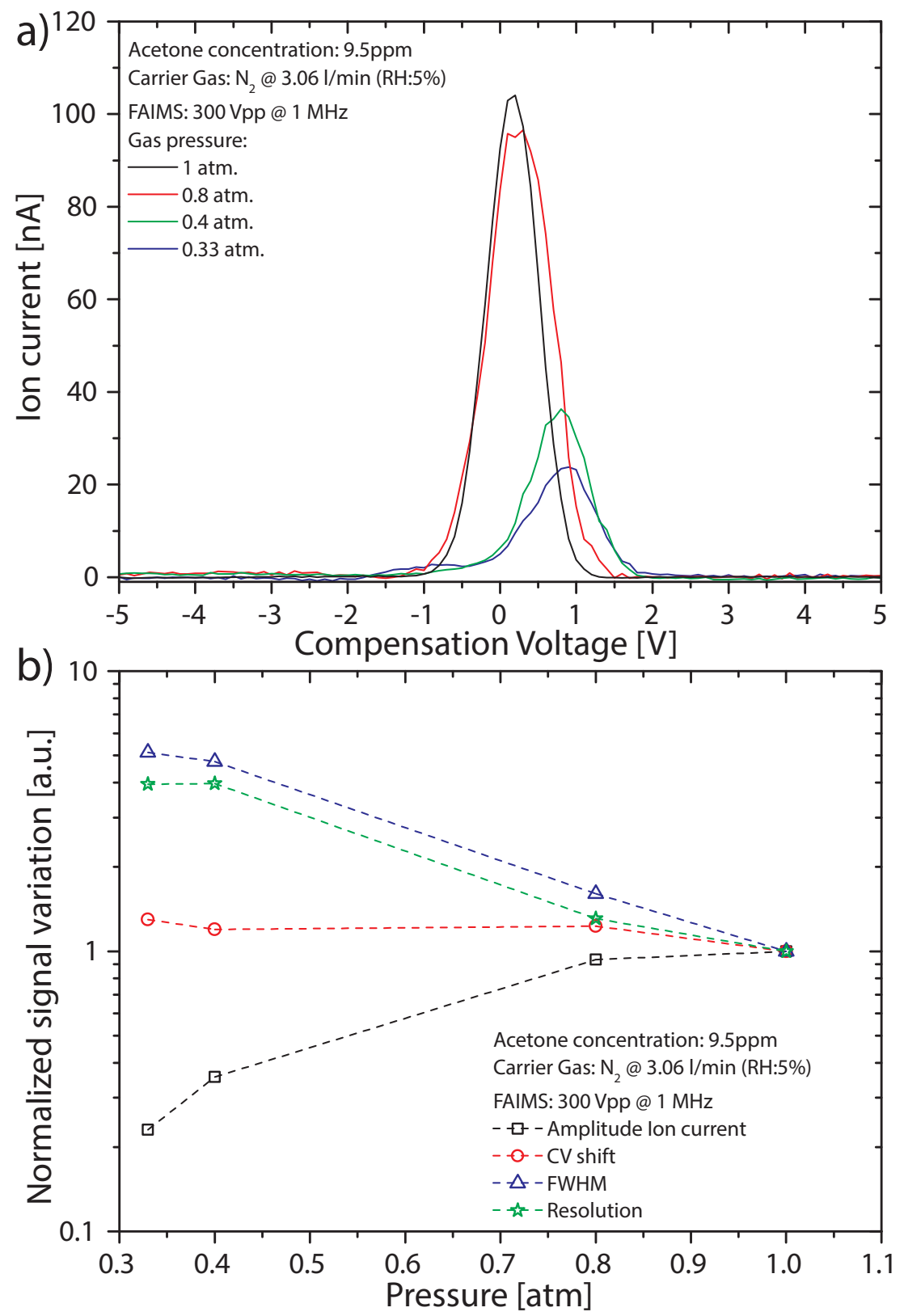

Figure 4.15: Effects of the carrier gas pressure on the FAIMS signal. (a) Detected spectra of 9.5 ppm of acetone at different pressures (1 scan per plot). Nitrogen was used as carrier gas with a flowrate of $3.06 \mathrm{l} / \mathrm{min}$ and relative humidity of 5\%. The pressure was changed from 0.33 to 1 atm. The separation voltage was set to $300 V_{P P}$ with a $33 \%$ duty cycle. (b) Normalized variation of the detected spectra's characteristic parameters. The dashed lines between the points are only visual guides, not fitted lines or theoretical modeling of the physical behavior.

\subsubsection{Effects of the carrier gas flow rate on the ion separation}

As explained in section 4.1.3, increasing the carrier gas flow rate reduces the ion losses, which improves the sensitivity. It also decreases the effect of the ion filter, which leads to a broadening of the ion peaks and a slight reduction on the CV shift. Figures 4.16 to 4.18 show the detected 
acetone spectra at different carrier gas flowrates with low and high humidity levels. The analyte flow was set to $1.2 \times 10^{16}$ acetone molecules per second and the nitrogen flowrate was varied from 2.1 to 14.3 liters per minute.
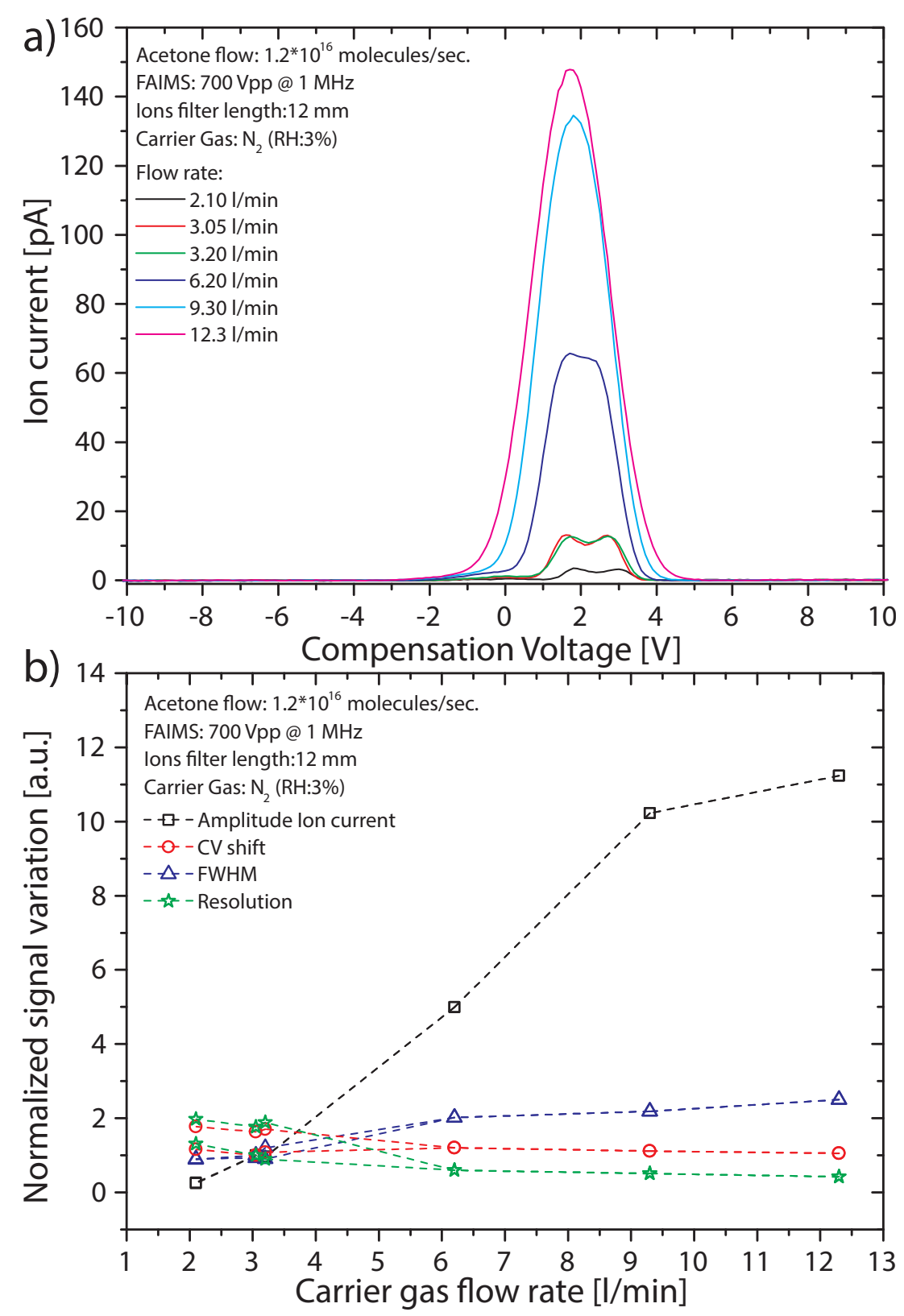

Figure 4.16: Effects of the carrier gas flow rate on detected acetone spectra (a). The analyte flow is constant for all measurements: $1.2 \times 10^{16}$ acetone molecules per second (10 scans per plot). Nitrogen was used as carrier gas with flow rates from 2.1 to $12.3 \mathrm{l} /$ min and relative humidity of 5\%. The separation voltage was set to $700 V_{P P}$ at $1 \mathrm{MHz}$ and 33\% duty cycle. (b) Normalized variation of the detected spectra's characteristic parameters. The dashed lines between the points are only visual guides, not fitted lines or theoretical modeling of the physical behavior. 
At low humidity levels, the signal amplitude increased by more than one order of magnitude along the whole flow range and the FWHM increased about 2.5 times. The CV shift of the main ion peak remained relatively constant. However, when the flow rate increased, the secondary ion peak started fuse with the main one. Therefore, the system resolution decreases about four times between 2 to $6 \mathrm{l} / \mathrm{min}$ flow rates. At higher flow rates, the resolution was dominated by the FWHM variation. Thus, it continued decreasing but at a slower rate. In Figure 4.17, the detected spectra have been normalized to allow a clear observation of the ion peaks' changes in position and shape.

At high humidity levels (60\%), the signal amplitude increased almost 50 times in the measured flow range. The peak's FWHM increased around 50\% while the CV shift remained almost constant. Therefore, the FWHM dominated the FAIMS resolution variation. This results are similar to the ones reported in literature $[5,6,50]$.

The changes in the gas pressure and flow regime inside the ion channel at different flow rates may be some of the factors causing the CV shift and the peak's shape variations. Figure 4.19 shows the differential pressure measured between the start and the end of the ion channel. The gas flow regime start changing from laminar to turbulent at about 6 liters per minute, which leads to an increase in the plot's slope. However, a more detailed study must be done to fully understand these phenomena.

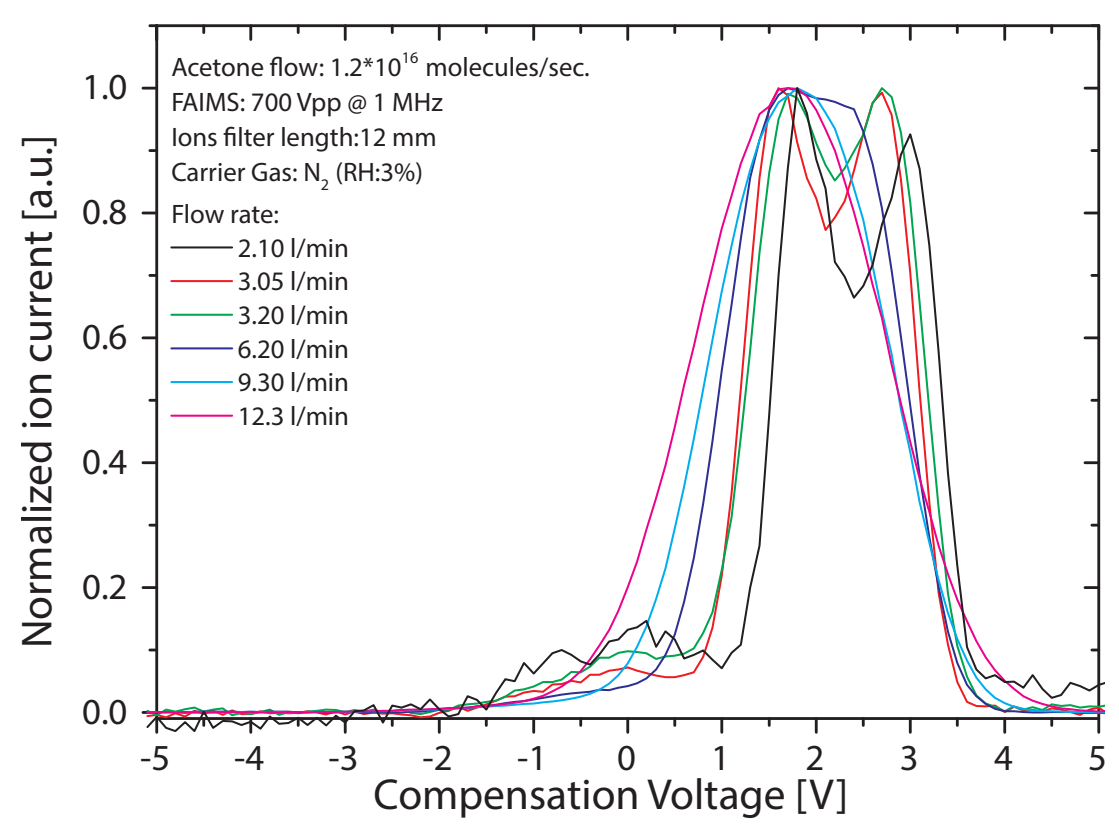

Figure 4.17: Normalized acetone spectra taken at carrier gas flow rates from 2.1 to $14.3 \mathrm{l} / \mathrm{min}$. The position of the main ion peak remains relatively constant at different flow rates. Nevertheless, the CV shift the secondary ion peak decreases with the flow, until it merges with the main peak. 

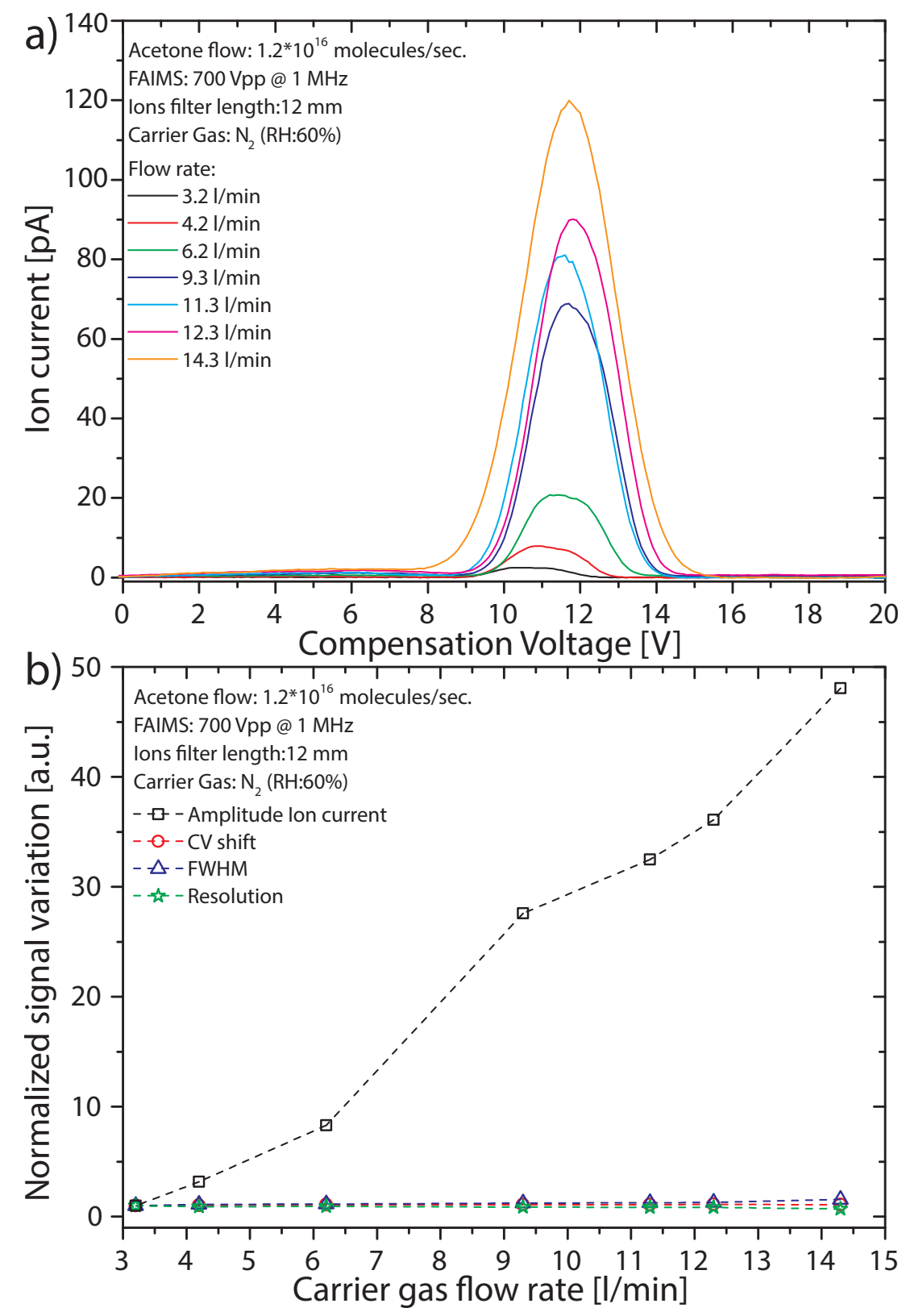

Figure 4.18: Effects of the carrier gas flow rate on detected acetone spectra (a). The analyte flow is constant for all measurements: $1.2 \times 10^{16}$ acetone molecules per second (10 scans per plot). Nitrogen was used as carrier gas with flow rates from 2.1 to $14.3 \mathrm{l} / \mathrm{min}$ and relative humidity of $60 \%$. The separation voltage was set to $700 V_{P P}$ at $1 \mathrm{MHz}$ and $33 \%$ duty cycle. (b) Normalized variation of the detected spectra's characteristic parameters. The dashed lines between the points are only visual guides, not fitted lines or theoretical modeling of the physical behavior. 


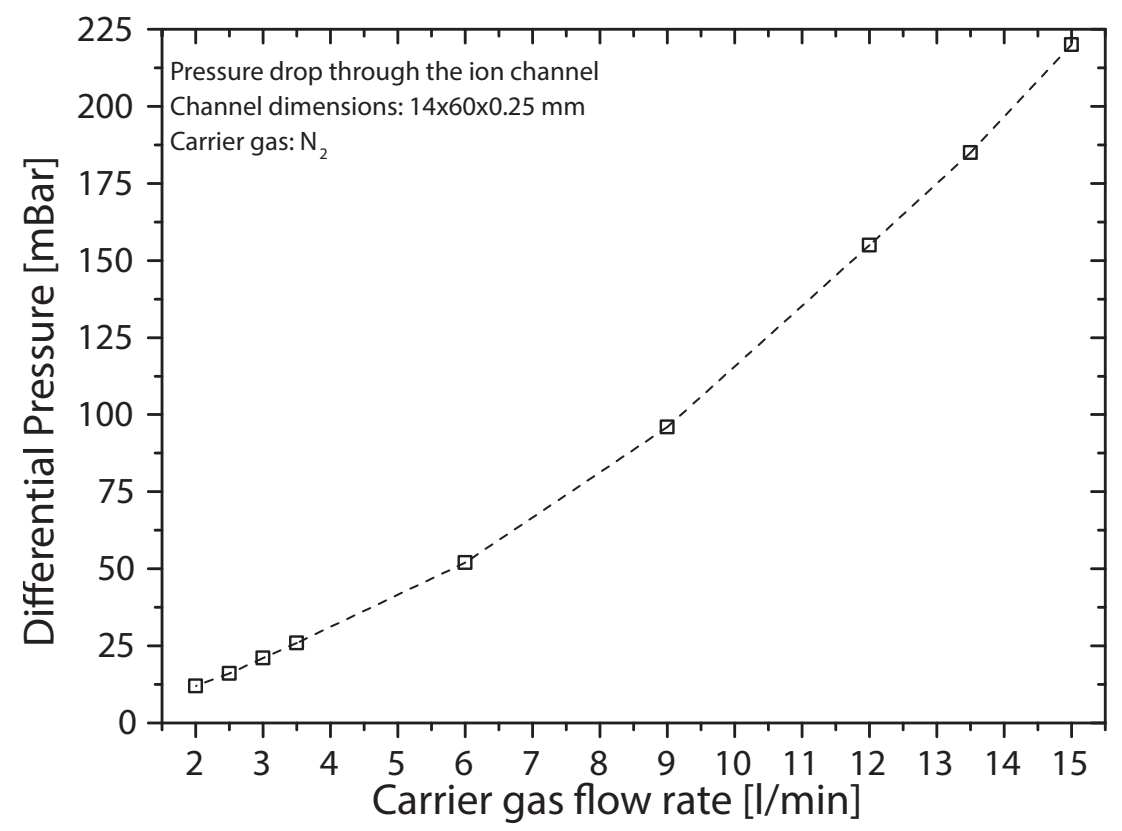

Figure 4.19: Measured differential pressure, between the ions' channel input and output, at gas flow rates from 2.1 to $15 \mathrm{l} / \mathrm{min}$. The dashed lines between the points are only visual guides, not fitted lines or theoretical modeling of the physical behavior.

\subsubsection{Effects of the filter size on the ion separation}

Decreasing the size of the ion filter electrodes gives a reduction in the distance between the ionization region and the ions' detector. This reduces the ions' traveling time inside the ion channel, which decreases the amount of ion losses without affecting the carrier gas properties, e.g. velocity, pressure, flow regime. With the aim of understanding all the experimental effects of decreasing the ion filter length, four FAIMS cores were fabricated with 5, 8, 12 and $15 \mathrm{~mm}$ long ion filter electrodes. Pictures and schematics (including dimensions) of the fabricated electrode plates are shown in Figures 3.4, 4.20 and Appendix B. In order to minimize any external factors, it was decided to keep all the other parameters constant, e.g. the carrier gas flow rate $(3.06 \mathrm{l} / \mathrm{min})$, the ion channel dimensions $\left(14 \times 60 \times 0.25 \mathrm{~mm}^{3}\right)$, the distance between the ionization region and the detector ( $32 \mathrm{~mm}$ from center to center), etc. Figures 4.21 to $4.23 \mathrm{show}$ the different FAIMS spectra measured with the fabricated cores. Two sets of measurements were performed at low (Figures 4.21 and 4.22) and high (Figure 4.23) humidity levels.

Figure 4.21 shows the detected acetone spectra with a filter region length of 5,8 and $12 \mathrm{~mm}$ and filtering voltages of $0 \mathrm{Vpp}$ (a), $300 \mathrm{Vpp}$ (b), $500 \mathrm{Vpp}$ (c) and $700 \mathrm{Vpp}$ (d). The waveform frequency was set to $1 \mathrm{MHz}$ with $33 \%$ duty cycle. As the separation voltage increases, the effects of the different filter sizes on the detected ion spectra become more noticeable. While the total amplitude variation at $0 \mathrm{Vpp}$ is only $10 \%$, at $700 \mathrm{Vpp}$ it reaches almost $400 \%$. Also, the secondary peaks, at the left and right sides of the main ions' peak, are more distinguishable at higher voltages and longer ion filters. Conversely, the resolution variation of the main ions' peak only seems to be dependent on the filter length and not on the separation voltage. When the filter length is reduced from 12 to $8 \mathrm{~mm}$, the CV shift and the FWHM change, whilst when 
it is reduced from 8 to $6 \mathrm{~mm}$ only the FWHM changes. Therefore, the resolution decrease is stronger in the first case than in the second.

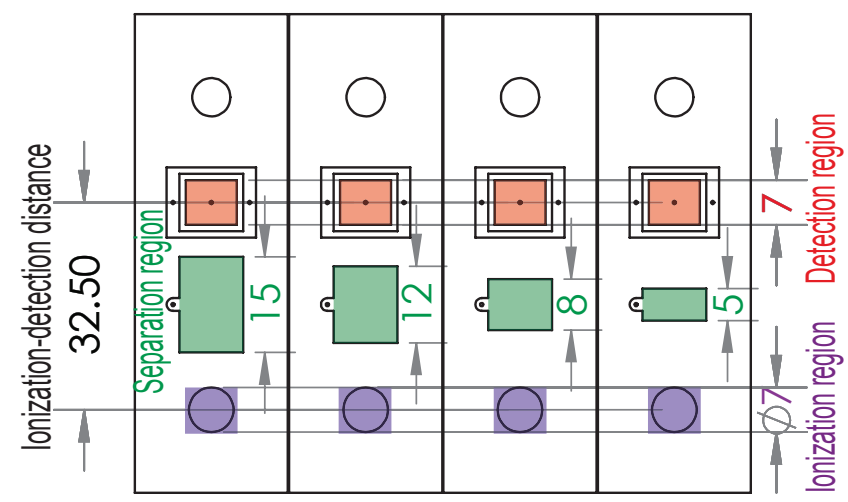

Figure 4.20: Schematics of the implemented electrode plates with different ion filter (green) lengths and constant distance between the ionization region (purple) and the ion detector (red). All dimensions are in $\mathrm{mm}$.
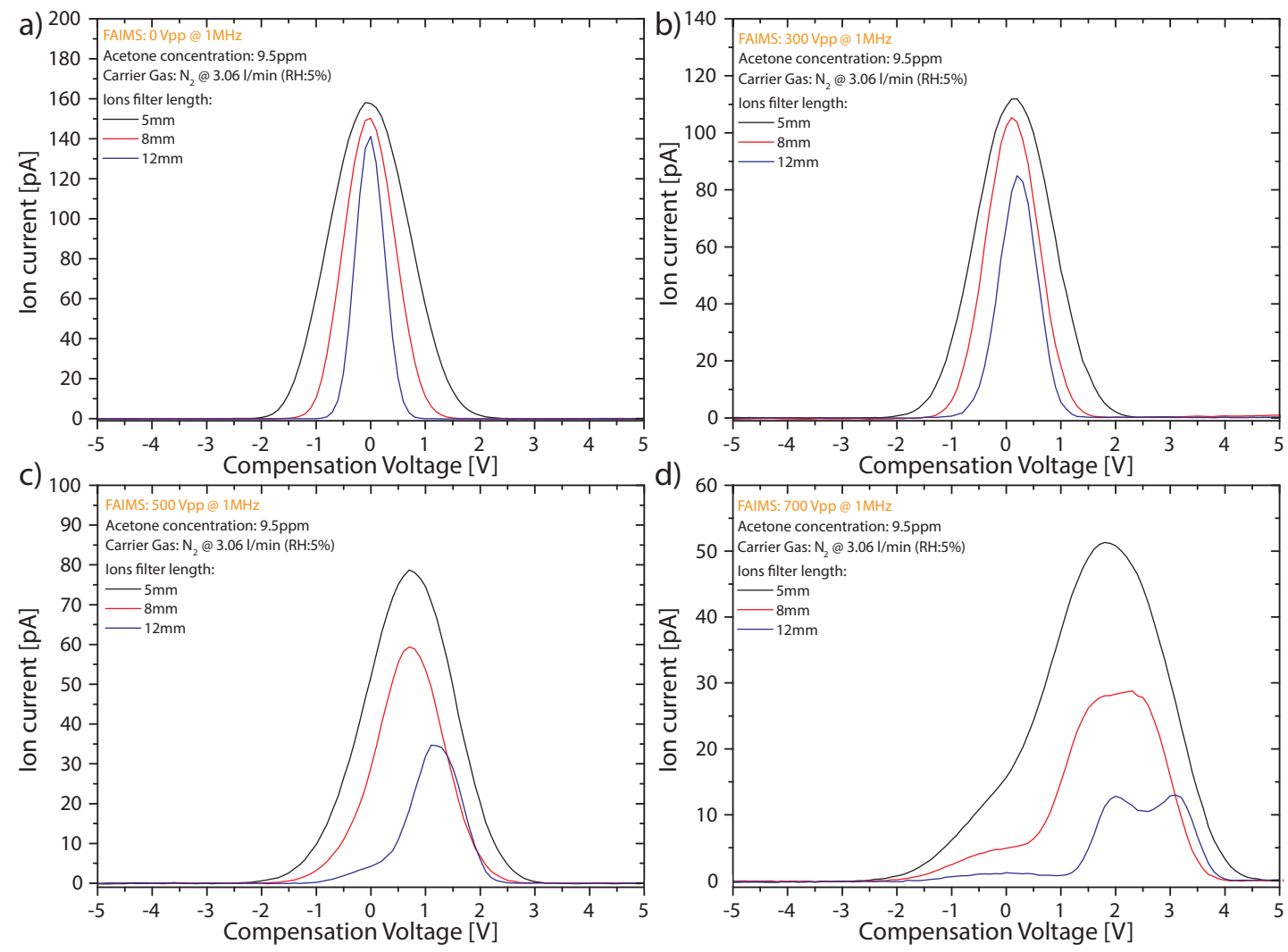

Figure 4.21: Effects of the ion filter length on detected acetone (9.5 ppm) spectra at different separation voltages, i.e. $0 \mathrm{~V}(\mathrm{a}), 300 V_{P P}(\mathrm{~b}), 500 V_{P P}(c)$ and $700 V_{P P}(d)$. The nitrogen flow rate was set to $3.06 \mathrm{l} / \mathrm{min}$ with $5 \%$ relative humidity. The applied $H V$ waveform had a frequency of $1 \mathrm{MHz}$ and $33 \%$ duty cycle. Each curve is the average of 10 measurements under the same conditions. 
At higher humidity levels (75\%), the effects of the filter size on the acetone spectra are similar as the ones observed at low humidity. However, the split between the main and the right peaks only occurs at the $15 \mathrm{~mm}$ long filter. Also, the left side peak is more noticeable, especially at short filter lengths, and the CV shift of the main peak is no longer constant between 5 and $8 \mathrm{~mm}$ filters. Figure 21 shows the detected acetone spectra using filters from 5 to $15 \mathrm{~mm}$ long and a filtering voltage of $700 \mathrm{VPP}_{\mathrm{PP}}$ at $1 \mathrm{MHz}$ and $33 \%$ duty cycle. The measurements were normalized to allow a clear observation of the ion peaks' changes in position and shape.

In summary, reducing the ions filter's length also reduces the time the ions spend in the filtering region. This leads to a behavior analogous to the one caused by increasing the carrier gas flow rate in section 4.2.6. The increase in the signal's amplitude with shorter filter electrodes was much lower than the one observed in the flow rate experiments. However, it must be taken into account that the total distance between the ionization region and the ion detector was kept constant, as well as the carrier gas flowrate. Thus, a higher improvement in the systems sensitivity can be expected if the total ion path is also reduced with the filter electrodes. Unfortunately, the effects of reducing the ion filter's length on the system's resolution (CVshift/FWHM) are much stronger than the ones caused by increasing the carrier gas flow rate, i.e. the reduction of the CV shift and the widening of the peaks' FWHM.
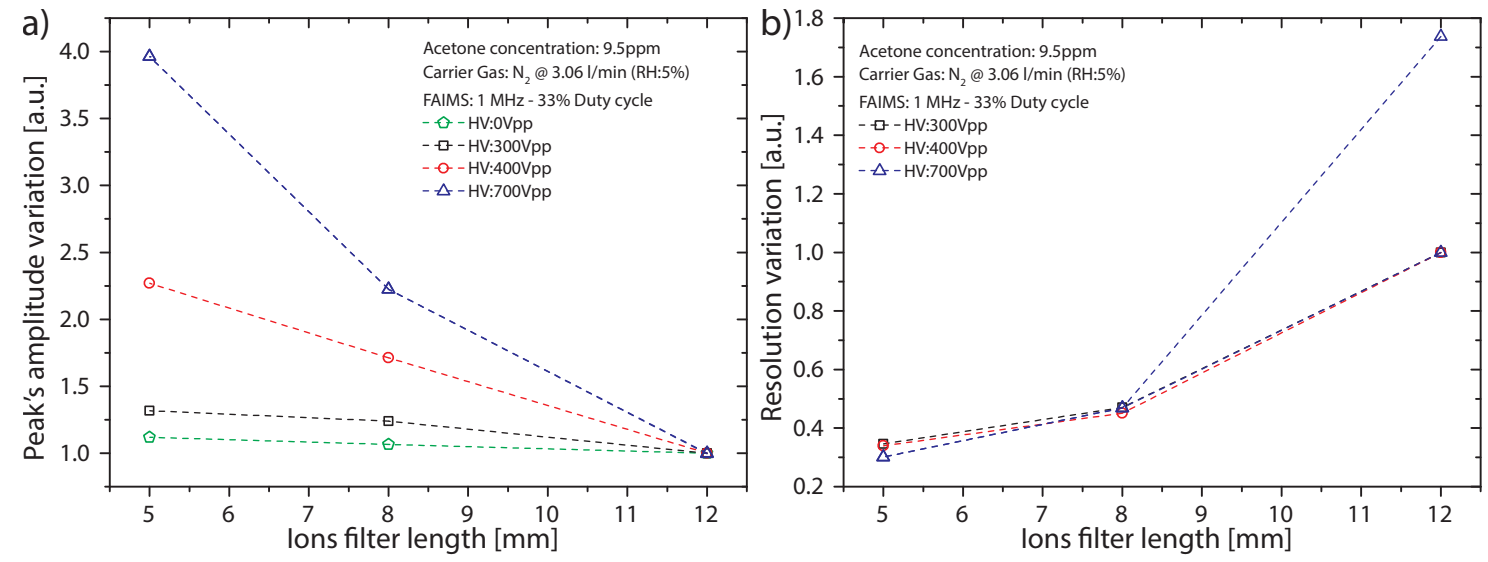

Figure 4.22: Normalized variation of acetone spectra's amplitude and resolution at different ion filter lengths and separation voltages. The length of the ion filter was varied from 12 to 5 $\mathrm{mm}$. The nitrogen flow rate was set to $3.06 \mathrm{l} / \mathrm{min}$ with 5\% relative humidity. The applied $\mathrm{HV}$ waveform was changed from 0 to $700 V_{P P}$ with a frequency of $1 \mathrm{MHz}$ and $33 \%$ duty cycle. Each point is the average of 10 measurements under the same conditions. The dashed lines between the points are only visual guides, not fitted lines or theoretical modeling of the physical behavior.

Finally, since the filter electrodes were centered between the ionization region and the ion detector, the distance between the filter and the detector increases when the length of the filter electrodes is reduced. This allows the analysis of the effect of a larger inter-electrode distance in the amplifier's noise spectral density. Figure 4.24 shows the noise spectral density detected at inter-electrode distances from 5 to $10 \mathrm{~mm}$ and a reference measurement with the ion filter off. The $\mathrm{HV}$ signal was kept constant at $700 \mathrm{~V}_{\mathrm{PP}}, 1 \mathrm{MHz}$ and $33 \%$ duty cycle during all the measurements. As expected, the coupled 1/f noise levels slightly increase at lower 
filter-detector distance. At frequencies higher than $2 \mathrm{~Hz}$, the noise spectral density is governed by the thermal noise of the amplifier's resistor. Therefore, after $2 \mathrm{~Hz}$, the ion filter does not have any influence on the coupled noise levels.
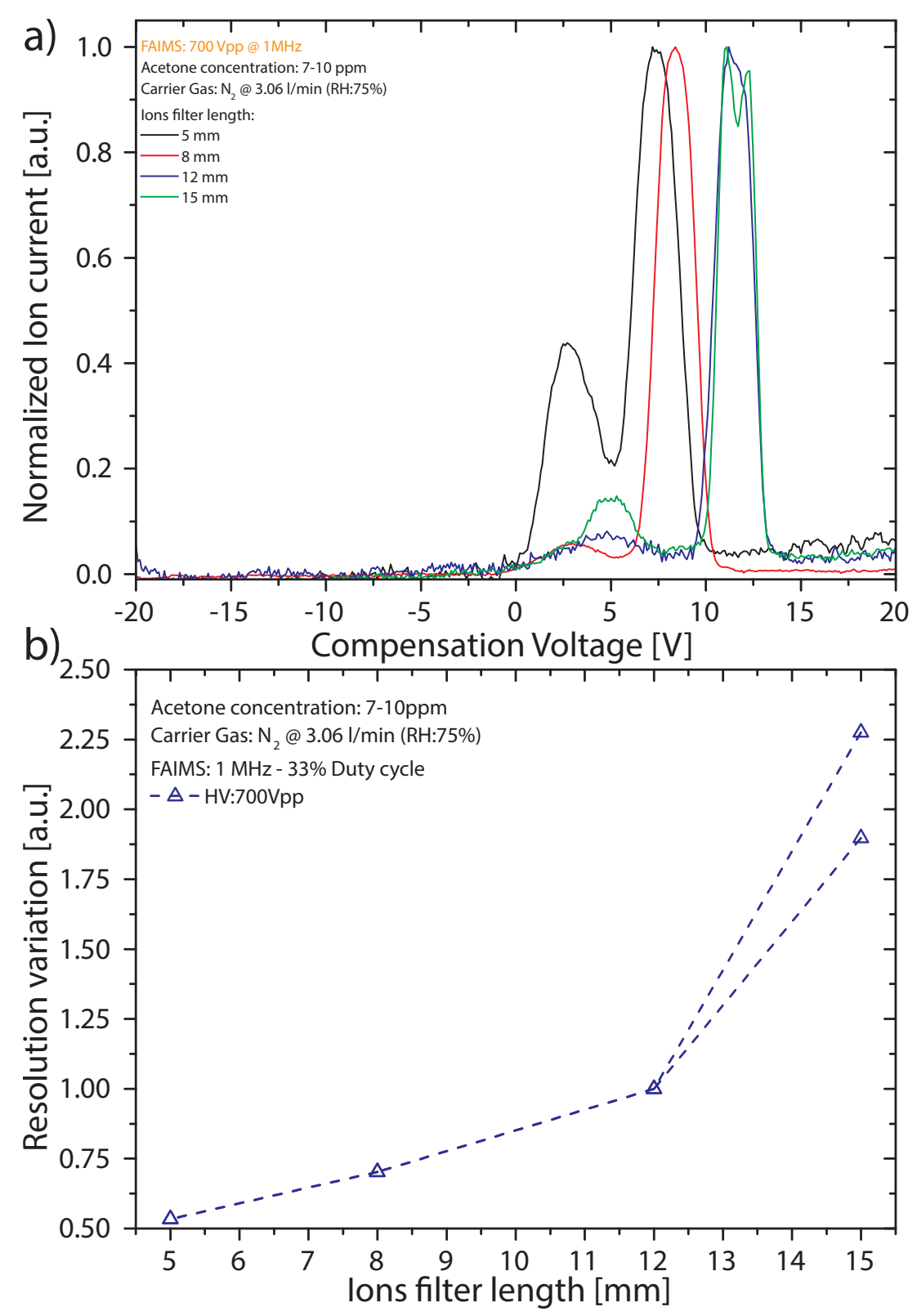

Figure 4.23: Effects of the ion filter length on detected acetone spectra. The length of the ion filter was varied from 15 to $5 \mathrm{~mm}$. The nitrogen flow rate was set to $3.06 \mathrm{l} / \mathrm{min}$ with $75 \%$ relative humidity. The ion filter was performed with a $700 V_{P P}$ waveform with a frequency of $1 \mathrm{MHz}$ and $33 \%$ duty cycle. Each curve is the average of 10 measurements under the same conditions. The dashed lines between the points are only visual guides, not fitted lines or theoretical modeling of the physical behavior. 


\subsection{Ion detector}

The ion detector is formed by two parallel electrodes, each surrounded by a ground electrode, a shown in Figure 3.4. One of the electrodes is connected to a voltage source and the other to the low noise current amplifier. The electric field in the detection region deflects the ions towards the detector electrode generating a small ion current (typically 0 to $1 \mathrm{nA}$ ). This current is amplified and processed to produce a clear ion spectrum signal. The main purpose of this section is to determine the best detection conditions for a high ion capture rate with minimum disruption to the electric field inside the ion channel.

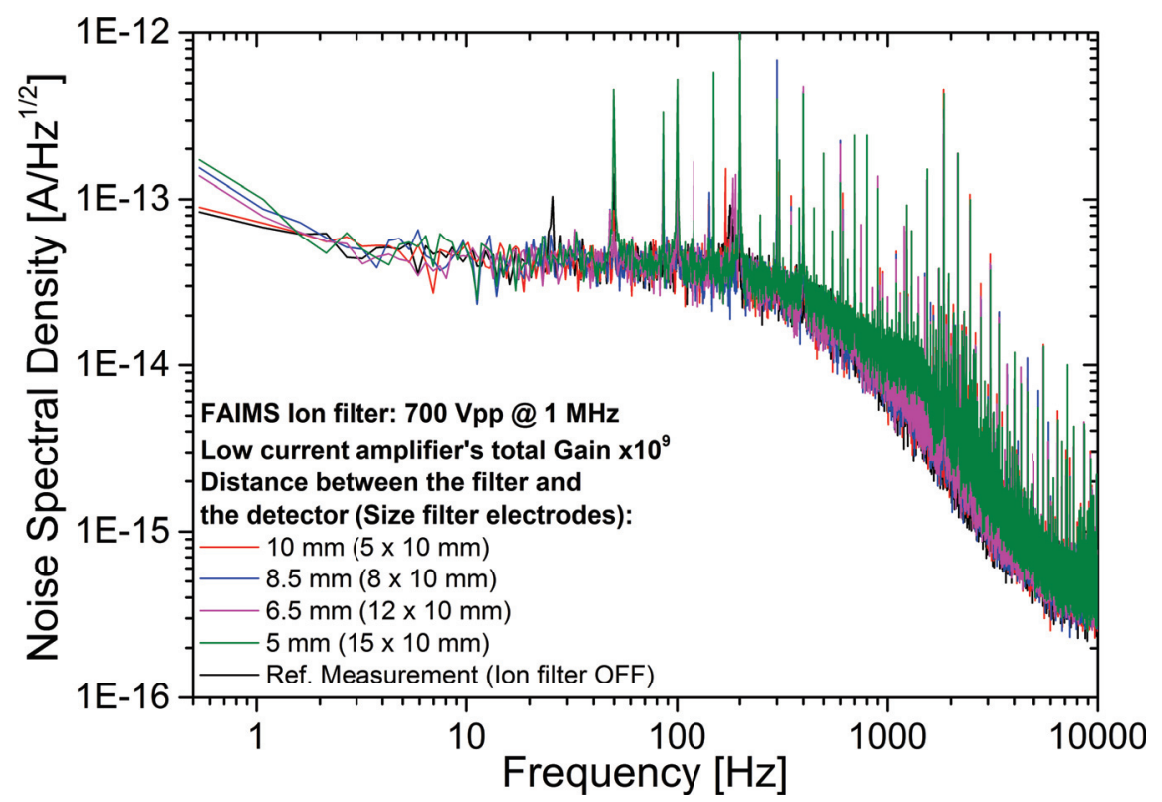

Figure 4.24: Noise spectral density from the low noise current amplifier measured at filterdetector distances from 5 to $10 \mathrm{~mm}$. The ion filter waveform was set to $700 V_{P P}$ at $1 \mathrm{MHz}$ and $33 \%$ duty cycle. An additional measurement (black curve) with the ion filter off is presented as reference. Each curve is the average of 10 measurements under the same conditions.

\subsubsection{Effect of the deflection voltage on the ion capture rate}

The defection voltage is in charge of generating the electric field that directs the ions towards the detector electrode. If this voltage is too low, the ions will be carried away by the gas flow and will not be detected. Several measurements were taken in order to determine the behavior of the detected signal's amplitude at different deflection voltages. The measurements were performed at low $(3.05 \mathrm{l} / \mathrm{min})$ and high $(11 \mathrm{l} / \mathrm{min})$ carrier gas flowrates, shown in Figure 4.25, with the ion filtering OFF $(\mathrm{a}, \mathrm{c})$ and $\mathrm{ON}(\mathrm{b}, \mathrm{d})$.

The experimental results showed a rapid increase in the amplitude of the detected ion peaks when the deflection voltage is applied. At about $5 \mathrm{~V}$, the signal amplitude saturates. If the deflection voltage is increased further, the signal's amplitude starts to decrease. This may be caused by the electric field disturbances between the detector electrodes and the filter and ground electrodes. Since ions at higher gas flowrates cross faster the boundary regions between the electrodes, they are less affected by the field irregularities. Therefore, the signal 
amplitude reduction at high DVs is less pronounced at higher gas flowrates. Besides the change in the peaks' amplitude, no other effect was observed in the ions spectra.
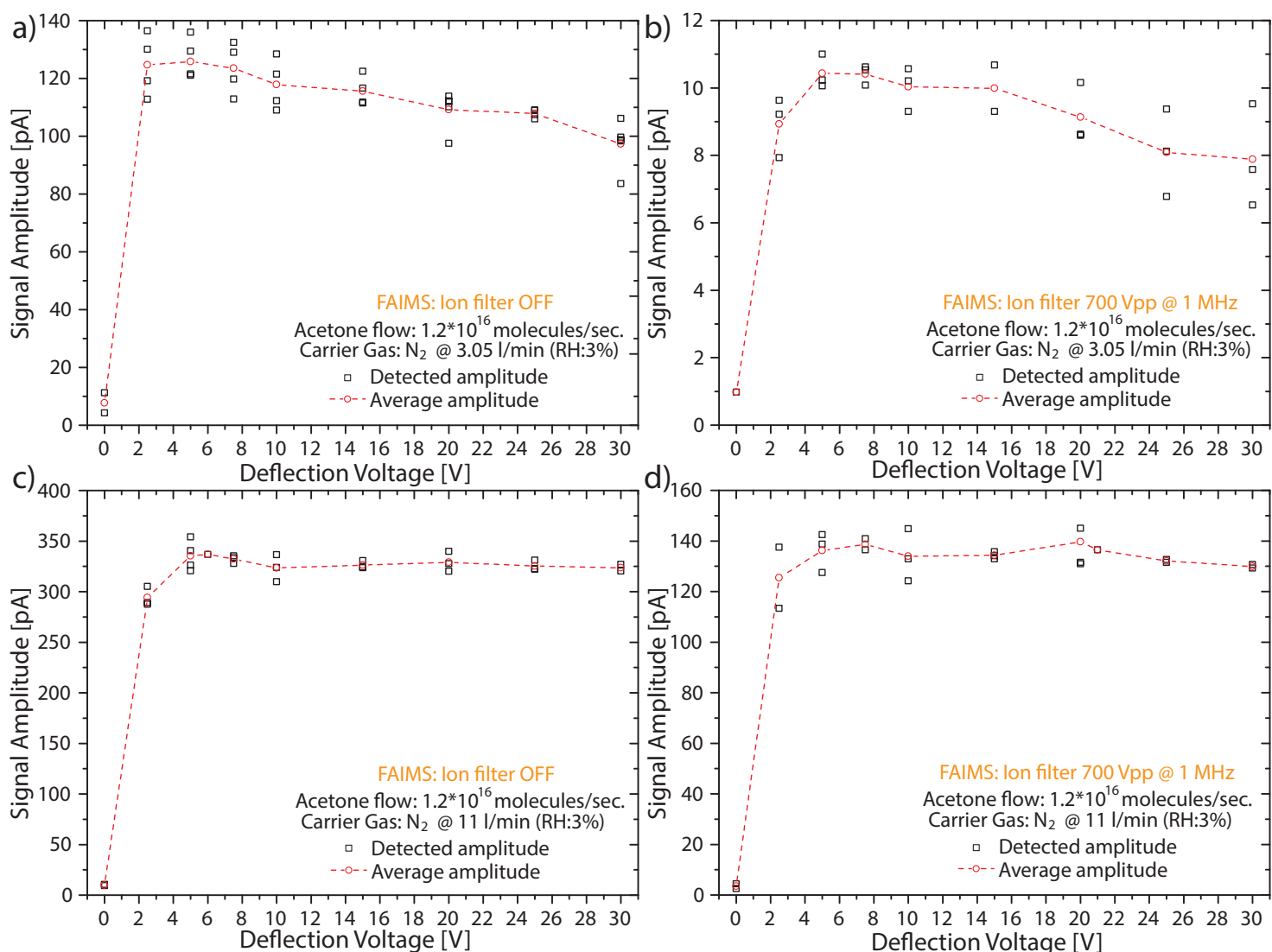

Figure 4.25: Variation of the detected acetone ion peak at different deflection voltage levels. The analyte flow was kept constant to $1.2 \times 10^{16}$ molecules per second. The nitrogen flow was set to $3.05(a, b)$ and $11(c, d) l /$ min with a humidity level of $3 \%$. The measurements in $(a, c)$ were taken without the ion filter's $H V$ signal while the one in $(b, d)$ were taken with a filter voltage of 700 $V_{P P}$ at $1 \mathrm{MHz}$ and 33\% duty cycle. The black symbols represent the experimental data and the red ones the average value. The dashed lines between the points are only visual guides, not fitted lines or theoretical modeling of the physical behavior.

\subsubsection{Lock-in detection}

In order to improve the measurements' SNR, a setup for the synchronous detection of the ion current was implemented (Figure 3.16). The effect of the modulation/demodulation process in the detected ions' spectra was characterized by taking several measurements at different modulation signal's amplitudes and frequencies. The experiments were performed at room temperature and atmospheric pressure using nitrogen as the carrier gas and acetone as the analyte. The analyte flow was set to $2.5 \times 10^{15}$ acetone molecules per second. The measurements were taken at a sampling rate of $12 \mathrm{kHz}$ with an averaging time of 1 second per point. The compensation voltage was increased at steps of $0.1 \mathrm{~V} / \mathrm{s}$. All experimental parameters are summarized in appendix D. 
Effects of the modulation signal's amplitude on the ions' spectra
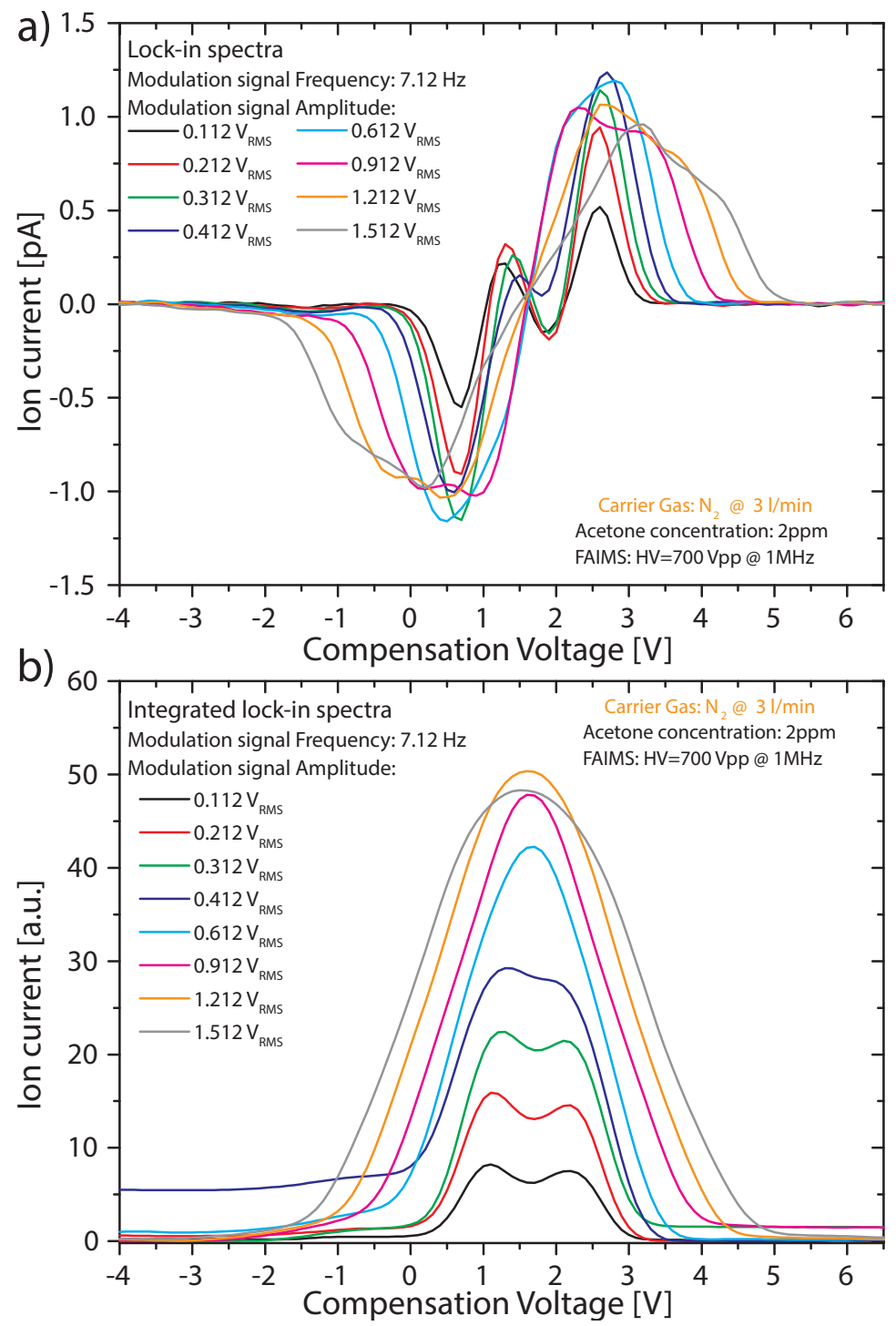

Figure 4.26: Detected acetone spectra using the FAIMS + lock-in amplifier setup at different modulation amplitudes. Plot (a) shows the original signals from the Lock-in amplifier while plot (b) the integrated lock-in spectra. The modulation frequency was set to $7.12 \mathrm{~Hz}$. The carrier gas and the acetone flowrates were kept constant at $3.05 \mathrm{l} / \mathrm{min}$ and $2.5 \times 10^{15}$ molecules/second respectively. The ion filtering was performed at $700 V_{P P}, 1 \mathrm{MHz}$ and 33\% duty cycle. Additional experimental parameters are summarized in Appendix D.

The first characterized parameter was the amplitude of the modulation signal. Figures 4.26 and 4.27 show the detected acetone spectra at modulation amplitudes from 0.112 to $1.512 \mathrm{~V}_{\mathrm{RMS}}$ at low and high modulation frequencies, respectively. The original signals from the Lock-in amplifier are presented in (a) and the integrated lock-in signals in (b). Also, the variations in the ion peaks' amplitude and FWHM are summarized in Figure 4.28. At low modulation levels, 
the ion peak's amplitude increases with the modulation amplitude while the FWHM remains constant. However, at modulation amplitudes higher than $0.312 \mathrm{~V}_{\mathrm{RMS}}$, the detected signal is "over modulated' and, hence, distorted. Therefore, the peak's amplitude saturates, and then starts to decrease, while the FWHM monotonically increases. Consequently, in terms of SNR and resolution, it is better to choose the modulation amplitude just before the FWHM starts to increase, in this case $0.312 \mathrm{~V}_{\mathrm{RMS}}$. The effects of the modulation amplitude on the ion spectra are almost the same at low and high modulation frequencies. Also, the base line noise level was not affected by the variation of the modulation amplitude.
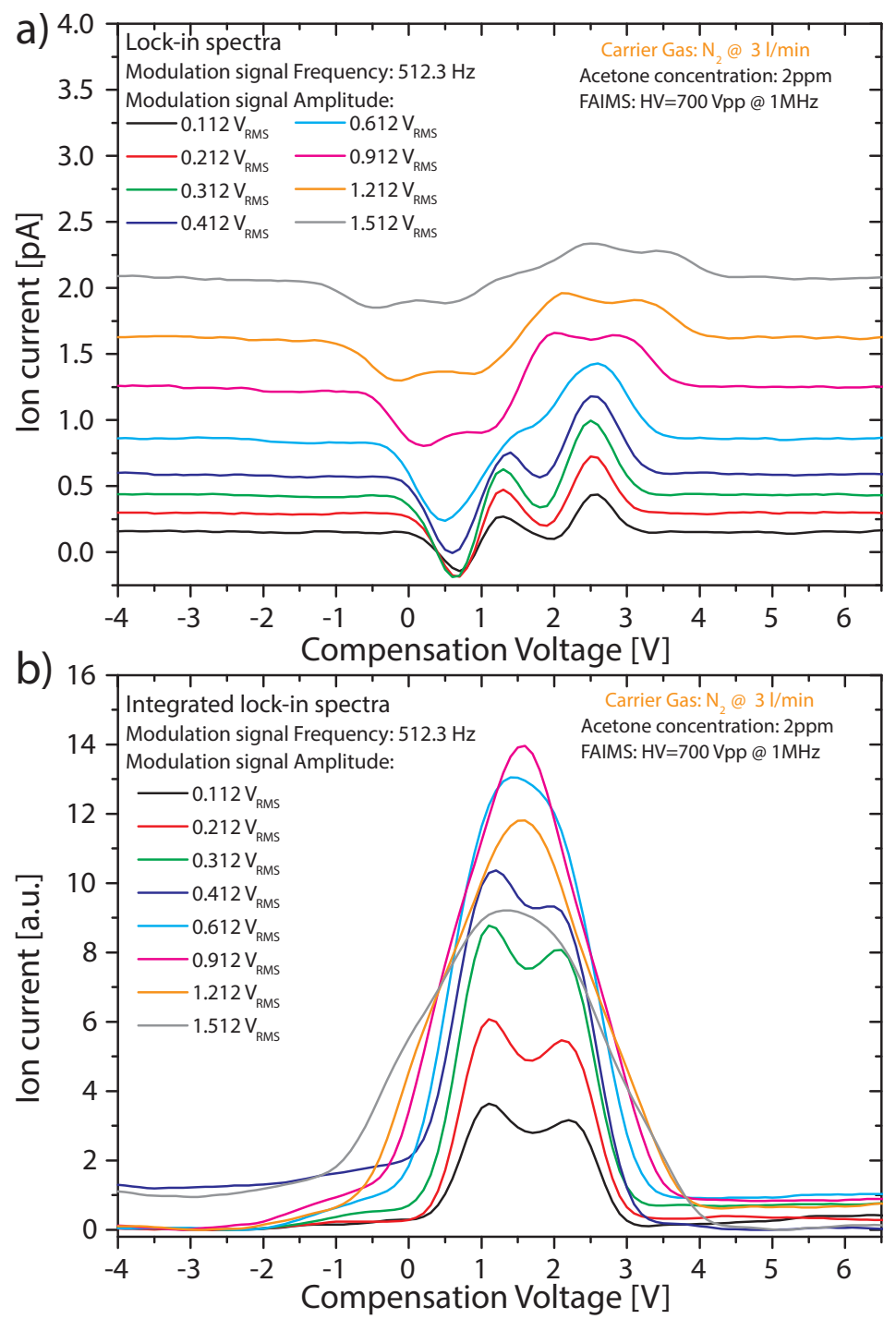

Figure 4.27: Detected acetone spectra using the FAIMS + lock-in amplifier setup at different modulation amplitudes. Plot (a) shows the original signals from the Lock-in amplifier while plot (b) the integrated lock-in spectra. The modulation frequency was set to $512.3 \mathrm{~Hz}$. The carrier gas and the acetone flowrates were kept constant at $3.05 \mathrm{l} / \mathrm{min}$ and $2.5 \times 10^{15}$ molecules/second respectively. The ion filtering was performed at $700 V_{P P}, 1 \mathrm{MHz}$ and $33 \%$ duty cycle. Additional experimental parameters are summarized in Appendix D. 

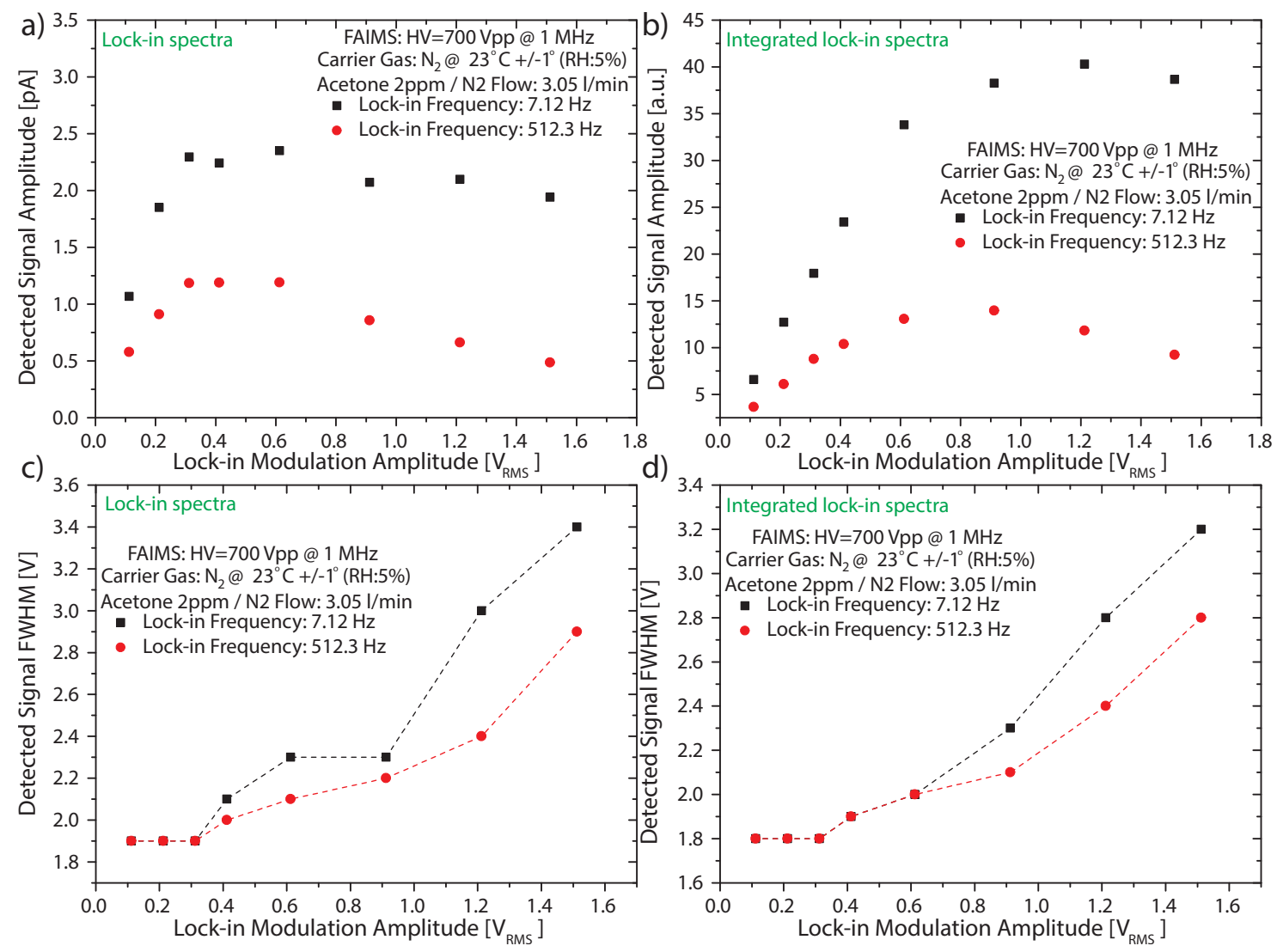

Figure 4.28: Variation of the detected signal's amplitude $(a, b)$ and FWHM $(c, d)$ at different modulation amplitudes with low (black) and high (red) modulation frequency: The left side plots $(a, c)$ show the behavior of the original signals from the Lock-in amplifier while the right side plots $(b, d)$ show the behavior of integrated spectra. The carrier gas and the acetone flowrates were kept constant at $3.05 \mathrm{l} / \mathrm{min}$ and $2.5 \times 10^{15}$ molecules/second respectively. The ion filtering was performed at $700 V_{P P}, 1 \mathrm{MHz}$ and $33 \%$ duty cycle. Additional experimental parameters are summarized in Appendix D. The dashed lines between the points are only visual guides, not fitted lines or theoretical modeling of the physical behavior.

\section{Effects of the modulation signal's frequency on the ions' spectra}

Once the optimal modulation amplitude has been defined, the next step is to determine the best modulation frequency. Several measurements were taken with modulation frequencies from 1 to $812 \mathrm{~Hz}$. Figure 4.29 shows the detected acetone spectra at different modulation frequencies. In order to evaluate the influence of the ion velocity on the lock-in measurements, two sets of experiments were done at low (Figure 4.29.a) and high (Figure 4.29.b) gas flow rates, i.e. 3.05 and $9.51 / \mathrm{min}$ respectively. Additionally, Figure 4.30 summarize the variations in the ions peak's amplitude (a) and noise levels (b) at different modulation frequencies.

The ion's residence time inside the filter region $\left(\Delta t_{i}\right)$ is about $0.8 \mathrm{~ms}$ at a gas flowrate of $3.06 \mathrm{l} / \mathrm{min}$ and $0.2 \mathrm{~ms}$ at a flowrate of $9.5 \mathrm{l} / \mathrm{min}$. Therefore the expected modulation cutoff frequencies $\left(f_{c-L I}\right)$ are 200 and $800 \mathrm{~Hz}$ respectively $\left(f_{c-L I} \approx \frac{1}{2 \pi \Delta t_{i}}\right)$. 

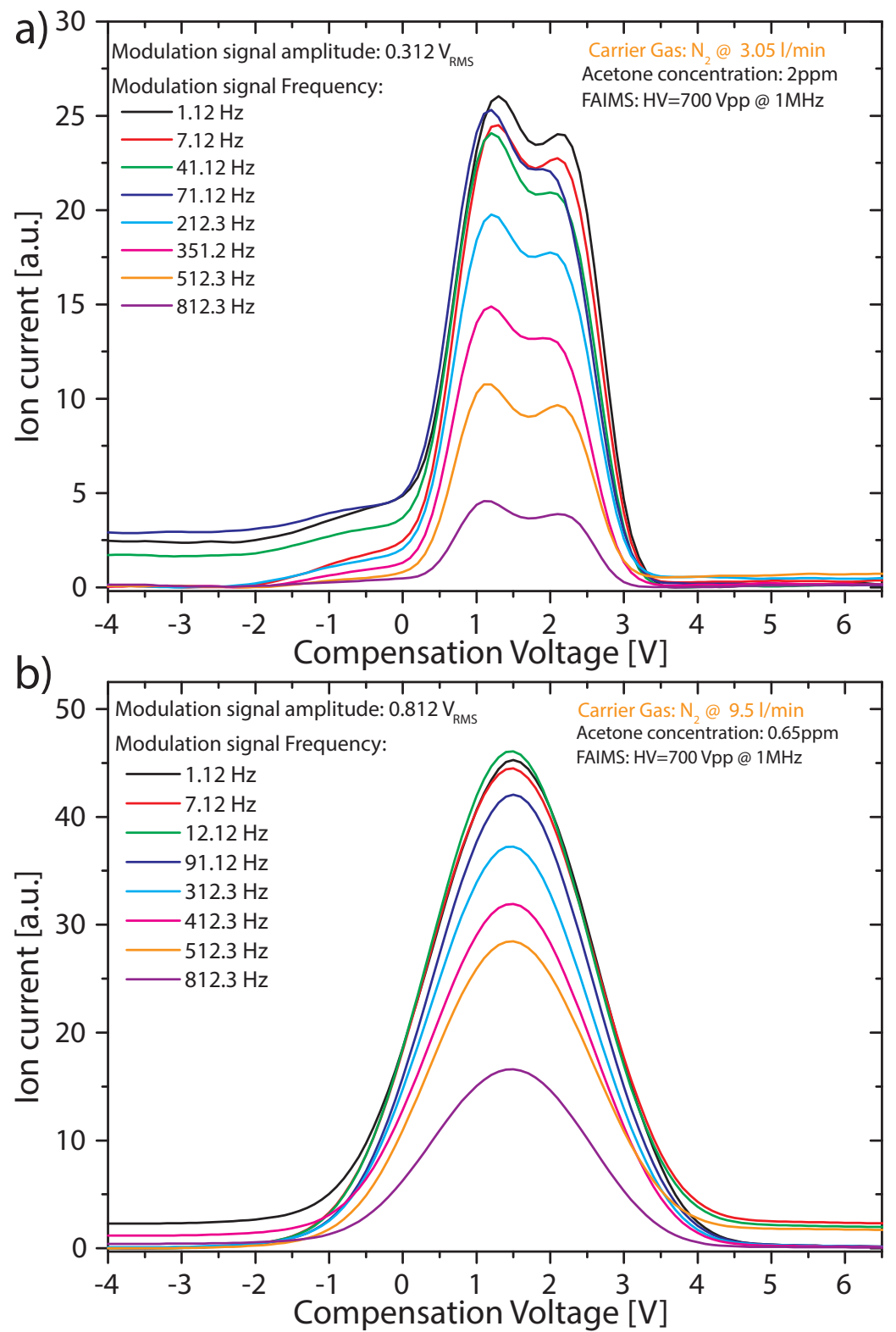

Figure 4.29: Detected acetone spectra using the FAIMS + lock-in amplifier setup at different modulation frequencies. The acetone flowrate was kept constant at $2.5 \times 10^{15}$ molecules/second. Two sets of measurements are presented: the measurements in (a) were taken with a carrier gas flow of $3.05 \mathrm{l} / \mathrm{min}$ while the measurements in (b) at a flowrate of $9.5 \mathrm{l} / \mathrm{min}$. The ion filtering was performed at $700 V_{P P}, 1 \mathrm{MHz}$ and $33 \%$ duty cycle. Additional experimental parameters are summarized in Appendix D. 

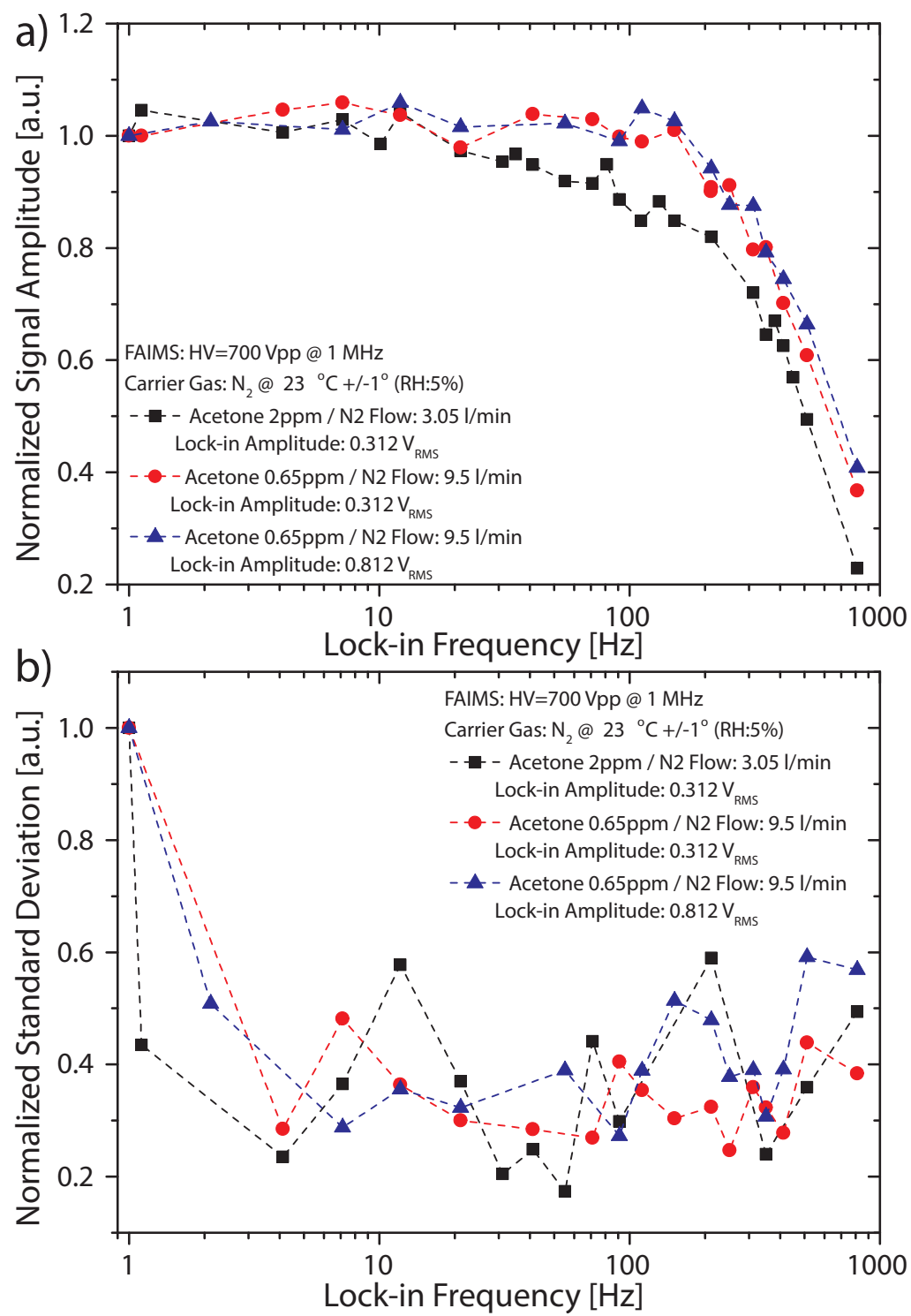

Figure 4.30: Normalized variation versus the modulation frequency of the ion peak's amplitude (a) and FWHM (b) at different gas flowrates, and modulation amplitudes. The acetone flowrate was kept constant at $2.5 \times 10^{15}$ molecules/second. The ion filtering was performed at $700 V_{P P}, 1$ $\mathrm{MHz}$ and 33\% duty cycle. Additional experimental parameters are summarized in Appendix $D$. The dashed lines between the points are only visual guides, not fitted lines or theoretical modeling of the physical behavior.

The measured data (Figure 4.30.a) has a cutoff frequency close to the expected values, i.e. $\sim 400 \mathrm{~Hz}$. However, there was not significant variation in the $f_{c-L I}$ between low and high gas flowrates. The causes of this phenomenon are still unclear.

The detected base line noise levels rapidly decrease when the frequency increases (Figure 4.30.b). At a modulation frequency of about $3 \mathrm{~Hz}$, the noise level reach the minimum value. This behavior agrees with the measured noise spectral density shown in Figure 3.14. Finally, 
from Figures 4.30.a and 4.30.b we can conclude that, as expected, the detected signal's dependencies on the modulation frequency is the same at different modulation amplitudes.

\section{Effects of the modulation/demodulation process on the ion spectra's shape}
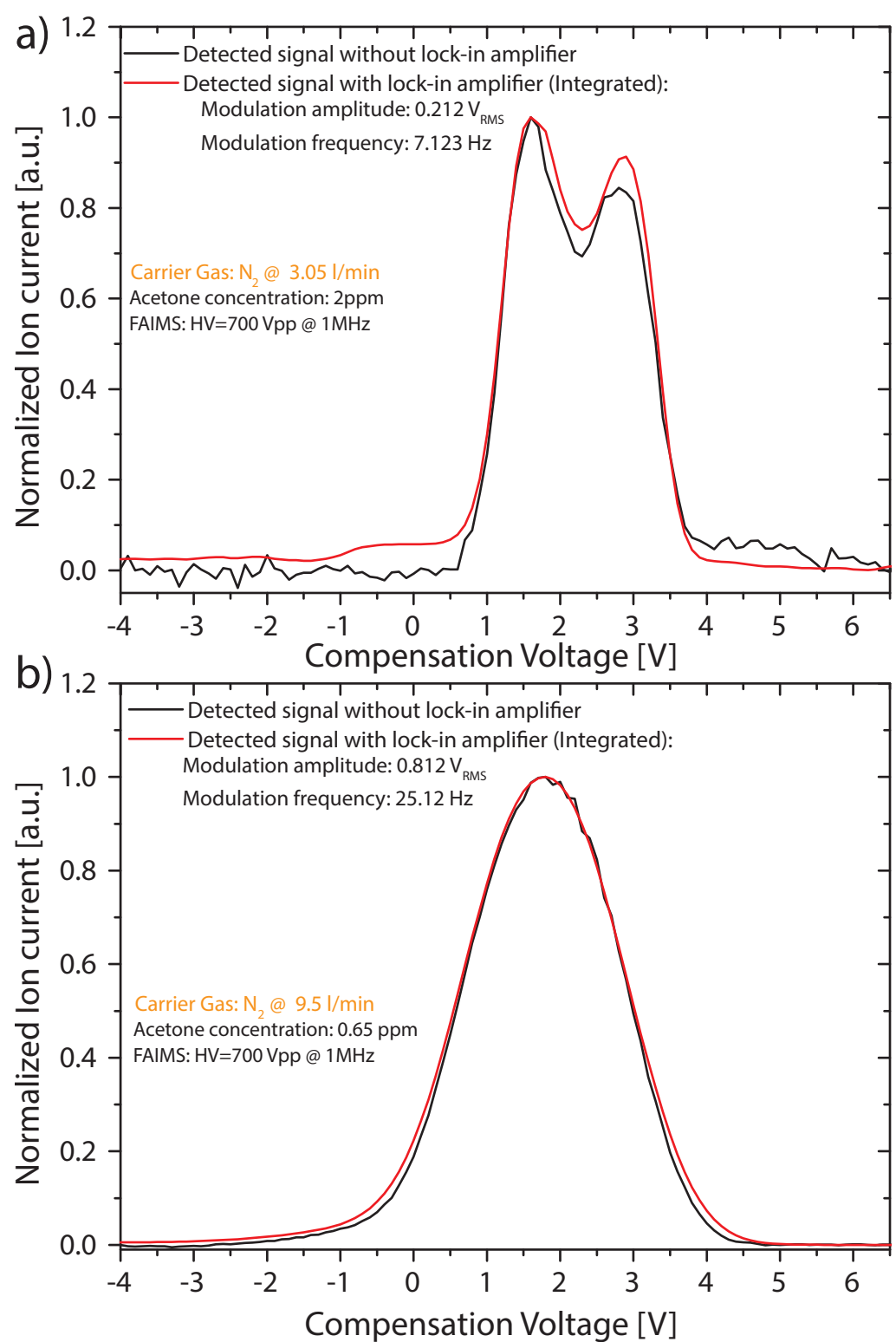

Figure 4.31: Comparison between the detected acetone spectra with and without lock-in detection. (a) The modulation for the measurements with $\mathrm{N}_{2}$ flow of $3.05 \mathrm{l} / \mathrm{min}$ was done at $7.123 \mathrm{~Hz}$ with $0.212 V_{R M S}$ amplitude. (b) The modulation for the measurements with $\mathrm{N}_{2}$ flow of $9.5 \mathrm{l} / \mathrm{min}$ was performed at $25.12 \mathrm{~Hz}$ with $0.812 V_{R M S}$ amplitude.

The comparison among the detected acetone spectra showed similar results between the measurements with and without the lock-in amplifier, as expected. Figure 4.31 presents some examples of the compared acetone spectra at low (a) and high (b) carrier gas flowrates. 


\section{Signal to noise ratio $(\mathrm{SNR})$}

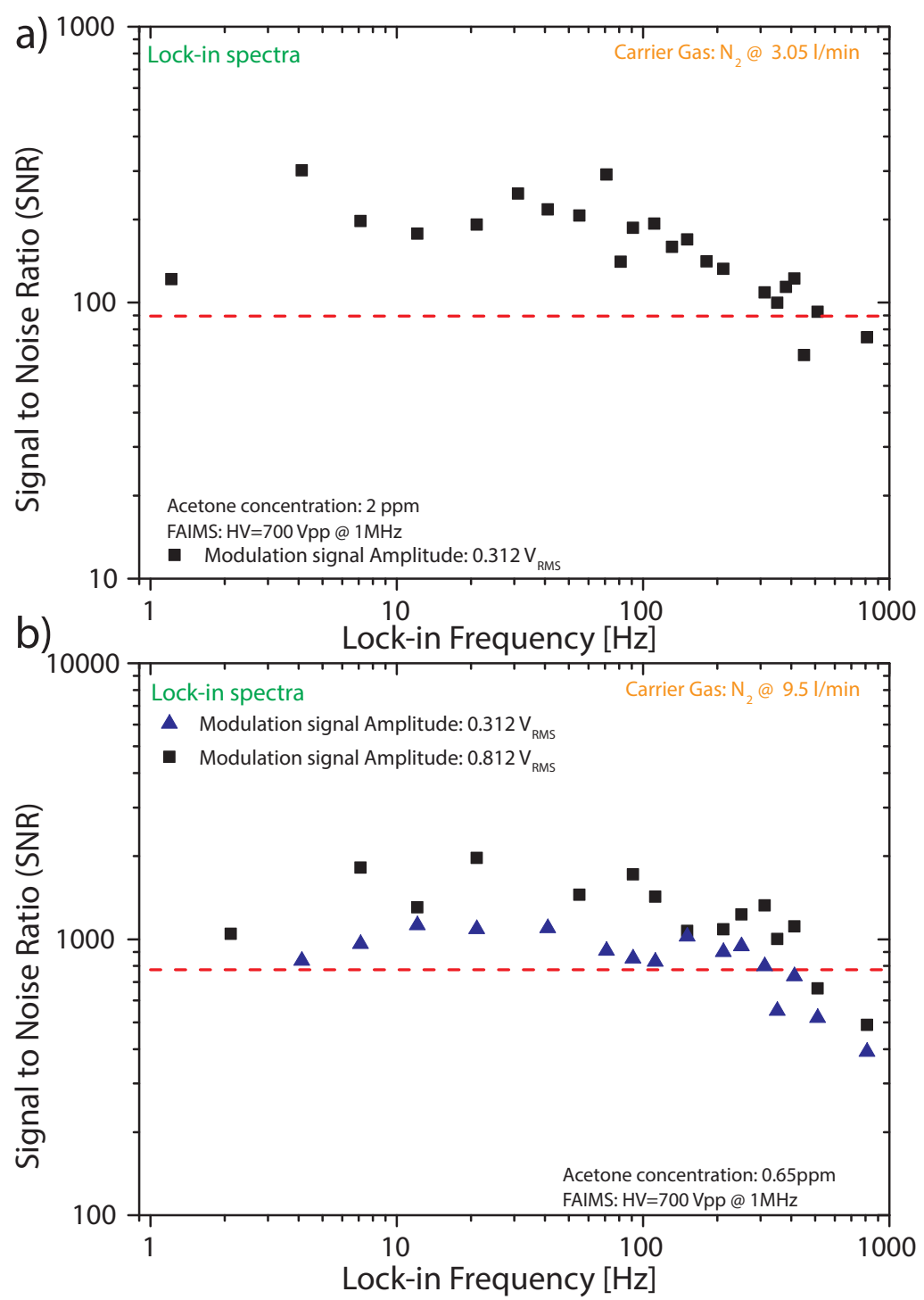

Figure 4.32: SNR variation at different modulation frequencies for gas flows of 3.05 (a) and 9.5 (b) $l /$ min. The dashed red lines represent the SNR level for FAIMS measurements, under the same conditions, without the lock-in amplifier. Additional experimental parameters are summarized in Appendix D.

Depending on the modulation signal's frequency and amplitude, the lock-in detection technique can have different effects on the FAIMS measurements. The most important effect is the SNR improvement. A well-tuned modulation signal can lead to SNR improvements up to a factor three with very low distortion of the ion spectra's shape and resolution. This reduces the requered measuring time by about one order of magnitude. Figure 4.32 summarizes the modulation frequency effect on the SNR of the lock-in output signals at low (a) and high gas flowrates (b). The dashed red lines represent the SNR level for FAIMS measurements, at the 
same experimental conditions, without the lock-in amplifier. The SNR behavior is very similar for both carrier gas flowrates: at low modulation frequencies the SNR increases with the modulation frequency due to the reduction in the base line noise levels. Once the noise levels stabilize $(\sim 2 \mathrm{~Hz})$, the SNR reaches the maximum value. At about $100 \mathrm{~Hz}$, the SNR starts to decrease again due to the reduction in the ion peak's amplitude. The fluctuations on the SNR are due to the random base line noise fluctuations, not due to signal inconsistencies.Finally, from Figure 4.32.b it can be observed that the SNR versus modulation frequency behavior is the same at different modulation amplitudes. The only difference is the SNR's magnitude which is directly dependent on the signal's amplitude.

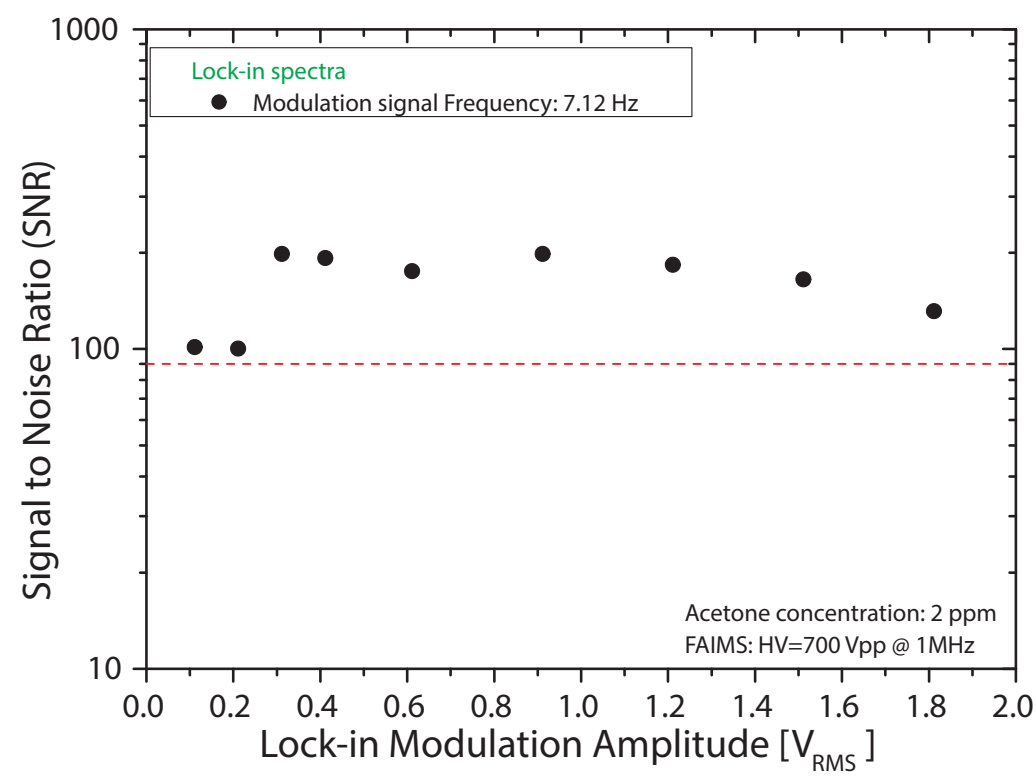

Figure 4.33: Acetone spectra's SNR variation at different modulation amplitudes with a modulation frequency of $7.12 \mathrm{~Hz}$. The dashed orange line represents the SNR level for FAIMS measurements, under the same conditions, without the lock-in amplifier. The carrier gas and the acetone flowrates were kept constant at $3.05 \mathrm{l} / \mathrm{min}$ and $2.5 \times 10^{15}$ molecules/second respectively. The ion filtering was performed at $700 V_{P P}, 1 \mathrm{MHz}$ and $33 \%$ duty cycle. Additional experimental parameters are summarized in Appendix D.

Another factor affecting the SNR is the amplitude of the modulation signal. The modulation amplitude only affects the ion peak's amplitude and FWHM but not the noise levels. Consequently, SNR behavior is governed by the ion peak's amplitude dependency on the modulation amplitude. Figure 4.33 shows the variation of the ion spectra's SNR of the lock-in output signal at different modulation amplitudes.

\subsection{Limit of detection (LoD)}

Once the best experimental conditions were determined, different measurements were performed at low analyte concentration to determine the LoD of the developed FAIMS system. The lowest tested acetone concentration was $8 \mathrm{ppb}$. Producing test gas flows at lower analyte concentrations would require a more complex gas generator setup to ensure the concentration 
stability and to filter all the impurities.

In order to have a high sensitivity level, nitrogen was selected as carrier gas and the relative humidity levels were kept low, i.e. 5\%. Also, the UV lamp input current was increased to $625 \mu \mathrm{A}$. The experimental data from these measurements are plotted in Figures 4.34-4.36. The plots in Figures 4.34 and 4.35 show the detected acetone spectra with the ion filter ON (b) and OFF (a) at low and high gas flowrates. These ion spectra were measured with a sampling frequency of $12.3 \mathrm{kHz}$, a sampling rate of 10 points/volt, an averaging time of 0.1 seconds/ point and only one scan per curve. As can be seen, the ion spectra's shape and CV shift remains constant at the different measured concentrations except for the appearance of a small secondary peak at the at low concentrations and high flowrates. The main effect of the concentration decrease is the reduction in the signal's amplitude. At low concentration, the ions peak's amplitude shows a linear behavior proportional to the injected amount of acetone. However, at high concentrations, the increase in the signal's amplitude starts to deviate from the linear behavior. This can be explained by the light absorption and recombination rates, as described in Section 2.1. The signal's amplitude behavior for the measured acetone concentrations at low (a) and high (b) gas flowrates is summarized in Figure 4.36. The black squares represent the measurements taken with the ion filter OFF and the red circles the measurements taken with an ion filtering of $700 \mathrm{~V}_{\mathrm{PP}}$ at $1 \mathrm{MHz}$ and $33 \%$ duty cycle.

There are different methods to determine the theoretical LoD [25], e.g. graphically, by extrapolating the experimental data, mathematically, by calculating the SNR, etc. In our case, the LoD was resolved by the extrapolation and the SNR based methods. The graphical method is depicted in Figure 4.36: the solid lines show the extrapolation of the experimental data, with (red) and without (black) ion filtering, and the dashed magenta line represents the minimum ion current detection level using the standard detection method defined above. This level is given by three times the standard deviation of the spectra's base line. The LoD is defined by the interception of the solid and dashed lines. Additionally, the LoD was calculated from the detected spectra's SNR:

$$
\begin{aligned}
& L o D=\frac{C}{S N R} \\
& S N R=\frac{A}{3 \sigma}
\end{aligned}
$$

Where $\mathrm{C}$ is the analyte concentration, $\mathrm{A}$ the amplitude of the detected ion peak at an analyte concentration $\mathrm{C}$ and $\sigma$ the standard deviation of the spectra's base line. Both the graphically determined and calculated LoD values for the standard detection method are summarized in Table 4.3. Since the experimental measurements have a very linear behavior, the determined LoD values with both methods are very similar with each other. 

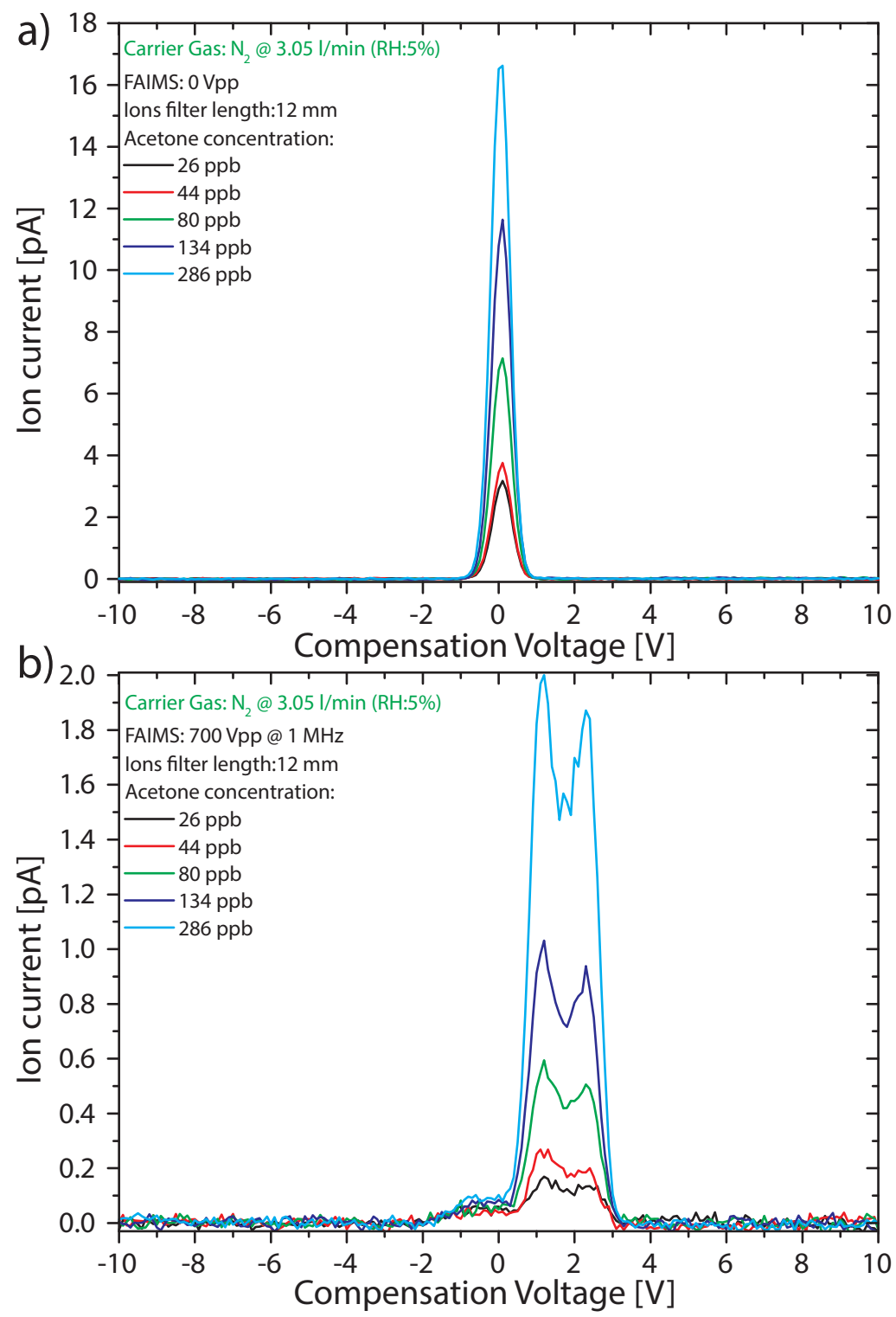

Figure 4.34: Acetone spectra detected at concentrations from 26 to $286 \mathrm{ppb}$ with the ion filter OFF (a) and ON (b). The selected carrier gas was nitrogen with 5\% relative humidity and 3.05 l/min flowrate. The ion filtering in (b) was performed at $700_{P P}, 1 \mathrm{MHz}$ and $33 \%$ duty cycle. The ion current was measured with a sampling frequency of $12.3 \mathrm{kHz}$, a sampling rate of 10 points/volt and an averaging time of 0.1 seconds/ point, i.e. $\sim 20$ seconds per scan. 

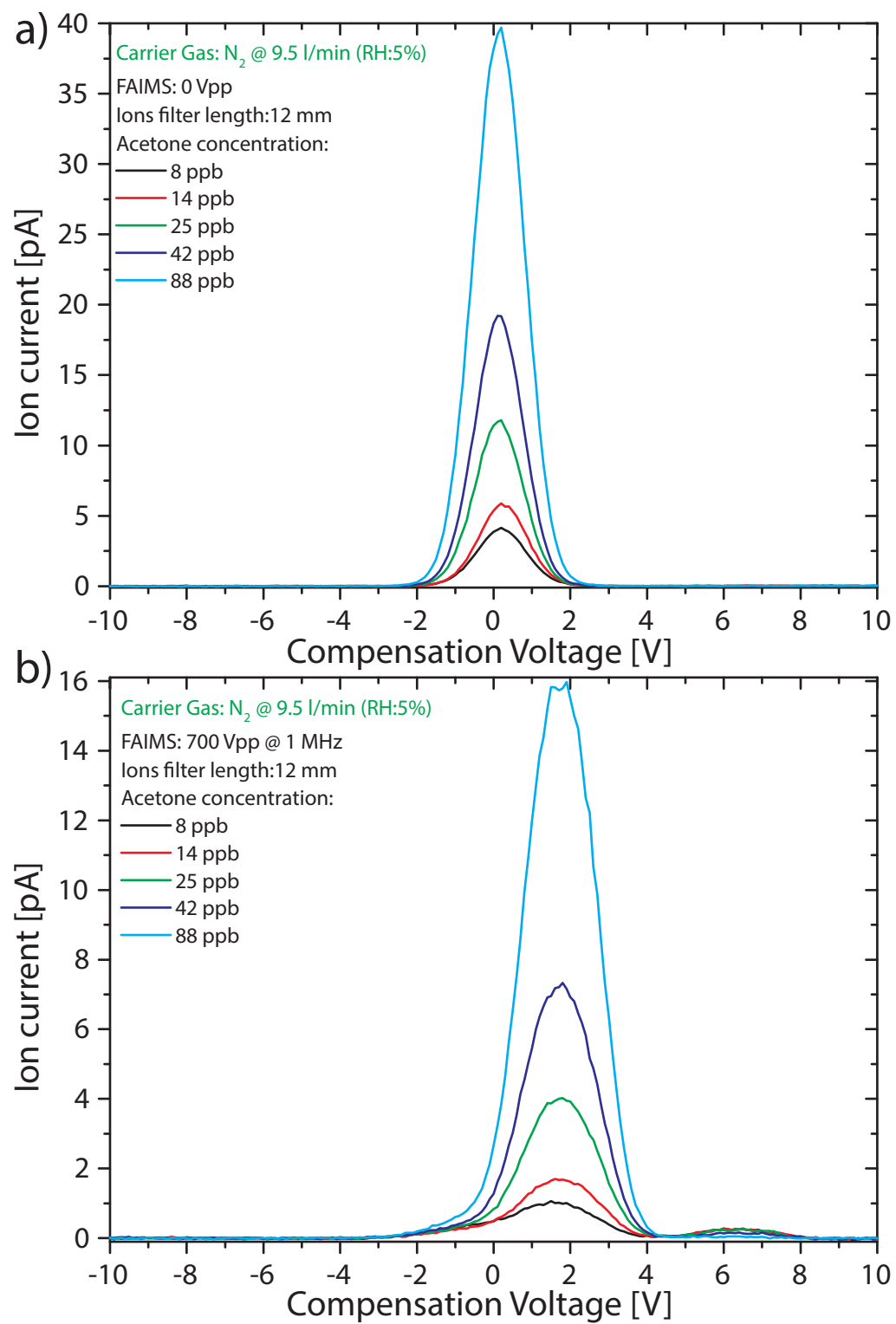

Figure 4.35: Acetone spectra detected at concentrations from 8 to $88 \mathrm{ppb}$ with the ion filter OFF (a) and $\mathrm{ON}(\mathrm{b})$. The selected carrier gas was nitrogen with $5 \%$ relative humidity and $9.5 \mathrm{l} / \mathrm{min}$ flowrate. The ion filtering in (b) was performed at $700 V_{P P}, 1 \mathrm{MHz}$ and 33\% duty cycle. The ion current was measured with a sampling frequency of $12.3 \mathrm{kHz}$, a sampling rate of 10 points/volt and an averaging time of 0.1 seconds/ point, i.e. $\sim 20$ seconds per scan.

Since the noise level of the spectra's baseline directly affects the FAIMS LoD, it can be improved by tuning the measurement parameters. Figure 4.36 show the variations in the minimum current detection levels at different measurement conditions. First, the measurements' bandwidth was reduced by increasing the averaging time per point from 0.1 to $1 \mathrm{~s}$. This results in an increase of the SNR levels between 1.5 and 2.5 times and, therefore, a reduction in the LoD of the same magnitudes. Another tested measurement approach was to keep the averaging time at $0.1 \mathrm{~s}$ but increase the amount of scans to 10 . This improved the signal's SNR and LoD 
about 3 times. Even though in both cases the measurement's bandwidth was reduced by the same amount, taking several fast scans leads to a better $1 / \mathrm{f}$ noise filtering than performing one single slow scan, which explains the higher SNR improvement for the second approach.
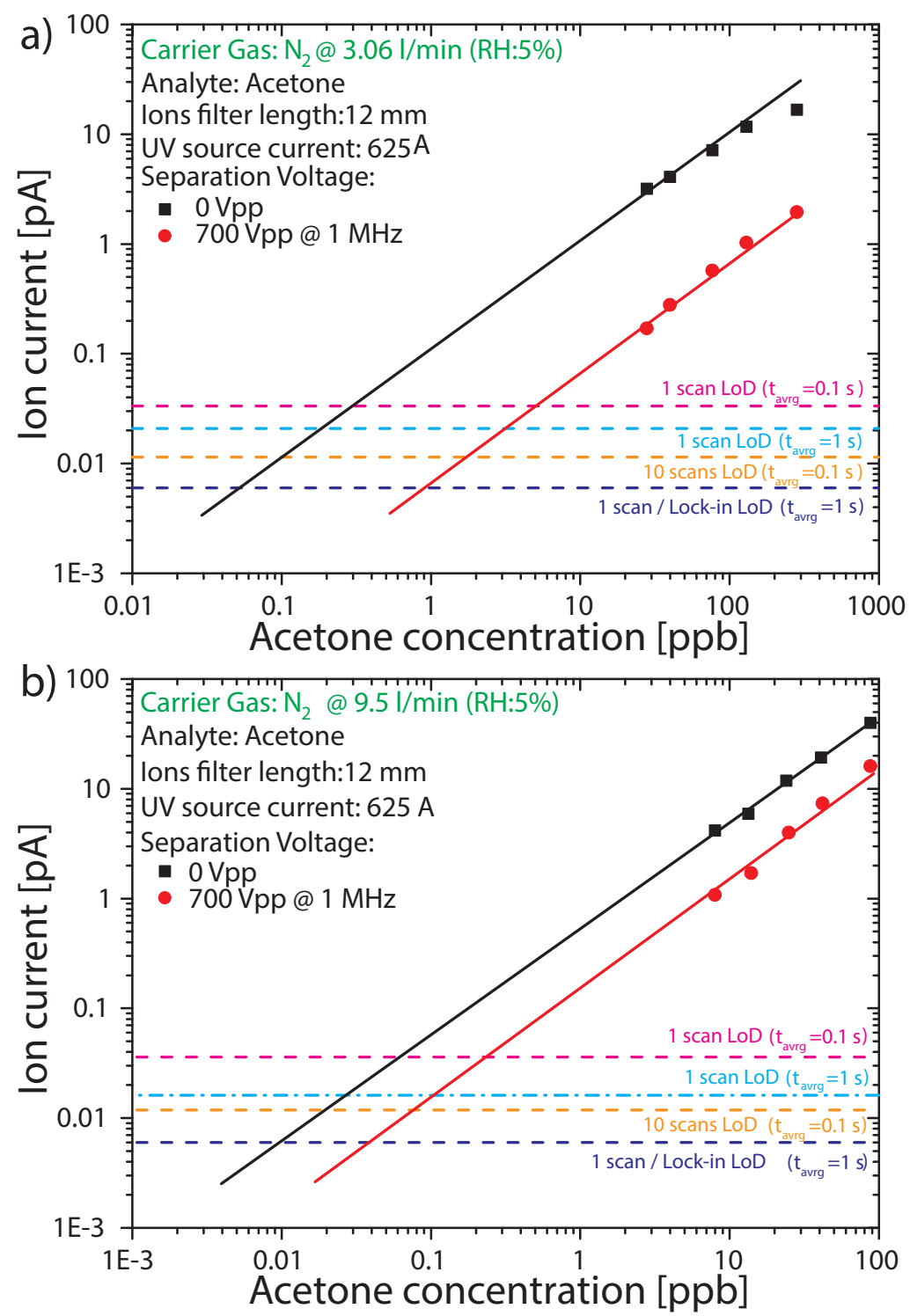

Figure 4.36: Variation of the ions peak amplitude at different acetone concentration levels. The selected carrier gas was nitrogen with 5\% relative humidity and flowrates of 3.05 (a) and 9.5 (b) $l /$ min. The measurements in black were performed with the ion filter OFF and the red ones with the filter $\mathrm{ON}$ at $700 \mathrm{~V}_{P P}, 1 \mathrm{MHz}$ and 33\% duty cycle. The red and black symbols show the experimental data, the red and black lines represent the linear fitting of the experimental measurements and the dashed lines the minimum ion current detection level with different measurement parameters. 


\begin{tabular}{|c|c|c|c|}
\hline $\begin{array}{c}\text { Carrier gas } \\
\text { flowrate }\end{array}$ & $\begin{array}{c}\text { Separation } \\
\text { Voltage }\end{array}$ & $\begin{array}{c}\text { Graphically } \\
\text { determined LoD }\end{array}$ & $\begin{array}{c}\text { Calculated } \\
\text { LoD }\end{array}$ \\
\hline $3.06 \mathrm{l} / \mathrm{min}$ & $0 \mathrm{~V}_{\mathrm{PP}}$ & $296 \mathrm{ppt}$ & $290 \mathrm{ppt}$ \\
\cline { 2 - 4 } & $700 \mathrm{~V}_{\mathrm{PP}}$ & $3.34 \mathrm{ppb}$ & $3.38 \mathrm{ppb}$ \\
\hline \multirow{2}{*}{$9.5 \mathrm{l} / \mathrm{min}$} & $0 \mathrm{~V}_{\mathrm{PP}}$ & $64 \mathrm{ppt}$ & $67 \mathrm{ppt}$ \\
\cline { 2 - 4 } & $700 \mathrm{~V}_{\mathrm{PP}}$ & $240 \mathrm{ppt}$ & $236 \mathrm{ppt}$ \\
\hline
\end{tabular}

Table 4.3: Comparizon between the graphically determined and the calculated LoD levels at different carrier gas flowrates, with and without ion filtering. Measurement conditions: sampling frequency of $12.3 \mathrm{kHz}$, sampling rate of 10 points/volt, averaging time of 0.1 seconds/ point and one scan per measurement.

The last analyzed detection method was the lock-in modulation/demodulation process. Using this method allowed one to reduce the measurements' bandwidth and shift it to the low noise section of the noise spectra, leading to SNR improvements of up to 3 times, in comparison to the measurements without Lock-in at the same conditions, i.e. 1 scan, $1 \mathrm{~s}$ averaging time. The calculated FAIMS LoD using the Lock-in detection method is presented in Table 4.4.

\begin{tabular}{|c|c|c|}
\hline \multirow{2}{*}{$\begin{array}{l}\text { Carrier gas } \\
\text { flowrate }\end{array}$} & \multicolumn{2}{|c|}{ Limit of detection (LoD)for acetone } \\
\hline & HV: 0 Vpp & HV: 700 Vpp @ 1 MHz \\
\hline \multirow{4}{*}{$\begin{array}{l}3.061 / \mathrm{min} \\
\left(1.25 \times 10^{21}\right. \\
\text { molecules/s) }\end{array}$} & $50 \mathrm{ppt}$ & $900 \mathrm{ppt}$ \\
\hline & $6.25 \times 10^{10}$ molecules $/ \mathrm{s}$ & $1.1 \times 10^{12}$ molecules $/ \mathrm{s}$ \\
\hline & $7.7 \mu \mathrm{m}^{3} / \mathrm{s}$ & $140 \mu \mathrm{m}^{3} / \mathrm{s}$ \\
\hline & $2 \times 2 \times 2 \mu \mathrm{m}^{3} / \mathrm{s}$ & $5.1 \times 5.1 \times 5.1 \mu \mathrm{m}^{3} / \mathrm{s}$ \\
\hline \multirow{4}{*}{$\begin{array}{l}9.51 / \mathrm{min} \\
\left(3.95 \times 10^{21}\right. \\
\text { molecules/s) }\end{array}$} & $10 \mathrm{ppt}$ & $40 \mathrm{ppt}$ \\
\hline & $3.9 \times 10^{10}$ molecules $/ \mathrm{s}$ & $1.58 \times 10^{11}$ molecules $/ \mathrm{s}$ \\
\hline & $4.9 \mu \mathrm{m}^{3} / \mathrm{s}$ & $19 \mu \mathrm{m}^{3} / \mathrm{s}$ \\
\hline & $1.7 \mathrm{x} 1.7 \mathrm{x} 1.7 \mu \mathrm{m}^{3} / \mathrm{s}$ & $2.7 \times 2.7 \times 2.7 \mu \mathrm{m}^{3} / \mathrm{s}$ \\
\hline
\end{tabular}

Table 4.4: Calculated LoD of the developed FAIMS system using the lock-in amplifier setup. The LoD was calculated based on experimental results at low and high gas flowrates with and without ion filtering. The data is converted to different analyte flow units to facilitate the comparison with other publications and techniques.

\subsection{Conclusions}

The experimental parameters influencing the FAIMS sensitivity and resolution were analyzed. These parameters can have different effects on each of the processes taking place during the FAIMS measurements. Therefore, each of the FAIMS subsections, i.e. ionization region, ion filter and detector, were studied separately.

In the ionization region, the main factors affecting the system's sensitivity are the ionization efficiency and the ion losses. The ionization efficiency can be improved by increasing the UV lamp's input current and using a carrier gas with a low light absorption coefficient. The 
amount of ion losses can be decreased by reducing the ions' traveling time between the ionization region and the detector. This can be accomplished by shrinking the ion filter region or by increasing the carrier gas flowrate. However, reducing the ions' residence time in the filter region also leads to a widening of the ion peaks of about half the gain in the amplitude. Another option to reduce the ions recombination is to decrease the gas pressure inside the ions' channel.

Depending on the experimental conditions, the ratio between the amount of detected ions and the input flow of analyte molecules is between $1 \times 10^{-7}$ and $1 \times 10^{-9}$. The ion losses experiments let to ratios between the produced and the detected ions between 0.5 and 0.01 . Therefore, the ionization efficiency of the UV source is around $1 \times 10^{-6}$ and $1 \times 10^{-7}$. This is similar to the ionization efficiency values for PID systems reported in literature [1].

In the ion filter region, the coupled noise from the HV signal was reduced using a second order LP filter and shielding the FAIMS core, ion filter circuits and low current amplifier in different metallic boxes. This improved the SNR measurements by almost two orders of magnitude. However, the LP filter slows down the HV circuit deforming the separation voltage signal, which reduces the CV shift about $15 \%$.

The first section of this chapter showed that reducing the ions traveling time between the ionization region and the detector improves the FAIMS sensitivity. However, it also reduces the ion filtering effect and, therefore, the system's resolution. From the tested methods aiming to reduce the ions' traveling time, increasing the gas flowrate was the one that least reduced the resolution: increasing the flowrate four times led to an improvement in the systems sensitivity of about 50 times with a resolution loss of only $30 \%$. Similarly, decreasing the amount of humidity in the carrier gas reduces the light absorption, which improves the ionization efficiency and the FAIMS sensitivity. However, it also reduces the CV shift and the systems resolution, e.g. decreasing the humidity levels from $40 \%$ to $5 \%$ increased the signal amplitude about $55 \%$ but reduced the CV by approximately $70 \%$.

Another tested parameter was the gas pressure inside the ion channel. Decreasing the pressure reduces the ions recombination and increases the mobility. Therefore, when no electric field is applied in the channel, working at low pressures improves the sensitivity. However, once the HV signal is applied, the higher mobilities increase the amplitude of the ions' oscillation inside the channel reducing the effective ions' gap and the system's sensitivity. A positive effect of the pressure reduction in the ion filtering is the increase in the CV shift and, therefore, the system's resolution. Likewise, decreasing the filtering frequency increase the amplitude of the ions' oscillation inside the channel reducing the system's sensitivity. Nevertheless, since the ion mobilities are not altered, the $\mathrm{CV}$ shift remains constant regardless of the frequency variations. Finally, the resolution of the developed FAIMS was evaluated by measuring two different analytes, i.e. acetone and o-xylene, at different separation voltages. The detected spectra allowed the clear identification of both compounds at separation voltages higher than $350 \mathrm{~V}_{\mathrm{PP}}$. From the analysis of the ion detector, an optimum deflection voltage value of about $5 \mathrm{~V}$ was identified.

For the first time, the lock-in modulation/demodulation technique was implemented in the FAIMS measurements. Adding a lock-in amplifier stage to the FAIMS system led to signal 
to noise ratios up to 3 times higher than direct FAIMS measurements. This technique can be very useful for FAIMS systems since it can improve the limit of detection (LoD) by the same magnitude (3x) and reduce the required measurement time by about one order of magnitude without significant modifications in the measurements setup. Nevertheless, the modulation parameters (frequency and amplitude) must be carefully tuned to avoid reducing the system's resolution or SNR degradation. The main tunable parameters in lock-in detection are the averaging time and the modulation signal amplitude and frequency. The performed experiments showed that increasing the averaging time reduces the measurement bandwidth and, therefore, the signal noise. However, it also slows down the response to the signal changes. Also, increasing the modulation amplitude increases the ion peak's amplitude, until the signal starts to be over modulated. At this point, the ion peak's amplitude saturates and the FWHM starts to increase. Finally, increasing the modulation frequency rapidly decreases the detected noise levels, which increases the SNR. However, the noise reduction stops at about $2 \mathrm{~Hz}$ and the SNR reaches the maximum. At about $100 \mathrm{~Hz}$, the ions peak amplitude start to decrease reducing the SNR.

In the final section of this chapter, two sets of experiments, at low and high gas flowrates, were preformed to identify the LoD of the developed FAIMS device. The selected analyte and carrier gas were acetone and nitrogen, respectively. The measurements were done under the most suitable experimental conditions identified from the previous analysis. Also, the detection parameters, such as sampling frequency, rate and averaging time, were tuned to determine the best measuring conditions. The best results were obtained with the lock-in modulation/demodulation technique: at a low flowrates $(3.06 \mathrm{l} / \mathrm{min})$, the system's LoD is 50 ppt $\left(6.25 \times 10^{10}\right.$ molecules/s) without ion filtering and $900 \mathrm{ppt}\left(1.1 \times 10^{12}\right.$ molecules/s) with an ion filtering of $700 \mathrm{~V}_{\mathrm{PP}}$ at $1 \mathrm{MHz}$. At high flowrates $(9.5 \mathrm{l} / \mathrm{min})$, the system's LoD improves to $10 \mathrm{ppt}\left(3.9 \times 10^{10}\right.$ molecules/s) without ion filtering and $40 \mathrm{ppt}\left(1.58 \times 10^{11}\right.$ molecules/s) with an ion filtering of $700 \mathrm{~V}_{\mathrm{PP}}$ at $1 \mathrm{MHz}$. These acetone LoD levels are at the same level or slightly better than the state of the art ( 1.5 ppb) [31, 32, 33]. 



\section{Conclusions and Outlook}

\subsection{Conclusions}

The main objective of this work was to improve the limit of detection in FAIMS. With this aim, the parameters affecting the system's sensitivity were thoroughly analyzed by theoretical and experimental methods.

For the theoretical study, an ion mobility simulation software was developed based on the physical models proposed in literature. With this software, it is possible to simulate the effect of different parameters on the ions' trajectories inside the filter and the final ion spectra. The simulated results match the experimental results obtained in our lab and those of other groups published in different papers.

In order to validate the simulated results with experimental data, four FAIMS systems were designed and fabricated. The FAIMS electrode plates were prototyped using two different sets of materials (alumina + silver-palladium and FR 4 + copper) and fabrication techniques (screen printing, PCB prototyping). The performance of both the alumina and PCB systems was very similar. Therefore, producing the FAIMS cores with the PCB fabrication methods is a good option to reduce the fabrication costs, times and complexity without affecting the measurements' quality.

The ionization of the analyte molecules was performed by UV photoionization at atmospheric pressure. The selected UV lamp emits photons with wavelengths of 124 and $117 \mathrm{~nm}$, which can ionize molecules with potentials of $10.6 \mathrm{eV}$ or lower. This ionization threshold has a positive effect in the system's selectivity, since only the analyte (VOC) molecules are ionized but not the carrier gas components. A corona discharge ionization system was also implemented with an efficiency level similar to the UV ionization. However, it must be carefully tuned to avoid coupling high amounts of electrical noise into the measurement signal. Also, the corona's tip electrode rapidly loses its sharpness, which led to variations in the ionization behavior. Therefore, it was decided to discard this ionization system and perform all the experiments with the UV source.

Two electronic circuits were developed to generate the separation and compensation voltage signals required by the ion filter. The HV circuit is based on a power n-MOS inverter configuration and it is capable to produce square waveforms with peak to peak voltages up to 1000 $\mathrm{V}_{\mathrm{PP}}$ at frequencies in the $\mathrm{kHz}$ and $\mathrm{MHz}$ ranges and different duty cycles. The $\mathrm{CV}$ circuit is controlled by the user's interface software and can generate voltage ramps from - 20 to $20 \mathrm{~V}$. 
Finally, the circuits were shielded in a metallic box and a second order LP filter was added to filter the HV signal. This reduces the coupled noise levels and improved the measurements' SNR by almost two orders of magnitude.

The detected ion currents are amplified by a low noise current amplifier and digitalized by a DAQ board. The measured signals are then displayed by the user's interface and saved in a data file for further analysis. The developed interface software also allows the user to control the acquisition frequency, rate, averaging time, deflection voltage, among other parameters. The designed low noise current amplifier has a total gain of $1 \times 10^{11}$ with very low noise level of about $4 \mathrm{fA} / \sqrt{\mathrm{Hz}}$, limited by the thermal noise of the feedback resistor.

The experimental setup also includes a calibration gas generator, a gas pump and different sensors to control and monitor the experimental conditions. The carrier gas flow can be generated by a pressurized gas bottle or the gas pump. However, using the pump slightly increases the noise levels in the measured signal. The calibration gas generator, fabricated by Owlstone Ltd., controls the gas flowrate and desorbs the analyte. It can produce stable carrier gas flows from $\mathrm{ml} / \mathrm{min}$ to $1 / \mathrm{min}$ with analyte concentrations between ppt and ppm levels. The gas bottles, the mass flow controllers in the gas generator and the gas pump can be combined to perform low pressure measurements.

In the experimental characterization, several parameters were tuned to determine their effect on the FAIMS sensitivity and resolution. Except for the UV lamp's input current and the ion filter frequency, most of the tested parameters required a compromise between sensitivity and resolution. Increasing the UV lamp's current or the ion filtering frequency improves the system's sensitivity with a very small or no effect on the resolution. Increasing the carrier gas flowrate or the ions' gap also improves the sensitivity but it reduces the resolution as well. Conversely, increasing the separation voltage or the ion filter's length improves the FAIMS resolution but reduces sensitivity. Finally, selecting a carrier gas with a low light absorption coefficient and reducing the contamination levels improves the system's sensitivity. However, depending of the type of gas or contaminant, the effects on the resolution will change, e.g. decreasing the humidity levels improves the sensitivity but reduces the resolution.

From the experimental characterization was also concluded that the ionization efficiency $\left(1 \times 10^{-6}-1 \times 10^{-7}\right)$ has a much larger effect on the rate between the amount of detected ions and the input analyte molecules than the ion losses (0.5-0.01). Therefore, the best approach to improve the FAIMS is to implement a more efficient ionization source, instead of reducing the ion losses by increasing the gas flowrates or shrinking the filter region. Furthermore, the mentioned methods to reduce the ion losses also reduce the system's resolution, while improving the ionization efficiency does not.

In order to evaluate the ion filtering capability of the developed FAIMS system, several measurements of acetone and o-xylene were taken at separation voltages from 0 to $750 \mathrm{~V}_{\mathrm{PP}}$. The resulted spectra allowed one to clearly distinguish between both compounds at separation voltages higher than $350 \mathrm{~V}_{\mathrm{PP}}$. Also, the detected spectra agree with results found in literature [7].

Since the FAIMS LoD not only depends on amount of ions but also on the spectra's base line noise, different measuring techniques were investigated to improve the detected SNR. The best 
results were obtained with the lock-in modulation/demodulation technique. Despite being use in different fields for many years, this is the first time, to the best of our knowledge, that the lock-in detection technique has been used in FAIMS measurements. A lock-in amplifier uses a modulation signal and a LP filter to narrow the measurement bandwidth and shift it to a low noise section of the spectra. This way, it is possible to extract small signals from very noisy environments. The results of implementing this technique were very promising: by properly tuning the modulation amplitude and frequency, the SNR levels were improved up to three times with a very small reduction of the system's resolution.

Once the most appropriate experimental conditions and detection techniques were defined, a set of measurements was performed to determine the LoD of the developed FAIMS system. Taking the measurements at low humidity levels, high filtering voltages and frequencies, high UV input currents, room pressure and using the lock-in detection method, led to LoD levels down to 900 and 40 ppt of acetone at low and high nitrogen flowrates, respectively. This corresponds to an analyte flow of about $1.1 \times 10^{12}$ and $1.6 \times 10^{11}$ acetone molecules per second, i.e. desorbing an acetone cube with lateral length of 5.1 and $2.7 \mu \mathrm{m}$ per second, respectively. The obtained LoDs are at about the same level or slightly better than the acetone LoDs for standalone FAIMS reported in literature [31,32,33]. However, since the published papers do not always contain all the relevant measurement parameters, e.g. measuring time, equivalent noise band width, it is not possible to make a precise comparison. These sensitivity improvements can expand the application fields for FAIMS technology to, for example, chemical imaging with $\mu \mathrm{m}$ resolution. Also, it can improve existing or under development techniques, such as breath analysis for cancer detection, where the analyte concentrations are in the ppt and low ppb levels [33].

\subsection{Outlook}

The presented work contributes to the understanding of the factors affecting the FAIMS resolution and sensitivity and is a first step to the development of high sensitivity applications using FAIMS. Therefore, on both theoretical and experimental fields, there are ample opportunities for future work:

- The developed ion mobility simulation software is a very useful tool to understand and anticipate the experimental results. The simulated data has shown good agreement with the real measurements from different sources. However, there are some parameters that were not or only marginally included in the software's physics model: ion recombination, natural diffusion, initial ion distribution. Therefore, including these parameters in the ion mobility model will lead a more accurate description to the real behavior of the ions and a closer approximation to the measured ion spectra.

- Further improvements in the system's sensitivity, without affecting the resolution, can be achieved by implementing faster power switching devices, to increase the filtering frequency, and more powerful UV sources. Also, adding gas filters and humidity regulators will give the user a better control on the measurements' sensitivity and resolution. 
- Once the FAIMS device has been characterized, performing measurements in new research fields might lead to very interesting results. The most promising fields are breath analysis and chemical imaging. Some studies in cancer detection by breath analysis using FAIMS have been already published [33]. The concentration of the detected cancer markers are in the ppt and low ppb levels, which are much higher than the LoD of the developed system. The imaging of chemical compounds with resolution in the $\mu \mathrm{m}$ range have been achieved using mass spectrometer systems $[18,19,20]$ but have not been done with a FAIMS device. A good starting point to replicate these results using the developed FAIMS device, would be to implement a cantilever based desorption system, such as the NanoFrazor tool produced by SwissLitho AG. The NanoFrazor system is capable to desorb different samples with $\mathrm{nm}$ and $\mu \mathrm{m}$ resolution at rates up to several tens of $\mu \mathrm{m}^{3}$ per second, which is within the detection capabilities of our FAIMS device. 
A Flowchart of the ion mobility simulation software 
Appendix A. Flowchart of the ion mobility simulation software

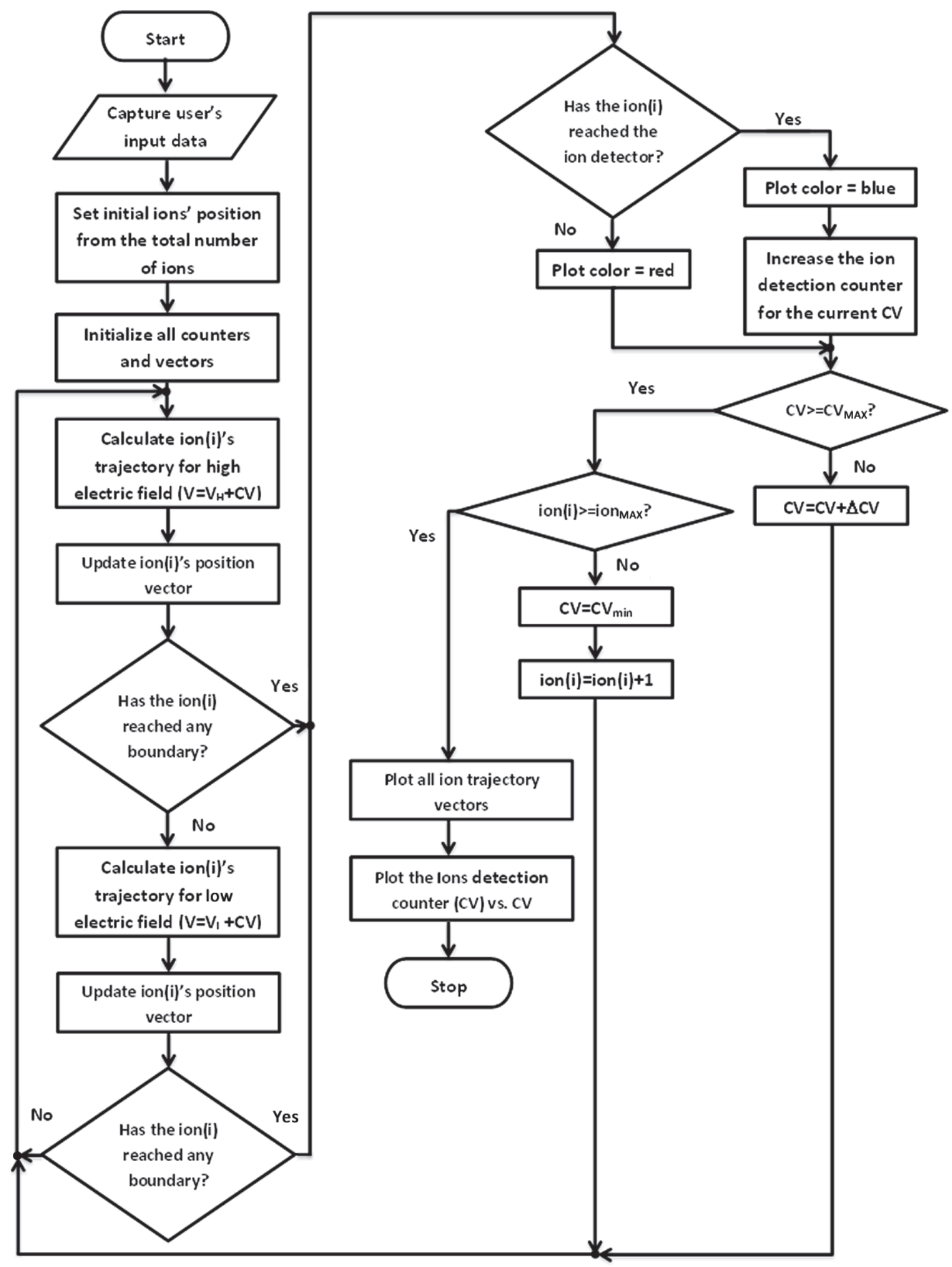


B FAIMS electrode plates designs 

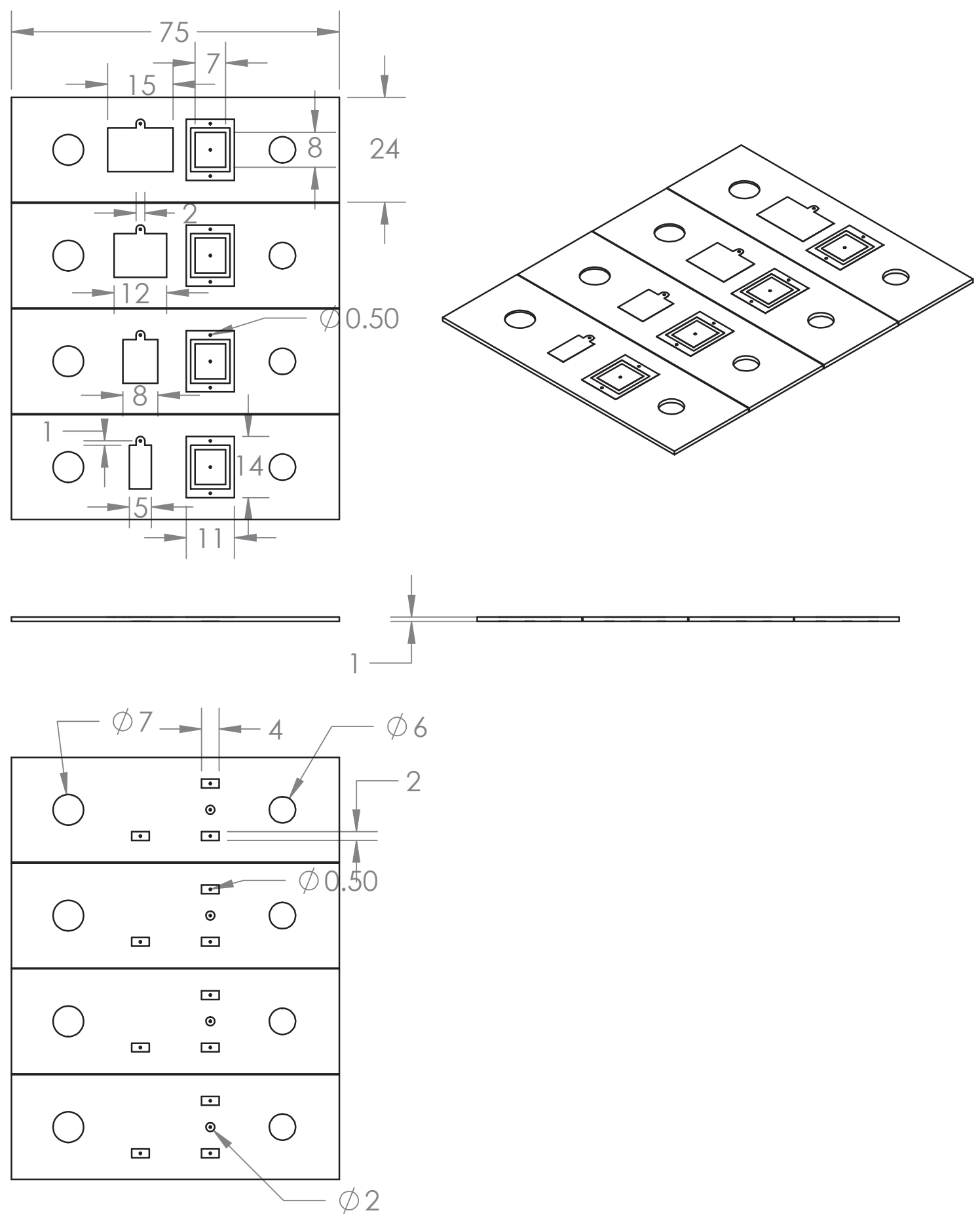

Figure B.1: Drawings of the upper alumina electrodes plates with filter electrodes from 8 to 15 mm long (Dimensions in $\mathrm{mm}$ ). 

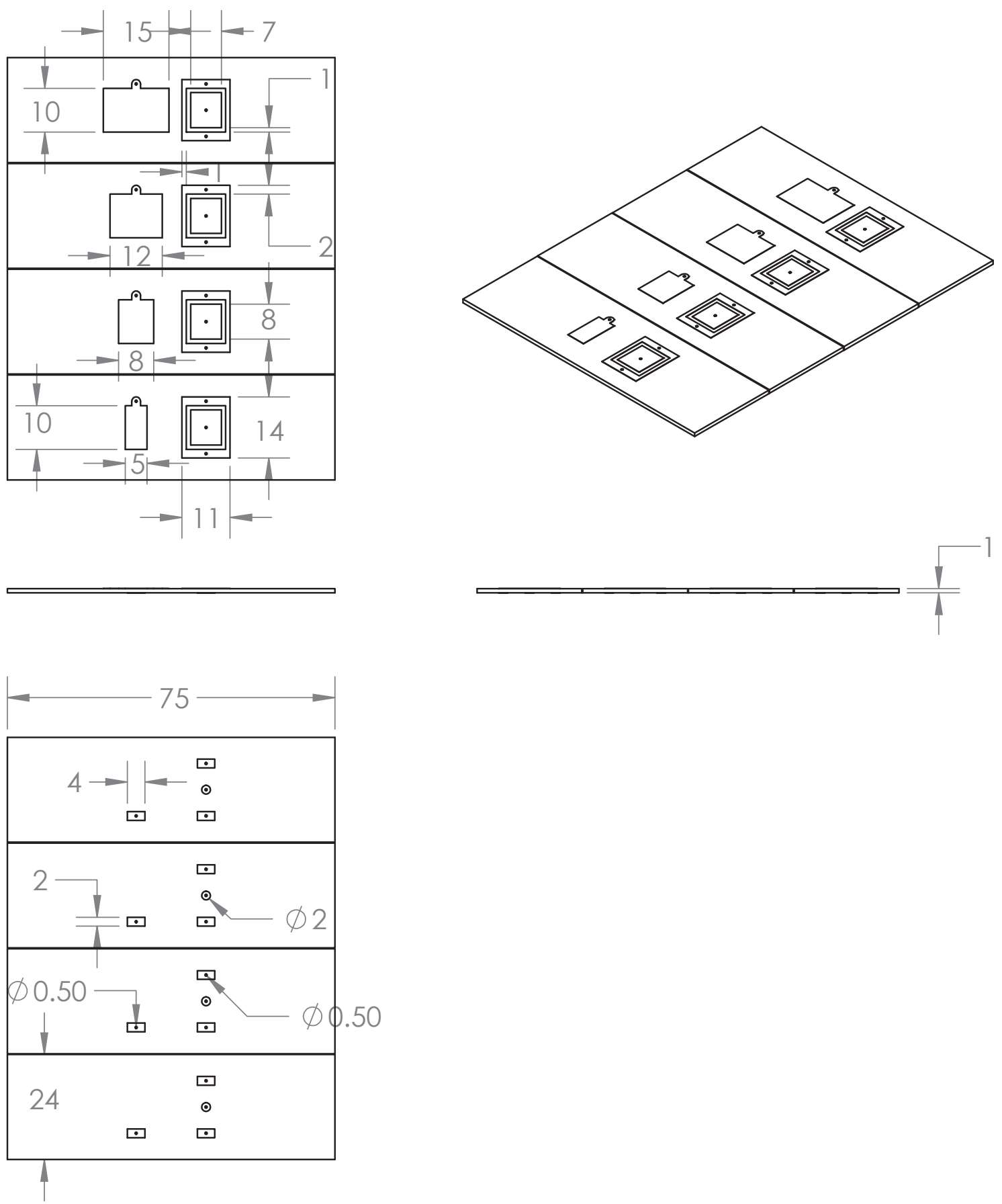

Figure B.2: Drawings of the bottom alumina electrodes plates with filter electrodes from 8 to 15 mm long (Dimensions in $\mathrm{mm}$ ). 


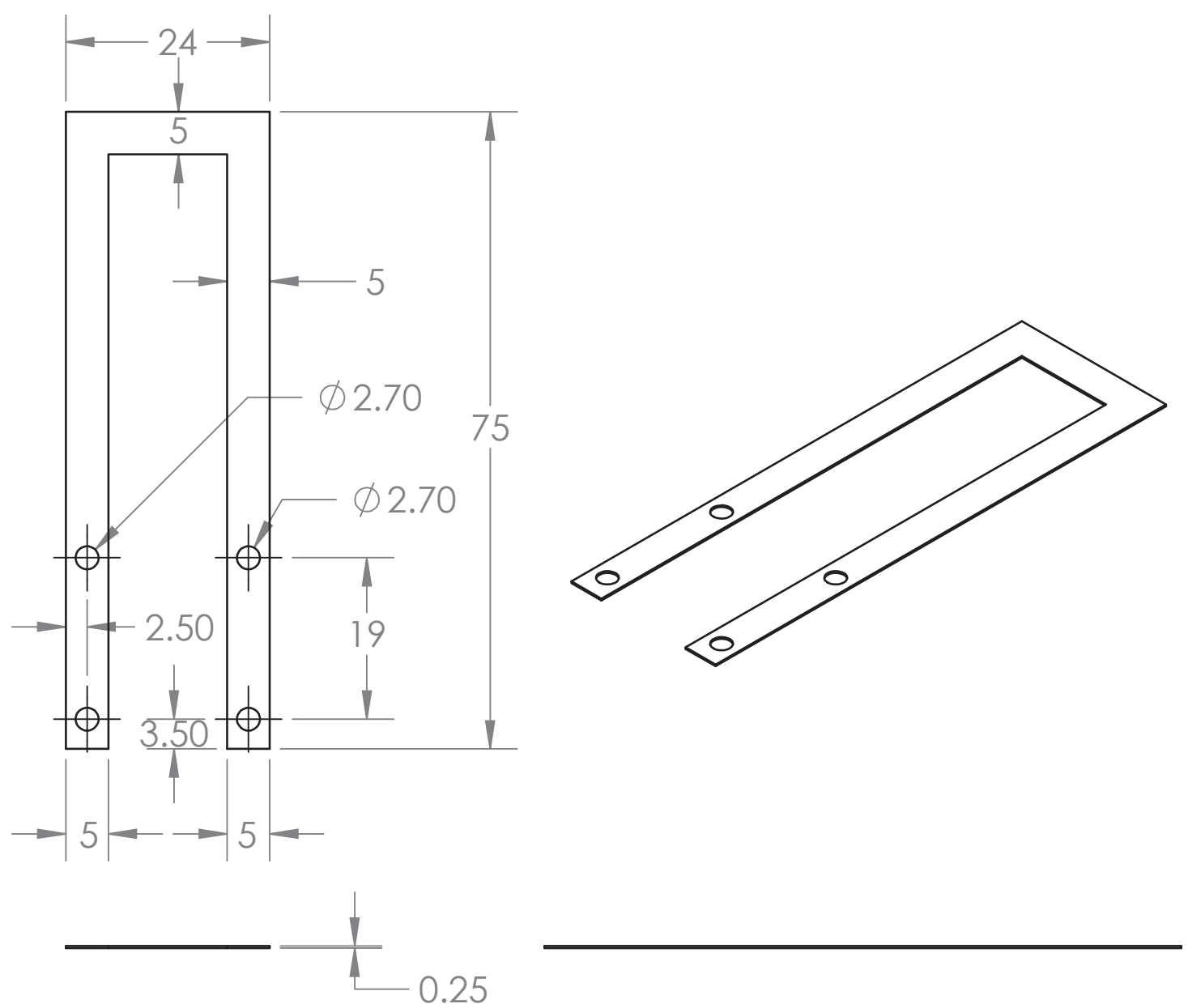

Figure B.3: Drawings of the alumina spacer. The standard implemented thickness was $250 \mu \mathrm{m}$. However, spacers with thicknesses of 170 and $500 \mu \mathrm{m}$ were also fabricated (Dimensions in $\mathrm{mm}$ ). 

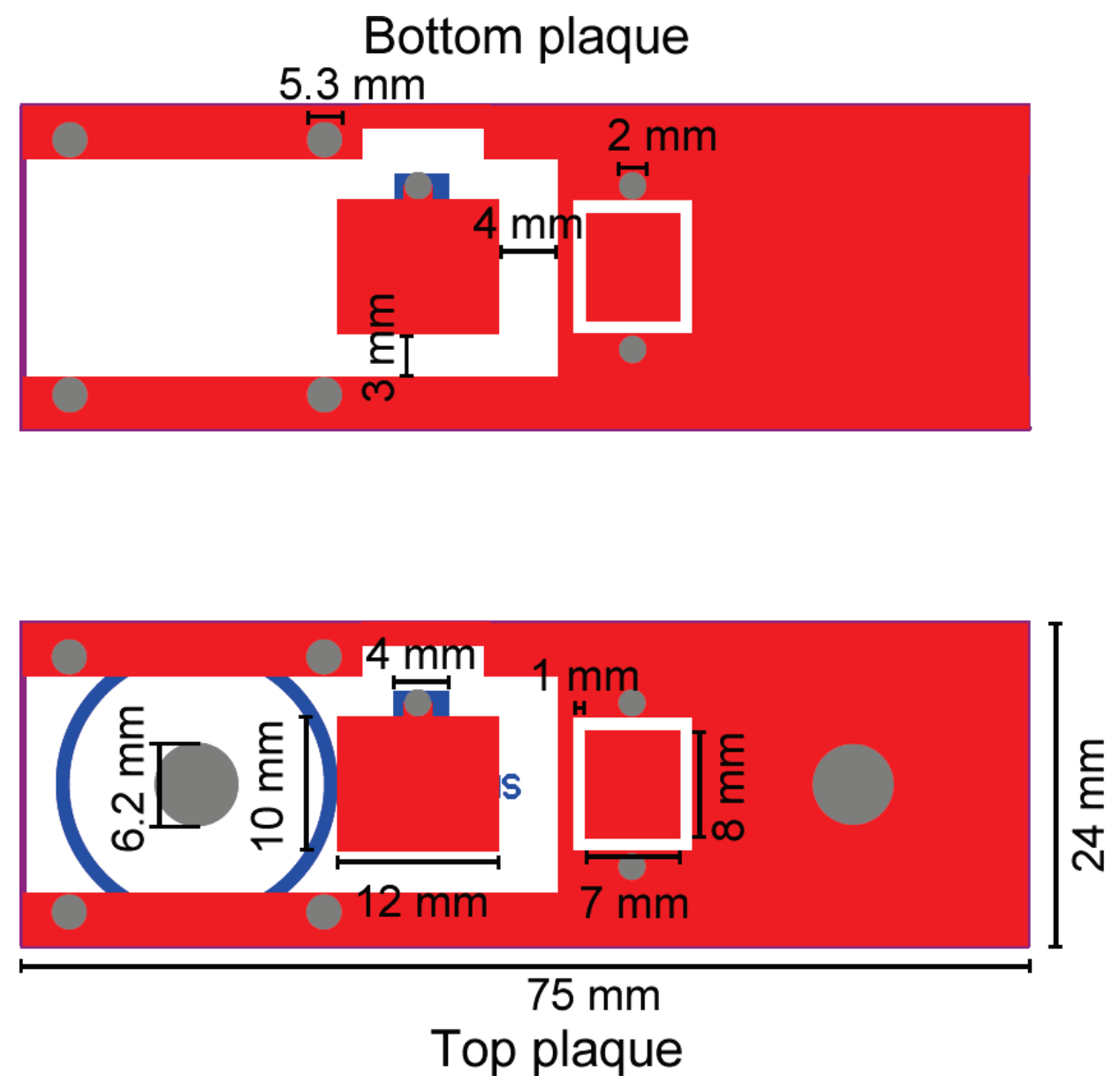

Figure B.4: Drawings of the top and bottom PCB electrodes plates. 


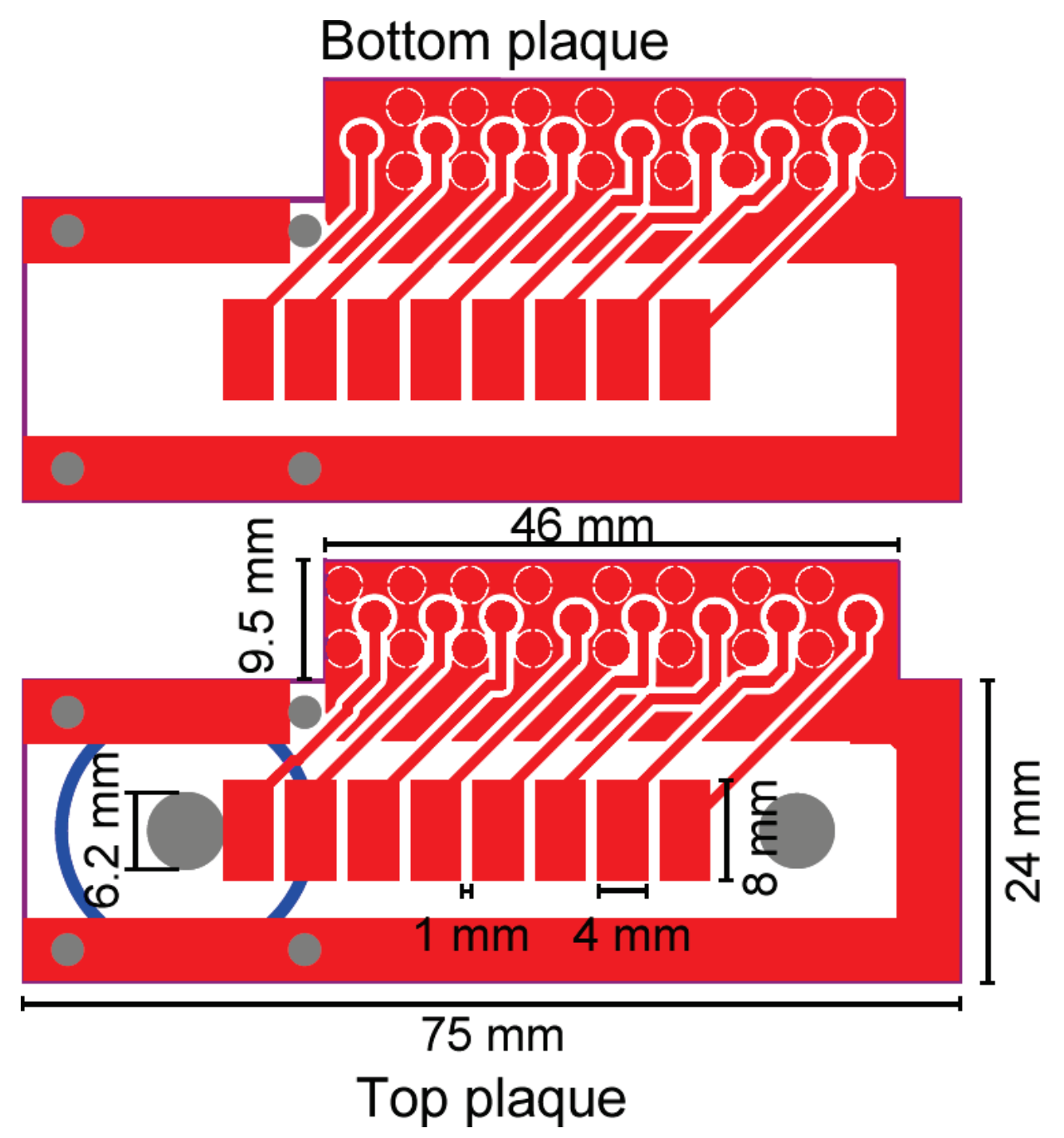

Figure B.5: Drawings of the top and bottom PCB electrodes plates for ion losses measurement. 
C LabVIEW block diagrams of the developed signal processing and user interface software 
Appendix C. LabVIEW block diagrams of the developed signal processing and user interface software

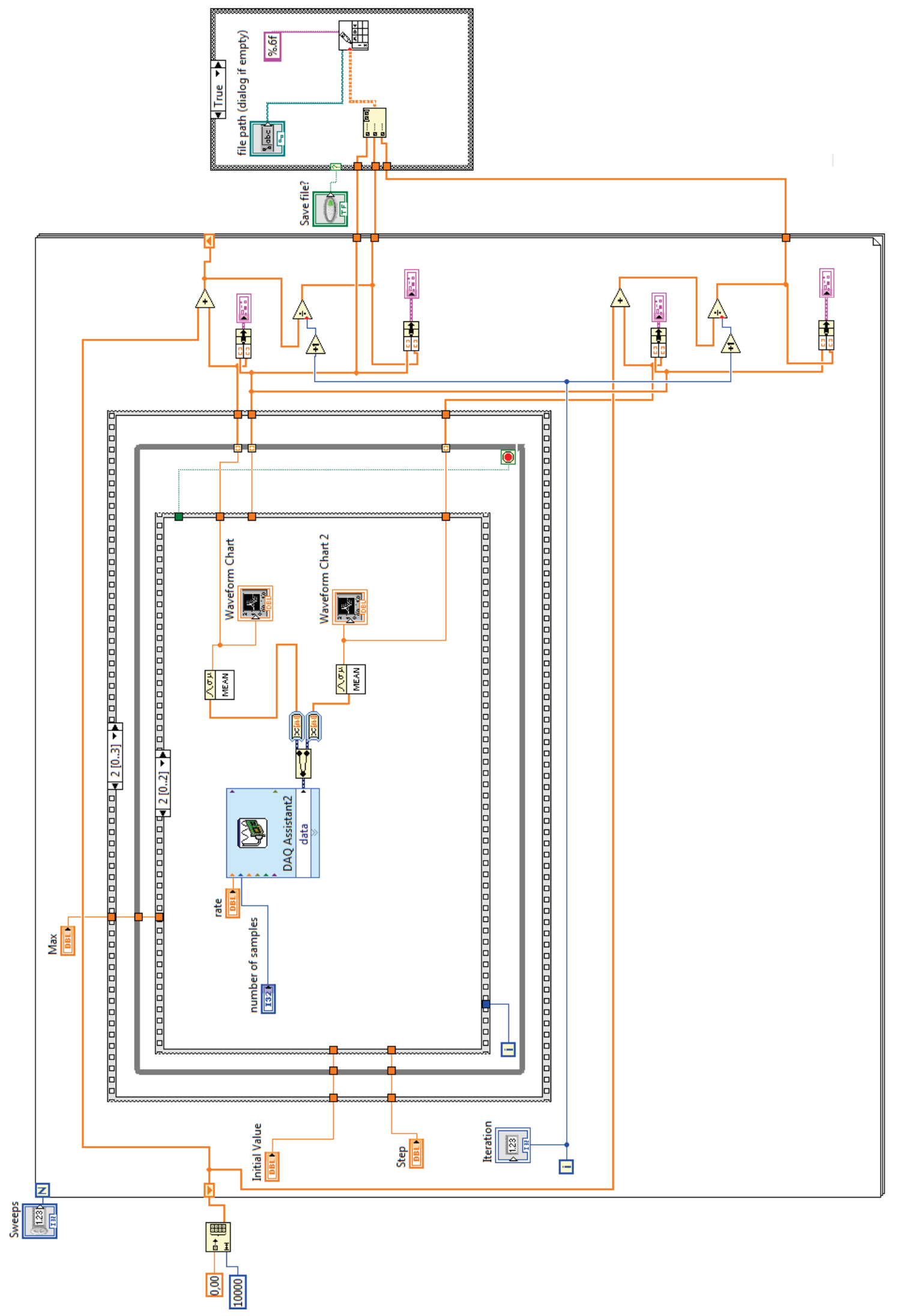




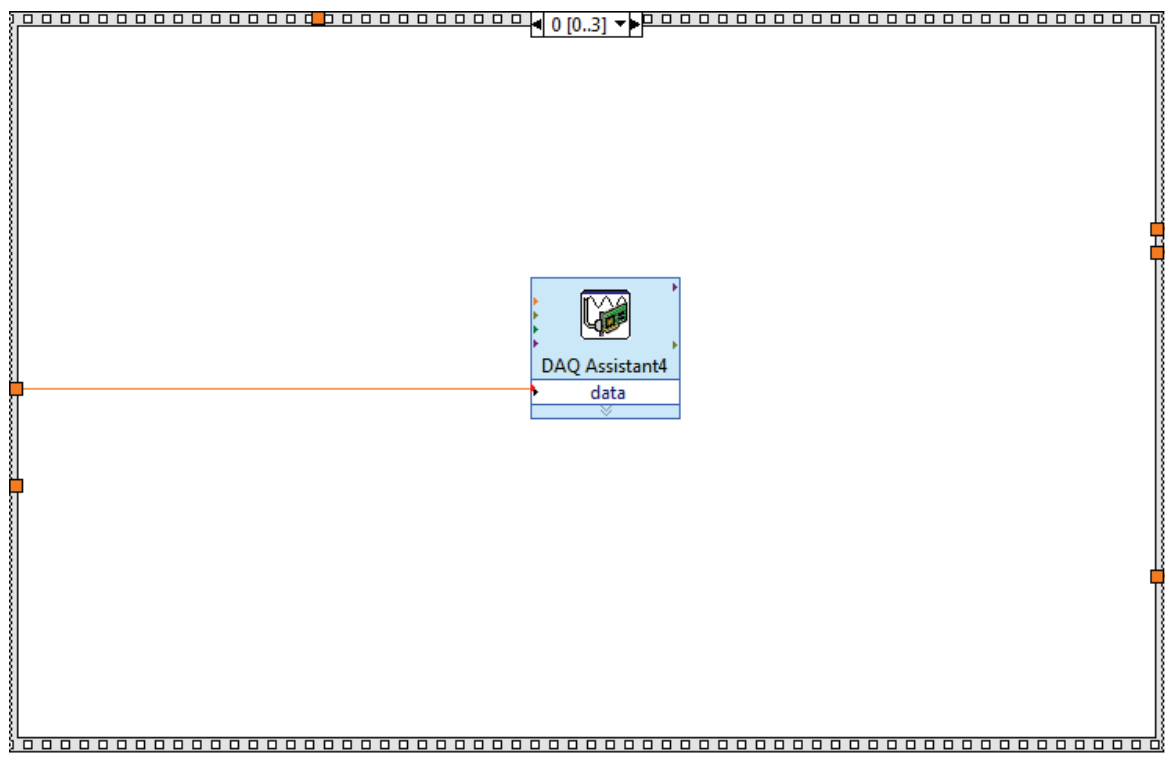

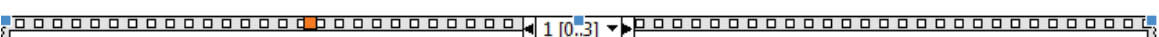

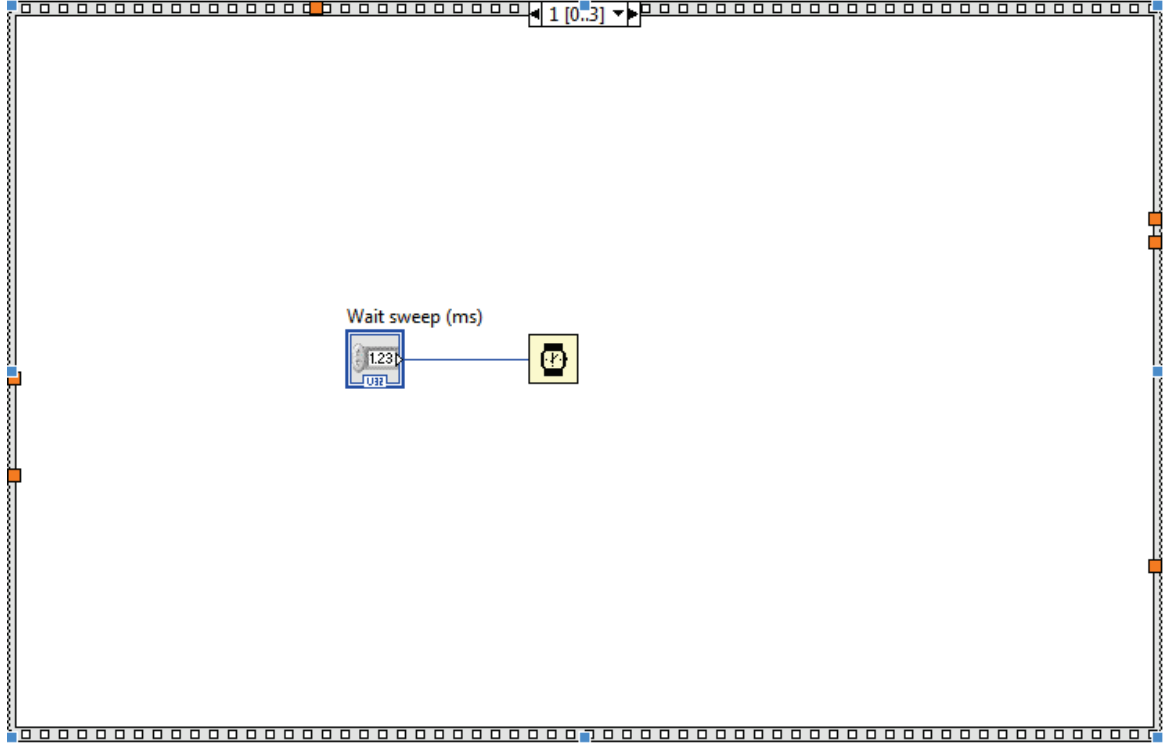

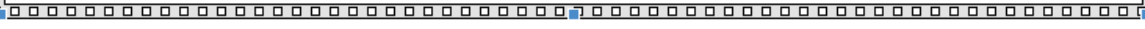

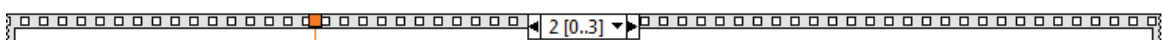

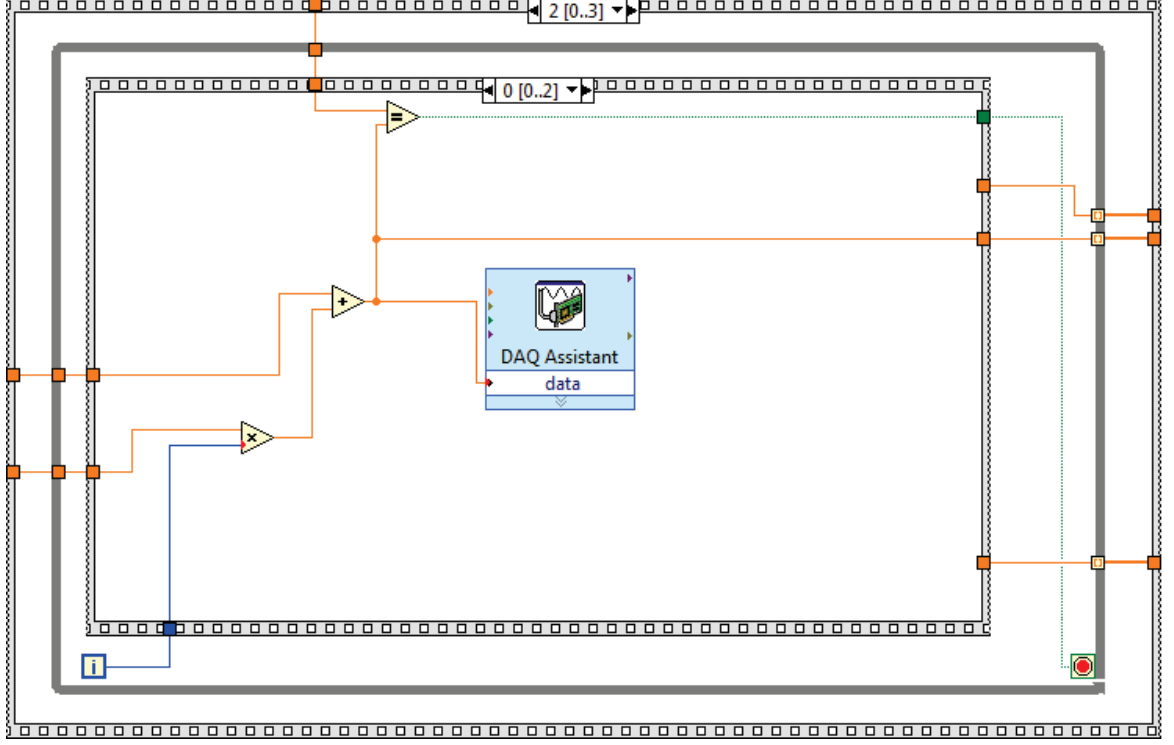


Appendix C. LabVIEW block diagrams of the developed signal processing and user interface software

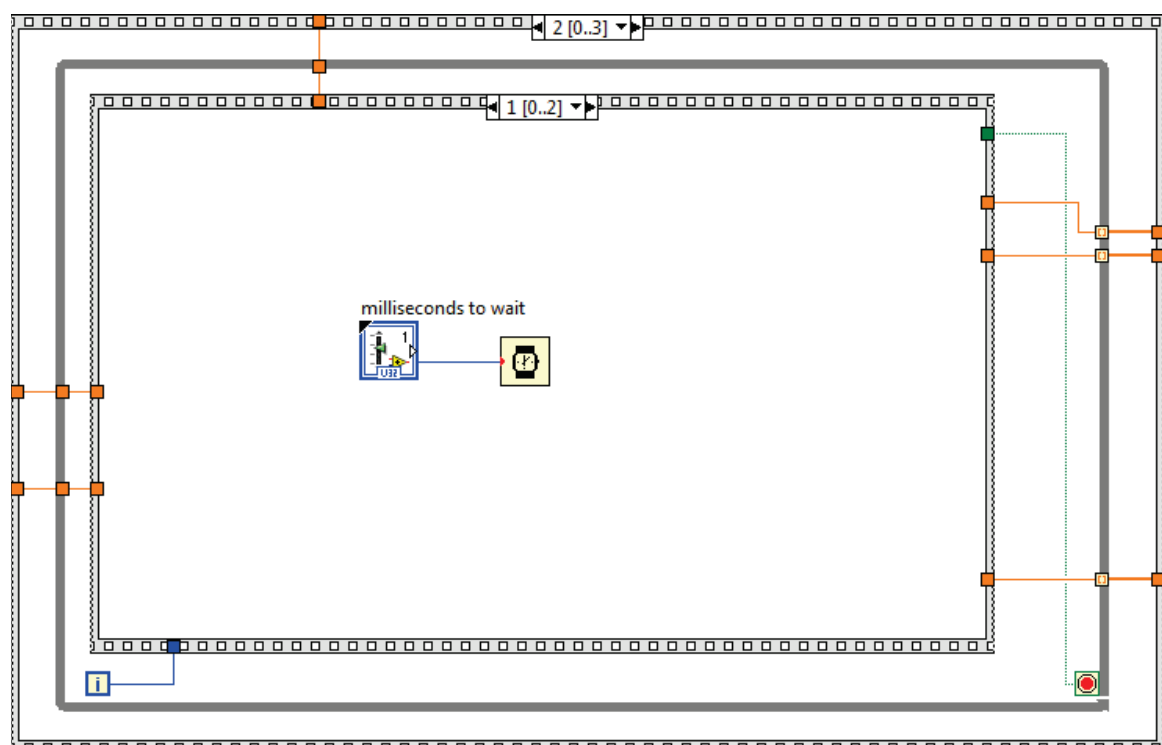

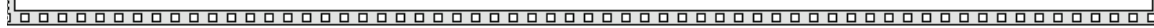

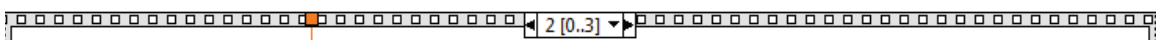

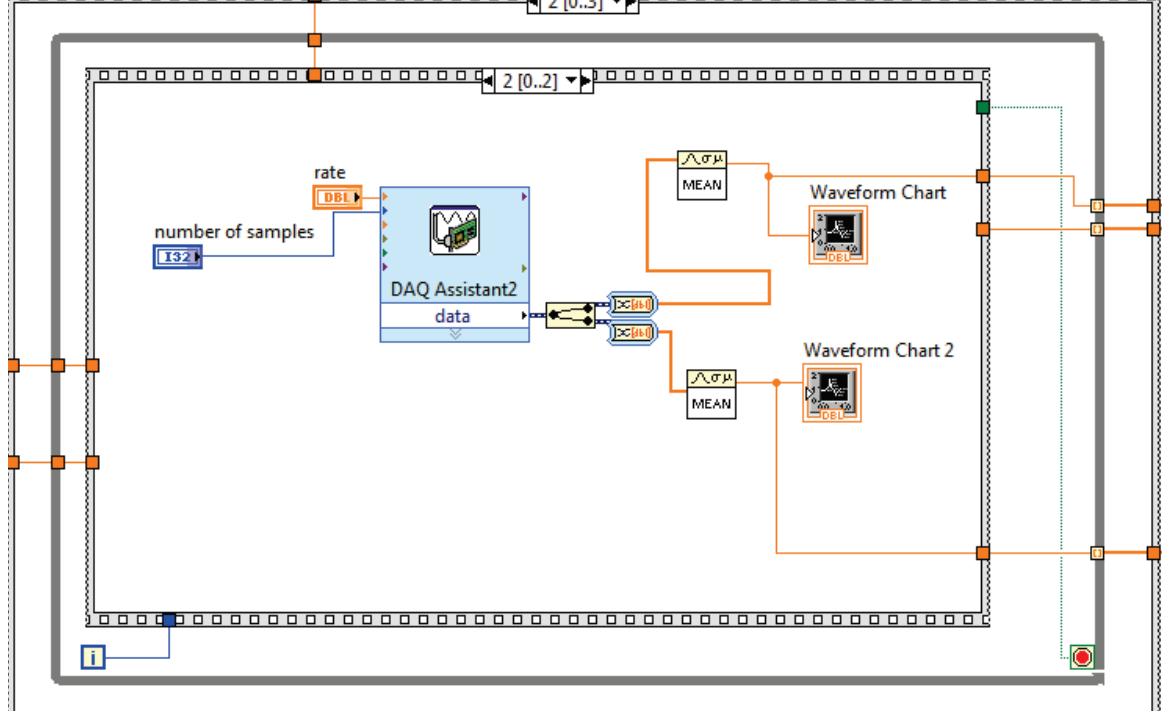

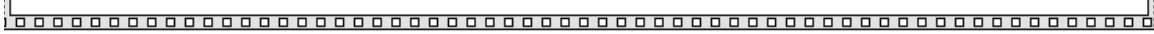

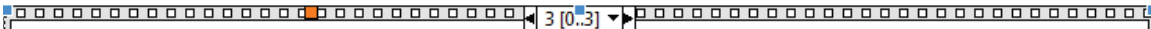

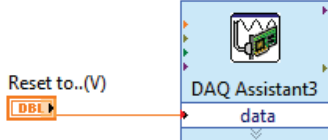




\section{Lock-in detection experimental pa- rameters}

\begin{tabular}{|l|c|c|c|}
\hline Parameter & Low Flow & High Flow & Units \\
\hline Analyte & Acetone & Acetone & - \\
\hline Analyte flow & $2.5 \times 10^{15}$ & $2.5 \times 10^{15}$ & Molecules/s \\
\hline Analyte Concentration & 2 & 0.65 & $\mathrm{ppm}$ \\
\hline Carrier gas & Nitrogen & Nitrogen & - \\
\hline Carrier gas flow & $3.05+/-0.05$ & $9.5+/-0.1$ & $1 / \mathrm{min}$ \\
\hline Pressure & 1 & 1 & $\mathrm{~atm}$. \\
\hline Temperature & 23 & 23 & ${ }^{\circ} \mathrm{C}$ \\
\hline Relative humidity & 5 & 5 & $\%$ \\
\hline Ion filter voltage & 700 & 700 & $\mathrm{~V}$ PP \\
\hline Ion filter duty cycle & 33 & 33 & $\%$ \\
\hline Ion filter frequency & 1 & 1 & $\mathrm{MHz}$ \\
\hline Sampling rate & 12345 & 12345 & $\mathrm{~Hz}$ \\
\hline Averaging time & 1 & 1 & $\mathrm{~s}$ \\
\hline Number of Samples & 12345 & 12345 & Samples/point \\
\hline Scan time & 105 & 105 & $\mathrm{~s}$ \\
\hline Modulation frequency & 1 to 812 & 1 to 812 & $\mathrm{~Hz}$ \\
\hline Modulation signal amplitude & 0.112 to 1.512 & 0.112 to 1.512 & $\mathrm{~V}_{\mathrm{RMS}}$ \\
\hline Low noise current amplifier gain & $1 \times 10^{10}-1 \times 10^{11}$ & $1 \times 10^{10}-1 \times 10^{11}$ & - \\
\hline Lock-in amplifier gain & 200 & 20 & - \\
\hline
\end{tabular}

Table D.1: Experimental parameters for the lock-in analysis. 



\section{Bibliography}

[1] Staff RAE Systems by Honeywell. The PID Handbook - Theory and Applications of DirectReading Photoionization Detectors (PIDs). RAE Systems Inc, USA, 3 edition, 2014.

[2] M. Reinhardt, G. Quiring, R. M. R. Wong, H. Wehrs, and J. Muller. Helium detection using a planar integrated micro-mass spectrometer. International Journal of Mass Spectrometry, 295(3):145-148, 2010.

[3] Hannelore Keller-Rudek; Geert K. Moortgat; Rolf Sander; Rüdiger Sörensen. The mpimainz uv vis spectral atlas of gaseous molecules of atmospheric interest.

[4] Arthur Mannering Tyndall. The Mobility of Positive Ions in Gases. The University Press, London, UK., 1 edition, 1938.

[5] R. A. Miller, E. G. Nazarov, G. A. Eiceman, and A. T. King. A mems radio-frequency ion mobility spectrometer for chemical vapor detection. Sensors and Actuators a-Physical, 91(3):301-312, 2001.

[6] E. V. Krylov, E. G. Nazarov, and R. A. Miller. Differential mobility spectrometer: Model of operation. International Journal of Mass Spectrometry, 266(1-3):76-85, 2007.

[7] H. W. Wang, C. L. Chen, Y. J. Liu, X. T. Zhang, D. Y. Kong, X. Z. Wang, and J. K. Luo. Humidity effects on resolution and sensitivity of uv-faims in vocs detection. Analytical Methods, 7(4):1401-1406, 2015.

[8] Texas Instruments. Lmc6001 ultra, ultra-low input current amplifier, 2015.

[9] M. Basanta, R. M. Jarvis, Y. Xu, G. Blackburn, R. Tal-Singer, A. Woodcock, D. Singh, R. Goodacre, C. L. P. Thomas, and S. J. Fowler. Non-invasive metabolomic analysis of breath using differential mobility spectrometry in patients with chronic obstructive pulmonary disease and healthy smokers. Analyst, 135(2):315-320, 2010.

[10] M. Schivo, W. Zhao, A. Aksenov, N. Kenyon, and C. Davis. Metabolomic analysis of exhaled breath condensates (ebc) in asthma and copd. Journal of Investigative Medicine, 59(1):205-205, 2011.

[11] Billy Boyle. Ion mobility mass spectrometry - the next 5 years, 2012. 
[12] H. Borsdorf and G. A. Eiceman. Ion mobility spectrometry: Principles and applications. Applied Spectroscopy Reviews, 41(4):323-375, 2006.

[13] H. Borsdorf, T. Mayer, M. Zarejousheghani, and G. A. Eiceman. Recent developments in ion mobility spectrometry. Applied Spectroscopy Reviews, 46(6):472-521, 2011.

[14] R. W. Purves, R. Guevremont, S. Day, C. W. Pipich, and M. S. Matyjaszczyk. Mass spectrometric characterization of a high-field asymmetric waveform ion mobility spectrometer. Review of Scientific Instruments, 69(12):4094-4105, 1998.

[15] R. Guevremont, R. W. Purves, D. A. Barnett, and L. Y. Ding. Ion trapping at atmospheric pressure (760 torr) and room temperature with a high-field asymmetric waveform ion mobility spectrometer. International Journal of Mass Spectrometry, 193(1):45-56, 1999.

[16] Edmond de Hoffmann and Vincent Stroobant. Mass Spectrometry: Principles and Applications. England, 3 edition, 2007.

[17] S. A. Trauger, E. P. Go, Z. X. Shen, J. V. Apon, B. J. Compton, E. S. P. Bouvier, M. G. Finn, and G. Siuzdak. High sensitivity and analyte capture with desorption/ionization mass spectrometry on silylated porous silicon. Analytical Chemistry, 76(15):4484-4489, 2004.

[18] J. A. Bradshaw, O. S. Ovchinnikova, K. A. Meyer, and D. E. Goeringer. Combined chemical and topographic imaging at atmospheric pressure via microprobe laser desorption/ionization mass spectrometry-atomic force microscopy. Rapid Communications in Mass Spectrometry, 23(23):3781-3786, 2009.

[19] O. S. Ovchinnikova, M. P. Nikiforov, J. A. Bradshaw, S. Jesse, and G. J. Van Berkel. Combined atomic force microscope-based topographical imaging and nanometer-scale resolved proximal probe thermal desorption/electrospray ionization-mass spectrometry. Acs Nano, 5(7):5526-5531, 2011.

[20] O. S. Ovchinnikova, V. Kertesz, and G. J. Van Berkel. Combining transmission geometry laser ablation and a non-contact continuous flow surface sampling probe/electrospray emitter for mass spectrometry based chemical imaging. Rapid Communications in Mass Spectrometry, 25(24):3735-3740, 2011.

[21] Jörg Müller, Grigoriy Quiring, Maria Reinhardt-Szyba, RéguloMiguelRamírez Wong, and Henning Wehrs. A Planar Integrated Micro-mass Spectrometer, chapter 14, pages 423-465. Springer US, 2012.

[22] C. M. Tassetti, R. Mahieu, J. S. Danel, O. Peyssonneaux, F. Progent, J. P. Polizzi, X. Machuron-Mandard, and L. Duraffourg. A mems electron impact ion source integrated in a microtime-of-flight mass spectrometer. Sensors and Actuators B-Chemical, 189:173-178, 2013.

[23] B. M. Kolakowski and Z. Mester. Review of applications of high-field asymmetric waveform ion mobility spectrometry (faims) and differential mobility spectrometry (dms). Analyst, 132(9):842-864, 2007. 
[24] R. Cumeras, E. Figueras, C. E. Davis, J. I. Baumbach, and I. Gracia. Review on ion mobility spectrometry. part 1: current instrumentation. Analyst, 140(5):1376-1390, 2015.

[25] R. Cumeras, E. Figueras, C. E. Davis, J. I. Baumbach, and I. Gracia. Review on ion mobility spectrometry. part 2: hyphenated methods and effects of experimental parameters. Analyst, 140(5):1391-1410, 2015.

[26] E. Krylov, E. G. Nazarov, R. A. Miller, B. Tadjikov, and G. A. Eiceman. Field dependence of mobilities for gas-phase-protonated monomers and proton-bound dimers of ketones by planar field asymmetric waveform ion mobility spectrometer (pfaims). Journal of Physical Chemistry A, 106(22):5437-5444, 2002.

[27] Ashley Wilks, Matthew Hart, Andrew Koehl, John Somerville, Billy Boyle, and David Ruiz-Alonso. Characterization of a miniature, ultra-high-field, ion mobility spectrometer. International Journal for Ion Mobility Spectrometry, 15(3):199-222, 2012.

[28] E. V. Krylov. Comparison of the planar and coaxial field asymmetrical waveform ion mobility spectrometer (faims). International Journal of Mass Spectrometry, 225(1):39-51, 2003.

[29] A. A. Shvartsburg, F. M. Li, K. Q. Tang, and R. D. Smith. High-resolution field asymmetric waveform ion mobility spectrometry using new planar geometry analyzers. Analytical Chemistry, 78(11):3706-3714, 2006.

[30] Russell Parris and Billy Bolye. Feasibility study of using faims to detect carbonyl sulfide in propane, 2013.

[31] C. P. Ireland and C. Ducati. Investigating the photo-oxidation of model indoor air pollutants using field asymmetric ion mobility spectrometry. Journal of Photochemistry and Photobiology a-Chemistry, 312:1-7, 2015.

[32] Zhuang Li, Bing-Tao Lin, De-Yi Kong, Chi-Lai Chen, Yu-Peng Cheng, Huan-Qin Wang, and Tao Mei. Faims of trace volatile organic compounds. Guang pu xue yu guang pu fen $x i=$ Guang $p u, 31(1): 12-5,2011$.

[33] Owlstone staff. Lonestar faims and lung cancer.

[34] B. Ells, D. A. Barnett, R. W. Purves, and R. Guevremont. Detection of nine chlorinated and brominated haloacetic acids at part-per-trillion levels using esi-faims-ms. Analytical Chemistry, 72(19):4555-4559, 2000.

[35] G. A. Eiceman, E. V. Krylov, E. G. Nazarov, and R. A. Miller. Separation of ions from explosives in differential mobility spectrometry by vapor-modified drift gas. Analytical Chemistry, 76(17):4937-4944, 2004.

[36] M. J. Manard, R. Trainham, S. Weeks, S. L. Coy, E. V. Krylov, and E. G. Nazarov. Differential mobility spectrometry/mass spectrometry: The design of a new mass spectrometer for 
real-time chemical analysis in the field. International Journal of Mass Spectrometry, 295(3):138-144, 2010.

[37] David R. Lide. CRC Handbook of Chemistry and Physics, volume 126 of Journal of the American Chemical Society. American Chemical Society, Boca Raton. USA, 84th edition, 2004.

[38] D. B. Robb and M. W. Blades. Factors affecting primary ionization in dopant-assisted atmospheric pressure photoionization (da-appi) for $\mathrm{lc} / \mathrm{ms}$. Journal of the American Society for Mass Spectrometry, 17(2):130-138, 2006.

[39] T. J. Kauppila, A. P. Bruins, and R. Kostiainen. Effect of the solvent flow rate on the ionization efficiency in atmospheric pressure photoionization-mass spectrometry. Journal of the American Society for Mass Spectrometry, 16(8):1399-1407, 2005.

[40] G.A. Eiceman and Z. Karpas. Ion Mobility Spectrometry, Second Edition. CRC Press, 2005.

[41] Alexandre Shvartsburg. DIFFERENTIAL ION MOBILITY SPECTROMETRY. CRC Press, Florida, USA, 2009.

[42] A. Heptner, P. Cochems, J. Langejuergen, F. Gunzer, and S. Zimmermann. Investigation of ion ion recombination at atmospheric pressure with a pulsed electron gun. Analyst, 137(21):5105-5112, 2012.

[43] J. Glosik and R. Plasil. The recombination rate coefficient of a protonated acetone dimer with electrons: indication of a temperature dependence. Journal of Physics B-Atomic Molecular and Optical Physics, 33(20):4483-4493, 2000.

[44] Manfred A. Biondi and Sanborn C. Brown. Measurement of electron-ion recombination. Phys. Rev., 76:1697-1700, Dec 1949.

[45] H. Jungblut, D. Hansen, and W. F. Schmidt. Ion-ion recombination in electronegative gases. Ieee Transactions on Electrical Insulation, 24(2):343-348, 1989. ISI Document Delivery No.: U7202 Times Cited: 6 Cited Reference Count: 14 Jungblut, h hansen, d schmidt, wf Ieee-inst electrical electronics engineers inc New york.

[46] A. A. Shvartsburg, R. D. Smith, A. Wilks, A. Koehl, D. Ruiz-Alonso, and B. Boyle. Ultrafast differential ion mobility spectrometry at extreme electric fields in multichannel microchips. Analytical Chemistry, 81(15):6489-6495, 2009.

[47] R. Guevremont. High-field asymmetric waveform ion mobility spectrometry: A new tool for mass spectrometry. Journal of Chromatography A, 1058(1-2):3-19, 2004.

[48] Stanford Research Systems. About lock-in amplifiers, application note 3.

[49] PerkinElmer Instruments. What is a lock-in amplifier?, 2000.

[50] Xiaohao Wang Fei Tang and Chulong Xu. FAIMS Biochemical Sensor Based on MEMS Technology. 2011. 


\section{Acknowledgements}

Many people deserve a mention for the help they gave me during these 4 years, but firstly, I would like to express my deepest gratitude to my parents for everything...

Also, I wish to express my sincere thanks to my advisors, Prof. Jürgen Brugger and Dr. Giovanni Boero, for giving me the opportunity to being involved in this project and for all their guidance during these last four years.

Beside my advisors, I would like to thank Prof. Kong and the members of his laboratory for hosting me during my visit to the Chinese Academy of Science, Hefei, China, and helping me to understand the complex, but extremely interesting, field of Ion Mobility Spectrometry. I would also like to express my gratitude to the Sino-Swiss Science and Technology Cooperation (SSSTC) program for the partial funding of this project.

Likewise, I want to thank Mr. Philippe Marmillod and Alessandro Matheoud for sharing with me some of their valuable knowledge in the electronics field, and Mrs. Marie Halm and Prof. Alexandre Schmid for the moral support and their valuable advice.

I thank my colleagues and friends in the Advanced NEMS group (GR-LVT) for the stimulating discussions, for the comforting talks, for all the beers and food we shared, for all the Game of Thrones episodes we watched together and for all the fun we have had in the last four years. Special thanks to Kaitlin Howell for spending her spare time reading this thesis and correcting my grammar and Annalisa De Pastina for translating it to Italian. I also thank my colleagues in LMIS1 for the good and the bad, because through both I learned.

Last, but not the least, I thank my lovely girlfriend for all her love and happy moments and my family for supporting me spiritually throughout writing this thesis and my life in general. 



\section{Curriculum Vitae}

\begin{tabular}{llll}
\hline Name: & Mario Andrés & Nationality: & Colombian \\
Surname: & Chavarría Varón & Residence permit: & B \\
Birth date/place: & May 19th 1985/Cali, CO. & Status: & Single \\
& & & \\
\hline
\end{tabular}

\section{ACADEMIC INFORMATION}

2012 - 2016 Ph.D. in Microsystems and Microelectronics Lausanne, $\mathbf{C H}$ École polytechnique fédérale de Lausanne

2009 - 2012 M.Sc. in Microelectronics and Microsystems Hamburg, DE TU Hamburg-Harburg

2003 - 2008 B.Sc. in Mechatronics Engineering

Cali, CO Universidad Autónoma de Occidente

\section{WORK EXPERIENCE}

$\begin{aligned} 07.2012-07.2016 & \text { EPFL } \\ & \text { Doctoral assistant } \\ & \text { Topic: Design and fabrication of a high sensitivity lon Mobility } \\ & \text { Spectrometer (FAIMS). } \\ & \text { Tasks: Design of high power-high frequency circuits for ion filtering, } \\ & \text { Weak current amplifiers design, PCB design, Electronic test and } \\ & \text { measurement, FAIMS characterization, Development of FAIMS } \\ & \text { simulation software, 3D printing, MEMS design and fabrication, } \\ & \text { implementation of soft ionization systems. }\end{aligned}$

04.2012 - 06.2012 TU Hamburg - Harburg Hamburg, DE Research Engineer at the Institute of Micro Systems Technology Tasks: Process analysis for the development of MEMS projects, electronic evaluation of isolation and semiconductor materials.

10.2009 - 03.2012 TU Hamburg - Harburg

Hamburg, DE Research Assistant at the Institute of Micro Systems Technology Tasks: Microfabrication of MEMS and NEMS for Mass Spectrometry, sensors and optic applications (photolithography, wet and dry etching, thin layer deposition processes, etc.).

09.2008 - 09.2009 Universidad Autónoma de Occidente

Researcher of the Advanced Materials for Micro and Nanotechnology Group

Tasks: Electronic characterization of materials for MEMS and NEMS applications. Development of software tools for the electronic characterization. 


\section{TECHNICAL SKILLS}

- Microfabrication:

- Thin layer deposition

- Dry and wet etching

- Photolithography

- Electron microscopy

- Atomic force Microscopy (AFM)

- Circuit Design

- Soft ionization

- 3D printing

- PCB prototyping

- Microcontroller programming

- PLC programming (Ladder logic)

- Programmable industrial robots

- Metrology

- CNC Machining

- Mass spectrometry

- Ion mobility spectrometry

- Software development

\section{SOFTWARE SKILLS}

- MATLAB

- Comsol multiphysics

- Altium Designer

- OriginPro

- Solid Edge

- Solid Works
- C, C++

- Ladder

- CleWin

- Mathematica

- FireWorks

- AutoCAD

\section{LANGUAGE SKILLS}

Spanish Native speaker

English Advanced

French Intermediate (B2)

\section{PUBLICATIONS \& PATENTS}

A complete list of publications, conferences and patents is included in the appendix section. 
APPENDIX: PUBLICATIONS \& PATENTS

\section{PAPERS}

02.2016 Mario Chavarría. Faruk Fonthal. "Electrical Investigation of Porous Silicon/p-Si Heterojunction Prepared by Electrochemical Etching". ECS Journal of Solid State Science and Technology, 4. February 2016. Vol. 5, pp. 3172-3175.

12.2015 Zhang Xiaotian, Chen Chilai, Liu Youjiang, Wang Hongwei, Zhang Lehua, Kong Deyi, Mario Chavarria. "Effects of the Discharge Parameters on the Efficiency and Stability of Ambient Metastable-Induced Desorption Ionization". Plasma Science and Technology, 17. December 2015. No. 12, pp.1048.

08.2013 Youjiang Liu, Lehua Zhang, Hongwei Wang, Xiaotian Zhang, Chilai Chen, Huanqin Wang, Deyi Kong, Mario A Chavarria, Juergen Brugger. "Identification of Toxic VOC Pollutants Using FAIMS". In proceeding of GreenCom-iThingsCPSCom. August 2013. 311, pp. 1698 - 1701.

08.2013 Lehua Zhang, Youjiang Liu, Hongwei Wang, Chilai Chen, Huanqin Wang, Deyi Kong, Xiaotian Zhang, Mario Chavarria, Juergen Brugger. "Detection of benzene series by two-dimensional FAIMS technique". In proceeding of GreenCom-iThings-CPSCom. August 2013. 311, pp. 1702-1705.

03.2013 K. Kaiser, M. Chavarria, S. Rajaretnam, T. Zehlicke, and J. Müller. "Pulling Of Highly Flexible Optical Fiber Bundles Using UV-Light Curing". In Proceedings of the International Conference on Microtechnologies in Medicine and Biology. Marina del Rey, California, USA. April 10 - 12, 2013. pp. 194-195.

06.2011 Faruk Fonthal and Mario A. Chavarria. "Impedance spectra under forward and reverse bias conditions in gold/porous silicon/p-Si structures". Phys. Status Solidi C 8. June 2011. No. 6, pp. $1913-1917$.

09.2009 M.A. Chavarria, F. Fonthal, "AC Eelectrical characterization of Au/porous silicon/p-Si thin films". In Proceedings of the IEEE Computer Society, 6th Int. Conf. CERMA 2009. Cuernavaca, Mexico. September 22 - 25, 2009. pp. 287 292.

09.2009 Mario Chavarría. Faruk Fonthal. "Electrical characterization of porous silicon on p-Si heterojunction". In Proceedings of the 11th International Conference on Advanced Materials (ICAM2009). Río de Janeiro, Brazil. September 20 - 25, 2009. pp. 137.

06.2009 M. Chavarria, F. Fonthal. "Electrical characterization and dielectric relaxation of Au/porous silicon contacts". Ceramic Transactions (CTs) book. Advances in Electroceramic Materials: Ceramic Transactions. Wiley InterScience. June 2009. USA. Vol. 204, pp. 113-118

\section{CONFERENCES}

03.2010 Materials for Advanced Metallization (MAM 2010)

F. Fonthal, E. S. Oliveros and M. Chavarria. "Temperature-dependent electrical transport of Au/porous silicon thin films heterojunction". Mechelen, Belgium. March 7-10, 2010.

09.2009 Electronics, Robotics and Automotive Mechanics Conference - IEEE (CERMA 2009)

M. Chavarria, F. Fonthal, "AC Electrical Characterization of Au/Porous Silicon/p-Si Thin Films”. Cuernavaca, Mexico. September 22 - 25, 2009.

$09.200911^{\text {th }}$ Int. Conference on Advanced Materials (ICAM 2009)

M. Chavarria, F. Fonthal, "Electrical Characterization of Porous Silicon on p-Si Heterojunction". Rio de Janeiro, Brazil. September 20 - 25, 2009.

$10.2008 \mathbf{2 1 4}^{\mathrm{IH}}$ Meeting of Electrochemical Society, Pacific Rim Meeting (PRIME 2008).

F. Fonthal, M. Chavarria, A. Rodriguez. "Electrical Transport And Dielectric Impedance Of Au/Porous Silicon Thin Films Structures". Honolulu, USA. October $12-17,2008$.

\section{PATENTS}

01.2010 F. Fonthal, M. Chavarria. "CEALAB (Characterization And Electrical Analysis Laboratory)". Colombia. National Copyrights Bureau. Book 13, Vol. 24 - 408. 